\title{
Catalyst- and Substituent-Controlled Regio- and Stereoselective Synthesis of Indolyl Acrylates by Lewis-Acid- Catalyzed Direct Functionalization of 3-Formylindoles with Diazo Esters
}

\author{
Sana Jamshaid, Shreedhar Devkota, and Yong Rok Lee* \\ School of Chemical Engineering, Yeungnam University, Gyeongsan 38541, Republic of Korea. \\ E-mail: yrlee@ yu.ac.kr; Fax: +82-53-810-4631; Tel: +82-53-810-2529
}

Supplementary Information 


\section{TABLE OF CONTENTS}

$\begin{array}{ll}\text { General remarks } & \text { S3 }\end{array}$

General procedure for the synthesis of 3, 4, and $6 \quad 53$

$\begin{array}{ll}\text { General procedure for the synthesis of compounds } 7 & \text { S4 }\end{array}$

Representative 1 mmol scale reaction for the synthesis of 3a

Representative $1 \mathrm{mmol}$ scale reaction for the synthesis of $7 \mathrm{a} \quad \mathrm{S4}$

$\begin{array}{ll}\text { Additional experiments: } & \text { S5 }\end{array}$

i) General procedure for the formation of $2 a^{\prime} \quad$ S5

ii) Deuterium-labeling experiments $\quad$ S5

iii) General procedure for the reaction of benzaldehyde with 2a S5

iv) General procedure for the synthesis of 20 and $21 \quad$ S6

Characterization data of starting compounds $\quad$ S6

$\begin{array}{ll}\text { Characterization data of synthesized products } & \text { S7 }\end{array}$

${ }^{1} \mathrm{H}$ NMR and ${ }^{13} \mathrm{C}$ NMR spectra of starting compounds $\quad$ S25

${ }^{1} \mathrm{H}$ NMR and ${ }^{13} \mathrm{C}$ NMR spectra of synthesized products $\quad$ S28

$\begin{array}{ll}\text { References } & \text { S77 }\end{array}$

Method of crystal growth for compounds $4 \mathrm{~m}, 6 e$ and $7 \mathrm{c}$ and 578 instrumentation

$\begin{array}{ll}\text { X-ray structure and data of compound 4m, 6e, and 7c } & \text { S79 }\end{array}$ 


\section{General remarks}

All experiments were carried out in open-air unless stated otherwise. Merck precoated silica gel plates (Art. 5554) treated with a fluorescent indicator were used for analytical thin-layer chromatography (TLC). Column chromatography was performed using silica gel 9385 (Merck). Melting points are uncorrected and were determined using Fisher-Johns Melting Point Apparatus. ${ }^{1} \mathrm{H}$ NMR and ${ }^{13} \mathrm{C}$ NMR spectra were recorded on VNS (600 and $150 \mathrm{MHz}$ or 300 and $75 \mathrm{MHz}$ ) spectrometers at the core research support center for natural products and medical materials of Yeungnam University. The NMR spectra recorded in $\mathrm{CDCl}_{3}$ using $\delta=7.24$ and $77.00 \mathrm{ppm}$ as the residual solvent chemical shifts. All chemical shifts $(\delta)$ are expressed in units of ppm and $J$ values are given in Hz. Multiplicities are abbreviated as follows: $\mathrm{s}=$ singlet, $\mathrm{d}=$ doublet, $\mathrm{t}=$ triplet, $\mathrm{q}=$ quartet, $\mathrm{m}=$ multiplet or overlap of nonequivalent resonances, and $\mathrm{dd}=$ doublet of doublets. Infrared (IR) spectra were recorded on a PerkinElmer Spectrum Two ${ }^{\mathrm{TM}}$ IR spectrometer with frequencies expressed in $\mathrm{cm}^{-1}$, and high-resolution mass spectrometry (HRMS) was carried out using a JEOL JMS-700 spectrometer (Magnetic sector-Electric sector double focusing mass analyzer) at the Korea Basic Science Institute. The compounds 1h, 1q, 5c and 7aD were analysed for HRMS using Thermo Fisher Q exactive orbitrap mass spectrometer. The crystal structures were determined by single-crystal diffraction methods at the Korea Basic Science Institute (KBSI, Western Seoul Center, Korea).

Starting materials $\mathbf{1 a}$ and $\mathbf{1 k}$ are commercially available and were used directly as purchased from Sigma Aldrich. The starting 3-formylindoles 1b-1j, 1l-1x, and 5a-5e were prepared according to the literature procedures ${ }^{1-2}$ and all are known compounds. Surprisingly, following the same procedure for the synthesis of 5a, 5c was obtained as a minor product. The starting materials 1h, 1q and $\mathbf{5 c}$ are unknown compounds, therefore their experimental data including yields, melting points, characterization data ${ }^{1} \mathrm{H}$ and ${ }^{13} \mathrm{C}$ NMR spectras and HRMS data are provided. The starting diazoacetate 2a was purchased from Sigma Aldrich, while $\mathbf{2 b - 2 h}$ are known compounds and were prepared according to the reported method. ${ }^{3}$ For the deuteriumlabeling experiment, the deuterated 3-formyl indole 1aD was prepared according to reported method. ${ }^{4}$ 


\section{General procedure for the synthesis of 3,4 , and 6}

A mixture of 3-formyl indole $1(0.5 \mathrm{mmol})$, diazoacetate $2(1.1 \mathrm{mmol}, 2.2 \mathrm{eq})$ and $5 \mathrm{~mol} \%$ of $\operatorname{In}(\mathrm{OTf})_{3}$ in 1,2-DCE $(3 \mathrm{~mL})$ was stirred on an oil bath at reflux temperature for 5-6 $\mathrm{h}$. When the reaction was completed as indicated by TLC, the volatiles were removed in vacuo and the residue was purified by silica gel column chromatography (EtOAc/Hexanes 1/9) to obtain the desired products.

\section{General procedure for the synthesis of compounds 7}

A mixture of 3-formyl indole $\mathbf{1}(0.5 \mathrm{mmol})$, diazoacetate $\mathbf{2}(1.1 \mathrm{mmol}, 2.2 \mathrm{eq})$ in 1,2-DCE (3 mL) under $\mathrm{N}_{2}$ was stirred at room temperature. Then, $\mathrm{BF}_{3} \cdot \mathrm{OEt}_{2}(0.3 \mathrm{mmol}, 38 \mu \mathrm{L})$ was slowly added through the syringe and left the reaction mixture to stir at $\mathrm{rt}$. When the reaction was completed after $4 \mathrm{~h}$ as indicated by TLC, the volatiles were removed in vacuo and the residue was purified by silica gel column chromatography (EtOAc/Hexanes 1/9) to obtain the desired products.

\section{Representative $1 \mathrm{mmol}$ scale reaction for the synthesis of $3 \mathrm{a}$}

A mixture 1-methyl-1 $H$-indole-3-carbaldehyde (1a) $(159 \mathrm{mg}, 1.0 \mathrm{mmol}$,), ethyl 2-diazoacetate (2a) $\left(250 \mathrm{mg}, 2.2 \mathrm{mmol}, 2.2 \mathrm{eq}\right.$,) and $10 \mathrm{~mol} \%(56 \mathrm{mg})$ of $\operatorname{In}(\mathrm{OTf})_{3}$ in 1,2-DCE (3 mL) was stirred on an oil bath at reflux temperature for $6 \mathrm{~h}$. When the reaction was completed as indicated by TLC, the volatiles were removed in vacuo and the residue was purified by silica gel column chromatography (EtOAc/Hexanes 1/9) to obtain the desired product 3a $(186 \mathrm{mg})$ in $76 \%$ yield as a white solid.

\section{Representative $1 \mathrm{mmol}$ scale reaction for the synthesis of $7 \mathrm{a}$}

A mixture of 1-methyl-1H-indole-3-carbaldehyde (1a) $(159 \mathrm{mg}, 1.0 \mathrm{mmol})$, ethyl 2-diazoacetate 2a $(250 \mathrm{mg}, 2.2 \mathrm{mmol}, 2.2 \mathrm{eq})$ in 1,2-DCE ( $3 \mathrm{~mL})$ under $\mathrm{N}_{2}$ was stirred at room temperature. Then, $\mathrm{BF}_{3} \cdot \mathrm{OEt}_{2}(84 \mathrm{mg}, 0.6 \mathrm{mmol}, 76 \mu \mathrm{L}$ ) was slowly added through the syringe and left the reaction mixture to stir at rt. When the reaction was completed after $4 \mathrm{~h}$ as indicated by TLC, the volatiles were removed in vacuo and the residue was purified by silica gel column chromatography (EtOAc/Hexanes $1 / 9)$ to obtain product $\mathbf{7 a}(176 \mathrm{mg}$ ) in $72 \%$ yield as a pale yellow solid . 


\section{Additional experiments:}

\section{i) General procedure for the formation of $2 \mathbf{a}^{\prime}$}

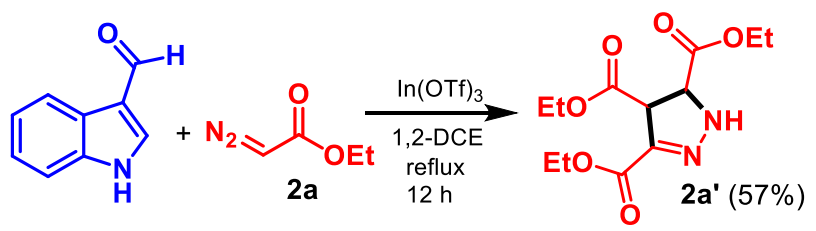

A mixture $1 H$-indole-3-carbaldehyde (145 mg, $1.0 \mathrm{mmol}$ ), ethyl 2-diazoacetate (2a) (250 mg, 2.2 mmol, $2.2 \mathrm{eq})$ and $10 \mathrm{~mol} \%(56 \mathrm{mg})$ of $\operatorname{In}(\mathrm{OTf})_{3}$ in 1,2-DCE (3 mL) was stirred on an oil bath at reflux temperature for $12 \mathrm{~h}$. When the reaction was completed as indicated by TLC, the volatiles were removed in vacuo and the residue was purified by silica gel column chromatography (EtOAc/Hexanes 3/7) as white crystalline product $\mathbf{2} \mathbf{a}^{\prime}$ in $\mathbf{5 7 \%}$ yield.

\section{ii) Deuterium-labeling experiments}

a. Reaction of $1 \mathrm{aD}$ with $2 \mathrm{a}$ under $\operatorname{In}(\mathrm{OTf})_{3}$ catalysis: The reaction of deuterated 1-methyl-1Hindole-3-carbaldehyde- $d$ 1aD $(0.5 \mathrm{mmol})$ with diazoacetate $\mathbf{2 a}(1.1 \mathrm{mmol}, 2.2 \mathrm{eq})$ in $5 \mathrm{~mol} \%$ of $\operatorname{In}(\mathrm{OTf})_{3}$ in 1,2-DCE $(3 \mathrm{~mL})$ was stirred on an oil bath at reflux temperature for $6 \mathrm{~h}$. After the reaction was completed as indicated by TLC, the volatiles were removed in vacuo and the residue was purified by silica gel column chromatography (EtOAc/Hexanes 1/9) to obtain the desired product 3aD.

b. Reaction of $1 \mathrm{aD}$ with $2 \mathrm{a}$ under $\mathbf{B F}_{3} \cdot \mathbf{O E t}_{2}$ catalysis: The reaction of deuterated 1-methyl$1 H$-indole-3-carbaldehyde- $d \mathbf{~ 1 a D}(0.5 \mathrm{mmol})$ with diazoacetate $\mathbf{2 a}(1.1 \mathrm{mmol}, 2.2 \mathrm{eq})$ in 1,2DCE $(3 \mathrm{~mL})$ under $\mathrm{N}_{2}$ was stirred at room temperature. Then, $\mathrm{BF}_{3} \cdot \mathrm{OEt}_{2}(0.3 \mathrm{mmol}, 38 \mu \mathrm{L})$ was slowly added through the syringe and left the reaction mixture to stir at rt. When the reaction was completed after $4 \mathrm{~h}$ as indicated by TLC, the volatiles were removed in vacuo and the residue was purified by silica gel column chromatography (EtOAc/Hexanes 1/9) to obtain the 7aD.

\section{iii) General procedure for the reaction of benzaldehyde with 2 a}

A mixture of benzaldehyde $(\mathbf{5 g})(0.5 \mathrm{mmol})$, with ethyl diazoacetate (2a) $(1.2 \mathrm{eq})$ in $5 \mathrm{~mol} \%$ of $\operatorname{In}(\mathrm{OTf})_{3}$ in 1,2-DCE $(3 \mathrm{~mL})$ was stirred on an oil bath at reflux temperature for $6 \mathrm{~h}$. After the 
reaction was completed as indicated by TLC, the volatiles were removed in vacuo and the residue was purified by silica gel column chromatography (EtOAc/Hexanes : 1/9) to obtain $\mathbf{A}$ and $\mathbf{B}(65 \%$, combined yield).

\section{iv) General procedure for the synthesis of 20 and 21}

A mixture of compound 3a $(0.25 \mathrm{mmol})$, with alkyl halides and $\mathrm{K}_{2} \mathrm{CO}_{3}(0.5 \mathrm{eq})$ were stirred in toluene $(2 \mathrm{~mL})$ at room temperature for $12 \mathrm{~h}$. When the reaction was complete as indicated by TLC, the volatiles were evaporated in vacuo and the residue was purified by silica gel column chromatography $($ EtOAc/Hexanes $=1 / 9)$ to obtain the desired products $\mathbf{2 0 - 2 1}$.

\section{Characterization data of starting compounds}

\section{1-(1-Phenylethyl)-1H-indole-3-carbaldehyde (1h)}

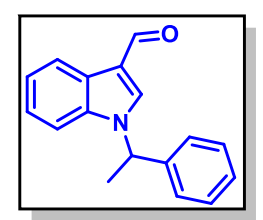

The title compound was prepared according to the general procedure. The reaction was carried out on $2 \mathrm{mmol}$ scale. The product was obtained as a yellow solid. Yield: 69\% (343 mg) ; mp 68-70 ${ }^{\circ} \mathrm{C} .{ }^{1} \mathrm{H}$ NMR (600 MHz, $\left.\mathrm{CDCl}_{3}\right) \delta 9.95$ (s, 1H), $8.29(\mathrm{~d}, J=7.8 \mathrm{~Hz}, 1 \mathrm{H}), 7.82(\mathrm{~s}, 1 \mathrm{H}), 7.25-7.13(\mathrm{~m}, 6 \mathrm{H}), 7.10-7.06(\mathrm{~m}$, $2 \mathrm{H}), 5.57(\mathrm{q}, J=7.2 \mathrm{~Hz}, 1 \mathrm{H}), 1.85(\mathrm{~d}, J=7.2 \mathrm{~Hz}, 3 \mathrm{H}) ;{ }^{13} \mathrm{C} \mathrm{NMR}\left(150 \mathrm{MHz}, \mathrm{CDCl}_{3}\right) \delta 184.6$, 140.6, 137.3, 136, 128.9, 128.1, 125.8, 125.8, 125.5, 123.9, 122.9, 121.9, 118.2, 110.9, 55.8, 21.5; HRMS ESI $m / z,\left[\mathrm{MH}^{+}\right]$calcd for $\mathrm{C}_{17} \mathrm{H}_{16} \mathrm{NO}: 250.1226$. Found: 250.1224.

\section{1-Methyl-5-(naphthalen-2-yl)-1H-indole-3-carbaldehyde (1q)}

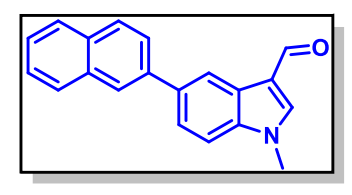

The title compound was prepared according to the literature procedure. ${ }^{2 \mathrm{c}}$ The reaction was carried out on $1 \mathrm{mmol}$ scale. The product was obtained as a pink solid. Yield: 86\% (245 mg) ; mp 195-197 ${ }^{\circ} \mathrm{C} .{ }^{1} \mathrm{H}$ NMR (600 MHz, $\left.\mathrm{CDCl}_{3}\right) \delta 9.97(\mathrm{~s}, 1 \mathrm{H}), 8.65(\mathrm{~s}, 1 \mathrm{H}), 8.11(\mathrm{~s}, 1 \mathrm{H}), 7.90(\mathrm{t}, J=8.4 \mathrm{~Hz}, 2 \mathrm{H}), 7.84(\mathrm{t}, J=9.6 \mathrm{~Hz}$, 2H), $7.72(\mathrm{dd}, J=1.2,8.4 \mathrm{~Hz}, 1 \mathrm{H}), 7.70(\mathrm{~s}, 1 \mathrm{H}), 7.51-7.44(\mathrm{~m}, 2 \mathrm{H}), 7.43(\mathrm{~d}, J=8.4 \mathrm{~Hz}, 1 \mathrm{H})$,

$3.88(\mathrm{~s}, 3 \mathrm{H}) ;{ }^{13} \mathrm{C} \mathrm{NMR}\left(150 \mathrm{MHz}, \mathrm{CDCl}_{3}\right) \delta 184.1,138.5,137.2,136.3,133.5,132.2,128.1$, 127.9, 127.3, 126.0, 125.8, 125.7, 125.6, 125.5, 123.8, 120.6, 117.9, 110.1, 33.6; HRMS ESI $m / z$ $\left[\mathrm{MH}^{+}\right]$calcd for $\mathrm{C}_{20} \mathrm{H}_{16} \mathrm{NO}: 286.1226$. Found: 286.1223. 


\section{2-Ethyl-1-methyl-1H-indole-3-carbaldehyde (5c)}

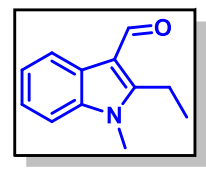

The title compound was prepared according to the general procedure. The reaction was carried out on $2 \mathrm{mmol}$ scale. The product was obtained as a yellow solid. Yield: $45 \%$ (168 mg) ; mp 74-76 C. ${ }^{1} \mathrm{H}$ NMR $\left(600 \mathrm{MHz}, \mathrm{CDCl}_{3}\right) \delta 10.10$ (s, 1H), 8.26-8.24 (m, 1H), 7.28-7.25 (m, 3H), 3.65 (s, 3H), 3.03 (q, $J=7.8 \mathrm{~Hz}, 2 \mathrm{H}), 1.29$ (t, $J=7.8$ $\mathrm{Hz}, 3 \mathrm{H}) ;{ }^{13} \mathrm{C} \mathrm{NMR}\left(150 \mathrm{MHz}, \mathrm{CDCl}_{3}\right) \delta 183.7,153.4,136.9,125.5,123.0,122.7,120.8,112.9$, 109.2, 29.4, 17.6, 14.5; HRMS ESI $m / z\left[\mathrm{MH}^{+}\right]$calcd for $\mathrm{C}_{12} \mathrm{H}_{14} \mathrm{NO}$ : 188.1070. Found: 188.1067.

\section{Characterization data of synthesized products}

\section{Ethyl (Z)-2-hydroxy-3-(1-methyl-1H-indol-3-yl)acrylate (3a)}

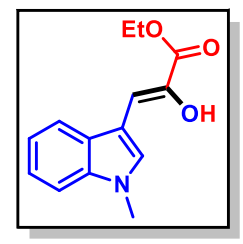

The title compound was prepared according to the general procedure. The product was obtained as a white solid. EtOAc:Hex (1:9); Yield: 76\% (93 mg); mp 96-98 ${ }^{\circ} \mathrm{C} .{ }^{1} \mathrm{H}$ NMR $\left(600 \mathrm{MHz}, \mathrm{CDCl}_{3}\right) \delta 7.83(\mathrm{~s}, 1 \mathrm{H}), 7.78(\mathrm{~d}, J=8.4 \mathrm{~Hz}$, $1 \mathrm{H}), 7.32(\mathrm{~d}, J=7.8 \mathrm{~Hz}, 1 \mathrm{H}), 7.27(\mathrm{t}, J=8.4 \mathrm{~Hz}, 1 \mathrm{H}), 7.20(\mathrm{t}, J=8.4 \mathrm{~Hz}, 1 \mathrm{H})$, $6.94(\mathrm{~s}, 1 \mathrm{H}), 6.25(\mathrm{~s}, 1 \mathrm{H}), 4.37(\mathrm{q}, J=7.2 \mathrm{~Hz}, 2 \mathrm{H}), 3.81(\mathrm{~s}, 3 \mathrm{H}), 1.41(\mathrm{t}, J=7.2 \mathrm{~Hz}, 3 \mathrm{H}) ;{ }^{13} \mathrm{C}$ NMR $\left(150 \mathrm{MHz}, \mathrm{CDCl}_{3}\right) \delta 165.9,136.3,136.1,131.7,127.3,122.1,119.8,118.5,109.2,108.9$, 103.7, 61.8, 32.9, 14.2; IR (ATR) 3402, 1658, 1391, 1325, 1231, 1118, 1016, 729, $508 \mathrm{~cm}^{-1}$; HRMS $m / z\left[\mathrm{M}^{+}\right]$calcd for $\mathrm{C}_{14} \mathrm{H}_{15} \mathrm{NO}_{3}: 245.1052$. Found: 245.1053 .

\section{Isopropyl (Z)-2-hydroxy-3-(1-methyl-1H-indol-3-yl)acrylate (3b)}

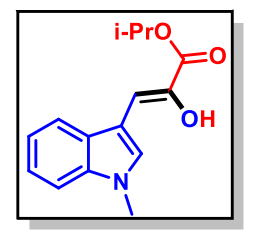

The title compound was prepared according to the general procedure. The product was obtained as a pink solid. EtOAc:Hex (1:9); Yield: 71\% (92 mg); mp 144-146 C. ${ }^{1} \mathrm{H}$ NMR $\left(600 \mathrm{MHz}, \mathrm{CDCl}_{3}\right) \delta 7.82(\mathrm{~s}, 1 \mathrm{H}), 7.78(\mathrm{~d}, J=7.8 \mathrm{~Hz}, 1 \mathrm{H})$, $7.32(\mathrm{~d}, J=7.2 \mathrm{~Hz}, 1 \mathrm{H}), 7.26(\mathrm{t}, J=8.4 \mathrm{~Hz}, 1 \mathrm{H}), 7.19$ (t, $J=7.2 \mathrm{~Hz}, 1 \mathrm{H}), 6.90(\mathrm{~s}, 1 \mathrm{H}), 6.26$ (s, $1 \mathrm{H}), 5.19(\mathrm{p}, J=6.6 \mathrm{~Hz}, 1 \mathrm{H}), 3.82(\mathrm{~s}, 3 \mathrm{H}), 1.37(\mathrm{~d}, J=6.0 \mathrm{~Hz}, 6 \mathrm{H}) ;{ }^{13} \mathrm{C} \mathrm{NMR}\left(150 \mathrm{MHz}, \mathrm{CDCl}_{3}\right)$ $\delta 165.4,136.4,136.3,131.6,127.2,121.9,119.7,118.5,109.2$, 108.9, 103.2, 69.6, 32.9, 21.7; IR (ATR) 3392, 2975, 1663, 1462, 1367, 1246, 1091, 741, 649, $417 \mathrm{~cm}^{-1}$; HRMS m/z [M+ $\mathrm{M}^{+}$calcd for $\mathrm{C}_{15} \mathrm{H}_{17} \mathrm{NO}_{3}$ : 259.1208. Found: 259.1210. 


\section{Butyl (Z)-2-hydroxy-3-(1-methyl-1H-indol-3-yl)acrylate (3c)}

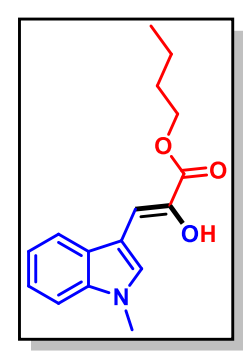

The title compound was prepared according to the general procedure. The product was obtained as a offwhite solid. EtOAc:Hex (1:9); Yield: 63\% (86 mg); mp 164-166 C. ${ }^{1} \mathrm{H}$ NMR $\left(600 \mathrm{MHz}, \mathrm{CDCl}_{3}\right) \delta 7.83(\mathrm{~s}, 1 \mathrm{H}), 7.77(\mathrm{~d}, J=7.8 \mathrm{~Hz}$, 1H), 7.32 (d, $J=8.4 \mathrm{~Hz}, 1 \mathrm{H}), 7.27$ (t, $J=7.8 \mathrm{~Hz}, 1 \mathrm{H}), 7.19$ (t, $J=7.2 \mathrm{~Hz}, 1 \mathrm{H})$, $6.93(\mathrm{~s}, 1 \mathrm{H}), 6.24(\mathrm{~s}, 1 \mathrm{H}), 4.31(\mathrm{t}, J=6.6 \mathrm{~Hz}, 2 \mathrm{H}), 3.82(\mathrm{~s}, 3 \mathrm{H}), 1.76(\mathrm{p}, J=7.2$ $\mathrm{Hz}, 2 \mathrm{H}), 1.48(\mathrm{q}, J=7.2 \mathrm{~Hz}, 2 \mathrm{H}), 1.00(\mathrm{t}, J=7.8 \mathrm{~Hz}, 3 \mathrm{H}) ;{ }^{13} \mathrm{C}$ NMR $(150 \mathrm{MHz}$ $\left.\mathrm{CDCl}_{3}\right) \delta 165.9,136.3,136.0,131.7,127.2,122.0,119.8,118.4,109.2,108.9,103.5,65.6,32.9$, 30.5, 18.9, 13.5; IR (ATR) 3402, 2955, 1729, 1463, 1219, 1062, 928, 728, $428 \mathrm{~cm}^{-1}$; HRMS m/z $\left[\mathrm{M}^{+}\right]$calcd for $\mathrm{C}_{16} \mathrm{H}_{19} \mathrm{NO}_{3}: 273.1365$. Found: 273.1367 .

\section{Allyl (Z)-2-hydroxy-3-(1-methyl-1H-indol-3-yl)acrylate (3d)}

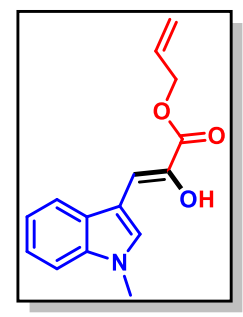

The title compound was prepared according to the general procedure. The product was obtained as a light brown solid. EtOAc:Hex (1:9); Yield: 73\% (94 $\mathrm{mg}) ; \mathrm{mp}$ 60-62 ${ }^{\circ} \mathrm{C} .{ }^{1} \mathrm{H} \mathrm{NMR}\left(600 \mathrm{MHz}, \mathrm{CDCl}_{3}\right) \delta 7.84$ (s, 1H), 7.77 (d, $J=7.8$ $\mathrm{Hz}, 1 \mathrm{H}), 7.32(\mathrm{~d}, J=8.4 \mathrm{~Hz}, 1 \mathrm{H}), 7.26(\mathrm{t}, J=7.8 \mathrm{~Hz}, 1 \mathrm{H}), 7.19$ (t, $J=7.8 \mathrm{~Hz}$, $1 \mathrm{H}), 6.98(\mathrm{~s}, 1 \mathrm{H}), 6.19(\mathrm{~s}, 1 \mathrm{H}), 6.05-5.94(\mathrm{~m}, 1 \mathrm{H}), 5.43(\mathrm{dd}, J=1.8,17.4 \mathrm{~Hz}$, $1 \mathrm{H}), 5.32(\mathrm{dd}, J=1.2,10.2 \mathrm{~Hz}, 1 \mathrm{H}), 4.80(\mathrm{~d}, J=6.0 \mathrm{~Hz}, 2 \mathrm{H}), 3.82(\mathrm{~s}, 3 \mathrm{H}) ;{ }^{13} \mathrm{C} \mathrm{NMR}(150 \mathrm{MHz}$, $\left.\mathrm{CDCl}_{3}\right) \delta 165.6,136.3,135.7,131.8,131.6,127.2,122.1,119.8,118.5,118.4,109.2,108.8$, 104.2, 66.2, 32.9; IR (ATR) 3408, 2929, 1724, 1460, 1221, 982, 740, $562 \mathrm{~cm}^{-1}$; HRMS m/z [M+] calcd for $\mathrm{C}_{15} \mathrm{H}_{15} \mathrm{NO}_{3}$ : 257.1052. Found: 257.1052.

\section{Benzyl (Z)-2-hydroxy-3-(1-methyl-1H-indol-3-yl)acrylate (3e)}

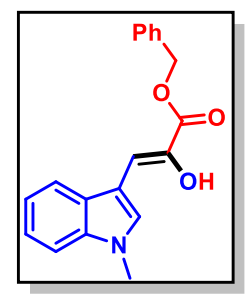

The title compound was prepared according to the general procedure. The product was obtained as a light pink solid. EtOAc:Hex (1:9); Yield: 87\% (134 $\mathrm{mg}) ; \mathrm{mp} 96-98{ }^{\circ} \mathrm{C} .{ }^{1} \mathrm{H} \mathrm{NMR}\left(600 \mathrm{MHz}, \mathrm{CDCl}_{3}\right) \delta 7.83(\mathrm{~s}, 1 \mathrm{H}), 7.74(\mathrm{~d}, J=7.9$ $\mathrm{Hz}, 1 \mathrm{H}), 7.44$ (d, $J=7.2 \mathrm{~Hz}, 2 \mathrm{H}), 7.40$ (t, $J=8.4 \mathrm{~Hz}, 2 \mathrm{H}), 7.36(\mathrm{~d}, J=7.2 \mathrm{~Hz}$, $1 \mathrm{H}), 7.31(\mathrm{~d}, J=7.8 \mathrm{~Hz}, 1 \mathrm{H}), 7.26(\mathrm{~d}, J=7.2 \mathrm{~Hz}, 1 \mathrm{H}), 7.18(\mathrm{t}, J=6.6 \mathrm{~Hz}, 1 \mathrm{H})$, $6.99(\mathrm{~s}, 1 \mathrm{H}), 6.19(\mathrm{~s}, 1 \mathrm{H}), 5.34(\mathrm{~s}, 2 \mathrm{H}), 3.82(\mathrm{~s}, 3 \mathrm{H}) ;{ }^{13} \mathrm{C} \mathrm{NMR}\left(150 \mathrm{MHz}, \mathrm{CDCl}_{3}\right) \delta 165.9,136.5$, 135.9, 135.6, 132.1, 128.6, 128.4, 128.2, 127.4, 122.2, 120.0, 118.7, 109.4, 109.1, 104.5, 67.5, 
33.1; IR (ATR) 3452, 2951, 1707, 1362, 1162, $780 \mathrm{~cm}^{-1}$; HRMS m/z [M+c calcd for $\mathrm{C}_{19} \mathrm{H}_{17} \mathrm{NO}_{3}$ : 307.1208. Found: 307.1210.

Phenethyl (Z)-2-hydroxy-3-(1-methyl-1H-indol-3-yl)acrylate (3f)

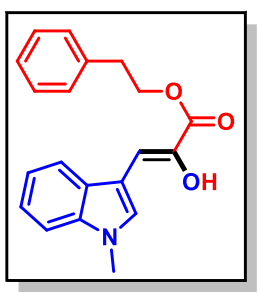

The title compound was prepared according to the general procedure. The product was obtained as a light brown solid. EtOAc:Hex (1:9); Yield: 68\% (109 mg); mp 133-135 ${ }^{\circ} \mathrm{C} .{ }^{1} \mathrm{H}$ NMR (600 MHz, $\left.\mathrm{CDCl}_{3}\right) \delta 7.74$ (s, 1H), 7.66 (d, $J=7.8 \mathrm{~Hz}, 1 \mathrm{H}), 7.27-7.24(\mathrm{~m}, 2 \mathrm{H}), 7.25(\mathrm{~s}, 1 \mathrm{H}), 7.21(\mathrm{~d}, J=6.6 \mathrm{~Hz}, 2 \mathrm{H}), 7.19$ 7.15 (m, 2H), 7.13 (t, $J=7.2 \mathrm{~Hz}, 1 \mathrm{H}), 6.84(\mathrm{~s}, 1 \mathrm{H}), 6.09$ (s, 1H), 4.42 (t, $J=$ $6.6 \mathrm{~Hz}, 2 \mathrm{H}), 3.74(\mathrm{~s}, 3 \mathrm{H}), 2.99(\mathrm{t}, J=6.6 \mathrm{~Hz}, 2 \mathrm{H}) ;{ }^{13} \mathrm{C} \mathrm{NMR}\left(150 \mathrm{MHz}, \mathrm{CDCl}_{3}\right) \delta 165.7,137.3$, 136.3, 135.8, 131.7, 128.7, 128.3, 127.2, 126.5, 122.1, 119.8, 118.3, 109.2, 108.8, 104.0, 66.1, 35.1, 32.9; IR (ATR) 3398, 2933, 1676, 1456, 1329, 1233, 1121, 993, 847, 738, $646 \mathrm{~cm}^{-1}$; HRMS $m / z\left[\mathrm{M}^{+}\right]$calcd for $\mathrm{C}_{20} \mathrm{H}_{19} \mathrm{NO}_{3}: 321.1365$. Found: 321.1363 .

\section{3-Phenylpropyl (Z)-2-hydroxy-3-(1-methyl-1H-indol-3-yl)acrylate (3g)}

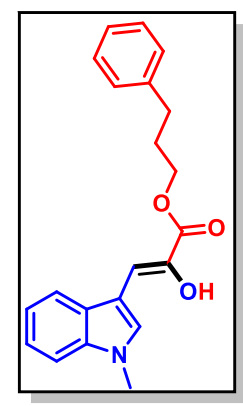

The title compound was prepared according to the general procedure. The product was obtained as a pink solid. EtOAc:Hex (1:9); Yield: 60\% (101 mg); mp 98-100 ${ }^{\circ} \mathrm{C} .{ }^{1} \mathrm{H}$ NMR $\left(600 \mathrm{MHz}, \mathrm{CDCl}_{3}\right) \delta 7.84(\mathrm{~s}, 1 \mathrm{H}), 7.77(\mathrm{~d}, J=6.6 \mathrm{~Hz}$, 1H), 7.34-7.25 (m, 4H), 7.24-7.18 (m, 4H), 6.93 (s, 1H), 6.19 (s, 1H), 4.32 (d, $J$ $=7.2 \mathrm{~Hz}, 2 \mathrm{H}), 3.83(\mathrm{~s}, 3 \mathrm{H}), 2.79(\mathrm{~d}, J=6.6 \mathrm{~Hz}, 2 \mathrm{H}), 2.12(\mathrm{~d}, J=6.0 \mathrm{~Hz}, 2 \mathrm{H})$;

${ }^{13} \mathrm{C}$ NMR $\left(150 \mathrm{MHz}, \mathrm{CDCl}_{3}\right) \delta 165.9,140.7,136.3,135.8,131.7,128.2,128.2$, 127.2, 125.8, 122.1, 119.8, 118.4, 109.2, 108.9, 103.7, 65.0, 32.9, 31.9, 30.0; IR (ATR) 3424, 2943, 1669, 1329, 1218, 971, $744516 \mathrm{~cm}^{-1}$; HRMS m/z [M+] calcd for $\mathrm{C}_{21} \mathrm{H}_{21} \mathrm{NO}_{3}: 335.1521$. Found: 335.1518.

\section{Ethyl (Z)-3-(1-ethyl-1H-indol-3-yl)-2-hydroxyacrylate (3h)}

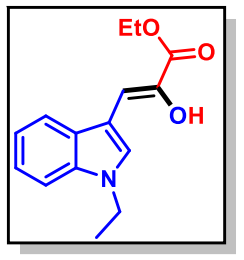

The title compound was prepared according to the general procedure. The product was obtained as a pink solid. EtOAc:Hex (2:8); Yield: 66\% (85 mg); mp 68-70 C. ${ }^{1} \mathrm{H}$ NMR $\left(600 \mathrm{MHz}, \mathrm{CDCl}_{3}\right) \delta 7.90(\mathrm{~s}, 1 \mathrm{H}), 7.79(\mathrm{~d}, J=7.8 \mathrm{~Hz}, 1 \mathrm{H})$, $7.35(\mathrm{~d}, J=7.8 \mathrm{~Hz}, 1 \mathrm{H}), 7.26(\mathrm{~d}, J=7.2 \mathrm{~Hz}, 1 \mathrm{H}), 7.19$ (t, $J=7.2 \mathrm{~Hz}, 1 \mathrm{H}), 6.95$ (s, 1H), $6.25(\mathrm{~s}, 1 \mathrm{H}), 4.37(\mathrm{q}, J=7.2 \mathrm{~Hz}, 2 \mathrm{H}), 4.20(\mathrm{q}, J=7.2 \mathrm{~Hz}, 2 \mathrm{H}), 1.49$ (t, $J=7.2 \mathrm{~Hz}, 3 \mathrm{H})$, 
$1.40(\mathrm{t}, J=6.6 \mathrm{~Hz}, 3 \mathrm{H}) ;{ }^{13} \mathrm{C} \mathrm{NMR}\left(150 \mathrm{MHz}, \mathrm{CDCl}_{3}\right) \delta 165.9,135.9,135.3,130.1,127.4,121.8$, 119.7, 118.5, 109.3, 109.0, 103.7, 61.7, 41.1, 15.1, 14.1; IR (ATR) 3456, 2922, 1723, 1461, 1364, 1216, 1065, 742, $423 \mathrm{~cm}^{-1}$; HRMS m/z $\left[\mathrm{M}^{+}\right]$calcd for $\mathrm{C}_{15} \mathrm{H}_{17} \mathrm{NO}_{3}$ : 259.1208. Found: 259.1207.

\section{Ethyl (Z)-2-hydroxy-3-(1-propyl-1H-indol-3-yl)acrylate (3i)}

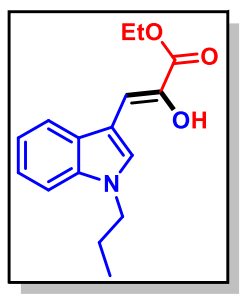

The title compound was prepared according to the general procedure. The product was obtained as a white solid. EtOAc:Hex (2:8); Yield: 60\% (82 mg); mp 108-110 ${ }^{\circ} \mathrm{C} .{ }^{1} \mathrm{H}$ NMR $\left(600 \mathrm{MHz}, \mathrm{CDCl}_{3}\right) \delta 7.88(\mathrm{~s}, 1 \mathrm{H}), 7.78(\mathrm{~d}, J=7.8 \mathrm{~Hz}$, $1 \mathrm{H}), 7.35(\mathrm{~d}, J=7.8 \mathrm{~Hz}, 1 \mathrm{H}), 7.24(\mathrm{t}, J=9.6 \mathrm{~Hz}, 1 \mathrm{H}), 7.19(\mathrm{t}, J=7.8 \mathrm{~Hz}, 1 \mathrm{H})$, $6.95(\mathrm{~s}, 1 \mathrm{H}), 6.23(\mathrm{~s}, 1 \mathrm{H}), 4.38$ (q, $J=7.8 \mathrm{~Hz}, 2 \mathrm{H}), 4.13(\mathrm{t}, J=7.2 \mathrm{~Hz}, 2 \mathrm{H})$, $1.91(\mathrm{q}, J=7.2 \mathrm{~Hz}, 2 \mathrm{H}), 1.41(\mathrm{t}, J=6.6 \mathrm{~Hz}, 3 \mathrm{H}), 0.96(\mathrm{t}, J=7.8 \mathrm{~Hz}, 3 \mathrm{H}) ;{ }^{13} \mathrm{C} \mathrm{NMR}(150 \mathrm{MHz}$, $\left.\mathrm{CDCl}_{3}\right) \delta 166.1,136.1,135.9,131.0,127.6,122.0,119.9,118.7,109.6,109.0,103.9,61.9,48.4$, 23.4, 14.3, 11.5; IR (ATR) 3387, 2963, 1652, 1455, 1338, 1221, 1017, 741, 642, $517 \mathrm{~cm}^{-1}$; HRMS $m / z,\left[\mathrm{M}^{+}\right]$calcd for $\mathrm{C}_{16} \mathrm{H}_{19} \mathrm{NO}_{3}: 273.1365$. Found: 273.1366 .

\section{Ethyl (Z)-3-(1-butyl-1H-indol-3-yl)-2-hydroxyacrylate (3j)}

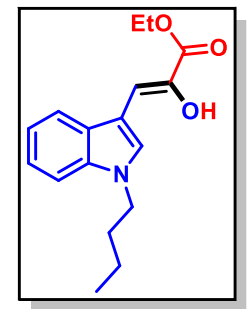

The title compound was prepared according to the general procedure. The product was obtained as a pink solid. EtOAc:Hex (1:9); Yield: 61\% (88 mg); mp 123-125 ${ }^{\circ} \mathrm{C} .{ }^{1} \mathrm{H}$ NMR $\left(600 \mathrm{MHz}, \mathrm{CDCl}_{3}\right) \delta 7.86(\mathrm{~s}, 1 \mathrm{H}), 7.77(\mathrm{~d}, J=7.8 \mathrm{~Hz}$, $1 \mathrm{H}), 7.34(\mathrm{~d}, J=8.4 \mathrm{~Hz}, 1 \mathrm{H}), 7.24(\mathrm{~d}, J=7.8 \mathrm{~Hz}, 1 \mathrm{H}), 7.18(\mathrm{~d}, J=7.8 \mathrm{~Hz}, 1 \mathrm{H})$, $6.94(\mathrm{~s}, 1 \mathrm{H}), 6.23(\mathrm{~s}, 1 \mathrm{H}), 4.37$ (q, $J=7.2 \mathrm{~Hz}, 2 \mathrm{H}), 4.14(\mathrm{t}, J=7.2 \mathrm{~Hz}, 2 \mathrm{H})$, $1.86(\mathrm{q}, J=7.2 \mathrm{~Hz}, 2 \mathrm{H}), 1.40(\mathrm{t}, J=7.2 \mathrm{~Hz}, 3 \mathrm{H}), 1.35$ (q, $J=7.2 \mathrm{~Hz}, 2 \mathrm{H}), 0.93$ (t, $J=7.2 \mathrm{~Hz}$, $3 \mathrm{H}) ;{ }^{13} \mathrm{C} \mathrm{NMR}\left(150 \mathrm{MHz}, \mathrm{CDCl}_{3}\right) \delta 165.9,135.9,135.6,130.7,127.3,121.8,119.7,118.5$, 109.4, 108.8, 103.7, 61.7, 46.2, 31.9, 19.9, 14.1, 13.4; IR (ATR) 3374, 2946, 1656, 1125, 1018, 742, 646, $521 \mathrm{~cm}^{-1}$; HRMS m/z $\left[\mathrm{M}^{+}\right]$calcd for $\mathrm{C}_{17} \mathrm{H}_{21} \mathrm{NO}_{3}: 287.1521$. Found: 287.1519.

\section{Ethyl (Z)-2-hydroxy-3-(1-pentyl-1H-indol-3-yl)acrylate (3k)}

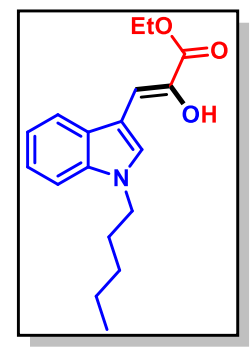

The title compound was prepared according to the general procedure. The product was obtained as a pink solid. EtOAc:Hex (2:8); Yield: 67\% (101 mg); 
mp 82-84 ${ }^{\circ} \mathrm{C} .{ }^{1} \mathrm{H}$ NMR $\left(600 \mathrm{MHz}, \mathrm{CDCl}_{3}\right) \delta 7.86(\mathrm{~s}, 1 \mathrm{H}), 7.78(\mathrm{~d}, J=7.8 \mathrm{~Hz}, 1 \mathrm{H}), 7.34(\mathrm{~d}, J=$ $7.8 \mathrm{~Hz}, 1 \mathrm{H}), 7.24(\mathrm{t}, J=9.0 \mathrm{~Hz}, 1 \mathrm{H}), 7.18(\mathrm{t}, J=7.8 \mathrm{~Hz}, 1 \mathrm{H}), 6.94(\mathrm{~s}, 1 \mathrm{H}), 6.24(\mathrm{~s}, 1 \mathrm{H}), 4.36$ (q, $J=7.2 \mathrm{~Hz}, 2 \mathrm{H}), 4.13(\mathrm{t}, J=7.2 \mathrm{~Hz}, 2 \mathrm{H}), 1.86(\mathrm{p}, J=7.2 \mathrm{~Hz}, 2 \mathrm{H}), 1.40(\mathrm{t}, J=7.2 \mathrm{~Hz}, 3 \mathrm{H}), 1.32$ $(\mathrm{tdd}, J=12.6,7.2,2.4 \mathrm{~Hz}, 4 \mathrm{H}), 0.87(\mathrm{t}, J=6.6 \mathrm{~Hz}, 3 \mathrm{H}) ;{ }^{13} \mathrm{C} \mathrm{NMR}\left(150 \mathrm{MHz}, \mathrm{CDCl}_{3}\right) \delta 165.9$, 135.9, 135.6, 130.7, 127.3, 121.8, 119.7, 118.5, 109.4, 108.8, 103.7, 61.7, 46.5, 29.5, 28.8, 22.1, 14.1, 13.6; IR (ATR) 3384, 2927, 1731, 1651, 1455, 1363, 1221, 1018, 740, $521 \mathrm{~cm}^{-1}$; HRMS $m / z\left[\mathrm{M}^{+}\right]$calcd for $\mathrm{C}_{18} \mathrm{H}_{23} \mathrm{NO}_{3}: 301.1678$. Found: 301.1675 .

\section{Ethyl (Z)-3-(1-(3-chloropropyl)-1H-indol-3-yl)-2-hydroxyacrylate (3l)}

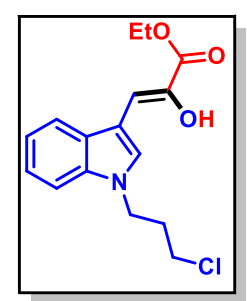

The title compound was prepared according to the general procedure. The product was obtained as a white solid. EtOAc:Hex (2:8); Yield: 55\% (84 mg); mp 98-100 C. ${ }^{1} \mathrm{H}$ NMR (600 MHz, $\left.\mathrm{CDCl}_{3}\right) \delta 7.88(\mathrm{~s}, 1 \mathrm{H}), 7.78(\mathrm{~d}, J=7.8 \mathrm{~Hz}$, $1 \mathrm{H}), 7.38(\mathrm{~d}, J=8.2 \mathrm{~Hz}, 1 \mathrm{H}), 7.28-7.24(\mathrm{~m}, 1 \mathrm{H}), 7.21-7.17(\mathrm{~m}, 1 \mathrm{H}), 6.92(\mathrm{~s}$, $1 \mathrm{H}), 6.27(\mathrm{~s}, 1 \mathrm{H}), 4.38-4.34(\mathrm{~m}, 4 \mathrm{H}), 3.47(\mathrm{t}, J=6.0 \mathrm{~Hz}, 2 \mathrm{H}), 2.30(\mathrm{p}, J=6.3$ $\mathrm{Hz}, 2 \mathrm{H}), 1.40(\mathrm{t}, J=7.1 \mathrm{~Hz}, 3 \mathrm{H}) ;{ }^{13} \mathrm{C} \mathrm{NMR}\left(150 \mathrm{MHz}, \mathrm{CDCl}_{3}\right) \delta 165.8,136.3,135.5,130.6$, $127.4,122.2$, 120.0, 118.6, 109.5, 109.2, 103.3, 61.8, 43.0, 41.5, 32.3, 14.1; IR (ATR) 3371, 2957, 1654, 1340, 1228, 1017, 742, 646, $519 \mathrm{~cm}^{-1}$; HRMS m/z $\left[\mathrm{M}^{+}\right]$calcd for $\mathrm{C}_{16} \mathrm{H}_{18} \mathrm{ClNO}_{3}$ : 307.0975. Found: 307.0977.

\section{Ethyl (Z)-3-(1-benzyl-1H-indol-3-yl)-2-hydroxyacrylate (3m)}

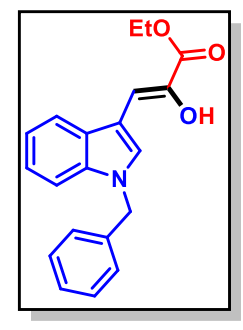

The title compound was prepared according to the general procedure. The product was obtained as a white solid. EtOAc:Hex (1:9); Yield: 64\% (103 mg); mp 120-122 ${ }^{\circ} \mathrm{C} .{ }^{1} \mathrm{H}$ NMR $\left(600 \mathrm{MHz}, \mathrm{CDCl}_{3}\right) \delta 7.91(\mathrm{~s}, 1 \mathrm{H}), 7.79$ (d, $J=9.6 \mathrm{~Hz}$, 1H), 7.29-7.23 (m, 4H), 7.20-7.15 (m, 2H), $7.12(\mathrm{~d}, J=6.6 \mathrm{~Hz}, 2 \mathrm{H}), 6.94(\mathrm{~s}, 1 \mathrm{H})$, $6.24(\mathrm{~s}, 1 \mathrm{H}), 5.33(\mathrm{~s}, 2 \mathrm{H}), 4.35(\mathrm{q}, J=7.2 \mathrm{~Hz}, 2 \mathrm{H}), 1.39(\mathrm{t}, J=7.2 \mathrm{~Hz}, 3 \mathrm{H}) ;{ }^{13} \mathrm{C}$ NMR $\left(150 \mathrm{MHz}, \mathrm{CDCl}_{3}\right) \delta 165.8,136.6,136.2,135.8,131.2,128.5,127.5,127.5,126.5,122.2$, 120.0, 118.5, 109.8, 109.5, 103.4, 61.8, 50.2, 14.1; IR (ATR) 3425, 2975, 1675, 1339, 1215, 1108, 866, 696, $517 \mathrm{~cm}^{-1}$; HRMS m/z [ $\left.\mathrm{M}^{+}\right]$calcd for $\mathrm{C}_{20} \mathrm{H}_{19} \mathrm{NO}_{3}: 321.1365$. Found: 321.1364 . 


\section{Ethyl (Z)-2-hydroxy-3-(1-(1-phenylethyl)-1H-indol-3-yl)acrylate (3n)}

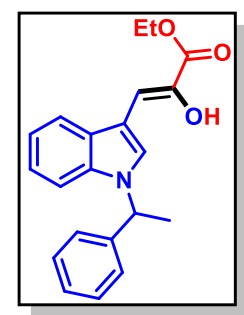

The title compound was prepared according to the general procedure. The product was obtained as a yellow solid. EtOAc:Hex (2:8); Yield: 77\% (129 mg); mp 85$87^{\circ} \mathrm{C} .{ }^{1} \mathrm{H}$ NMR $\left(600 \mathrm{MHz}, \mathrm{CDCl}_{3}\right) \delta 8.16(\mathrm{~s}, 1 \mathrm{H}), 7.86(\mathrm{~d}, J=7.2 \mathrm{~Hz}, 1 \mathrm{H}), 7.33$ $(\mathrm{t}, J=7.8 \mathrm{~Hz}, 2 \mathrm{H}), 7.28-7.25(\mathrm{~m}, 2 \mathrm{H}), 7.23-7.19(\mathrm{~m}, 4 \mathrm{H}), 7.06(\mathrm{~s}, 1 \mathrm{H}), 6.38(\mathrm{~s}$, $1 \mathrm{H}), 5.72(\mathrm{q}, J=6.6 \mathrm{~Hz}, 1 \mathrm{H}), 4.44(\mathrm{q}, J=7.2 \mathrm{~Hz}, 2 \mathrm{H}), 2.00(\mathrm{~d}, J=7.2 \mathrm{~Hz}, 3 \mathrm{H}), 1.46$ (t, $J=7.2$ $\mathrm{Hz}, 3 \mathrm{H}) ;{ }^{13} \mathrm{C} \mathrm{NMR}\left(150 \mathrm{MHz}, \mathrm{CDCl}_{3}\right) \delta 165.8,141.8,136.0,135.6,128.5,127.9,127.6,127.2$, $125.5,121.9$, 120.0, 118.4, 110.2, 109.3, 103.6, 61.7, 55.1, 21.5, 14.1; IR (ATR) 3461, 2988, 1723, 1458, 1181, $742 \mathrm{~cm}^{-1}$; HRMS m/z $\left[\mathrm{M}^{+}\right]$calcd for $\mathrm{C}_{21} \mathrm{H}_{21} \mathrm{NO}_{3}: 335.1521$. Found: 335.1518 .

\section{Ethyl (Z)-3-(1-allyl-1H-indol-3-yl)-2-hydroxyacrylate (3o)}

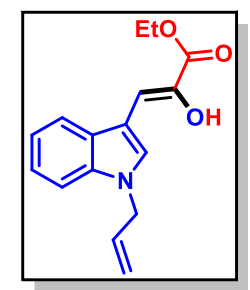

The title compound was prepared according to the general procedure. The product was obtained as a pink solid. EtOAc:Hex (1:9); Yield: 63\% (85 mg); mp 100-102 ${ }^{\circ} \mathrm{C} .{ }^{1} \mathrm{H}$ NMR $\left(300 \mathrm{MHz}, \mathrm{CDCl}_{3}\right) \delta 7.94(\mathrm{~s}, 1 \mathrm{H}), 7.86(\mathrm{~d}, J=7.8 \mathrm{~Hz}$, $1 \mathrm{H}), 7.40(\mathrm{~d}, J=7.8 \mathrm{~Hz}, 1 \mathrm{H}), 7.26(\mathrm{t}, J=6.6 \mathrm{~Hz}, 2 \mathrm{H}), 7.02(\mathrm{~s}, 1 \mathrm{H}), 6.34(\mathrm{~s}$, 1H), 5.98 (p, $J=2.4,1.5 \mathrm{~Hz}, 1 \mathrm{H}), 5.29$ (d, $J=10.2 \mathrm{~Hz}, 1 \mathrm{H}), 5.19$ (d, $J=17.1$ $\mathrm{Hz}, 1 \mathrm{H}), 4.84(\mathrm{~s}, 2 \mathrm{H}), 4.44(\mathrm{q}, J=6.6 \mathrm{~Hz}, 2 \mathrm{H}), 1.48(\mathrm{t}, J=6.6 \mathrm{~Hz}, 3 \mathrm{H}) ;{ }^{13} \mathrm{C} \mathrm{NMR}(75 \mathrm{MHz}$, $\left.\mathrm{CDCl}_{3}\right) \delta 165.6,135.9,135.4,132.4,130.5,127.2,121.8,119.7,118.3,117.2,109.4,109.1$, 103.2, 61.5, 48.7, 13.9; IR (ATR) 3407, 2982, 1724, 1660, 1458, 1339, 1234, 1177, 919, 738, $642 \mathrm{~cm}^{-1}$; HRMS m/z $\left[\mathrm{M}^{+}\right]$calcd for $\mathrm{C}_{16} \mathrm{H}_{17} \mathrm{NO}_{3}: 271.1208$. Found: 271.1206.

\section{Isopropyl (Z)-2-hydroxy-3-(1-phenyl-1H-indol-3-yl)acrylate (3p)}

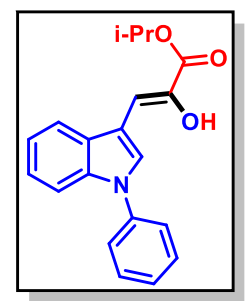

The title compound was prepared according to the general procedure. The product was obtained as a brown solid. EtOAc:Hex (2:8); Yield: 59\% (95 mg); mp 79-81 C. ${ }^{1} \mathrm{H}$ NMR $\left(600 \mathrm{MHz}, \mathrm{CDCl}_{3}\right) \delta 8.09$ (s, 1H), $7.84(\mathrm{~d}, J=9.0 \mathrm{~Hz}$, 1H), 7.55-7.49 (m, 6H), $7.37(\mathrm{t}, J=6.6 \mathrm{~Hz}, 1 \mathrm{H}), 7.25(\mathrm{~d}, J=6.6 \mathrm{~Hz}, 1 \mathrm{H}), 6.93$ $(\mathrm{s}, 1 \mathrm{H}), 6.35(\mathrm{~s}, 1 \mathrm{H}), 5.20(\mathrm{p}, J=6.0 \mathrm{~Hz}, 1 \mathrm{H}), 1.39(\mathrm{~d}, J=6.6 \mathrm{~Hz}, 6 \mathrm{H}) ;{ }^{13} \mathrm{C}$ NMR $\left(150 \mathrm{MHz}, \mathrm{CDCl}_{3}\right) \delta 165.3,139.1,137.3,135.5,130.3,129.4,128.0,126.6,124.2,122.7$, 120.6, 118.7, 111.1, 110.5, 102.5, 69.8, 21.7; IR (ATR) 3489, 2927, 1715, 1371, 1091, 745, 487 $\mathrm{cm}^{-1}$; HRMS $\mathrm{m} / z\left[\mathrm{M}^{+}\right]$calcd for $\mathrm{C}_{20} \mathrm{H}_{19} \mathrm{NO}_{3}$ : 321.1365 . Found: 321.1362 . 


\section{Isopropyl (Z)-3-(1-acetyl-1H-indol-3-yl)-2-hydroxyacrylate (3q)}

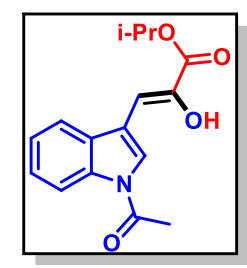

The title compound was prepared according to the general procedure. The product was obtained as a pale yellow solid. EtOAc:Hex (2:8); Yield: 44\% (63 $\mathrm{mg})$; mp 128-130 C. ${ }^{1} \mathrm{H}$ NMR (600 MHz, $\left.\mathrm{CDCl}_{3}\right) \delta 8.47(\mathrm{~d}, J=8.4 \mathrm{~Hz}, 1 \mathrm{H})$, 8.09 (s, 1H), 7.69 (d, $J=7.8 \mathrm{~Hz}, 1 \mathrm{H}), 7.38$ (t, $J=7.8 \mathrm{~Hz}, 1 \mathrm{H}), 7.33$ (t, $J=7.8$ $\mathrm{Hz}, 1 \mathrm{H}), 6.74(\mathrm{~s}, 1 \mathrm{H}), 6.60(\mathrm{~s}, 1 \mathrm{H}), 5.23-5.181(\mathrm{~m}, 1 \mathrm{H}), 2.67(\mathrm{~s}, 3 \mathrm{H}), 1.38$ (d, J = 6.0 Hz, 6H); ${ }^{13} \mathrm{C} \mathrm{NMR}\left(150 \mathrm{MHz}, \mathrm{CDCl}_{3}\right) \delta 168.9,165.0,139.9,135.3,129.4,126.7,125.6,123.8,118.4$, 116.7, 115.8, 100.5, 70.7, 24.0, 21.8; IR (ATR) 3374, 2925, 1691, 1452, 1369, 1241, 1069, 926, 745, $655 \mathrm{~cm}^{-1}$; HRMS m/z $\left[\mathrm{M}^{+}\right]$calcd for $\mathrm{C}_{16} \mathrm{H}_{17} \mathrm{NO}_{4}: 287.1158$. Found: 287.1157.

\section{Ethyl (Z)-3-(4-(benzyloxy)-1-methyl-1H-indol-3-yl)-2-hydroxyacrylate (4a)}

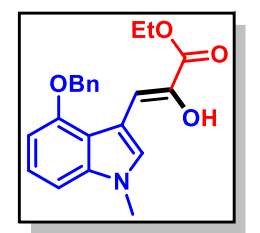

The title compound was prepared according to the general procedure. The product was obtained as a offwhite solid. EtOAc:Hex (1:9); Yield: $55 \%$ (97 mg); mp 165-167 ${ }^{\circ} \mathrm{C} .{ }^{1} \mathrm{H}$ NMR $\left(600 \mathrm{MHz}, \mathrm{CDCl}_{3}\right) \delta 7.81(\mathrm{~s}, 1 \mathrm{H}), 7.57(\mathrm{~s}, 1 \mathrm{H}), 7.54(\mathrm{~d}$, $J=7.2 \mathrm{~Hz}, 2 \mathrm{H}), 7.39(\mathrm{t}, J=7.8 \mathrm{~Hz}, 2 \mathrm{H}), 7.33(\mathrm{~d}, J=6.0 \mathrm{~Hz}, 1 \mathrm{H}), 7.16(\mathrm{t}, J=$ $8.4 \mathrm{~Hz}, 1 \mathrm{H}), 6.95(\mathrm{~d}, J=8.4 \mathrm{~Hz}, 1 \mathrm{H}), 6.68(\mathrm{~d}, J=7.8 \mathrm{~Hz}, 1 \mathrm{H}), 6.21(\mathrm{~s}, 1 \mathrm{H}), 5.22(\mathrm{~s}, 2 \mathrm{H}), 4.22$ (q, $J=7.2 \mathrm{~Hz}, 2 \mathrm{H}), 3.79(\mathrm{~s}, 3 \mathrm{H}), 1.16(\mathrm{t}, J=7.2 \mathrm{~Hz}, 3 \mathrm{H}) ;{ }^{13} \mathrm{C} \mathrm{NMR}\left(150 \mathrm{MHz}, \mathrm{CDCl}_{3}\right) \delta 166.5$, 154.1, 138.2, 137.2, 135.7, 131.3, 128.4, 127.7, 127.6, 122.8, 116.9, 109.5, 106.6, 103.1, 101.8, 70.2, 61.6, 33.3, 14.3; IR (ATR) 3432, 2936, 1668, 1323, 1214, 1014, 726, $636 \mathrm{~cm}^{-1}$; HRMS m/z $\left[\mathrm{M}^{+}\right]$calcd for $\mathrm{C}_{21} \mathrm{H}_{21} \mathrm{NO}_{4}$ : 351.1471. Found: 351.1474 .

\section{Ethyl (Z)-2-hydroxy-3-(1-methyl-4-phenyl-1H-indol-3-yl)acrylate (4b)}

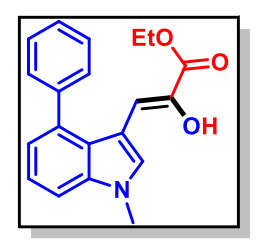

The title compound was prepared according to the general procedure. The product was obtained as a light brown solid. EtOAc:Hex (1:9); Yield: 50\% (80 $\mathrm{mg}) ; \mathrm{mp} 89-91{ }^{\circ} \mathrm{C} .{ }^{1} \mathrm{H}$ NMR $\left(600 \mathrm{MHz}, \mathrm{CDCl}_{3}\right) \delta 7.93(\mathrm{~s}, 1 \mathrm{H}), 7.47(\mathrm{~d}, J=6.6$ $\mathrm{Hz}, 2 \mathrm{H}), 7.43(\mathrm{t}, J=7.8 \mathrm{~Hz}, 2 \mathrm{H}), 7.38(\mathrm{t}, J=7.2 \mathrm{~Hz}, 1 \mathrm{H}), 7.32(\mathrm{~d}, J=8.4 \mathrm{~Hz}$, $1 \mathrm{H}), 7.28(\mathrm{t}, J=7.2 \mathrm{~Hz}, 1 \mathrm{H}), 7.03(\mathrm{~d}, J=7.2 \mathrm{~Hz}, 1 \mathrm{H}), 6.29$ (s, 1H), 6.10 (s, 1H), 4.10 (q, $J=7.2$ $\mathrm{Hz}, 2 \mathrm{H}), 3.86(\mathrm{~s}, 3 \mathrm{H}), 1.19(\mathrm{t}, J=7.2 \mathrm{~Hz}, 3 \mathrm{H}) ;{ }^{13} \mathrm{C} \mathrm{NMR}\left(150 \mathrm{MHz}, \mathrm{CDCl}_{3}\right) \delta 166.1,141.0$, $136.7,135.7,134.8,132.8,129.3,127.7,126.8,124.3,121.8,121.6,109.1,108.3,105.3,61.3$, 
33.1, 13.9; IR (ATR) 3451, 2926, 1721, 1455, 1229, 1162, 749, $699 \mathrm{~cm}^{-1} ;$ HRMS $\mathrm{m} / z$ [M+c calcd for $\mathrm{C}_{20} \mathrm{H}_{19} \mathrm{NO}_{3}$ : 321.1365. Found: 321.1364 .

\section{Butyl (Z)-2-hydroxy-3-(1-methyl-4-phenyl-1H-indol-3-yl)acrylate (4c)}

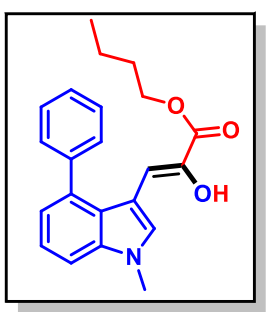

The title compound was prepared according to the general procedure. The product was obtained as a pale yellow solid. EtOAc:Hex (1:9); Yield: 56\% (98 $\mathrm{mg}$ ); mp 115-117 ${ }^{\circ} \mathrm{C} .{ }^{1} \mathrm{H}$ NMR (600 MHz, $\left.\mathrm{CDCl}_{3}\right) \delta 7.95$ (s, 1H), 7.47 (d, $J=$ $6.6 \mathrm{~Hz}, 2 \mathrm{H}), 7.43(\mathrm{t}, J=7.2 \mathrm{~Hz}, 2 \mathrm{H}), 7.37(\mathrm{t}, J=7.2 \mathrm{~Hz}, 1 \mathrm{H}), 7.32(\mathrm{~d}, J=7.8$ $\mathrm{Hz}, 1 \mathrm{H}), 7.28(\mathrm{t}, J=6.6 \mathrm{~Hz}, 1 \mathrm{H}), 7.02(\mathrm{~d}, J=6.6 \mathrm{~Hz}, 1 \mathrm{H}), 6.30(\mathrm{~s}, 1 \mathrm{H}), 6.15$ (s, 1H), $4.06(\mathrm{t}, J=6.6 \mathrm{~Hz}, 2 \mathrm{H}), 3.86(\mathrm{~s}, 3 \mathrm{H}), 1.54-1.50(\mathrm{~m}, 2 \mathrm{H}), 1.28(\mathrm{q}, J=7.8 \mathrm{~Hz}, 2 \mathrm{H}), 0.94$ (t, $J=7.2 \mathrm{~Hz}, 3 \mathrm{H}) ;{ }^{13} \mathrm{C}$ NMR $\left(150 \mathrm{MHz}, \mathrm{CDCl}_{3}\right) \delta 166.2,141.0,136.7,135.7,134.9,132.9$, 129.3, 127.7, 126.8, 124.3, 121.9, 121.6, 109.1, 108.3, 105.2, 65.3, 33.1, 30.3, 18.8, 13.5; IR (ATR) 3411, 2957, 1664, 1402, 1317, 1225, 1119, 1052, 963, 744, 646, $518 \mathrm{~cm}^{-1}$ HRMS m/z $\left[\mathrm{M}^{+}\right]$calcd for $\mathrm{C}_{22} \mathrm{H}_{23} \mathrm{NO}_{3}$ : 349.1678. Found: 349.1676.

\section{Ethyl (Z)-3-(1,5-dimethyl-1H-indol-3-yl)-2-hydroxyacrylate (4d)}

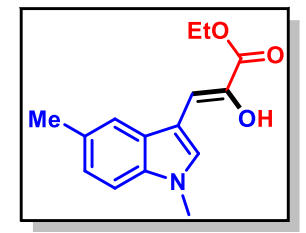

The title compound was prepared according to the general procedure. The product was obtained as a yellow solid. EtOAc:Hex (2:8); Yield: 63\% (82 $\mathrm{mg}$ ); mp 165-167 ${ }^{\circ} \mathrm{C} .{ }^{1} \mathrm{H}$ NMR $\left(600 \mathrm{MHz}, \mathrm{CDCl}_{3}\right) \delta 7.78$ (s, 1H), 7.55 (s, 1H), $7.20(\mathrm{~d}, J=7.8 \mathrm{~Hz}, 1 \mathrm{H}), 7.08(\mathrm{~d}, J=9 \mathrm{~Hz}, 1 \mathrm{H}), 6.91(\mathrm{~s}, 1 \mathrm{H}), 6.22(\mathrm{~s}$, $1 \mathrm{H}), 4.35$ (q, $J=7.2 \mathrm{~Hz}, 2 \mathrm{H}), 3.79$ (s, 3H), $2.48(\mathrm{~s}, 3 \mathrm{H}), 1.40(\mathrm{t}, J=7.2 \mathrm{~Hz}, 3 \mathrm{H}) ;{ }^{13} \mathrm{C}$ NMR $(150$ $\left.\mathrm{MHz}, \mathrm{CDCl}_{3}\right) \delta 166.0,135.7,134.7,131.8,129.2,127.5,123.6,118.2,108.9,108.4,103.9,61.7$,

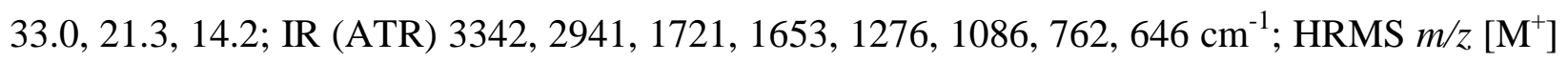
calcd for $\mathrm{C}_{15} \mathrm{H}_{17} \mathrm{NO}_{3}: 259.1208$. Found: 259.1206.

\section{Ethyl (Z)-2-hydroxy-3-(5-methoxy-1-methyl-1H-indol-3-yl)acrylate (4e)}

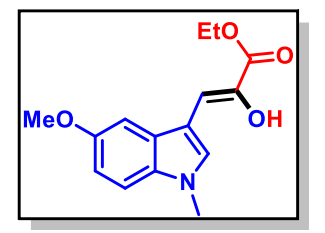

The title compound was prepared according to the general procedure. The product was obtained as a light green solid. EtOAc:Hex (2:8); Yield: 54\% (74 mg); mp 143-145 ${ }^{\circ} \mathrm{C} .{ }^{1} \mathrm{H}$ NMR (600 MHz, $\left.\mathrm{CDCl}_{3}\right) \delta 7.79$ (s, 1H), 7.20 (s, $1 \mathrm{H}), 7.19(\mathrm{~d}, J=6.8 \mathrm{~Hz}, 1 \mathrm{H}), 6.91(\mathrm{dd}, J=9.0,2.4 \mathrm{~Hz}, 1 \mathrm{H}), 6.87(\mathrm{~s}, 1 \mathrm{H})$, $6.22(\mathrm{~s}, 1 \mathrm{H}), 4.37$ (q, $J=7.2 \mathrm{~Hz}, 2 \mathrm{H}), 3.89$ (s, 3H), $3.78(\mathrm{~s}, 3 \mathrm{H}), 1.40(\mathrm{t}, J=7.2 \mathrm{~Hz}, 3 \mathrm{H}) ;{ }^{13} \mathrm{C}$ 
NMR $\left(150 \mathrm{MHz}, \mathrm{CDCl}_{3}\right) \delta 165.9,154.5,135.7,132.3,131.7,127.8,112.2,110.1,108.6,103.9$, 100.5, 61.8, 55.8, 33.1, 14.2; IR (ATR) 3398, 2936, 1551, 1684, 1315, 1218, 763, $642 \mathrm{~cm}^{-1}$; HRMS $m / z\left[\mathrm{M}^{+}\right]$calcd for $\mathrm{C}_{15} \mathrm{H}_{17} \mathrm{NO}_{4}$ : 275.1158. Found: 275.1156 .

Ethyl (Z)-2-hydroxy-3-(1-methyl-5-phenyl-1H-indol-3-yl)acrylate (4f)

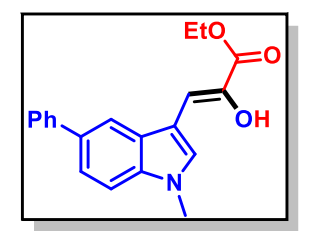

The title compound was prepared according to the general procedure. The product was obtained as a yellow solid. EtOAc:Hex (1:9); Yield: 57\% (91 $\mathrm{mg}) ; \mathrm{mp} 183-185^{\circ} \mathrm{C} .{ }^{1} \mathrm{H} \mathrm{NMR}\left(600 \mathrm{MHz}, \mathrm{CDCl}_{3}\right) \delta 7.96(\mathrm{~s}, 1 \mathrm{H}), 7.86(\mathrm{~s}, 1 \mathrm{H})$, $7.68(\mathrm{~d}, J=6.6 \mathrm{~Hz}, 2 \mathrm{H}), 7.51(\mathrm{dd}, J=7.2,1.8 \mathrm{~Hz}, 1 \mathrm{H}), 7.45(\mathrm{t}, J=7.8 \mathrm{~Hz}$, 2H), $7.37(\mathrm{~d}, J=9.0 \mathrm{~Hz}, 1 \mathrm{H}), 7.32(\mathrm{t}, J=7.2 \mathrm{~Hz}, 1 \mathrm{H}), 6.98(\mathrm{~s}, 1 \mathrm{H}), 6.29(\mathrm{~s}, 1 \mathrm{H}), 4.38(\mathrm{q}, J=6.6$ $\mathrm{Hz}, 2 \mathrm{H}), 3.84(\mathrm{~s}, 3 \mathrm{H}), 1.41(\mathrm{t}, J=7.2 \mathrm{~Hz}, 3 \mathrm{H}) ;{ }^{13} \mathrm{C} \mathrm{NMR}\left(150 \mathrm{MHz}, \mathrm{CDCl}_{3}\right) \delta 165.9,142.1$, $136.1,135.8,133.5,132.3,128.4,127.7,127.2,126.2$, 121.8, 117.1, 109.4, 109.3, 103.4, 61.8, 33.0, 14.1; IR (ATR) 3395, 2959, 1677, 1307, 1230, 1110, 865, 762, $509 \mathrm{~cm}^{-1}$; HRMS m/z [M+] calcd for $\mathrm{C}_{20} \mathrm{H}_{19} \mathrm{NO}_{3}$ : 321.1365 . Found: 321.1368 .

Ethyl (Z)-2-hydroxy-3-(1-methyl-5-(naphthalen-2-yl)-1H-indol-3-yl)acrylate (4g)

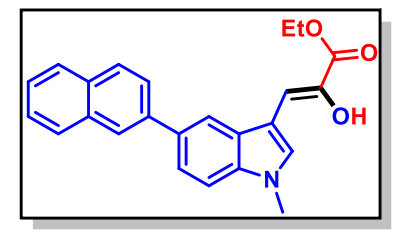

The title compound was prepared according to the general procedure. The product was obtained as a yellowish brown solid. EtOAc:Hex (2:8); Yield: 52\% (96 mg); mp 150-152 ${ }^{\circ} \mathrm{C}$. ${ }^{1} \mathrm{H}$ NMR (600 MHz, $\left.\mathrm{CDCl}_{3}\right) \delta$ $8.09(\mathrm{~d}, J=17.4 \mathrm{~Hz}, 2 \mathrm{H}), 7.91(\mathrm{t}, J=8.4 \mathrm{~Hz}, 2 \mathrm{H}), 7.87(\mathrm{~s}, 1 \mathrm{H}), 7.85(\mathrm{t}$, $J=7.8 \mathrm{~Hz}, 2 \mathrm{H}), 7.64(\mathrm{~d}, J=7.2 \mathrm{~Hz}, 1 \mathrm{H}), 7.46(\mathrm{t}, J=7.2 \mathrm{~Hz}, 1 \mathrm{H}), 7.45(\mathrm{t}, J=7.0 \mathrm{~Hz}, 1 \mathrm{H}), 7.42$ $(\mathrm{d}, J=8.4 \mathrm{~Hz}, 1 \mathrm{H}), 7.00(\mathrm{~s}, 1 \mathrm{H}), 6.27(\mathrm{~s}, 1 \mathrm{H}), 4.39(\mathrm{q}, J=7.2 \mathrm{~Hz}, 2 \mathrm{H}), 3.87(\mathrm{~s}, 3 \mathrm{H}), 1.41(\mathrm{t}, J=$ $7.2 \mathrm{~Hz}, 3 \mathrm{H}) ;{ }^{13} \mathrm{C} \mathrm{NMR}\left(150 \mathrm{MHz}, \mathrm{CDCl}_{3}\right) \delta 165.9,139.7,136.4,136.1,133.8,133.6,132.6$, 132.2, 128.2, 128.1, 128.0, 127.6, 126.2, 126.1, 125.6, 125.5, 122.3, 117.6, 109.8, 109.6, 103.6, 62.1, 33.3, 14.4; IR (ATR) 3298, 2927, 1678, 1240, 1116, 857, 796, 669, $469 \mathrm{~cm}^{-1}$; HRMS m/z $\left[\mathrm{M}^{+}\right]$calcd for $\mathrm{C}_{24} \mathrm{H}_{21} \mathrm{NO}_{3}: 371.1521$. Found: 371.1523 .

\section{Ethyl (Z)-3-(1,6-dimethyl-1H-indol-3-yl)-2-hydroxyacrylate (4h)}

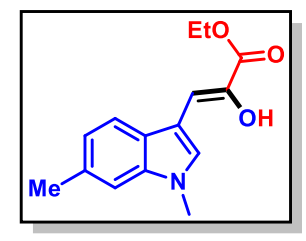

The title compound was prepared according to the general procedure. The product was obtained as a light brown solid. EtOAc:Hex (2:8); Yield: 60\% 
(78 mg); mp 101-103 ${ }^{\circ} \mathrm{C} .{ }^{1} \mathrm{H}$ NMR (600 MHz, $\left.\mathrm{CDCl}_{3}\right) \delta 7.88(\mathrm{~s}, 1 \mathrm{H}), 7.78(\mathrm{~d}, J=8.4 \mathrm{~Hz}, 1 \mathrm{H})$, $7.37(\mathrm{~s}, 1 \mathrm{H}), 7.15(\mathrm{~d}, J=9.0 \mathrm{~Hz}, 1 \mathrm{H}), 7.04(\mathrm{~s}, 1 \mathrm{H}), 6.33(\mathrm{~s}, 1 \mathrm{H}), 4.48$ (q, J = 6.6 Hz, 2H), 3.91 (s, $3 \mathrm{H}), 2.63(\mathrm{~s}, 3 \mathrm{H}), 1.52(\mathrm{t}, J=7.2 \mathrm{~Hz}, 3 \mathrm{H}) ;{ }^{13} \mathrm{C} \mathrm{NMR}\left(150 \mathrm{MHz}, \mathrm{CDCl}_{3}\right) \delta 165.9,136.7,135.8$, 131.9, 131.3, 125.2, 121.6, 118.2, 109.2, 108.8, 103.8, 61.7, 32.8, 21.6, 14.2; IR (ATR) 3418, 2930, 1726, 1374, 1228, 803, $595 \mathrm{~cm}^{-1}$; HRMS $m / z$ [ $\left.\mathrm{M}^{+}\right]$calcd for $\mathrm{C}_{15} \mathrm{H}_{17} \mathrm{NO}_{3}: 259.1208$. Found: 259.1205 .

Ethyl (Z)-3-(6-bromo-1-methyl-1H-indol-3-yl)-2-hydroxyacrylate (4i)

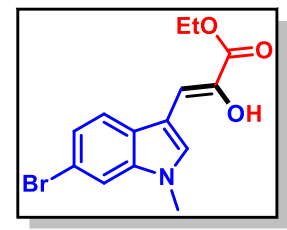

The title compound was prepared according to the general procedure. The product was obtained as a pink solid. EtOAc:Hex (2:8); Yield: 70\% (113 mg); mp 125-127 ${ }^{\circ}$ C. ${ }^{1} \mathrm{H}$ NMR $\left(600 \mathrm{MHz}, \mathrm{CDCl}_{3}\right) \delta 7.77(\mathrm{~s}, 1 \mathrm{H}), 7.61(\mathrm{~d}, J=8.4$ $\mathrm{Hz}, 1 \mathrm{H}), 7.46$ (s, 1H), 7.27 (dd, $J=8.4,1.2 \mathrm{~Hz}, 1 \mathrm{H}), 6.83(\mathrm{~s}, 1 \mathrm{H}), 6.26(\mathrm{~s}, 1 \mathrm{H})$, $4.35(\mathrm{q}, J=7.2 \mathrm{~Hz}, 2 \mathrm{H}), 3.78(\mathrm{~s}, 3 \mathrm{H}), 1.39(\mathrm{t}, J=7.2 \mathrm{~Hz}, 3 \mathrm{H}) ;{ }^{13} \mathrm{C} \mathrm{NMR}\left(150 \mathrm{MHz}, \mathrm{CDCl}_{3}\right) \delta$ 165.7, 137.1, 136.5, 132.1, 126.1, 123.0, 119.8, 115.6, 112.3, 109.1, 102.8, 61.9, 32.9, 14.1; IR (ATR) 3394, 2991, 1685, 1413, 1297, 1227, 794, $639 \mathrm{~cm}^{-1}$; HRMS m/z [M+] calcd for $\mathrm{C}_{14} \mathrm{H}_{14} \mathrm{BrNO}_{3}$ : 323.0157. Found: 323.0158 .

\section{Ethyl (Z)-3-(6-chloro-1-methyl-1H-indol-3-yl)-2-hydroxyacrylate (4j)}

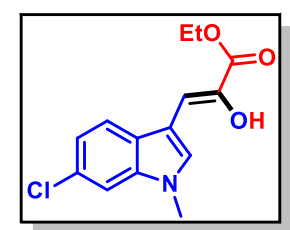

The title compound was prepared according to the general procedure. The product was obtained as a yellow solid. EtOAc:Hex (2:8); Yield: 72\% (100 $\mathrm{mg})$; mp 128-130 C. ${ }^{1} \mathrm{H}$ NMR (600 MHz, $\left.\mathrm{CDCl}_{3}\right) \delta 7.78(\mathrm{~s}, 1 \mathrm{H}), 7.66(\mathrm{~d}, J=$ $8.4 \mathrm{~Hz}, 1 \mathrm{H}), 7.30$ (s, 1H), 7.14 (d, J=8.4 Hz, 1H), 6.83 (s, 1H), 6.29 (s, 1H), $4.35(\mathrm{q}, J=7.2 \mathrm{~Hz}, 2 \mathrm{H}), 3.77(\mathrm{~s}, 3 \mathrm{H}), 1.39(\mathrm{t}, J=7.2 \mathrm{~Hz}, 3 \mathrm{H}) ;{ }^{13} \mathrm{C} \mathrm{NMR}\left(150 \mathrm{MHz}, \mathrm{CDCl}_{3}\right) \delta$ 165.7, 136.6, 136.4, 132.1, 128.0, 125.7, 120.3, 119.4, 109.3, 109.1, 102.9, 61.8, 32.9, 14.1; IR (ATR) 3392, 2920, 1681, 1458, 1376, 1222, 1059, 804, $630 \mathrm{~cm}^{-1}$; HRMS m/z $\left[\mathrm{M}^{+}\right]$calcd for $\mathrm{C}_{14} \mathrm{H}_{14} \mathrm{ClNO}_{3}$ : 279.0662. Found: 279.0663.

\section{Ethyl (Z)-3-(6-fluoro-1-methyl-1H-indol-3-yl)-2-hydroxyacrylate (4k)}

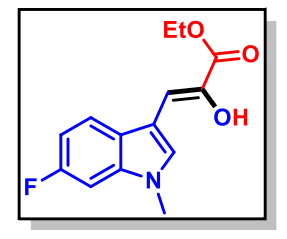

The title compound was prepared according to the general procedure. The product was obtained as a pink solid. EtOAc:Hex (1:9); Yield: 65\% (85 mg); 
mp 94-96 ${ }^{\circ} \mathrm{C} .{ }^{1} \mathrm{H}$ NMR $\left(600 \mathrm{MHz}, \mathrm{CDCl}_{3}\right) \delta 7.78(\mathrm{~s}, 1 \mathrm{H}), 7.67(\mathrm{dd}, J=9.0,5.4 \mathrm{~Hz}, 1 \mathrm{H}), 6.98(\mathrm{dd}$, $J=9.6,2.4 \mathrm{~Hz}, 1 \mathrm{H}), 6.93(\mathrm{td}, J=9.4,2.4 \mathrm{~Hz}, 1 \mathrm{H}), 6.85(\mathrm{~s}, 1 \mathrm{H}), 6.25(\mathrm{~s}, 1 \mathrm{H}), 4.35$ (q, $J=7.2 \mathrm{~Hz}$, $2 \mathrm{H}), 3.76(\mathrm{~s}, 3 \mathrm{H}), 1.39(\mathrm{t}, J=7.2 \mathrm{~Hz}, 3 \mathrm{H}) ;{ }^{13} \mathrm{C} \mathrm{NMR}\left(150 \mathrm{MHz}, \mathrm{CDCl}_{3}\right) \delta 166.0,160.1\left(\mathrm{C}-\mathrm{F},{ }^{1} J_{\mathrm{C}-}\right.$ $\mathrm{F}=240.0 \mathrm{~Hz}), 136.6,136.5\left(\mathrm{C}-\mathrm{F},{ }^{3} J_{\mathrm{C}-\mathrm{F}}=12.0 \mathrm{~Hz}\right), 132.1\left(\mathrm{C}-\mathrm{F},{ }^{4} J_{\mathrm{C}-\mathrm{F}}=3.0 \mathrm{~Hz}\right), 123.8,119.5(\mathrm{C}-\mathrm{F}$, $\left.{ }^{3} J_{\mathrm{C}-\mathrm{F}}=10.2 \mathrm{~Hz}\right), 109.5,108.6\left(\mathrm{C}-\mathrm{F},{ }^{2} J_{\mathrm{C}-\mathrm{F}}=24.3 \mathrm{~Hz}\right), 103.3,95.9\left(\mathrm{C}-\mathrm{F},{ }^{2} J_{\mathrm{C}-\mathrm{F}}=26.1 \mathrm{~Hz}\right), 62.0$, 33.2, 14.3; IR (ATR) 3401, 2923, 1727, 1464, 1228, 924, $814 \mathrm{~cm}^{-1}$; HRMS m/z [M+] calcd for $\mathrm{C}_{14} \mathrm{H}_{14} \mathrm{FNO}_{3}$ : 263.0958. Found: 263.0960.

\section{Ethyl (Z)-3-(1,7-dimethyl-1H-indol-3-yl)-2-hydroxyacrylate (4l)}

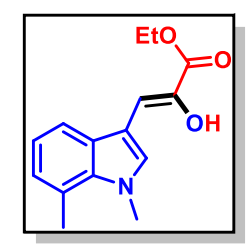

The title compound was prepared according to the general procedure. The product was obtained as a yellow solid. EtOAc:Hex (2:8); Yield: 63\% (82 mg); mp 175-177 ${ }^{\circ} \mathrm{C} .{ }^{1} \mathrm{H}$ NMR $\left(600 \mathrm{MHz}, \mathrm{CDCl}_{3}\right) \delta 7.71(\mathrm{~s}, 1 \mathrm{H}), 7.59$ (d, J=7.8 Hz, 1H), 7.03 (t, $J=7.8 \mathrm{~Hz}, 1 \mathrm{H}), 6.94(\mathrm{~d}, J=7.2 \mathrm{~Hz}, 1 \mathrm{H}), 6.90(\mathrm{~s}, 1 \mathrm{H}), 6.20(\mathrm{~s}, 1 \mathrm{H})$, $4.35(\mathrm{q}, J=6.6 \mathrm{~Hz}, 2 \mathrm{H}), 4.08(\mathrm{~s}, 3 \mathrm{H}), 2.75(\mathrm{~s}, 3 \mathrm{H}), 1.39(\mathrm{t}, J=7.2 \mathrm{~Hz}, 3 \mathrm{H}) ;{ }^{13} \mathrm{C} \mathrm{NMR}(150 \mathrm{MHz}$, $\left.\mathrm{CDCl}_{3}\right) \delta 166.1,136.2,135.2,133.5,128.5,124.9,121.4,120.2,116.6,108.7,103.7,61.9,37.1$, 19.6, 14.3; IR (ATR) 3438, 2981, 1717, 1456, 1226, 1299, 1056, $632 \mathrm{~cm}^{-1}$; HRMS m/z [M+] calcd for $\mathrm{C}_{15} \mathrm{H}_{17} \mathrm{NO}_{3}$ : 259.1208. Found: 259.1205.

\section{Ethyl (Z)-2-hydroxy-3-(1-methyl-1,6,7,8-tetrahydrocyclopenta[g]indol-3-yl)acrylate (4m)}

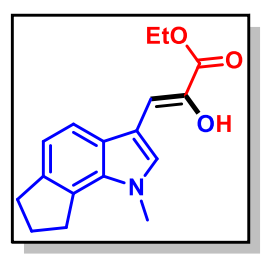

The title compound was prepared according to the genreal procedure. The product was obtained as a pale green solid. EtOAc:Hex (2:8); Yield: 71\% (101 $\mathrm{mg}) ; \mathrm{mp} 153-155^{\circ} \mathrm{C} .{ }^{1} \mathrm{H}$ NMR $\left(600 \mathrm{MHz}, \mathrm{CDCl}_{3}\right) \delta 7.69(\mathrm{~s}, 1 \mathrm{H}), 7.54(\mathrm{~d}, J=$ $7.8 \mathrm{~Hz}, 1 \mathrm{H}), 7.07(\mathrm{~d}, J=8.4 \mathrm{~Hz}, 1 \mathrm{H}), 6.91(\mathrm{~s}, 1 \mathrm{H}), 6.20(\mathrm{~s}, 1 \mathrm{H}), 4.36$ (q, $J=$ $6.6 \mathrm{~Hz}, 2 \mathrm{H}), 3.97(\mathrm{~s}, 3 \mathrm{H}), 3.36(\mathrm{t}, J=7.8 \mathrm{~Hz}, 2 \mathrm{H}), 3.01(\mathrm{t}, J=7.2 \mathrm{~Hz}, 2 \mathrm{H}), 2.18$ (p, J=7.4 Hz, $2 \mathrm{H}), 1.39(\mathrm{t}, J=7.2 \mathrm{~Hz}, 3 \mathrm{H}) ;{ }^{13} \mathrm{C} \mathrm{NMR}\left(150 \mathrm{MHz}, \mathrm{CDCl}_{3}\right) \delta 165.9,139.2,135.8,133.6,131.7$, 126.6, 125.2, 116.9, 116.5, 109.0, 103.9, 61.7, 35.2, 32.4, 31.0, 25.2, 14.1; IR (ATR) 3387, 2933,

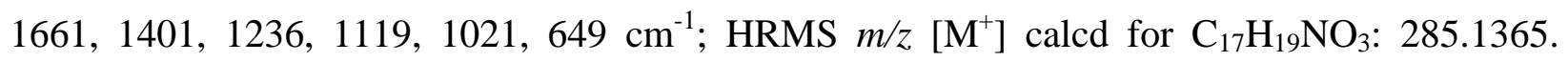
Found: 285.1367. 


\section{Ethyl (Z)-2-hydroxy-3-(1-methyl-1H-benzo[g]indol-3-yl)acrylate (4n)}

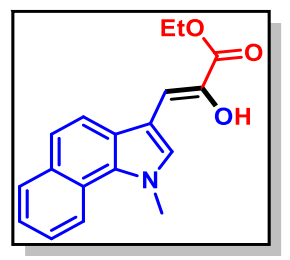

The title compound was prepared according to the general procedure. The product was obtained as a light green solid. EtOAc:Hex (1:9); Yield: 65\% (96 $\mathrm{mg})$; mp 134-136 ${ }^{\circ} \mathrm{C} .{ }^{1} \mathrm{H} \mathrm{NMR}\left(600 \mathrm{MHz}, \mathrm{CDCl}_{3}\right) \delta 8.46(\mathrm{~d}, J=8.4 \mathrm{~Hz}, 1 \mathrm{H})$, $7.95(\mathrm{~d}, J=7.8 \mathrm{~Hz}, 1 \mathrm{H}), 7.85(\mathrm{~d}, J=8.4 \mathrm{~Hz}, 1 \mathrm{H}), 7.83(\mathrm{~s}, 1 \mathrm{H}), 7.57(\mathrm{~d}, J=$ $9.0 \mathrm{~Hz}, 1 \mathrm{H}), 7.54(\mathrm{t}, J=7.2 \mathrm{~Hz}, 1 \mathrm{H}), 7.44(\mathrm{t}, J=8.4 \mathrm{~Hz}, 1 \mathrm{H}), 7.00(\mathrm{~s}, 1 \mathrm{H}), 6.27(\mathrm{~s}, 1 \mathrm{H}), 4.37$ (q, $J=7.2 \mathrm{~Hz}, 2 \mathrm{H}), 4.38(\mathrm{~s}, 3 \mathrm{H}), 1.41(\mathrm{t}, J=7.2 \mathrm{~Hz}, 3 \mathrm{H}) ;{ }^{13} \mathrm{C} \mathrm{NMR}\left(150 \mathrm{MHz}, \mathrm{CDCl}_{3}\right) \delta 165.9$, $136.4,131.7,131.3,129.6,129.0,125.3,124.4,123.4,122.9,121.3,120.3,118.1,109.6,103.4$, 61.8, 38.6, 14.1; IR (ATR) 3402, 2976, 1726, 1386, 1168, 1024, 798, 629, $512 \mathrm{~cm}^{-1}$; HRMS m/z $\left[\mathrm{M}^{+}\right]$calcd for $\mathrm{C}_{18} \mathrm{H}_{17} \mathrm{NO}_{3}$ : 295.1208. Found: 295.1208 .

\section{Ethyl (Z)-2-(1,2-dimethyl-1H-indol-3-yl)-3-(2-ethoxy-2-oxoethoxy)acrylate (6a)}

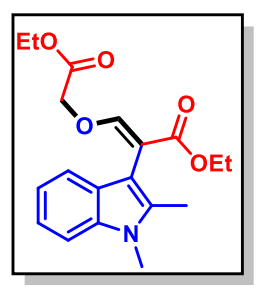

The title compound was prepared according to the general procedure. The product was obtained as a pink solid. EtOAc:Hex (1:9); Yield: 56\% (97 mg); mp 73-75 ${ }^{\circ} \mathrm{C} .{ }^{1} \mathrm{H}$ NMR $\left(600 \mathrm{MHz}, \mathrm{CDCl}_{3}\right) \delta 7.61(\mathrm{~s}, 1 \mathrm{H}), 7.38(\mathrm{~d}, J=8.4 \mathrm{~Hz}$, $1 \mathrm{H}), 7.22(\mathrm{~d}, J=8.4 \mathrm{~Hz}, 1 \mathrm{H}), 7.12(\mathrm{t}, J=8.4 \mathrm{~Hz}, 1 \mathrm{H}), 7.04(\mathrm{t}, J=7.8 \mathrm{~Hz}, 1 \mathrm{H})$, $4.49(\mathrm{~s}, 2 \mathrm{H}), 4.26(\mathrm{q}, J=7.2 \mathrm{~Hz}, 2 \mathrm{H}), 4.21(\mathrm{q}, J=7.2 \mathrm{~Hz}, 2 \mathrm{H}), 3.66(\mathrm{~s}, 3 \mathrm{H}), 2.31(\mathrm{~s}, 3 \mathrm{H}), 1.30(\mathrm{t}$, $J=7.2 \mathrm{~Hz}, 3 \mathrm{H}), 1.24(\mathrm{t}, J=7.2 \mathrm{~Hz}, 3 \mathrm{H}) ;{ }^{13} \mathrm{C} \mathrm{NMR}\left(150 \mathrm{MHz}, \mathrm{CDCl}_{3}\right) \delta 168.1,167.8,157.7$, 136.4, 135.3, 126.9, 120.2, 119.2, 118.8, 108.4, 103.0, 106.2, 69.6, 61.3, 60.1, 29.4, 14.1, 13.8, 11.4; IR (ATR) 2935, 1955, 1847, 1743, 1441, 1378, 1112, 742, $569 \mathrm{~cm}^{-1}$; HRMS m/z [M+] calcd for $\mathrm{C}_{19} \mathrm{H}_{23} \mathrm{NO}_{5}$ : 345.1576. Found: 345.1574.

\section{Isopropyl (Z)-2-(1,2-dimethyl-1H-indol-3-yl)-3-(2-isopropoxy-2-oxoethoxy)acrylate (6b)}

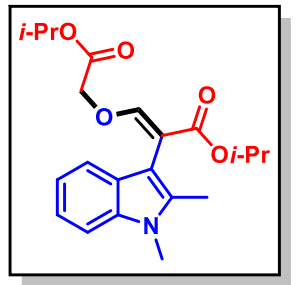

The title compound was prepared according to the general procedure. The product was obtained as a pink solid. EtOAc:Hex (1:9); Yield: 61\% (114 mg); mp 115-117 ${ }^{\circ} \mathrm{C} .{ }^{1} \mathrm{H}$ NMR $\left(600 \mathrm{MHz}, \mathrm{CDCl}_{3}\right) \delta 7.60(\mathrm{~s}, 1 \mathrm{H}), 7.41(\mathrm{~d}, J=7.8$ $\mathrm{Hz}, 1 \mathrm{H}), 7.26(\mathrm{~d}, J=6.0 \mathrm{~Hz}, 1 \mathrm{H}), 7.13(\mathrm{t}, J=7.2 \mathrm{~Hz}, 1 \mathrm{H}), 7.05(\mathrm{t}, J=7.2 \mathrm{~Hz}$, $1 \mathrm{H}), 5.15(\mathrm{q}, J=6.0 \mathrm{~Hz}, 2 \mathrm{H}), 4.48(\mathrm{~s}, 2 \mathrm{H}), 3.69(\mathrm{~s}, 3 \mathrm{H}), 2.33(\mathrm{~s}, 3 \mathrm{H}), 1.30(\mathrm{~d}$, $J=6.0 \mathrm{~Hz}, 6 \mathrm{H}), 1.24(\mathrm{~d}, J=6.0 \mathrm{~Hz}, 6 \mathrm{H}) ;{ }^{13} \mathrm{C} \mathrm{NMR}\left(150 \mathrm{MHz}, \mathrm{CDCl}_{3}\right) \delta 167.6,167.3,157.5$, $136.4,135.3,126.9,120.1,119.4,118.7,108.3,106.5,103.2,69.8,69.3,67.3,29.3,21.7,21.5$, 
11.5; IR (ATR) 2979, 1848, 1752, 1693, 1268, 1097, 747, $434 \mathrm{~cm}^{-1}$; HRMS $\mathrm{m} / z\left[\mathrm{M}^{+}\right]$calcd for $\mathrm{C}_{21} \mathrm{H}_{27} \mathrm{NO}_{5}: 373.1889$. Found: 373.1892 .

\section{Ethyl (Z)-2-(1-benzyl-2-methyl-1H-indol-3-yl)-3-(2-ethoxy-2-oxoethoxy)acrylate (6c)}

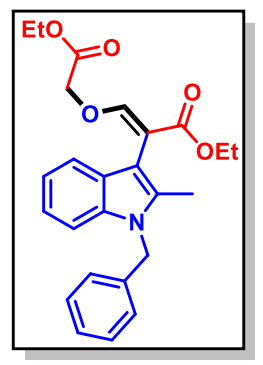

The title compound was prepared according to the general procedure. The product was obtained as a pink solid. EtOAc:Hex (1:9); Yield: 58\% (122 mg); mp 110-112 ${ }^{\circ} \mathrm{C} .{ }^{1} \mathrm{H}$ NMR $\left(600 \mathrm{MHz}, \mathrm{CDCl}_{3}\right) \delta 7.63(\mathrm{~s}, 1 \mathrm{H}), 7.44(\mathrm{~d}, J=6.6 \mathrm{~Hz}$, $1 \mathrm{H}), 7.25-7.23(\mathrm{~m}, 2 \mathrm{H}), 7.20(\mathrm{~d}, J=7.2 \mathrm{~Hz}, 1 \mathrm{H}), 7.17(\mathrm{~d}, J=7.8 \mathrm{~Hz}, 1 \mathrm{H})$, 7.07-7.03 (m, 2H), 6.99 (d, $J=7.8 \mathrm{~Hz}, 2 \mathrm{H}), 5.32(\mathrm{~s}, 2 \mathrm{H}), 4.49$ (s, 2H), $4.25(\mathrm{q}$, $J=6.0 \mathrm{~Hz}, 2 \mathrm{H}), 4.20(\mathrm{q}, J=7.2 \mathrm{~Hz}, 2 \mathrm{H}), 2.23(\mathrm{~s}, 3 \mathrm{H}), 1.28(\mathrm{t}, J=7.2 \mathrm{~Hz}, 3 \mathrm{H}), 1.22(\mathrm{t}, J=7.2$ $\mathrm{Hz}, 3 \mathrm{H}) ;{ }^{13} \mathrm{C} \mathrm{NMR}\left(150 \mathrm{MHz}, \mathrm{CDCl}_{3}\right) \delta 168.1,157.8,137.6,136.2,135.3,128.4,127.3,126.9$, 125.8, 120.5, 119.4, 119.1, 108.8, 69.6, 61.3, 60.1, 46.4, 14.1, 13.9, 11.4; IR (ATR) 2925, 1686, $1440,1266,1137,1024,733,448 \mathrm{~cm}^{-1}$; HRMS $m / z\left[\mathrm{M}^{+}\right]$calcd for $\mathrm{C}_{25} \mathrm{H}_{27} \mathrm{NO}_{5}: 421.1889$. Found: 421.1892 .

\section{Isopropyl (Z)-2-(2-ethyl-1-methyl-1H-indol-3-yl)-3-(2-isopropoxy-2-oxoethoxy)acrylate (6d)}

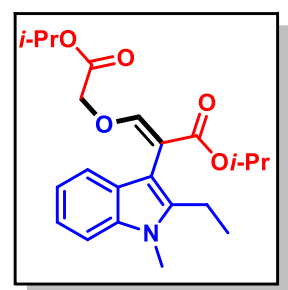

The title compound was prepared according to the general procedure. The product was obtained as a white solid. EtOAc:Hex (1:9); Yield: 52\% (101 $\mathrm{mg}$ ); mp 123-125 ${ }^{\circ} \mathrm{C} .{ }^{1} \mathrm{H}$ NMR (600 MHz, $\left.\mathrm{CDCl}_{3}\right) \delta 7.58$ (s, $\left.1 \mathrm{H}\right), 7.35$ (d, $J=$ $7.8 \mathrm{~Hz}, 1 \mathrm{H}), 7.22(\mathrm{~d}, J=8.4 \mathrm{~Hz}, 1 \mathrm{H}), 7.11(\mathrm{t}, J=7.2 \mathrm{~Hz}, 1 \mathrm{H}), 7.02(\mathrm{t}, J=$ $7.2 \mathrm{~Hz}, 1 \mathrm{H}), 5.11-5.07(\mathrm{~m}, 2 \mathrm{H}), 4.43(\mathrm{~s}, 2 \mathrm{H}), 3.70(\mathrm{~s}, 3 \mathrm{H}), 2.74(\mathrm{q}, J=7.2$ $\mathrm{Hz}, 2 \mathrm{H}), 1.27(\mathrm{~d}, J=6.0 \mathrm{~Hz}, 6 \mathrm{H}), 1.18(\mathrm{~d}, J=4.8 \mathrm{~Hz}, 6 \mathrm{H}), 1.19(\mathrm{~s}, 3 \mathrm{H}) ;{ }^{13} \mathrm{C}$ NMR $(150 \mathrm{MHz}$, $\left.\mathrm{CDCl}_{3}\right) \delta 167.6,167.4,158.1,140.4,136.6,120.3,119.2,118.8,108.4,106.3,69.7,69.3,67.2$, 29.5, 21.6, 21.5, 18.5, 13.5; IR (ATR) 2929, 1841, 1633, 1695, 1267, 1027, 741, $424 \mathrm{~cm}^{-1}$; HRMS $m / z\left[\mathrm{M}^{+}\right]$calcd for $\mathrm{C}_{22} \mathrm{H}_{29} \mathrm{NO}_{5}: 387.2046$. Found: 387.2044 .

Isopropyl (Z)-3-(2-isopropoxy-2-oxoethoxy)-2-(1-methyl-2-phenyl-1H-indol-3-yl)acrylate $(6 e)$

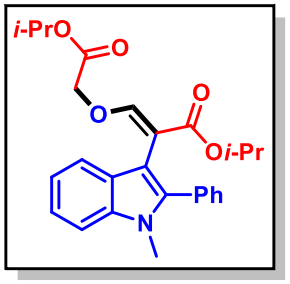

The title compound was prepared according to the general procedure. The product was obtained as a yellow solid. EtOAc:Hex (1:9); Yield: 66\% (144 
mg); mp 89-91 ${ }^{\circ} \mathrm{C} .{ }^{1} \mathrm{H}$ NMR $\left(600 \mathrm{MHz}, \mathrm{CDCl}_{3}\right) \delta 7.56(\mathrm{~d}, J=7.8 \mathrm{~Hz}, 1 \mathrm{H}), 7.51(\mathrm{~s}, 1 \mathrm{H}), 7.436-$ $7.386(\mathrm{~m}, 4 \mathrm{H}), 7.33$ (t, $J=7.8 \mathrm{~Hz}, 2 \mathrm{H}), 7.22(\mathrm{t}, J=7.8 \mathrm{~Hz}, 1 \mathrm{H}), 7.12(\mathrm{t}, J=7.8 \mathrm{~Hz}, 1 \mathrm{H}), 5.11$ (p, $J=6.6 \mathrm{~Hz}, 1 \mathrm{H}), 4.78(\mathrm{p}, J=6.6 \mathrm{~Hz}, 1 \mathrm{H}), 4.37(\mathrm{~s}, 2 \mathrm{H}), 3.69(\mathrm{~s}, 3 \mathrm{H}), 1.27(\mathrm{~d}, J=6.0 \mathrm{~Hz}, 6 \mathrm{H})$, $0.91(\mathrm{~d}, J=6.6 \mathrm{~Hz}, 6 \mathrm{H}) ;{ }^{13} \mathrm{C} \mathrm{NMR}\left(150 \mathrm{MHz}, \mathrm{CDCl}_{3}\right) \delta 167.5,167.1,158.2,139.0,137.1$, 132.3, 130.0, 127.9, 127.4, 127.1, 121.3, 120.3, 119.3, 109.2, 106.6, 105.0, 69.7, 69.2, 67.1, 31.0, 21.5, 21.2; IR (ATR) 2974, 1848, 1738, 1462, 1373, 1096, 741, $579 \mathrm{~cm}^{-1} ; \mathrm{HRMS} \mathrm{m} / \mathrm{z}\left[\mathrm{M}^{+}\right]$ calcd for $\mathrm{C}_{26} \mathrm{H}_{29} \mathrm{NO}_{5}$ : 435.2046. Found: 435.2049.

\section{Ethyl (Z)-3-hydroxy-2-(1-methyl-1H-indol-3-yl)acrylate (7a)}

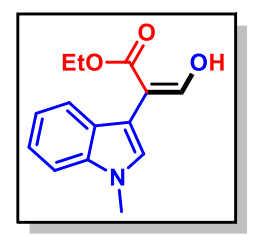

The title compound was prepared according to the general procedure. The product was obtained as a pale yellow solid. EtOAc:Hex (1:9); Yield: $72 \%(88$ $\mathrm{mg})$; mp 106-108 ${ }^{\circ} \mathrm{C} .{ }^{1} \mathrm{H}$ NMR $\left(600 \mathrm{MHz}, \mathrm{CDCl}_{3}\right) \delta 12.10(\mathrm{~d}, J=12.0 \mathrm{~Hz}, 1 \mathrm{H})$, $7.53(\mathrm{~d}, J=7.8 \mathrm{~Hz}, 1 \mathrm{H}), 7.38(\mathrm{~d}, J=12.0 \mathrm{~Hz}, 1 \mathrm{H}), 7.31(\mathrm{~d}, J=7.8 \mathrm{~Hz}, 1 \mathrm{H}), 7.22(\mathrm{~d}, J=7.2 \mathrm{~Hz}$, 1H), $7.11(\mathrm{t}, J=7.8 \mathrm{~Hz}, 1 \mathrm{H}), 6.96(\mathrm{~s}, 1 \mathrm{H}), 4.28(\mathrm{q}, J=7.2 \mathrm{~Hz}, 2 \mathrm{H}), 3.78(\mathrm{~s}, 3 \mathrm{H}), 1.26(\mathrm{t}, J=7.2$ $\mathrm{Hz}, 3 \mathrm{H}) ;{ }^{13} \mathrm{C} \mathrm{NMR}\left(150 \mathrm{MHz}, \mathrm{CDCl}_{3}\right) \delta 172.1,162.5,136.5,127.6,127.4,121.5,119.6,119.1$, 109.1, 107.6, 100.4, 60.5, 32.5, 14.0; IR (ATR) 3461, 2932, 1718, 1470, 1373, 1236, 1167, 1027, $745,556 \mathrm{~cm}^{-1}$; HRMS $\mathrm{m} / z\left[\mathrm{M}^{+}\right]$calcd for $\mathrm{C}_{14} \mathrm{H}_{15} \mathrm{NO}_{3}$ : 245.1052. Found: 245.1051.

\section{Ethyl (Z)-3-hydroxy-2-(1-methyl-2-phenyl-1H-indol-3-yl)acrylate (7b)}

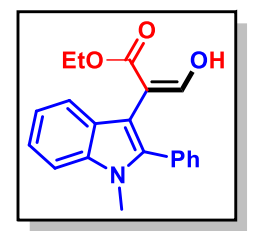

The title compound was prepared according to the general procedure. The product was obtained as a yellow solid. EtOAc:Hex (1:9); Yield: 67\% (108 mg); mp 101-103 ${ }^{\circ} \mathrm{C} .{ }^{1} \mathrm{H}$ NMR $\left(600 \mathrm{MHz}, \mathrm{CDCl}_{3}\right) \delta 12.11(\mathrm{~d}, J=12.6 \mathrm{~Hz}, 1 \mathrm{H}), 7.52$ (d, $J=7.8 \mathrm{~Hz}, 1 \mathrm{H}), 7.43(\mathrm{t}, J=7.2 \mathrm{~Hz}, 2 \mathrm{H}), 7.38-7.34(\mathrm{~m}, 4 \mathrm{H}), 7.27$ (t, $J=7.8$ $\mathrm{Hz}, 1 \mathrm{H}), 7.18$ (s, 1H), 7.15 (d, $J=7.2 \mathrm{~Hz}, 1 \mathrm{H}), 4.04$ (q, $J=7.2 \mathrm{~Hz}, 2 \mathrm{H}), 3.67$ (s, 3H), 1.01 (t, $J=$ $7.2 \mathrm{~Hz}, 3 \mathrm{H}) ;{ }^{13} \mathrm{C}$ NMR $\left(150 \mathrm{MHz}, \mathrm{CDCl}_{3}\right) \delta 172.4,164.8,139.4,136.9,131.9,130.4,128.3$, 127.9, 121.9, 119.8, 119.3, 109.4, 106.2, 99.8, 60.4, 31.1, 13.9; IR (ATR) 2920, 2851, 1705, 1664, 1605, 1467, 1351, 1105, 856, 696, $455 \mathrm{~cm}^{-1}$; HRMS $\mathrm{m} / z\left[\mathrm{M}^{+}\right]$calcd for $\mathrm{C}_{20} \mathrm{H}_{19} \mathrm{NO}_{3}$ : 321.1365. Found: 321.1367. 


\section{Isopropyl (Z)-3-hydroxy-2-(1-methyl-1H-indol-3-yl)acrylate (7c)}

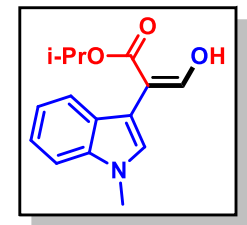

The title compound was prepared according to the general procedure. The product was obtained as a pink solid. EtOAc:Hex (1:9); Yield: 62\% (80 mg); mp 103-105 ${ }^{\circ} \mathrm{C} .{ }^{1} \mathrm{H}$ NMR $\left(600 \mathrm{MHz}, \mathrm{CDCl}_{3}\right) \delta 12.18(\mathrm{~d}, J=12.6 \mathrm{~Hz}, 1 \mathrm{H}), 7.54(\mathrm{~d}, J$ $=7.8 \mathrm{~Hz}, 1 \mathrm{H}), 7.38(\mathrm{~d}, J=12.6 \mathrm{~Hz}, 1 \mathrm{H}), 7.30(\mathrm{~d}, J=8.4 \mathrm{~Hz}, 1 \mathrm{H}), 7.22(\mathrm{t}, J=$

$7.8 \mathrm{~Hz}, 1 \mathrm{H}), 7.10$ (t, $J=7.8 \mathrm{~Hz}, 1 \mathrm{H}), 6.94(\mathrm{~s}, 1 \mathrm{H}), 5.20(\mathrm{p}, J=6.0 \mathrm{~Hz}, 1 \mathrm{H}), 3.77$ (s, 3H), 1.24 $(\mathrm{d}, J=6.0 \mathrm{~Hz}, 6 \mathrm{H}) ;{ }^{13} \mathrm{C} \mathrm{NMR}\left(150 \mathrm{MHz}, \mathrm{CDCl}_{3}\right) \delta 171.6,162.3,136.5,127.5,127.3,121.4$, 119.8, 118.9, 109.0, 107.8, 100.8, 68.3, 32.5, 21.5; IR (ATR) 3059, 2930, 1387, 1273, 1167, 1095, 943, 814, 739, $419 \mathrm{~cm}^{-1}$; HRMS m/z $\left[\mathrm{M}^{+}\right]$calcd for $\mathrm{C}_{15} \mathrm{H}_{17} \mathrm{NO}_{3}$ : 259.1208. Found: 259.1210 .

\section{Cyclohexyl (Z)-3-hydroxy-2-(1-methyl-1H-indol-3-yl)acrylate (7d)}

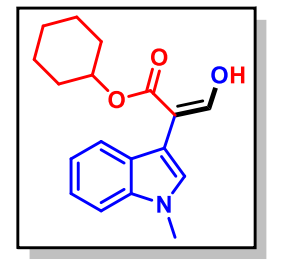

The title compound was prepared according to the general procedure. The product was obtained as a pale yellow solid. EtOAc:Hex (1:9); Yield: 57\% (85 $\mathrm{mg}) ; \mathrm{mp}$ 120-122 ${ }^{\circ} \mathrm{C} .{ }^{1} \mathrm{H} \mathrm{NMR}\left(600 \mathrm{MHz}, \mathrm{CDCl}_{3}\right) \delta 12.17(\mathrm{~d}, J=12.6 \mathrm{~Hz}, 1 \mathrm{H})$, $7.55(\mathrm{~d}, J=7.8 \mathrm{~Hz}, 1 \mathrm{H}), 7.39(\mathrm{~d}, J=12.0 \mathrm{~Hz}, 1 \mathrm{H}), 7.29(\mathrm{~d}, J=8.4 \mathrm{~Hz}, 1 \mathrm{H})$, $7.21(\mathrm{t}, J=7.2 \mathrm{~Hz}, 1 \mathrm{H}), 7.09(\mathrm{t}, J=7.8 \mathrm{~Hz}, 1 \mathrm{H}), 6.95(\mathrm{~s}, 1 \mathrm{H}), 4.94(\mathrm{dt}, J=9.0,4.8 \mathrm{~Hz}, 1 \mathrm{H}), 3.77$ (s, 3H), 1.90-1.83 (m, 2H), 1.66-1.59 (m, 2H), 1.51-1.46 (m, 1H), 1.43-1.37 (m, 2H), 1.36-1.30 $(\mathrm{m}, 2 \mathrm{H}), 1.19-1.13(\mathrm{~m}, 1 \mathrm{H}) ;{ }^{13} \mathrm{C} \mathrm{NMR}\left(150 \mathrm{MHz}, \mathrm{CDCl}_{3}\right) \delta 171.5,162.2,136.5,127.6,121.3$, 119.8, 118.9, 109.0, 107.8, 100.9, 73.2, 32.5, 31.2, 25.0, 23.4; IR (ATR) 2934, 2859, 1723, 1653, 1324, 1157, 1016, 809, 735, $646 \mathrm{~cm}^{-1}$; HRMS m/z $\left[\mathrm{M}^{+}\right]$calcd for $\mathrm{C}_{18} \mathrm{H}_{21} \mathrm{NO}_{3}$ : 299.1521. Found: 299.1519.

\section{Ethyl (Z)-2-(1-benzyl-1H-indol-3-yl)-3-hydroxyacrylate (7e)}

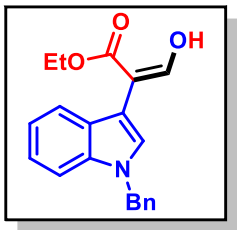

The title compound was prepared according to the general procedure. The product was obtained as a white solid. EtOAc:Hex (1:9); Yield: 67\% (107 mg); mp 106-108 ${ }^{\circ} \mathrm{C} .{ }^{1} \mathrm{H}$ NMR $\left(600 \mathrm{MHz}, \mathrm{CDCl}_{3}\right) \delta 12.12(\mathrm{~d}, J=12.6 \mathrm{~Hz}, 1 \mathrm{H}), 7.58$ $(\mathrm{d}, J=7.8 \mathrm{~Hz}, 1 \mathrm{H}), 7.43(\mathrm{~d}, J=12.6 \mathrm{~Hz}, 1 \mathrm{H}), 7.33-7.26(\mathrm{~m}, 4 \mathrm{H}), 7.19$ (t, $J=7.8$ $\mathrm{Hz}, 1 \mathrm{H}), 7.15(\mathrm{~d}, J=7.2 \mathrm{~Hz}, 2 \mathrm{H}), 7.14(\mathrm{t}, J=7.8 \mathrm{~Hz}, 1 \mathrm{H}), 7.05(\mathrm{~s}, 1 \mathrm{H}), 5.30(\mathrm{~s}, 2 \mathrm{H}), 4.28(\mathrm{q}, J$ $=6.6 \mathrm{~Hz}, 2 \mathrm{H}), 1.25(\mathrm{t}, J=7.2 \mathrm{~Hz}, 3 \mathrm{H}) ;{ }^{13} \mathrm{C} \mathrm{NMR}\left(150 \mathrm{MHz}, \mathrm{CDCl}_{3}\right) \delta 172.0,162.6,137.0$, 
136.1, 128.5, 127.6, 127.4, 127.1, 126.7, 121.6, 119.7, 119.3, 109.5, 108.2, 100.3, 60.5, 49.7, 13.9; IR (ATR) 3052, 2980, 1747, 1657, 1460, 1385, 1161, 946, $731 \mathrm{~cm}^{-1}$; HRMS m/z $\left[\mathrm{M}^{+}\right]$ calcd for $\mathrm{C}_{20} \mathrm{H}_{19} \mathrm{NO}_{3}$ : 321.1365. Found: 321.1366 .

\section{Ethyl (Z)-2-(5-bromo-1-methyl-1H-indol-3-yl)-3-hydroxyacrylate (7f)}

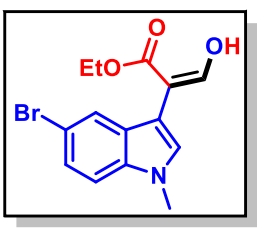

The title compound was prepared according to the general procedure. The product was obtained as a light brown solid. EtOAc:Hex (2:8); Yield: 60\% (97 $\mathrm{mg})$; mp 92-94 ${ }^{\circ} \mathrm{C} .{ }^{1} \mathrm{H} \mathrm{NMR}\left(600 \mathrm{MHz}, \mathrm{CDCl}_{3}\right) \delta 12.08(\mathrm{~d}, J=12.6 \mathrm{~Hz}, 1 \mathrm{H})$, $7.64(\mathrm{~s}, 1 \mathrm{H}), 7.33(\mathrm{~d}, J=12.6 \mathrm{~Hz}, 1 \mathrm{H}), 7.29(\mathrm{~d}, J=9.0 \mathrm{~Hz}, 1 \mathrm{H}), 7.16(\mathrm{~d}, J=$ $9.0 \mathrm{~Hz}, 1 \mathrm{H}), 6.94(\mathrm{~s}, 1 \mathrm{H}), 4.28(\mathrm{q}, J=6.6 \mathrm{~Hz}, 2 \mathrm{H}), 3.75(\mathrm{~s}, 3 \mathrm{H}), 1.26(\mathrm{~d}, J=7.2 \mathrm{~Hz}, 3 \mathrm{H}) ;{ }^{13} \mathrm{C}$ NMR $\left(150 \mathrm{MHz}, \mathrm{CDCl}_{3}\right) \delta 171.9,162.8,135.4,129.3,128.8,124.5,122.6,112.7,110.8,107.6$, 100.1, 60.9, 32.9, 14.1; IR (ATR) 3107, 2977, 1724, 1469, 1264, 1164, 941, 790, $578 \mathrm{~cm}^{-1}$; HRMS $m / z\left[\mathrm{M}^{+}\right]$calcd for $\mathrm{C}_{14} \mathrm{H}_{14} \mathrm{BrNO}_{3}$ : 323.0157. Found: 323.0160 .

\section{Ethyl (Z)-3-hydroxy-2-(1-methyl-1,6,7,8-tetrahydrocyclopenta[g]indol-3-yl)acrylate (7g)}

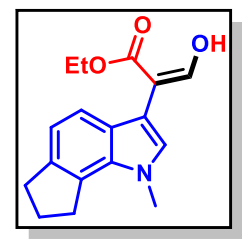

The title compound was prepared according to the general procedure. The product was obtained as a pale yellow solid. EtOAc:Hex (2:8); Yield: 43\% (61 $\mathrm{mg})$; mp 100-102 ${ }^{\circ} \mathrm{C} .{ }^{1} \mathrm{H}$ NMR $\left(600 \mathrm{MHz}, \mathrm{CDCl}_{3}\right) \delta 12.07(\mathrm{~d}, J=12.6 \mathrm{~Hz}, 1 \mathrm{H})$, $7.33(\mathrm{~d}, J=12.6 \mathrm{~Hz}, 1 \mathrm{H}), 7.29$ (d, $J=7.8 \mathrm{~Hz}, 1 \mathrm{H}), 6.99$ (d, $J=7.8 \mathrm{~Hz}, 1 \mathrm{H}), 6.80$ (s, 1H), 4.26 (q, $J=7.2 \mathrm{~Hz}, 2 \mathrm{H}), 3.93(\mathrm{~s}, 3 \mathrm{H}), 3.36(\mathrm{t}, J=7.2 \mathrm{~Hz}, 2 \mathrm{H}), 2.99$ (t, $J=7.2 \mathrm{~Hz}, 2 \mathrm{H})$, $2.17(\mathrm{p}, J=7.8 \mathrm{~Hz}, 2 \mathrm{H}), 1.25(\mathrm{~d}, J=7.2 \mathrm{~Hz}, 3 \mathrm{H}) ;{ }^{13} \mathrm{C} \mathrm{NMR}\left(150 \mathrm{MHz}, \mathrm{CDCl}_{3}\right) \delta 172.1,162.5$, 138.6, 133.8, 127.6, 126.9, 125.1, 117.9, 116.3, 107.8, 100.5, 60.5, 34.8, 32.4, 31.1, 25.2, 14.1; IR (ATR) 3216, 2924, 1712, 1658, 1394, 1271, 1154, 1021, 807, $581 \mathrm{~cm}^{-1}$; HRMS m/z [M+] calcd for $\mathrm{C}_{17} \mathrm{H}_{19} \mathrm{NO}_{3}$ : 285.1365. Found: 285.1365.

\section{Ethyl (Z)-2-hydroxy-3-(1-methyl-1H-indol-3-yl)acrylate (3aD)}

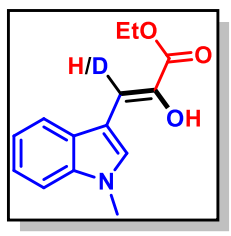

The title compound was prepared according to the general procedure. The product was obtained as a white solid. EtOAc:Hex (1:9); Yield: 64\% (79 mg); mp 96-98 ${ }^{\circ}$ C. ${ }^{1} \mathrm{H}$ NMR (600 MHz, $\left.\mathrm{CDCl}_{3}\right) \delta 7.83(\mathrm{~s}, 1 \mathrm{H}), 7.77$ (d, $J=7.8 \mathrm{~Hz}$, $1 \mathrm{H}), 7.32(\mathrm{~d}, J=7.8 \mathrm{~Hz}, 1 \mathrm{H}), 7.26(\mathrm{t}, J=7.2 \mathrm{~Hz}, 1 \mathrm{H}), 7.19(\mathrm{t}, J=7.8 \mathrm{~Hz}, 1 \mathrm{H})$, $6.94(\mathrm{~s}, 0.5 \mathrm{H}), 6.23(\mathrm{~s}, 1 \mathrm{H}), 4.36(\mathrm{q}, J=7.2 \mathrm{~Hz}, 2 \mathrm{H}), 3.82(\mathrm{~s}, 3 \mathrm{H}), 1.40(\mathrm{t}, J=7.2 \mathrm{~Hz}, 3 \mathrm{H}) ;{ }^{13} \mathrm{C}$ 
NMR $\left(150 \mathrm{MHz}, \mathrm{CDCl}_{3}\right) \delta 165.9,136.3,136.0,131.7,127.3,122.0,119.8,118.4,109.2,108.9$, 103.6, 61.7, 32.9, 14.1; IR (ATR) 3403, 2940, 1663, 1324, 1230, 731, 608,506 $\mathrm{cm}^{-1} ; \mathrm{HRMS} \mathrm{m/z}$ $\left[\mathrm{M}^{+}\right]$calcd for $\mathrm{C}_{14} \mathrm{H}_{14} \mathrm{DNO}_{3}: 246.1115$. Found: 246.1112 .

\section{Ethyl (Z)-3-hydroxy-2-(1-methyl-1H-indol-3-yl)acrylate-d (7aD)}

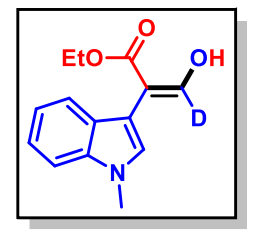

The title compound was prepared according to the general procedure. The product was obtained as a pink solid. EtOAc:Hex (1:9); Yield: $70 \%$ (86 mg); mp 62-64 ${ }^{\circ} \mathrm{C} .{ }^{1} \mathrm{H}$ NMR $\left(600 \mathrm{MHz}, \mathrm{CDCl}_{3}\right) \delta 12.04(\mathrm{~s}, 1 \mathrm{H}), 7.48(\mathrm{~d}, J=7.8 \mathrm{~Hz}, 1 \mathrm{H})$, $7.25(\mathrm{~d}, J=7.8 \mathrm{~Hz}, 1 \mathrm{H}), 7.18(\mathrm{t}, J=8.4 \mathrm{~Hz}, 1 \mathrm{H}), 7.06(\mathrm{t}, J=7.8 \mathrm{~Hz}, 1 \mathrm{H}), 6.91$ $(\mathrm{s}, 1 \mathrm{H}), 4.23(\mathrm{q}, J=7.2 \mathrm{~Hz}, 2 \mathrm{H}), 3.72(\mathrm{~s}, 3 \mathrm{H}), 1.21(\mathrm{t}, J=6.6 \mathrm{~Hz}, 3 \mathrm{H}) ;{ }^{13} \mathrm{C} \mathrm{NMR}(150 \mathrm{MHz}$, $\left.\mathrm{CDCl}_{3}\right) \delta 172.2,162.3\left(\mathrm{C}-\mathrm{D}, \mathrm{t}, J_{\mathrm{C}-\mathrm{D}}=28.3 \mathrm{~Hz}\right), 136.7,127.8,127.6,121.7,119.8,119.2,109.3$, 107.8, 100.5, 60.7, 32.7, 14.2; IR (ATR) 3048, 2987, 1652, 1537, 1475, 1304, 1179, 1020, 816, $741,428 \mathrm{~cm}^{-1}$; HRMS $m / z\left[\mathrm{MH}^{+}\right]$calcd for $\mathrm{C}_{14} \mathrm{H}_{15} \mathrm{DNO}_{3}: 247.1187$. Found: 247.1184 .

\section{Ethyl 3-oxo-3-phenylpropanoate (B) and Ethyl (Z)-3-hydroxy-2-phenylacrylate (A)}

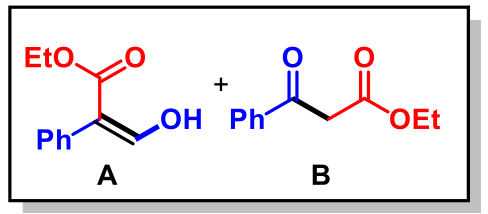

The title compound was prepared according to the general procedure. The product was obtained as a colourless oil. EtOAc:Hex (1:9); Yield: 65\% (62 mg); Characterization data of major keto-form of B. ${ }^{1} \mathrm{H}$ NMR $\left(600 \mathrm{MHz}, \mathrm{CDCl}_{3}\right) \delta 7.93-7.91$ (m, 2H), 7.58-7.53 (m, 1H), 7.48-7.43 (m, 1H), 7.40-7.36 (m, 2H), 4.21-4.17 (m, 2H), 3.96 (s, $2 \mathrm{H}), 1.23(\mathrm{t}, J=7.2 \mathrm{~Hz}, 3 \mathrm{H}) ;{ }^{13} \mathrm{C} \mathrm{NMR}\left(150 \mathrm{MHz}, \mathrm{CDCl}_{3}\right) \delta 192.4,167.4,135.9,133.7,128.7$, 128.4, 61.4, 45.9, 14.0. Characterization data of A. ${ }^{1} \mathrm{H}$ NMR $\left(600 \mathrm{MHz}, \mathrm{CDCl}_{3}\right) \delta 12.10(\mathrm{~d}, J=$ 12.6 Hz, 1H), 7.34-7.25 (6H, m), 4.27-4.22 (m, 2H), 1.29-1.25 (m, 3H); ${ }^{13} \mathrm{C}$ NMR (150 MHz, $\left.\mathrm{CDCl}_{3}\right) \delta 171.4,163.3,133.4,129.3,128.1,126.5,105.4,60.8,14.2 ; \mathrm{HRMS} \mathrm{m} / z\left[\mathrm{MNa}^{+}\right]$calcd for $\mathrm{C}_{11} \mathrm{H}_{12} \mathrm{O}_{3} \mathrm{Na}: 215.0679$. Found: 215.0675.

\section{(Z)-3-Ethoxy-1-(1-methyl-1H-indol-3-yl)-3-oxoprop-1-en-2-yl benzoate (20)}

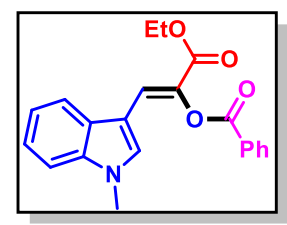

The title compound was prepared according to the general procedure. The product was obtained as a white solid. EtOAc:Hex (2:8); Yield: 94\% (82 mg); mp 159-161 ${ }^{\circ} \mathrm{C} .{ }^{1} \mathrm{H}$ NMR $\left(600 \mathrm{MHz}, \mathrm{CDCl}_{3}\right) \delta 8.27(\mathrm{~d}, J=7.8 \mathrm{~Hz}, 2 \mathrm{H}), 7.81$ $(\mathrm{d}, J=7.2 \mathrm{~Hz}, 1 \mathrm{H}), 7.80(\mathrm{~s}, 1 \mathrm{H}), 7.67(\mathrm{t}, J=7.2 \mathrm{~Hz}, 1 \mathrm{H}), 7.55$ (t, $J=7.8 \mathrm{~Hz}, 2 \mathrm{H}), 7.46(\mathrm{~s}, 1 \mathrm{H})$, 
7.28-7.25 (m, 2H), 7.22 (t, $J=7.2 \mathrm{~Hz}, 1 \mathrm{H}), 4.31(\mathrm{q}, J=7.2 \mathrm{~Hz}, 2 \mathrm{H}), 3.69(\mathrm{~s}, 3 \mathrm{H}), 1.32$ (t, $J=7.2$ $\mathrm{Hz}, 3 \mathrm{H}) .{ }^{13} \mathrm{C}$ NMR $\left(150 \mathrm{MHz}, \mathrm{CDCl}_{3}\right) \delta 164.1,162.6,136.2,133.5,133.2,131.1,130.1,129.0$, 128.5, 127.5, 122.5, 120.7, 119.7, 118.5, 109.4, 107.5, 61.1, 33.1, 14.1 ; IR (ATR) 2978, 1706, $1457,1245,1069,748,693 \mathrm{~cm}^{-1}$; HRMS $\mathrm{m} / z\left[\mathrm{M}^{+}\right]$calcd for $\mathrm{C}_{21} \mathrm{H}_{19} \mathrm{NO}_{4}$ : 349.1314. Found: 349.1317.

Ethyl (Z)-2-(allyloxy)-3-(1-methyl-1H-indol-3-yl)acrylate (21)

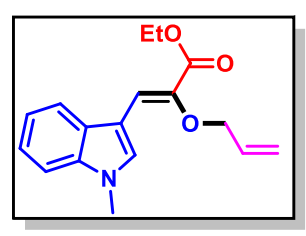

The title compound was prepared according to the general procedure. The product was obtained as a pale yellow solid. EtOAc:Hex (2:8); Yield: $89 \%$ (63 mg); mp 145-147 ${ }^{\circ} \mathrm{C} .{ }^{1} \mathrm{H}$ NMR (600 MHz, $\left.\mathrm{CDCl}_{3}\right) \delta 7.88(\mathrm{~s}, 1 \mathrm{H}), 7.78$ $(\mathrm{d}, J=7.8 \mathrm{~Hz}, 1 \mathrm{H}), 7.45(\mathrm{~s}, 1 \mathrm{H}), 7.32(\mathrm{~d}, J=8.4 \mathrm{~Hz}, 1 \mathrm{H}), 7.26(\mathrm{t}, J=7.2$ $\mathrm{Hz}, 1 \mathrm{H}), 7.20(\mathrm{t}, J=6.6 \mathrm{~Hz}, 1 \mathrm{H}), 6.13-6.05(\mathrm{~m}, 1 \mathrm{H}), 5.39$ (d, $J=17.4 \mathrm{~Hz}, 1 \mathrm{H}), 5.23(\mathrm{~d}, J=10.2$ $\mathrm{Hz}, 1 \mathrm{H}), 4.52(\mathrm{~d}, J=6.0 \mathrm{~Hz}, 2 \mathrm{H}), 4.30(\mathrm{q}, J=7.2 \mathrm{~Hz}, 2 \mathrm{H}), 3.81(\mathrm{~s}, 3 \mathrm{H}), 1.37(\mathrm{t}, J=7.2 \mathrm{~Hz}, 3 \mathrm{H})$; ${ }^{13} \mathrm{C}$ NMR $\left(150 \mathrm{MHz}, \mathrm{CDCl}_{3}\right) \delta 164.5,140.1,136.2,134.1,131.8,127.5,122.1,120.2,118.5$, 117.6, 117.3, 109.2, 108.4, 71.6, 60.4, 33.0, 14.2; IR (ATR) 2931, 1721, 1612, 1459, 1369, 1249, $1016,743 \mathrm{~cm}^{-1} ; \mathrm{HRMS} m / z\left[\mathrm{M}^{+}\right]$calcd for $\mathrm{C}_{17} \mathrm{H}_{19} \mathrm{NO}_{3}: 285.1365$. Found: 285.1367.

\section{Triethyl (4S,5S)-4,5-dihydro-1H-pyrazole-3,4,5-tricarboxylate (2a')}

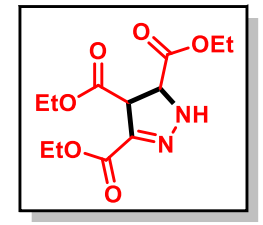

The title compound was prepared according to the general procedure. The product was obtained as a white crystalline solid. EtOAc:Hex (3:7); Yield: 57\% $(82 \mathrm{mg}) ; \mathrm{mp} 93-95^{\circ} \mathrm{C} .{ }^{1} \mathrm{H} \mathrm{NMR}\left(600 \mathrm{MHz}, \mathrm{CDCl}_{3}\right) \delta 6.41(\mathrm{~s}, 1 \mathrm{H}), 4.67$ (d, $J=$ $6.0 \mathrm{~Hz}, 1 \mathrm{H}), 4.31(\mathrm{~d}, J=6.0 \mathrm{~Hz}, 1 \mathrm{H}), 4.22(\mathrm{qd}, J=6.6,1.8 \mathrm{~Hz}, 2 \mathrm{H}), 4.18-4.12$ $(\mathrm{m}, 4 \mathrm{H}), 1.25(\mathrm{t}, J=7.2 \mathrm{~Hz}, 3 \mathrm{H}), 1.22(\mathrm{t}, J=7.2 \mathrm{~Hz}, 3 \mathrm{H}), 1.19(\mathrm{t}, J=6.6 \mathrm{~Hz}, 3 \mathrm{H}) ;{ }^{13} \mathrm{C}$ NMR $\left(150 \mathrm{MHz}, \mathrm{CDCl}_{3}\right) \delta 169.6,168.9,161.1,139.5,66.1,62.3,61.9,61.2,52.2,13.9,13.8,13.7 \mathrm{IR}$ (ATR) 3293, 2982, 1724, 1697, 1545, 1337, 1213, 1127, 632, $569 \mathrm{~cm}^{-1}$; HRMS ESI $m / z\left[\mathrm{MH}^{+}\right]$ calcd for $\mathrm{C}_{12} \mathrm{H}_{19} \mathrm{~N}_{2} \mathrm{O}_{6}$ : 287.1238. Found: 287.1234. 


\section{${ }^{1} \mathrm{H}$ NMR and ${ }^{13} \mathrm{C}$ NMR spectra of starting compounds}
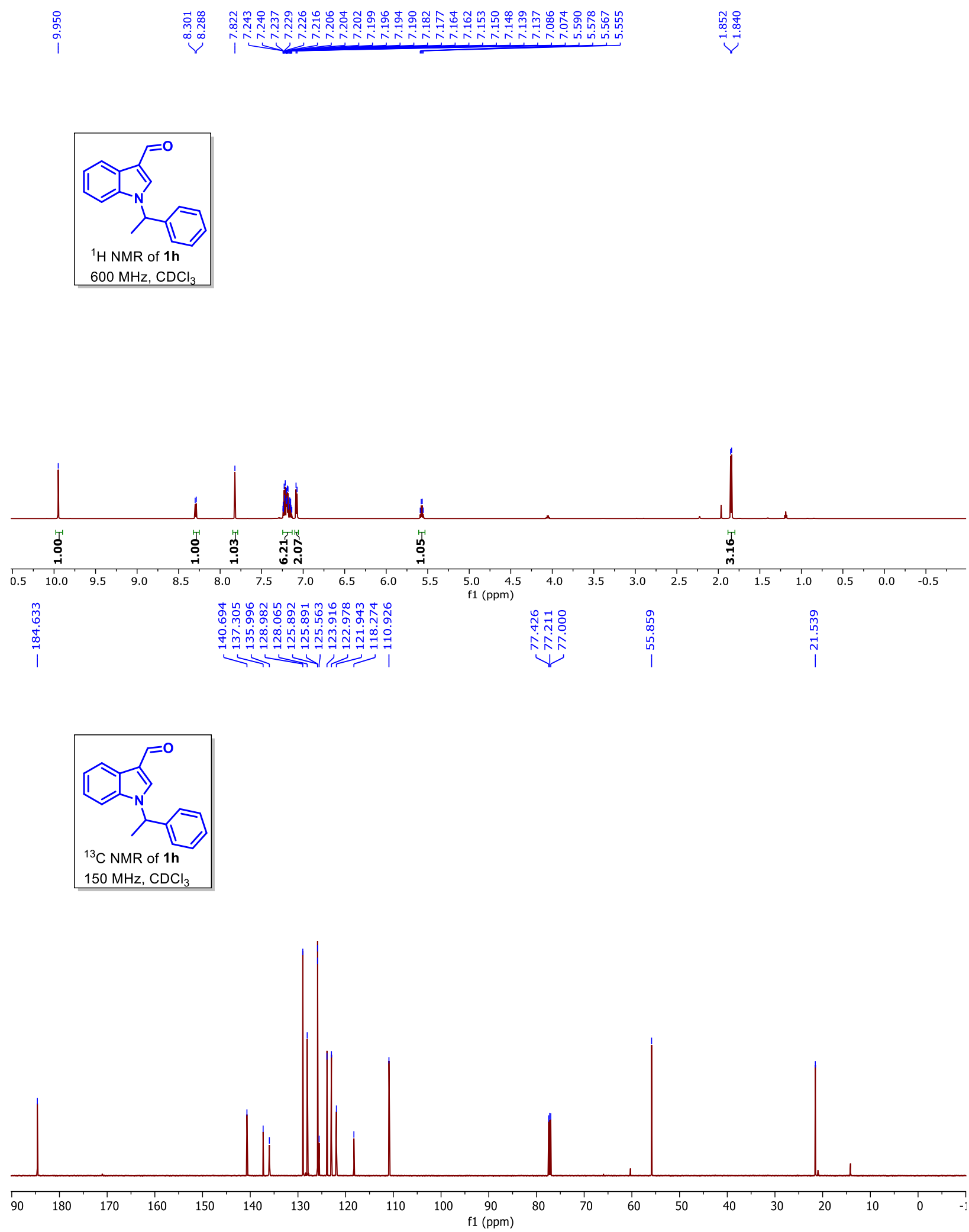
每
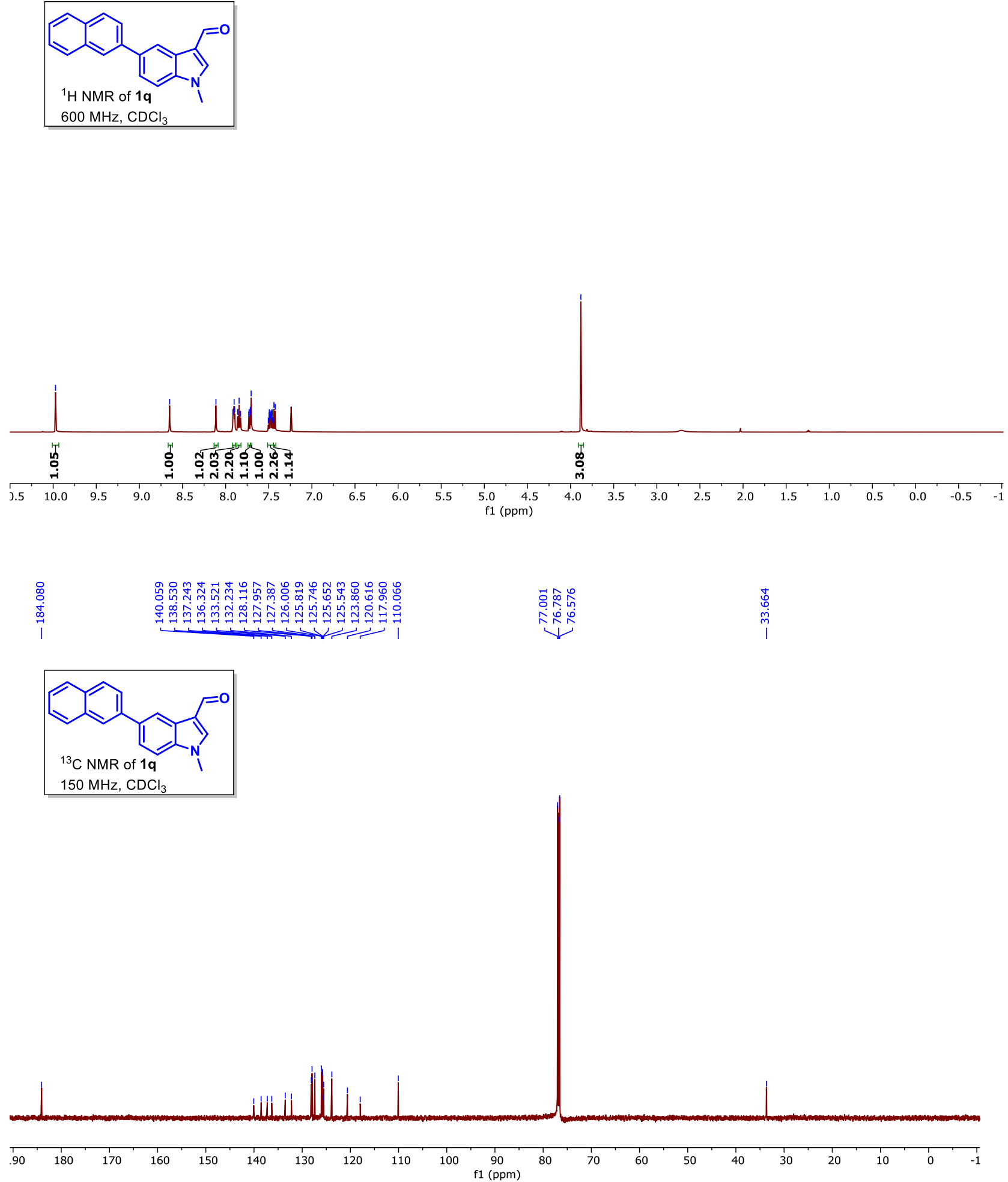

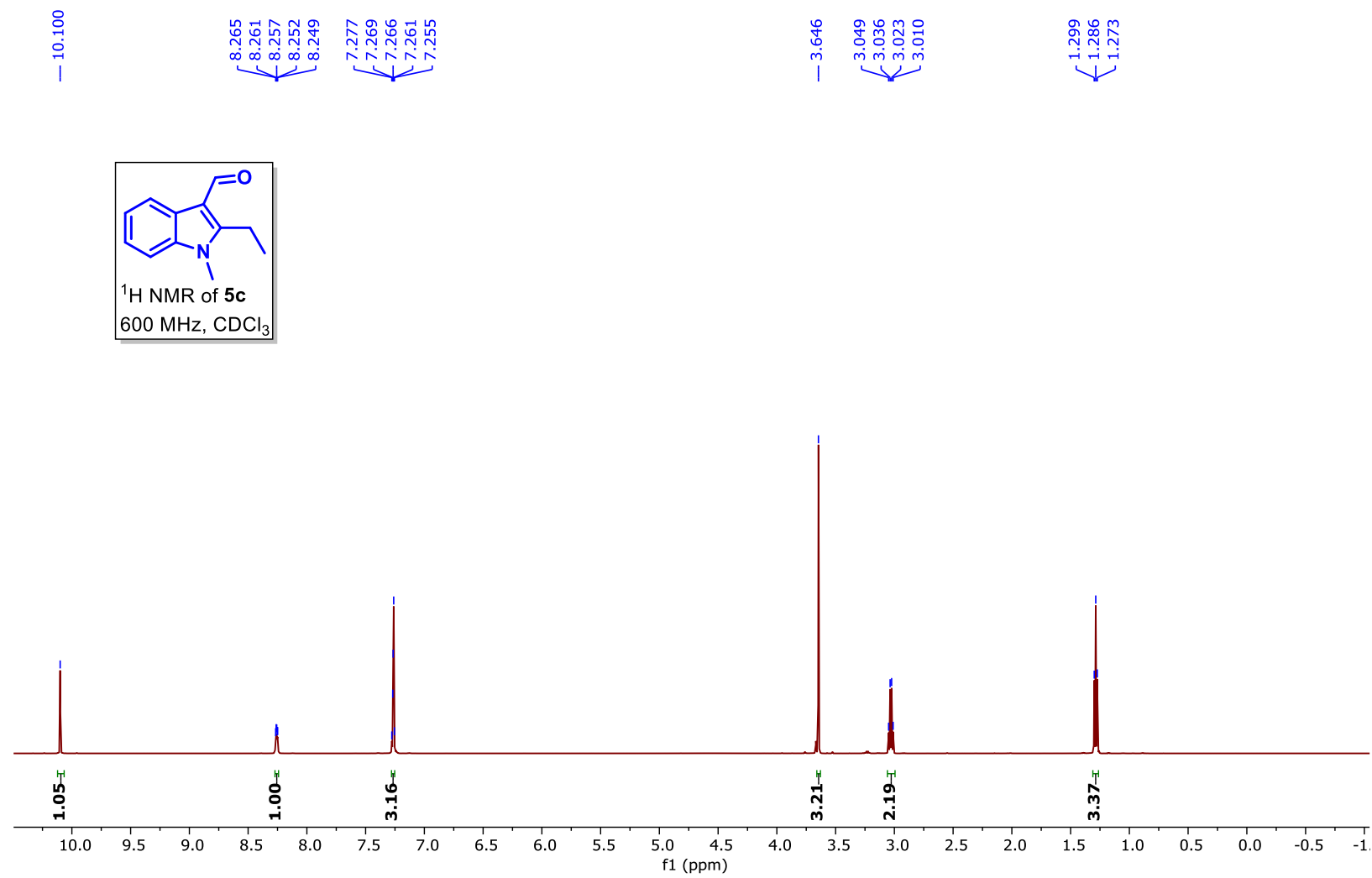

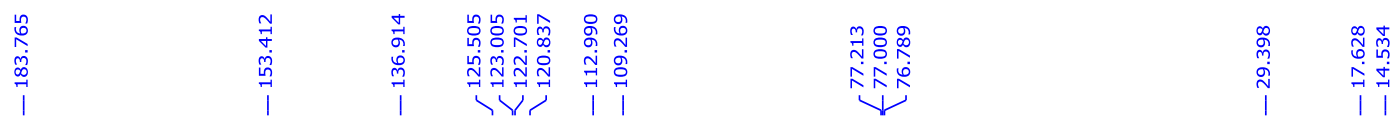
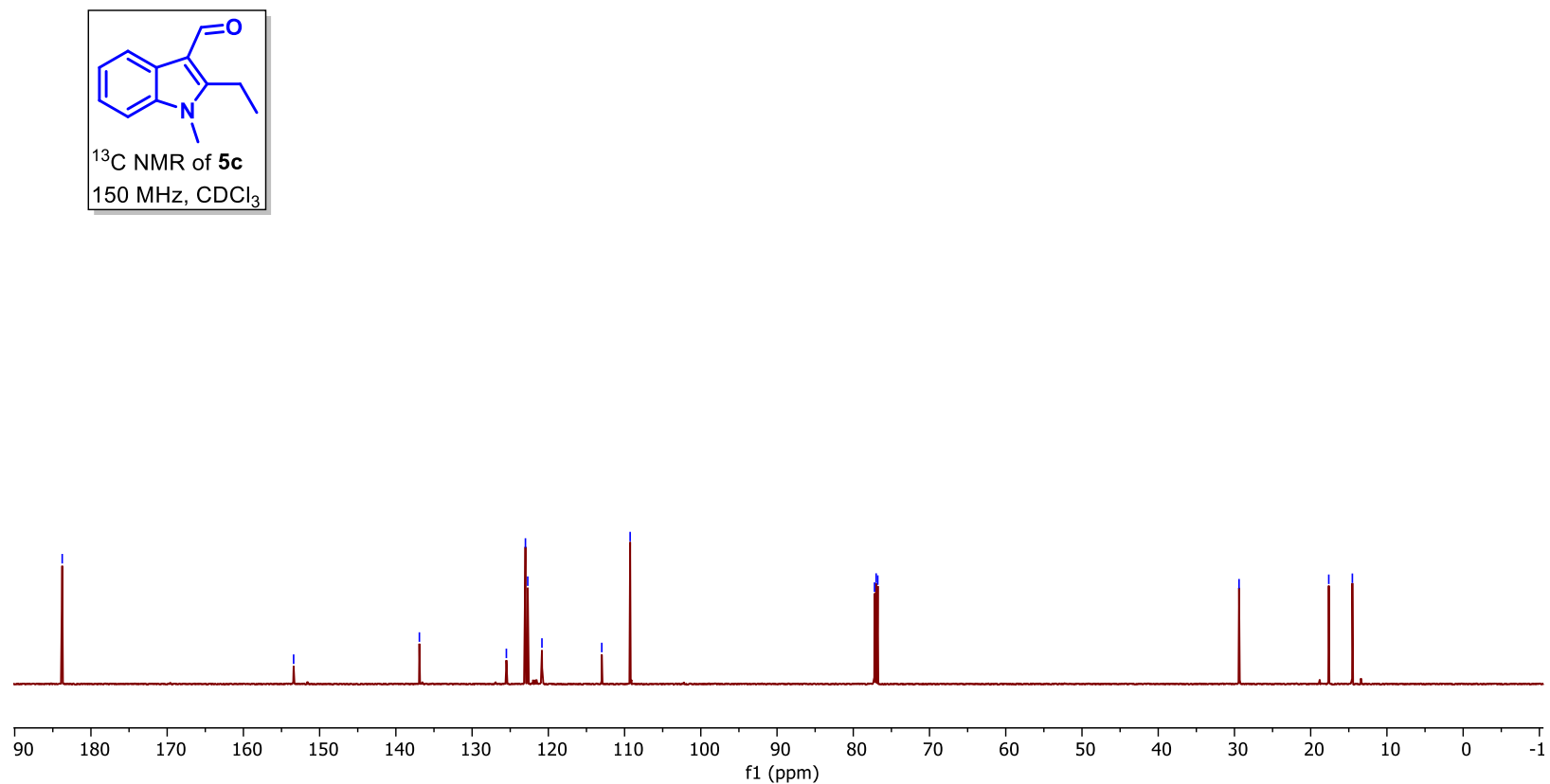


\section{${ }^{1} \mathrm{H}$ NMR and ${ }^{13} \mathrm{C}$ NMR spectra of synthesized products}
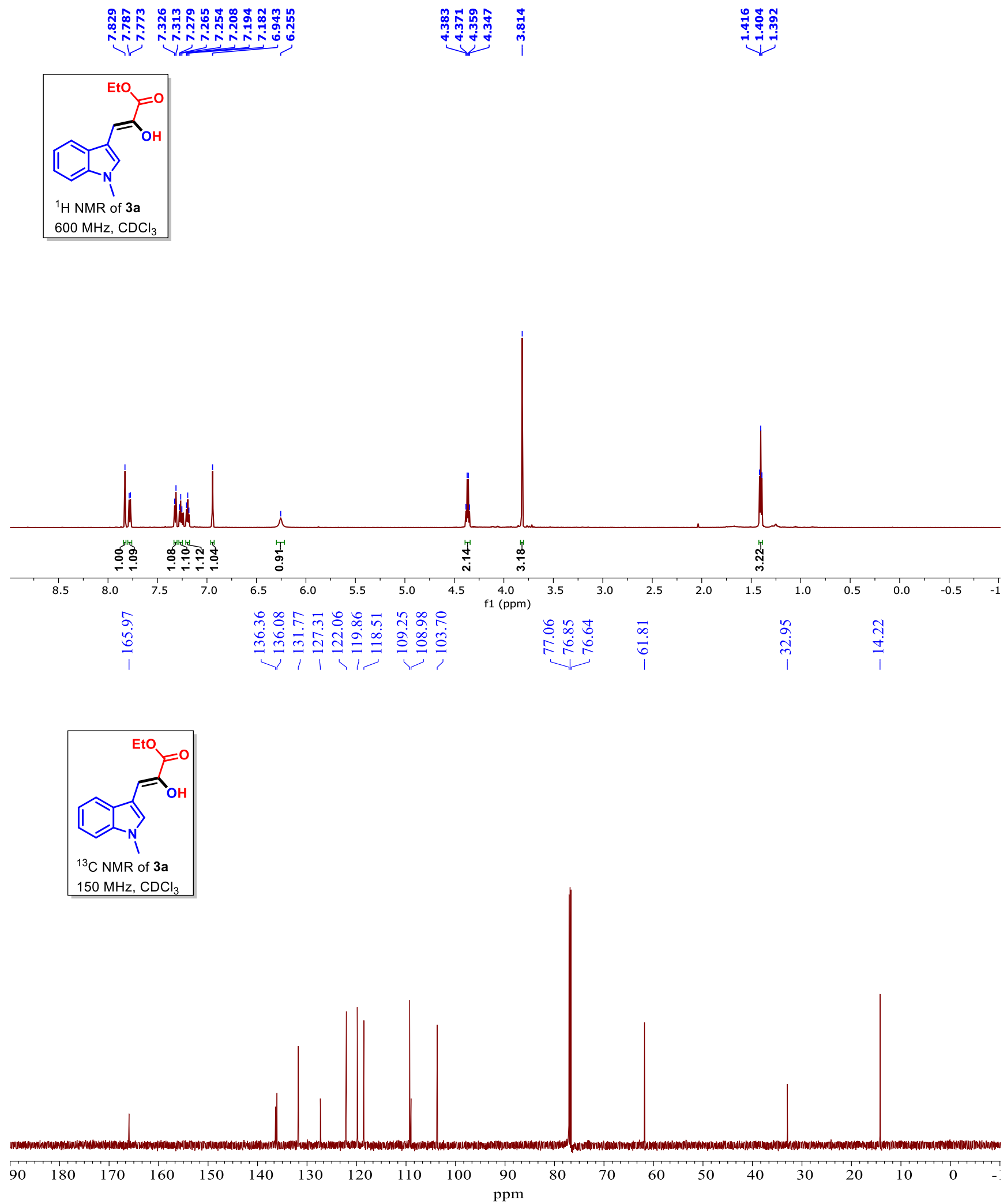

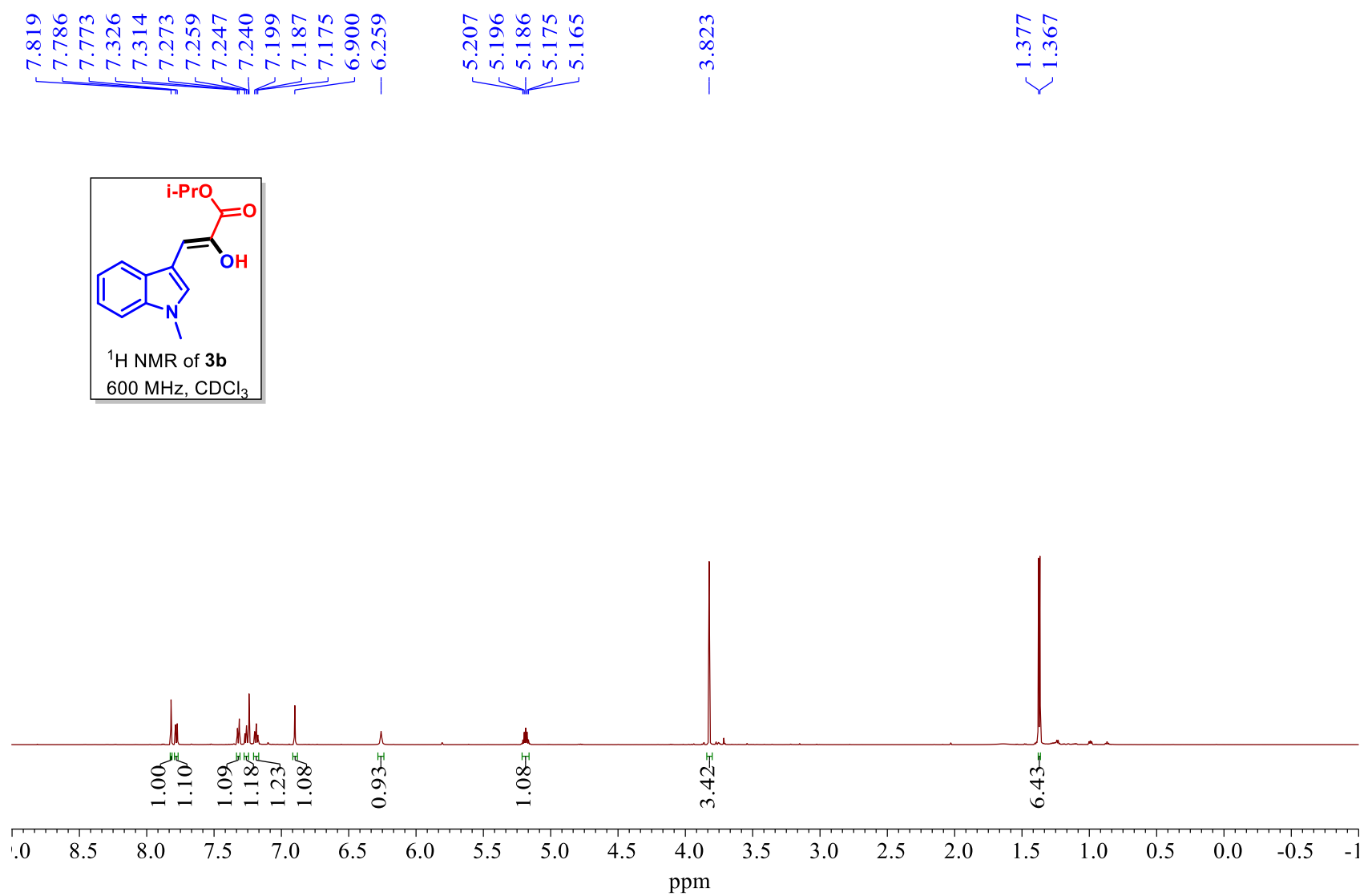

\begin{tabular}{|c|}
\hline 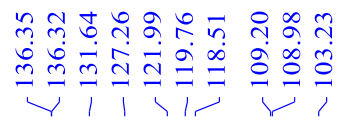 \\
\hline
\end{tabular}
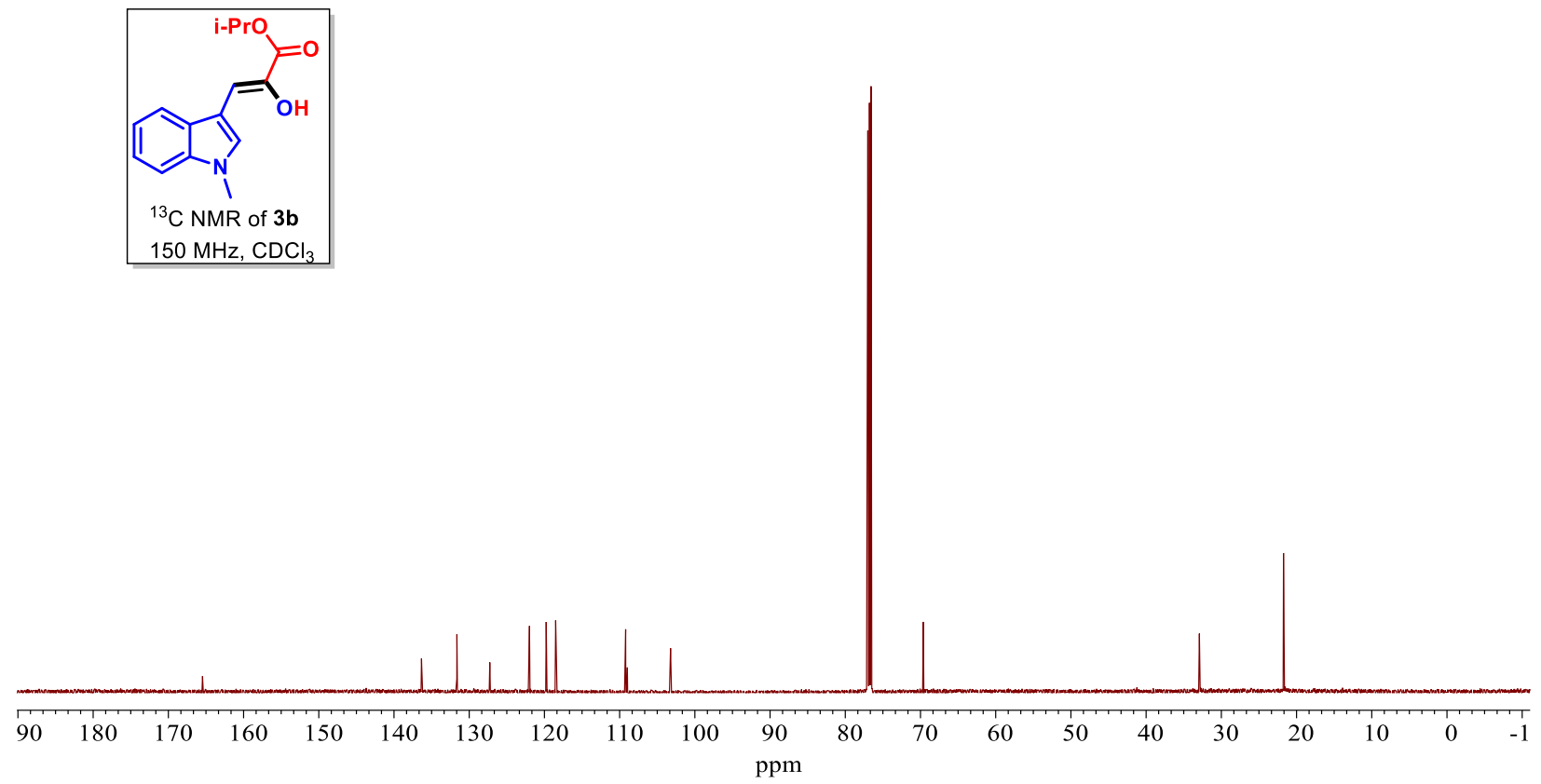

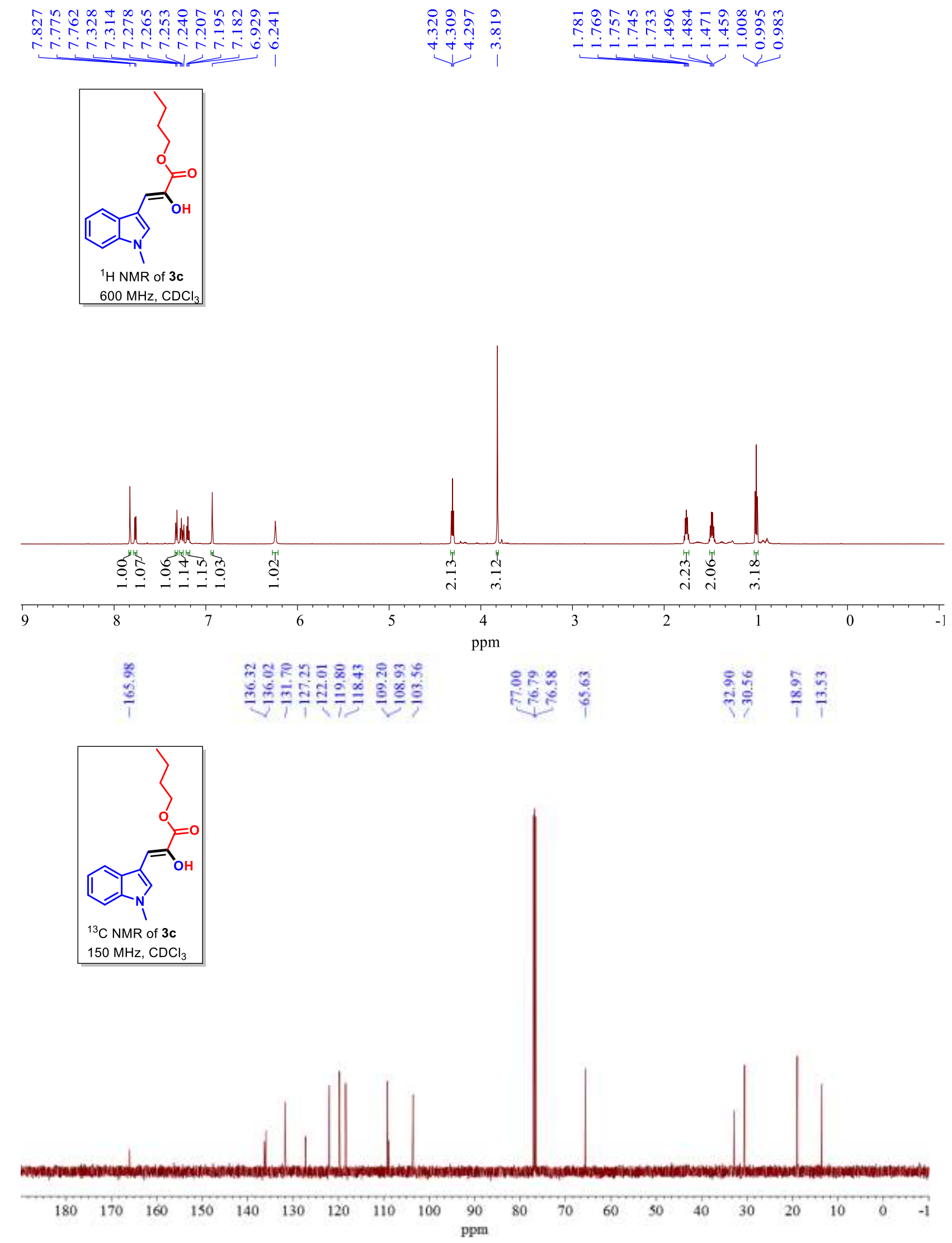


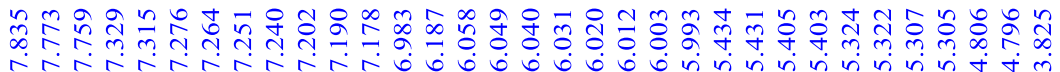
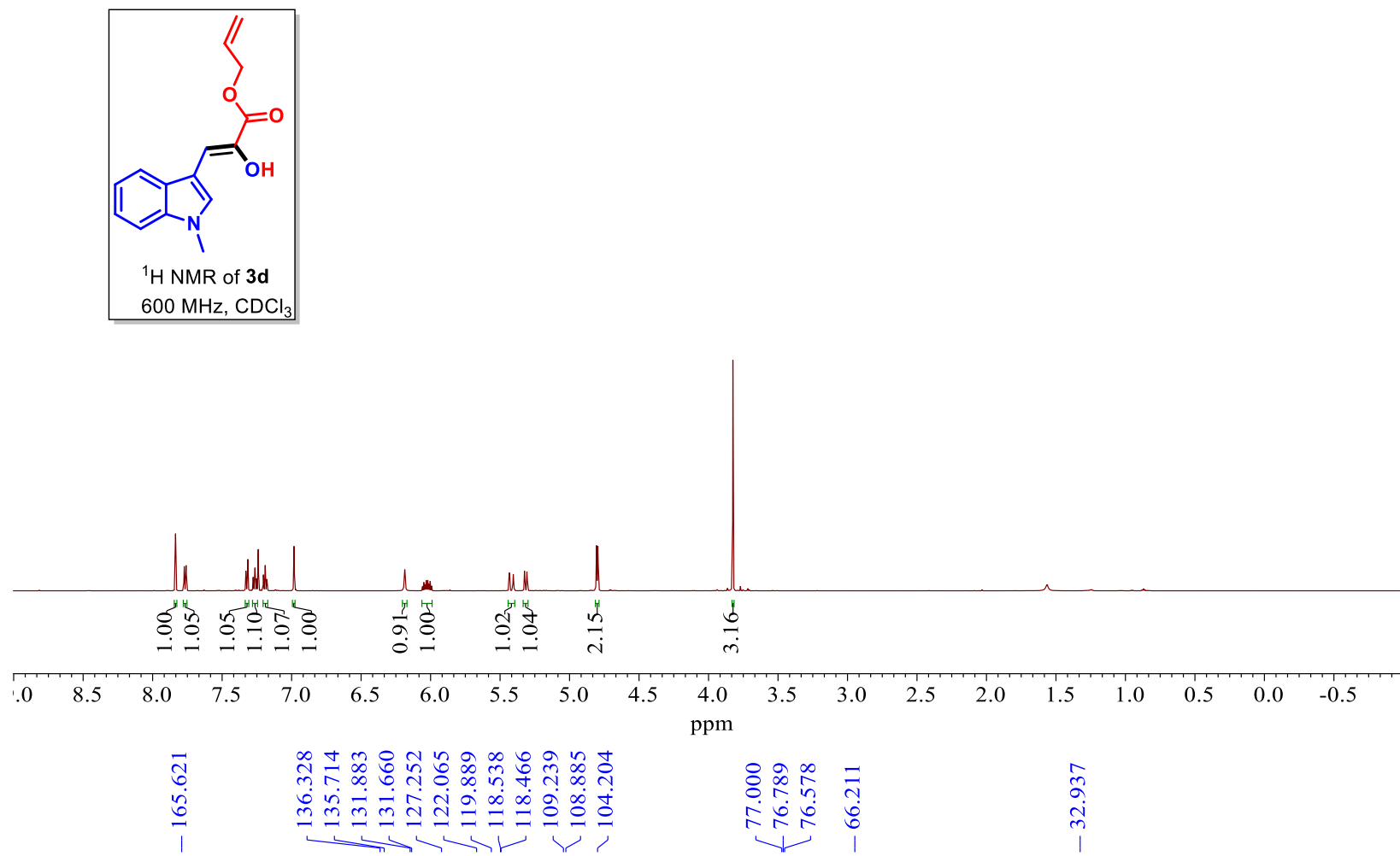

aे
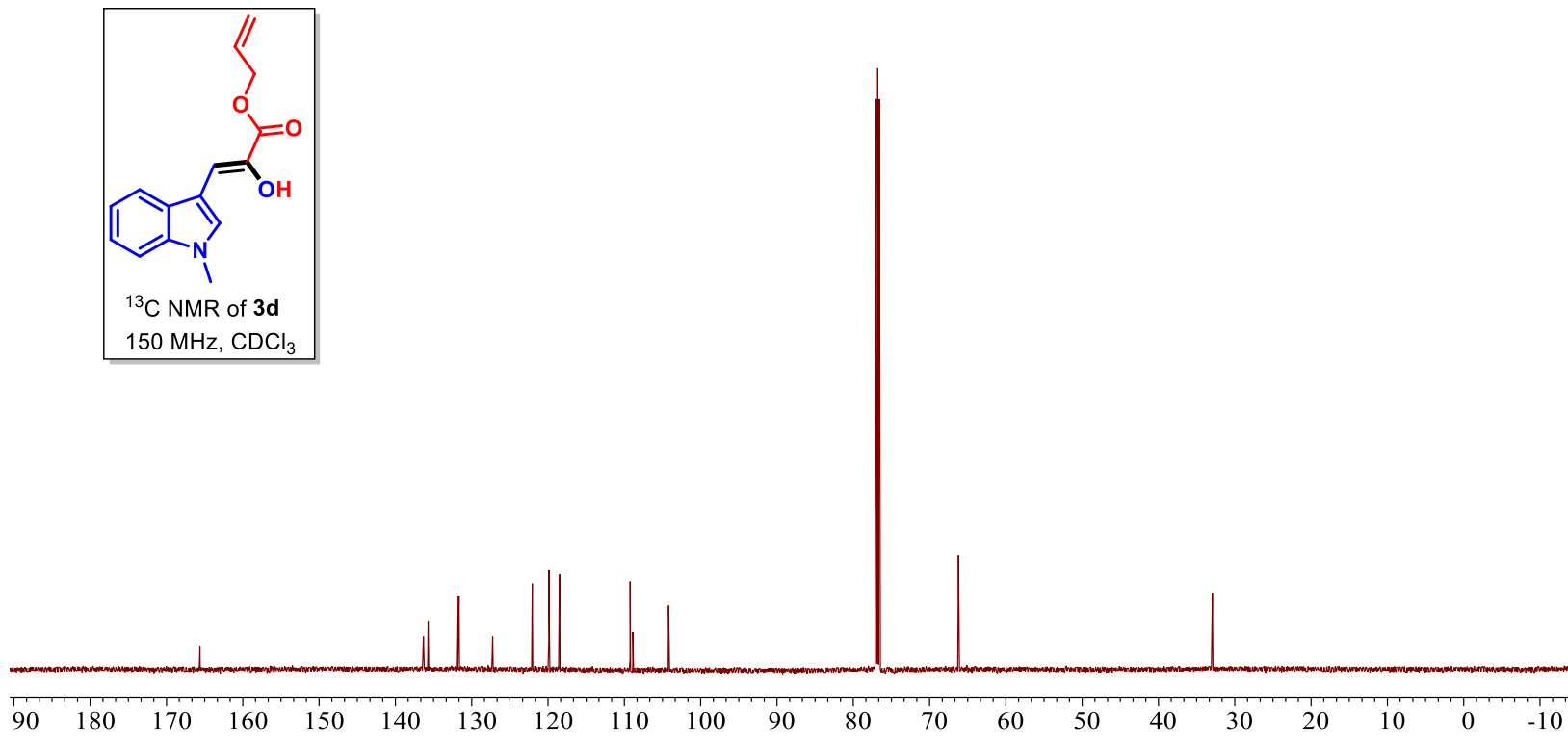


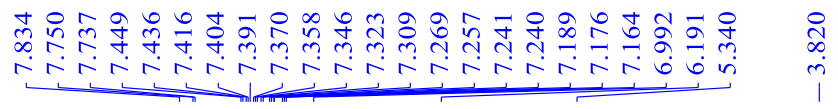
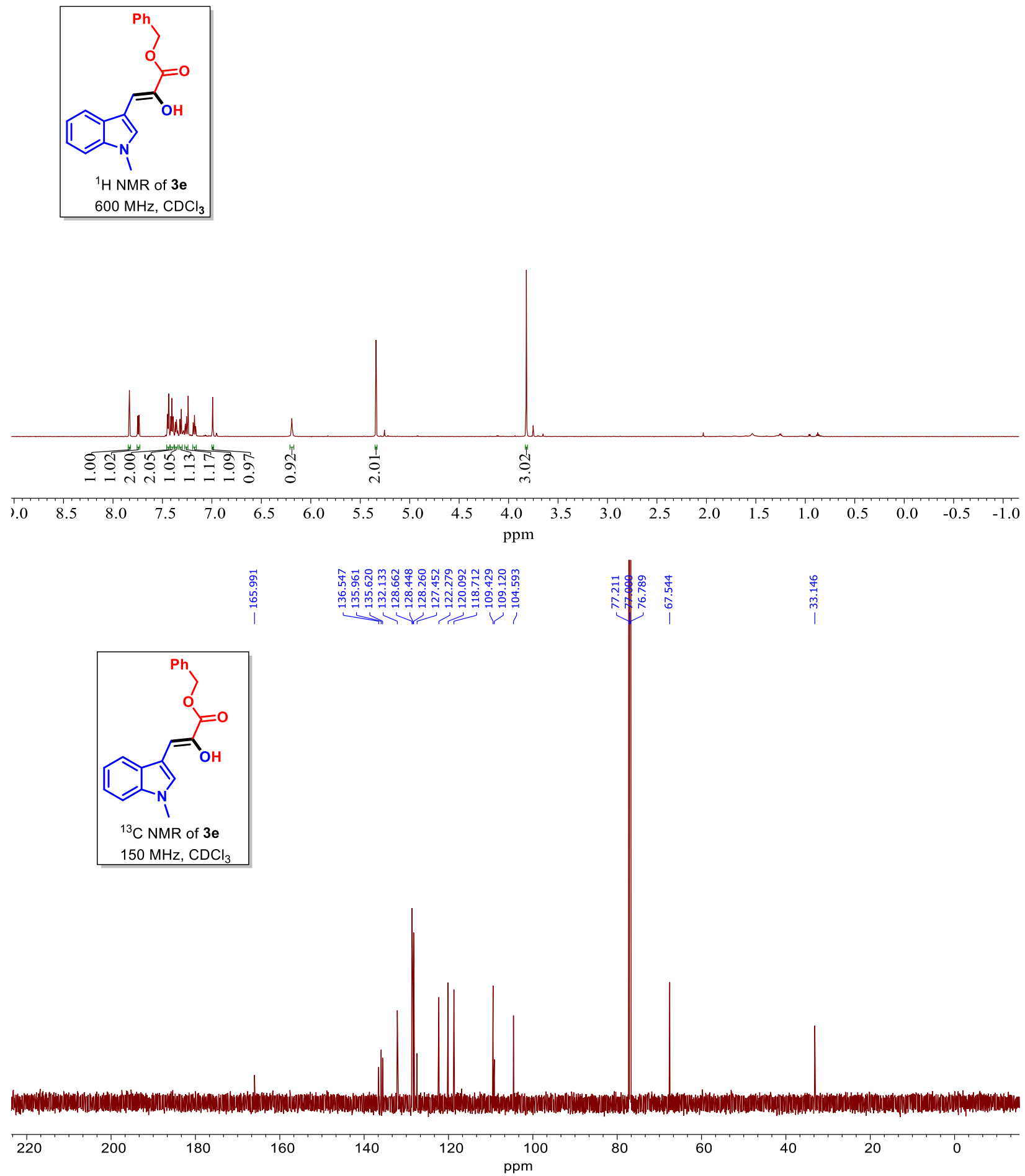

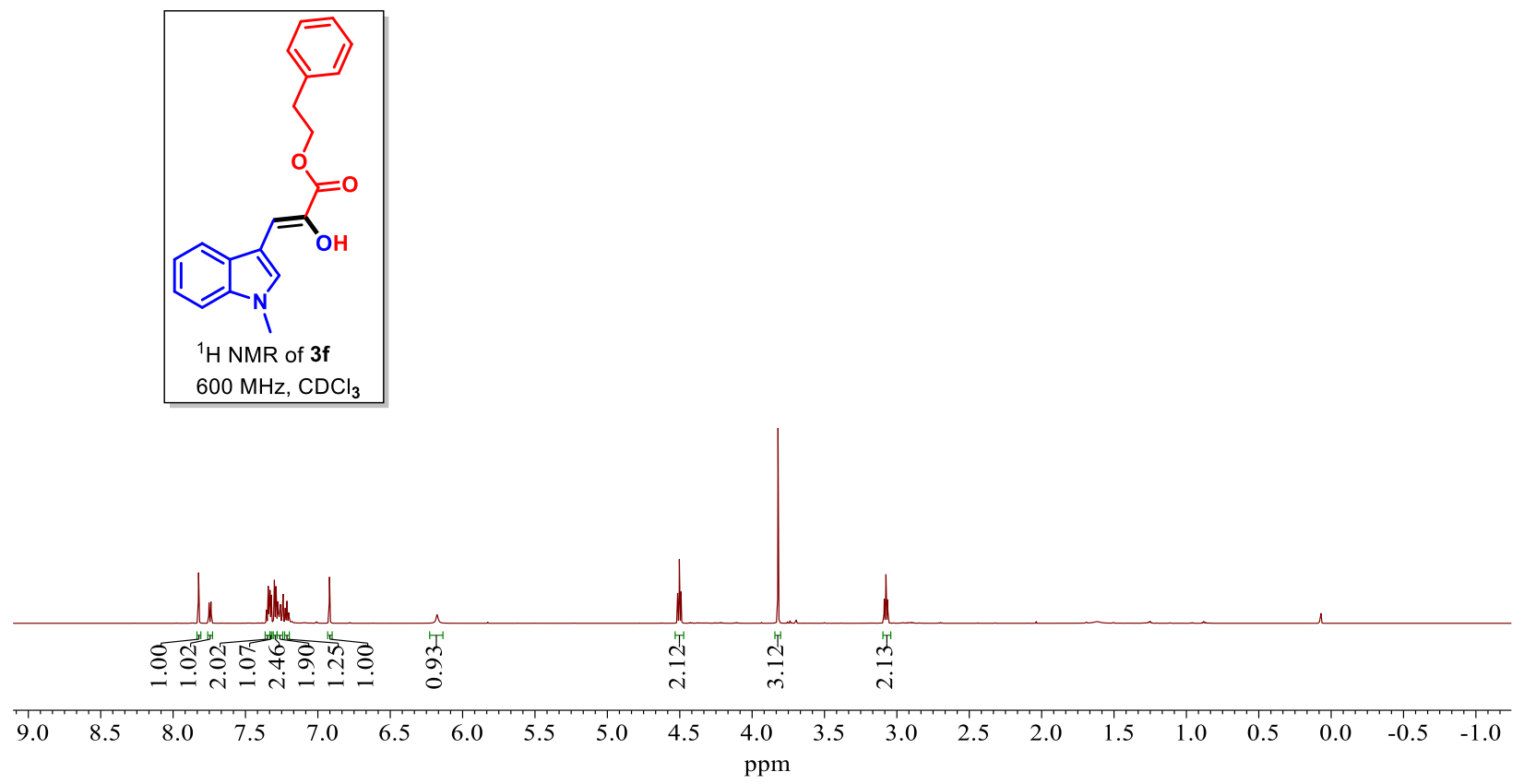

ก กิ

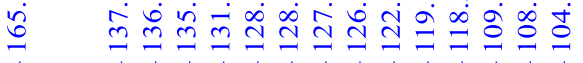

।

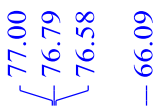

$\because \bar{a}$

mi
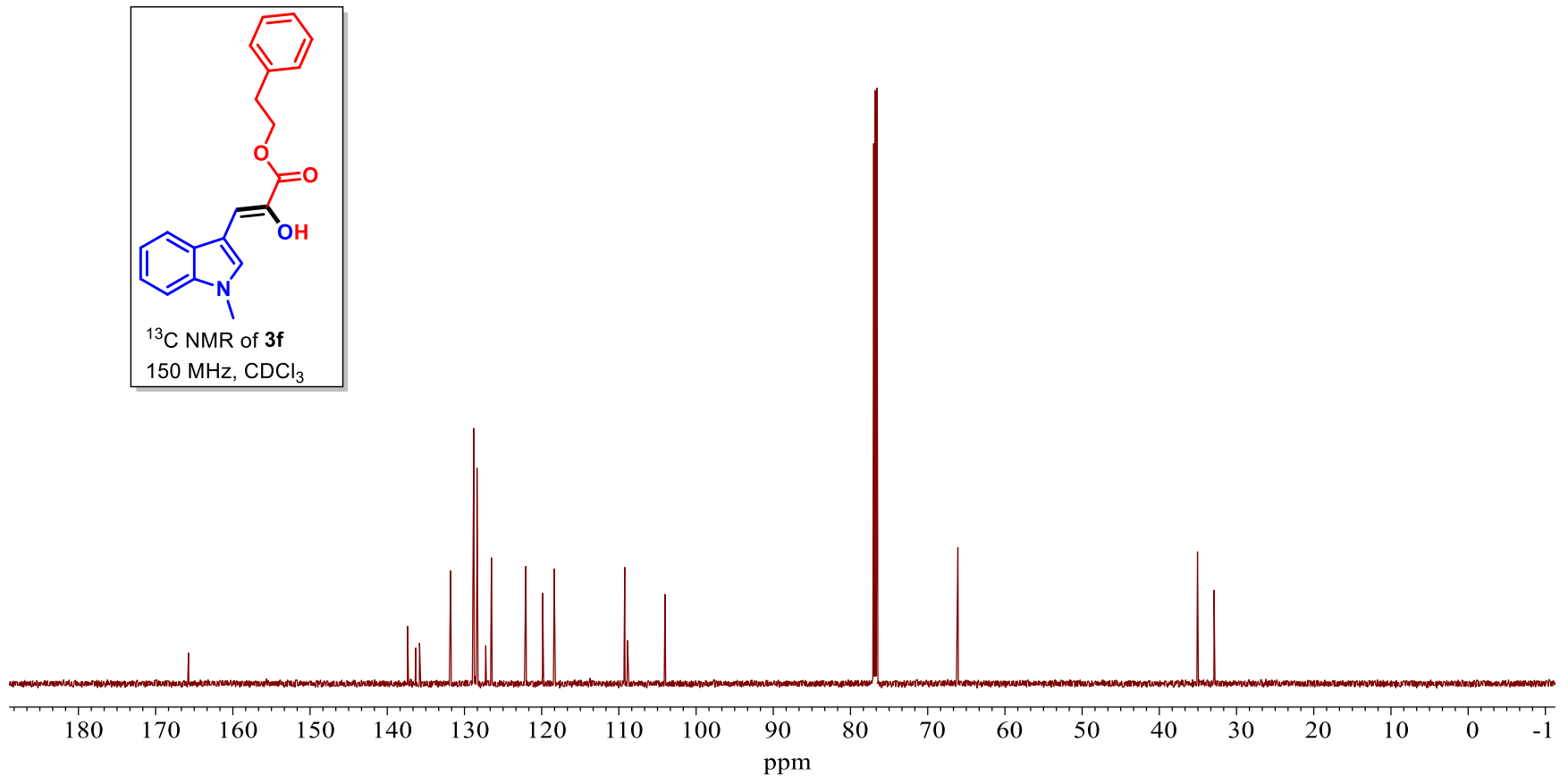


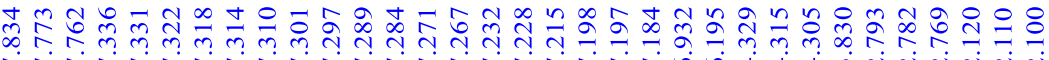

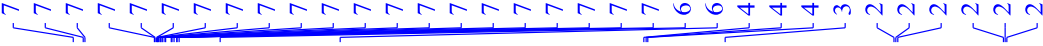

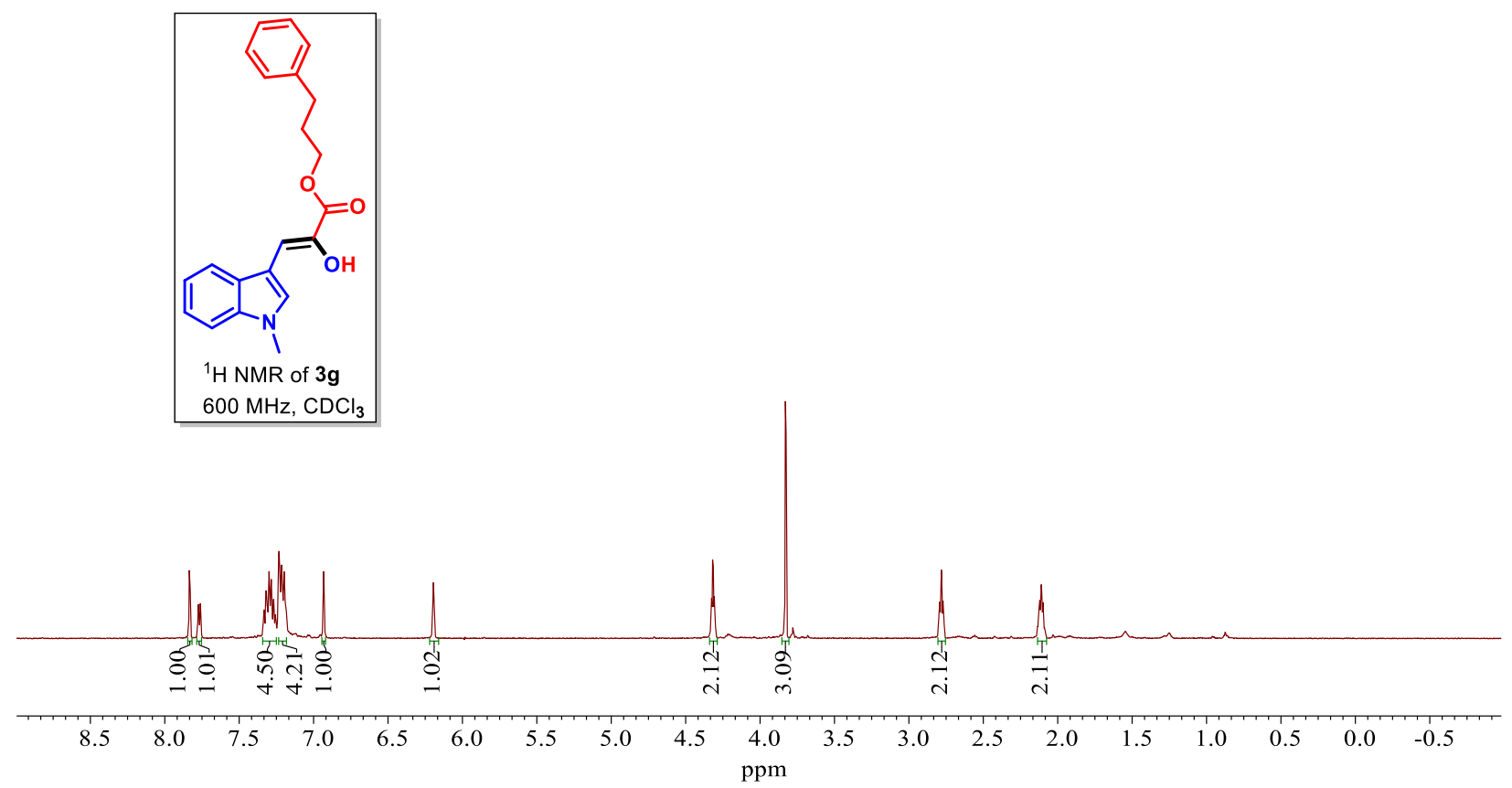

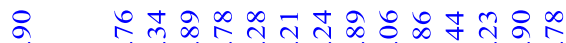

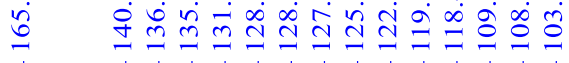

\&ุุ

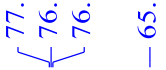

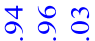

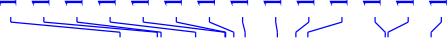

ले ले

it
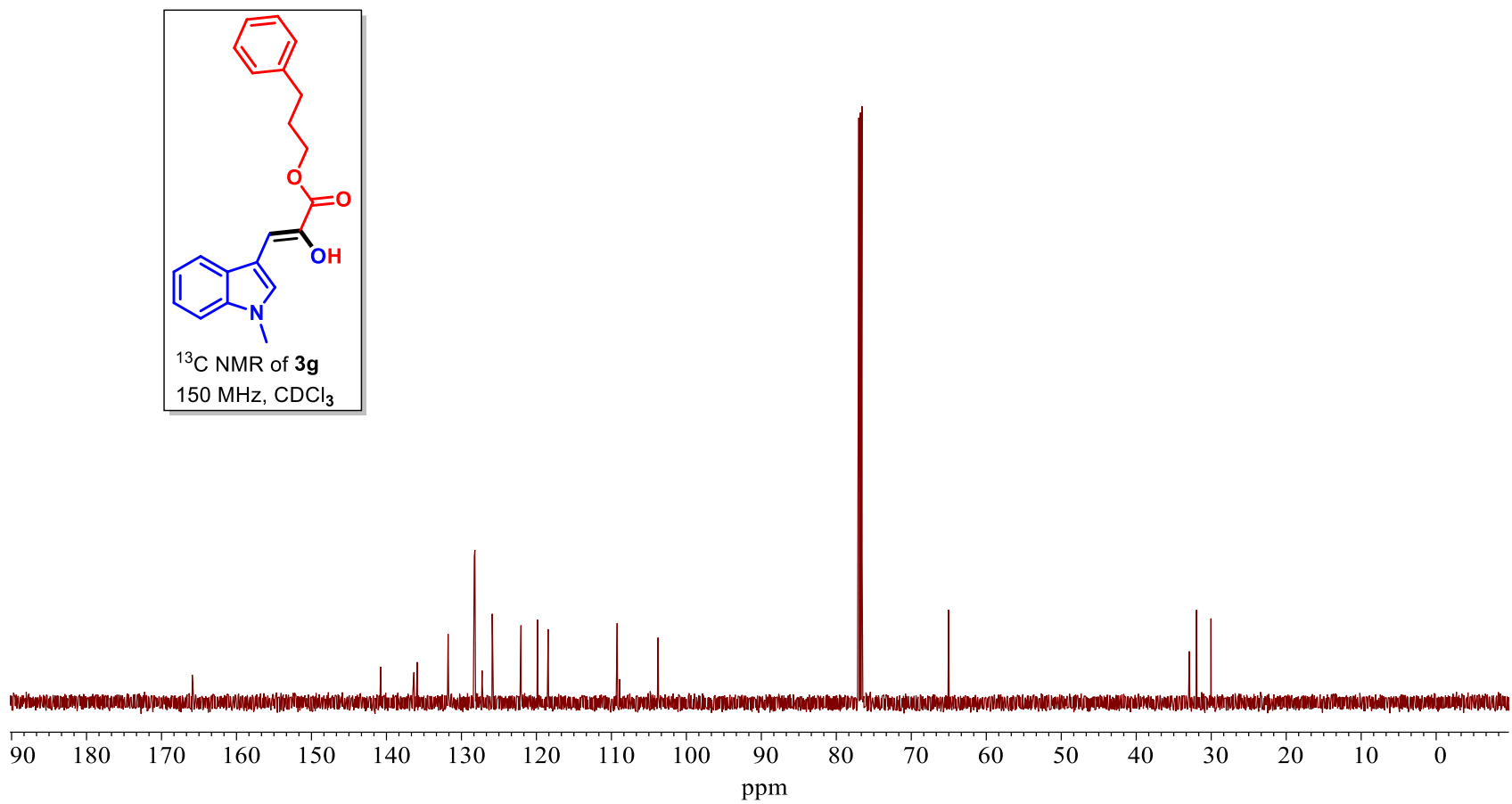

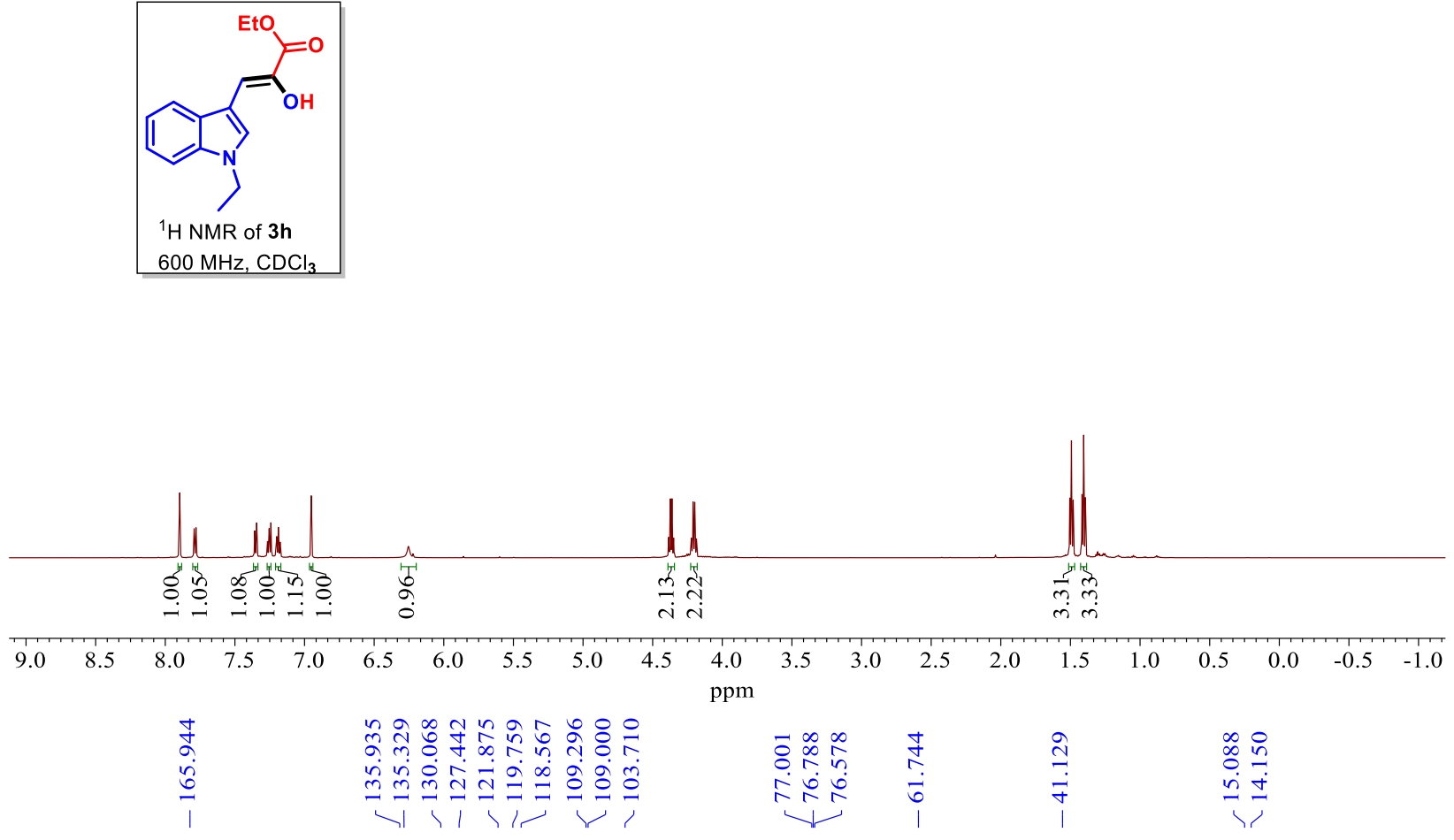

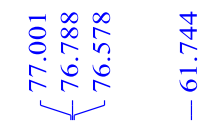

\section{$\stackrel{9}{\text { ป }}$}

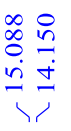

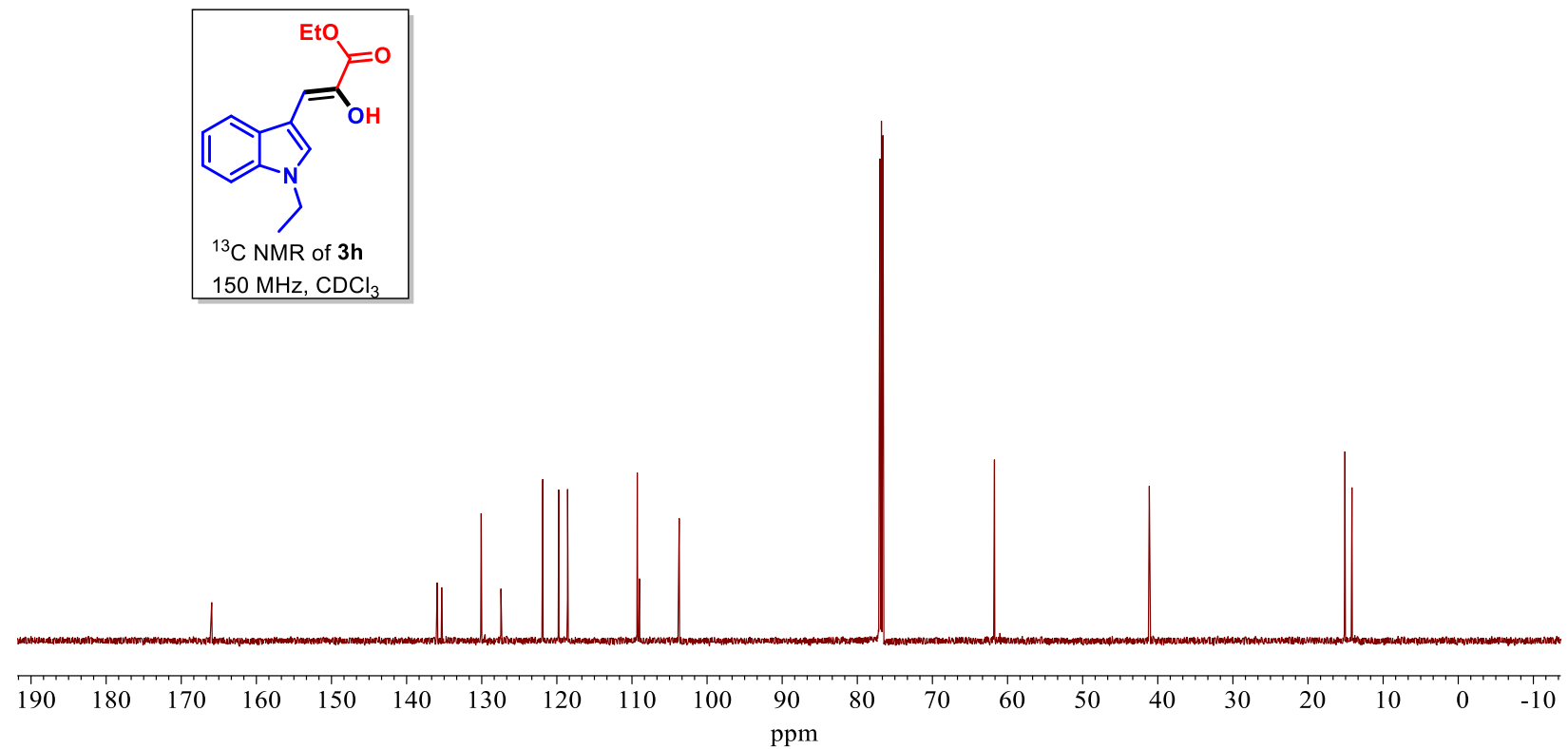




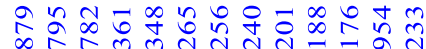

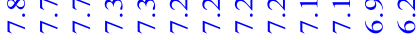

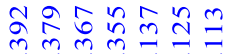

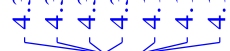

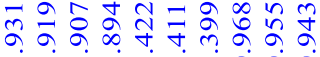

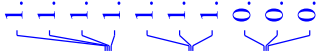
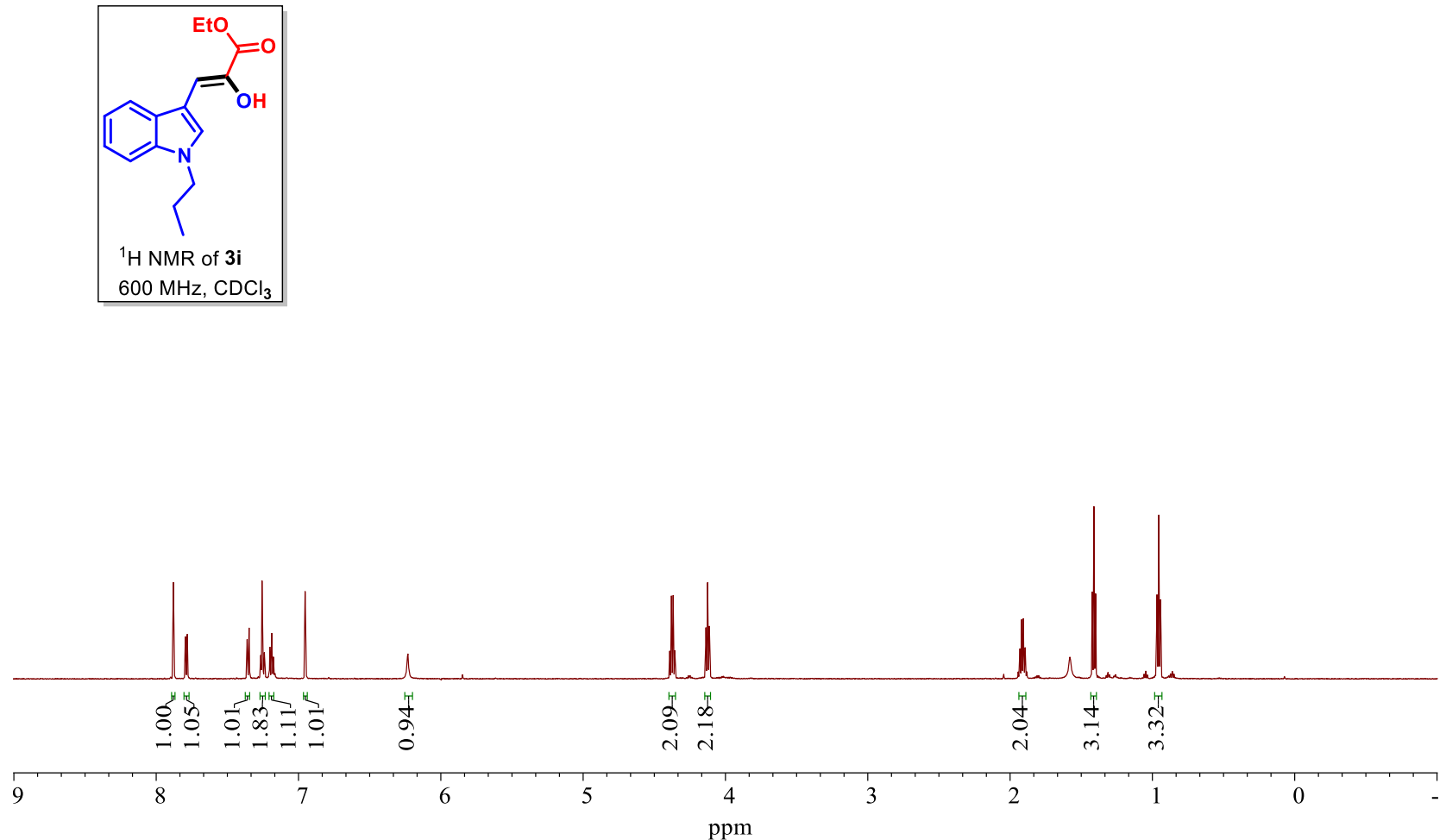

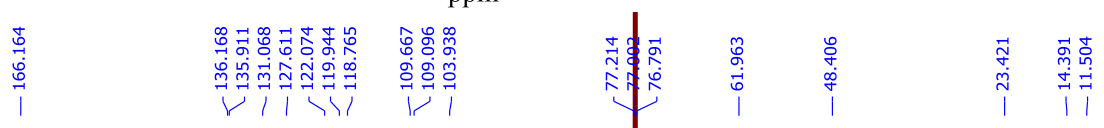
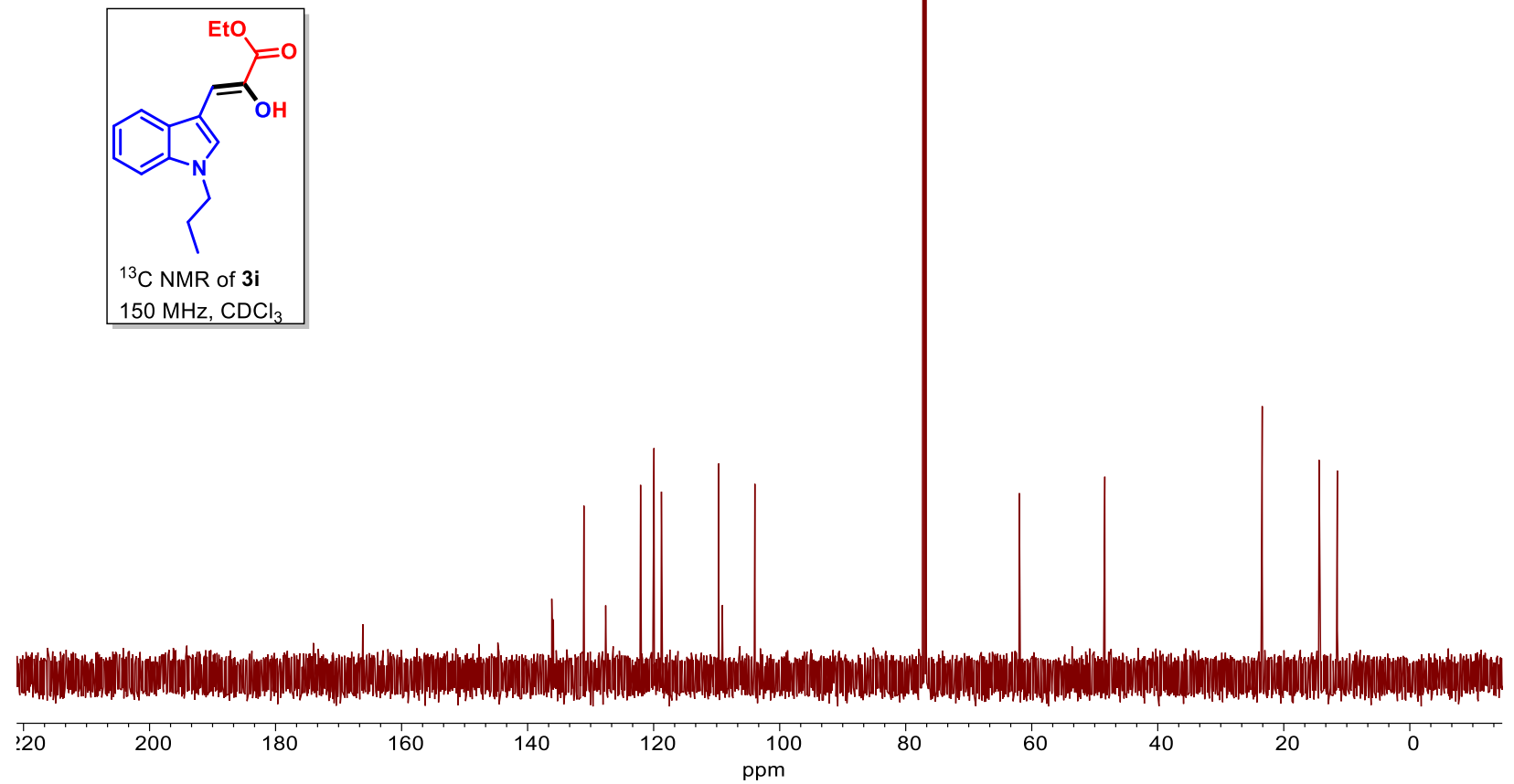

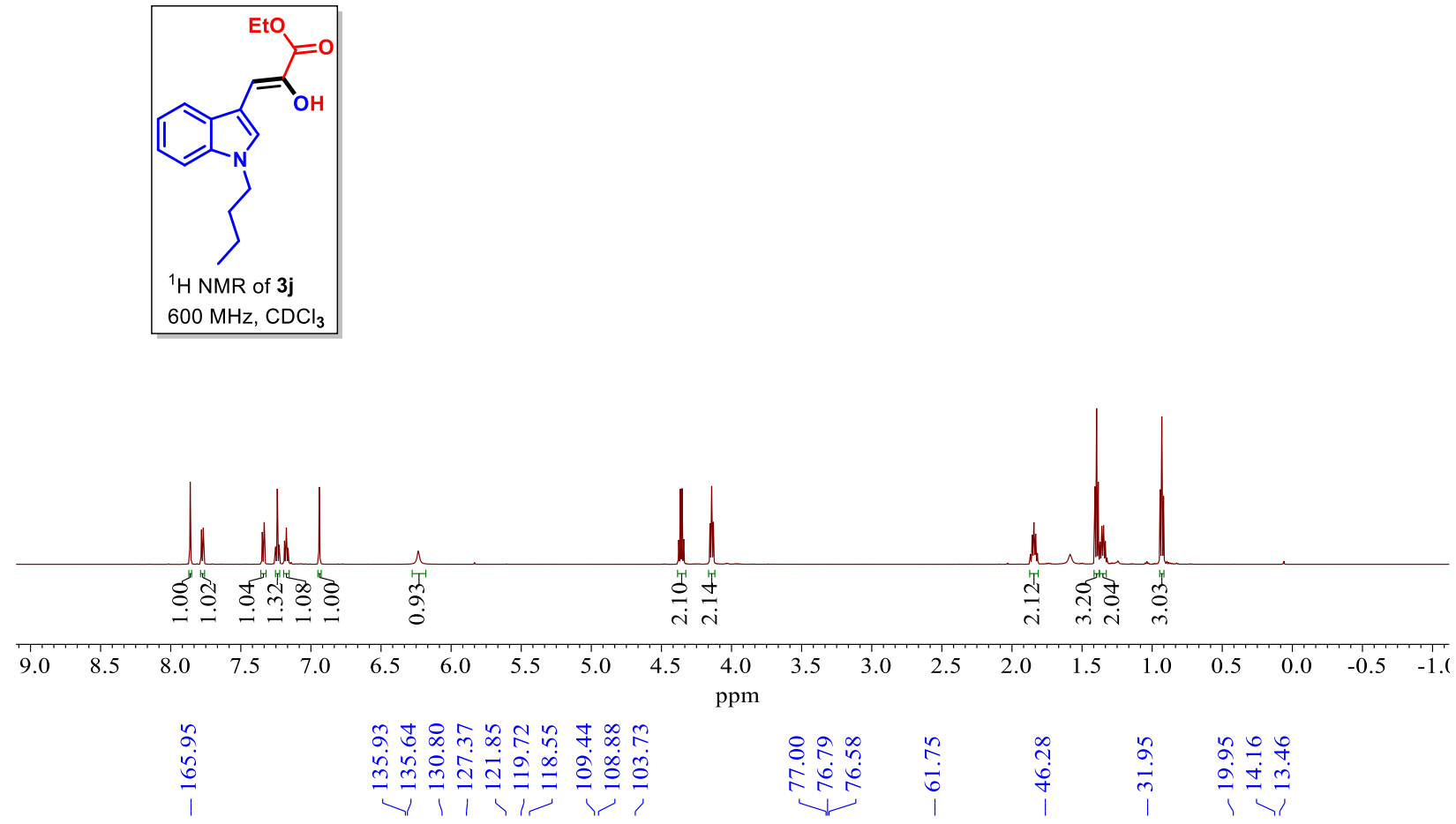

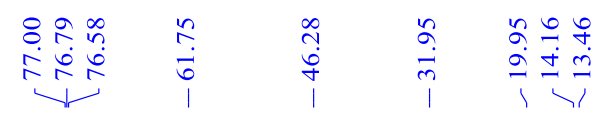

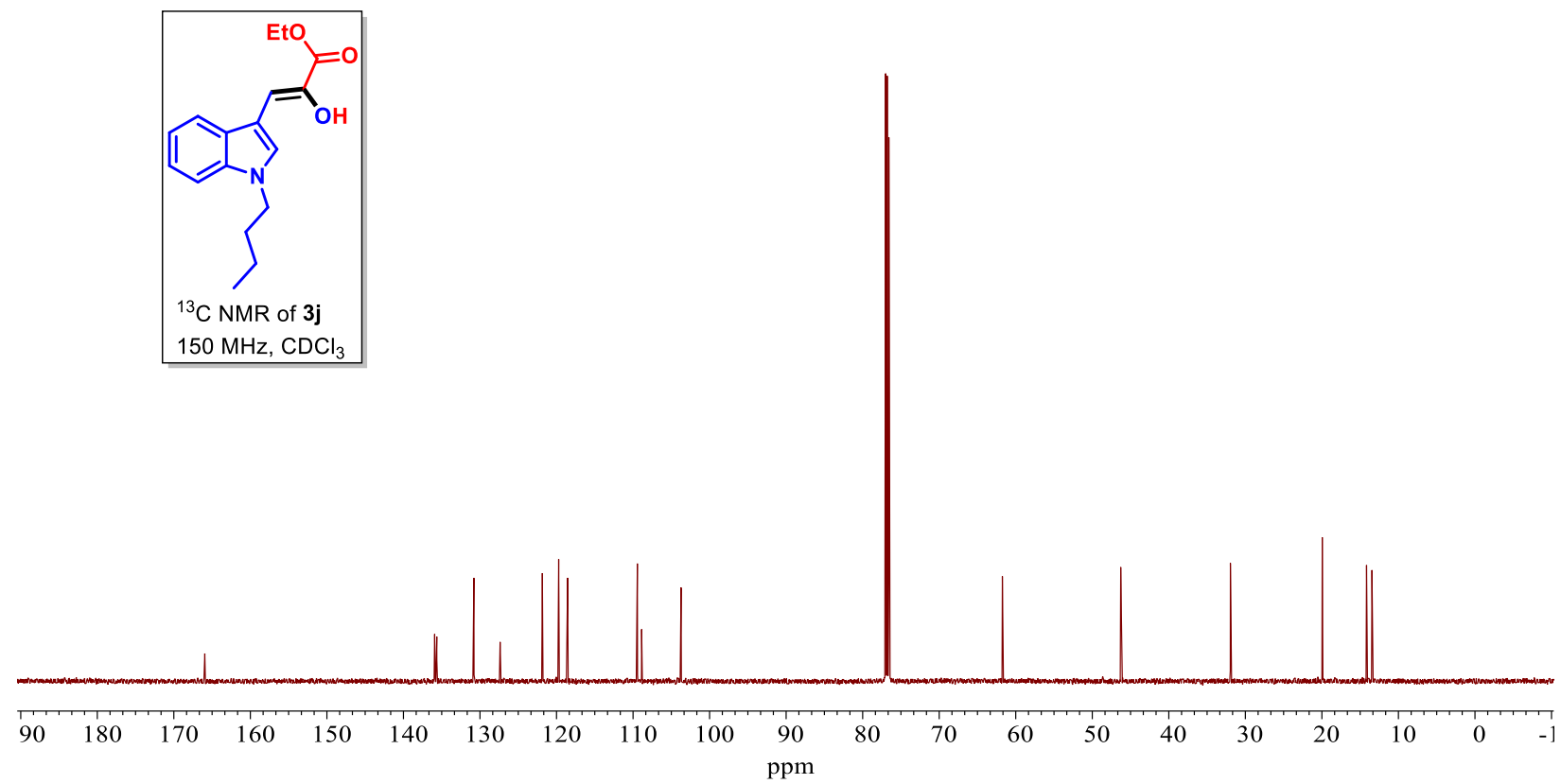




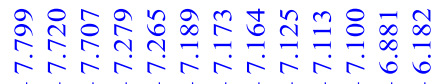

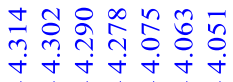
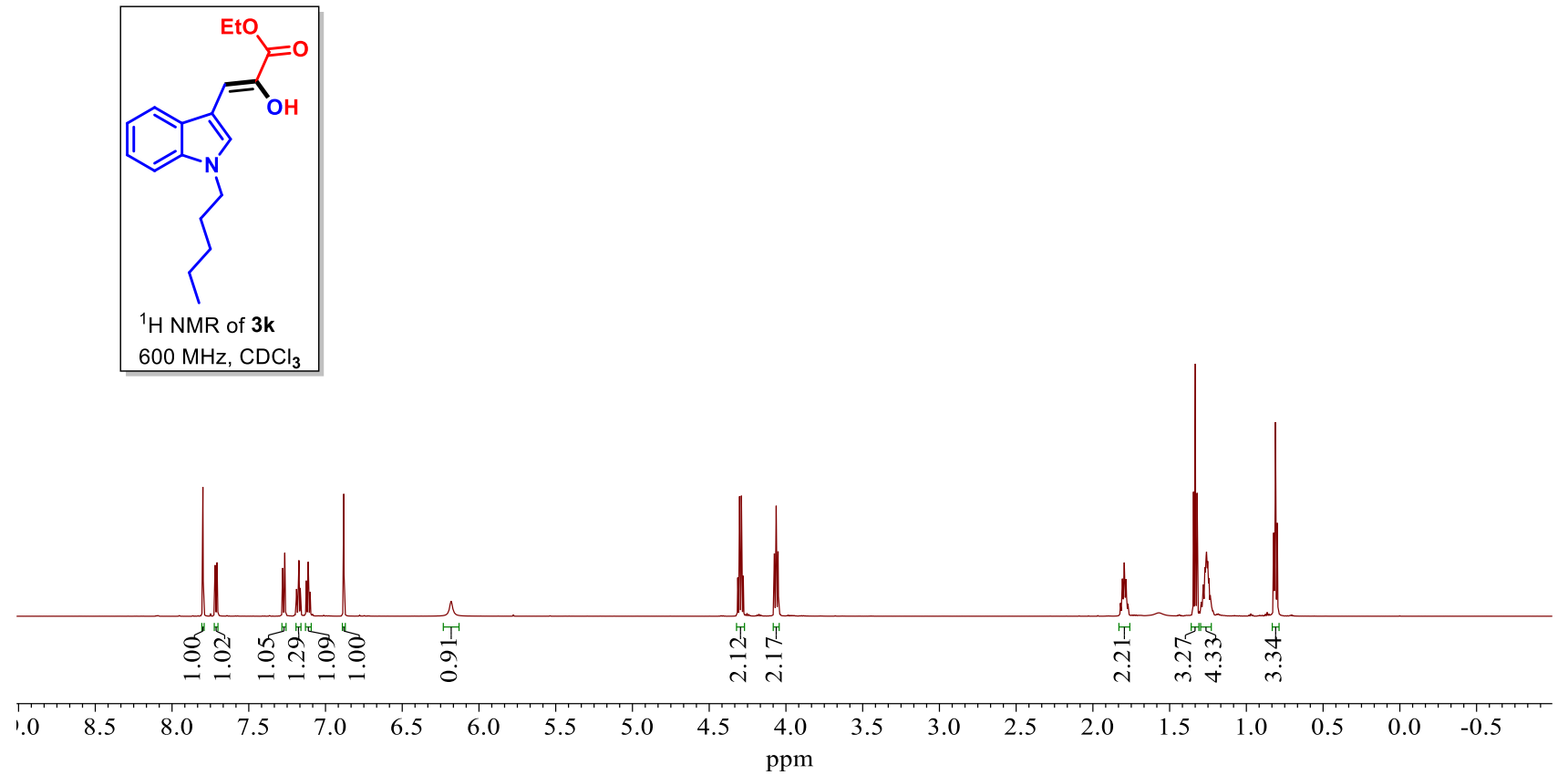

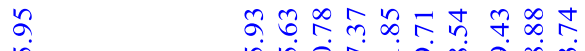

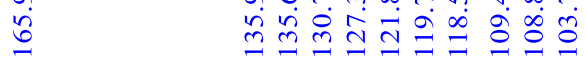

可原少,

우요

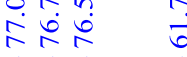

।

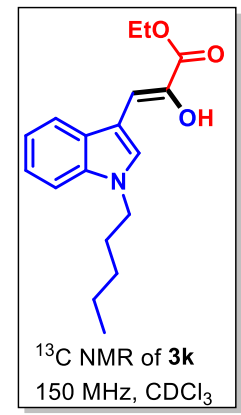

$150 \mathrm{MHz}, \mathrm{CDCl}_{3}$

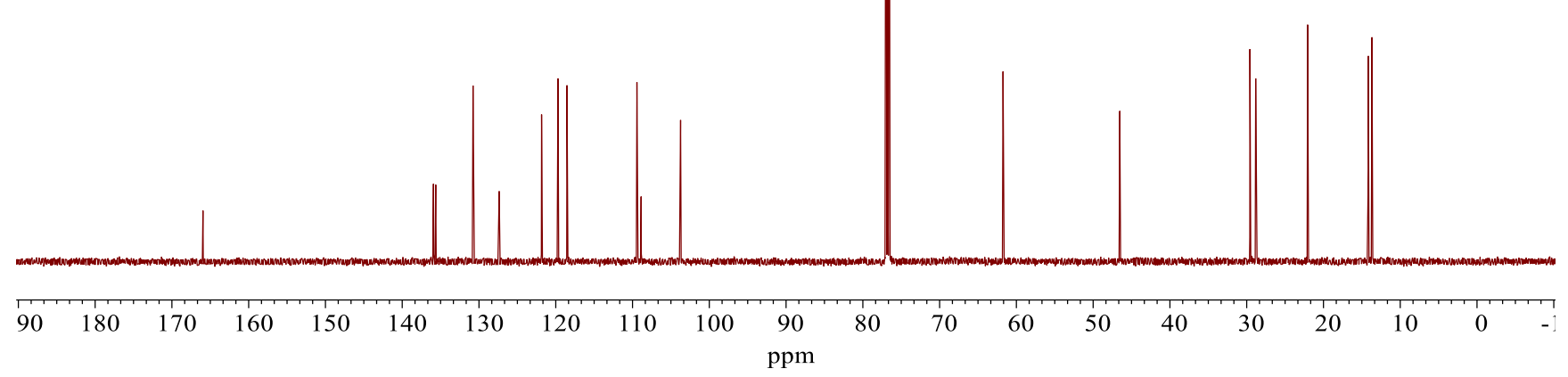



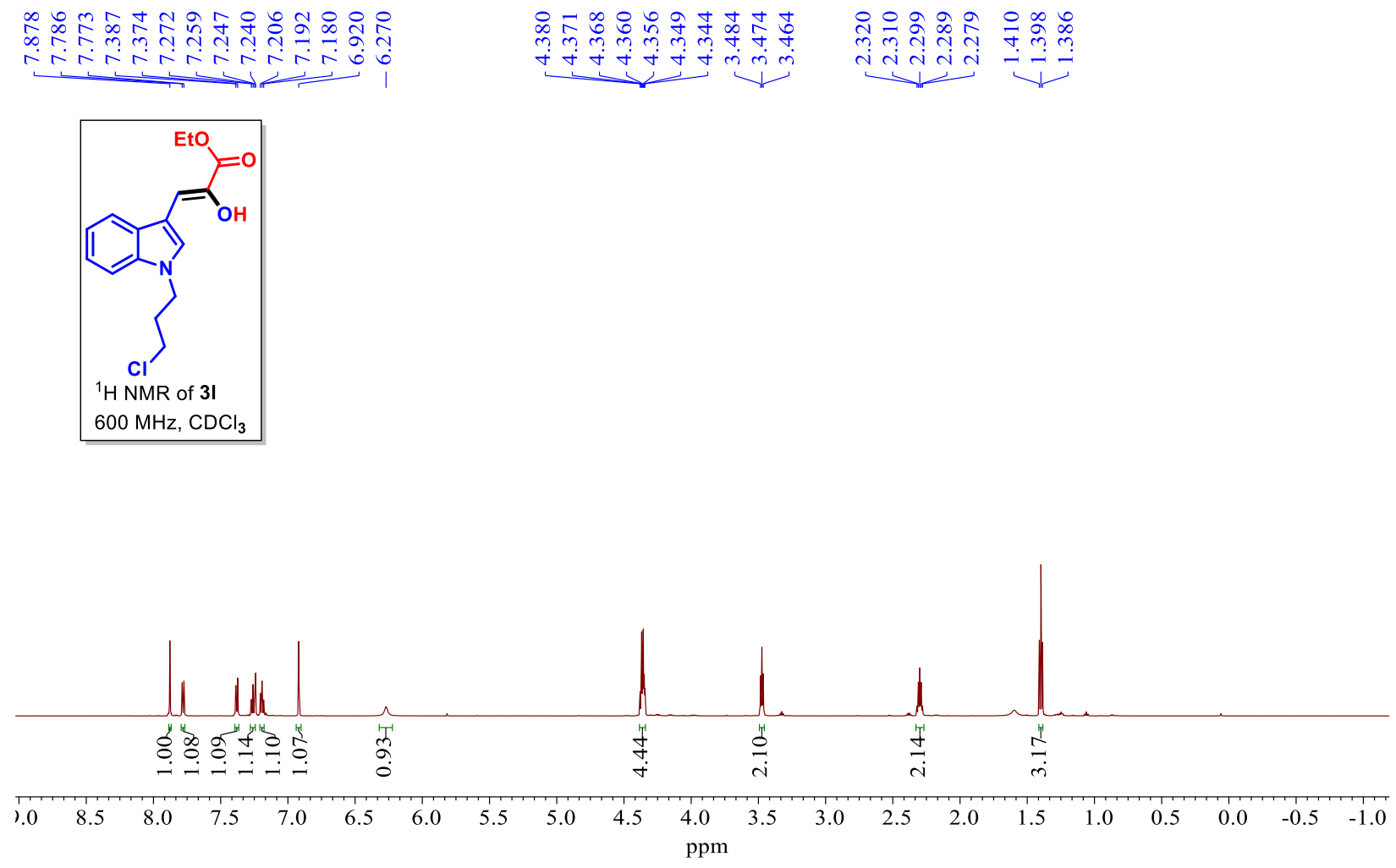

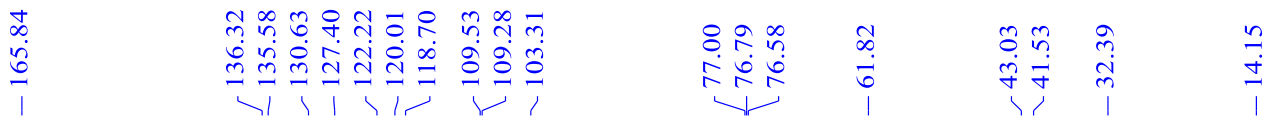
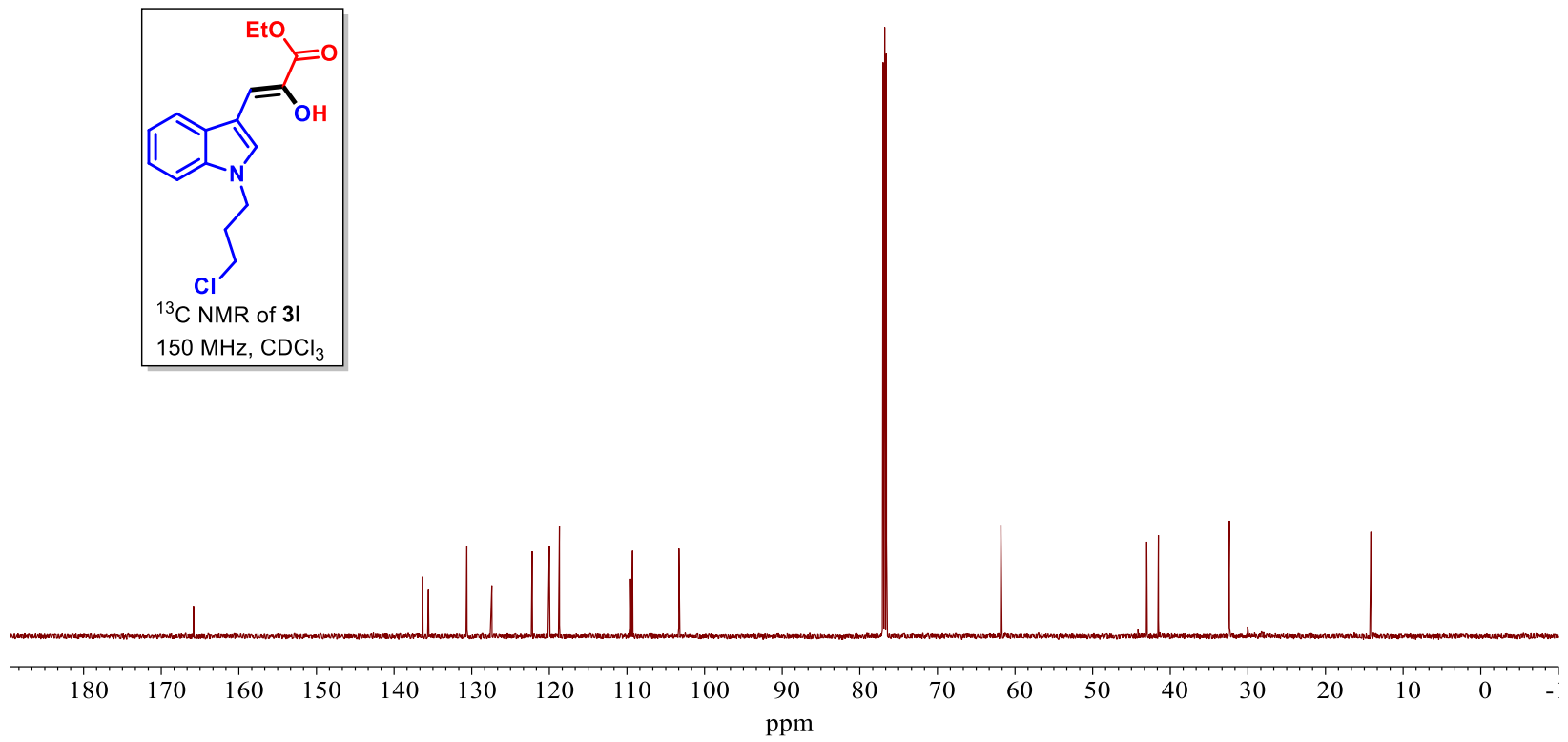


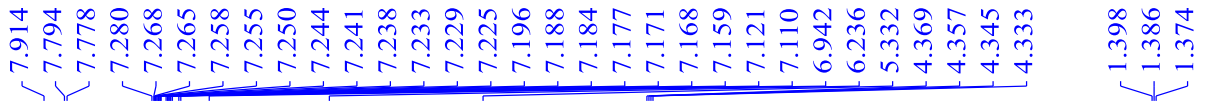
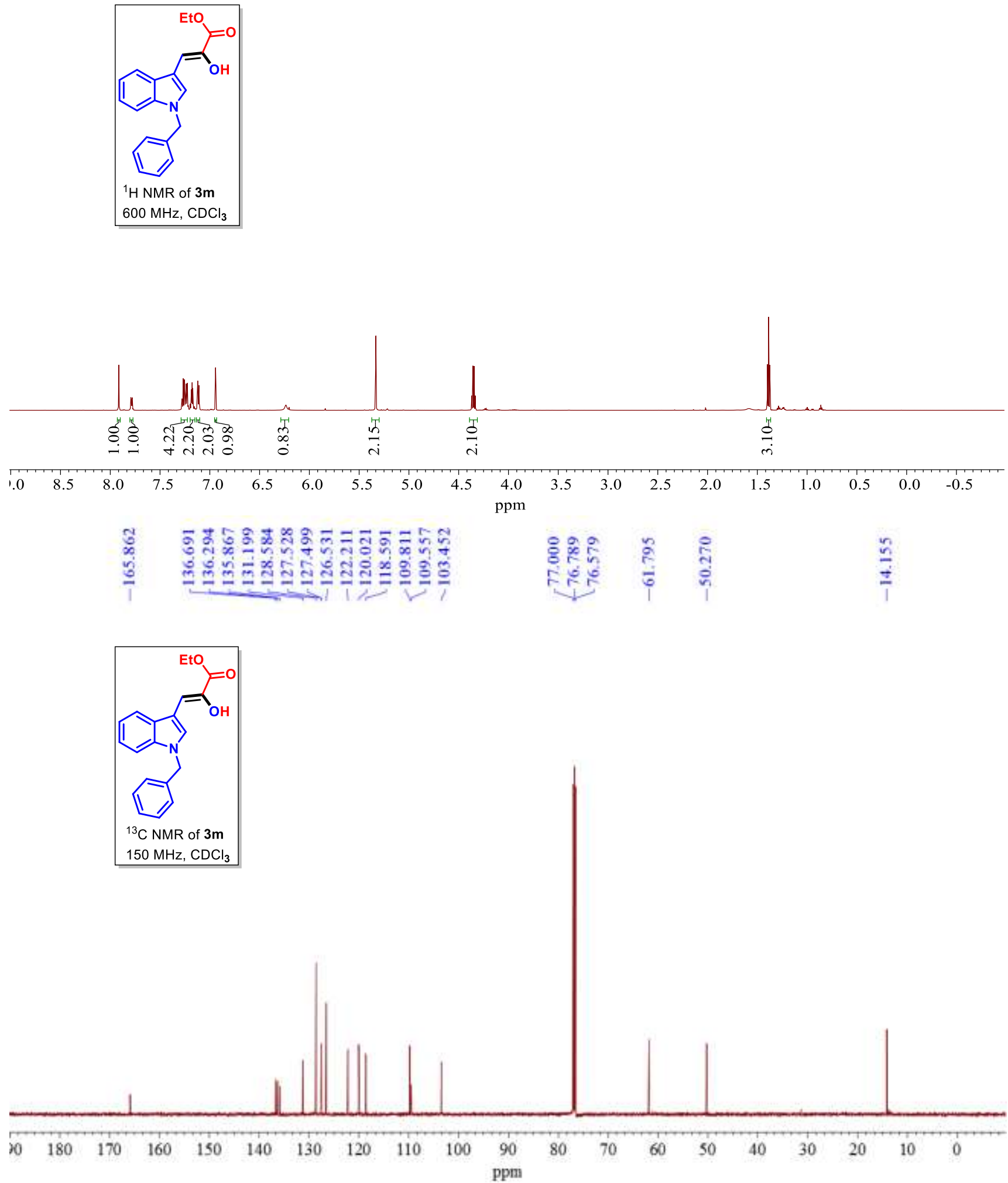

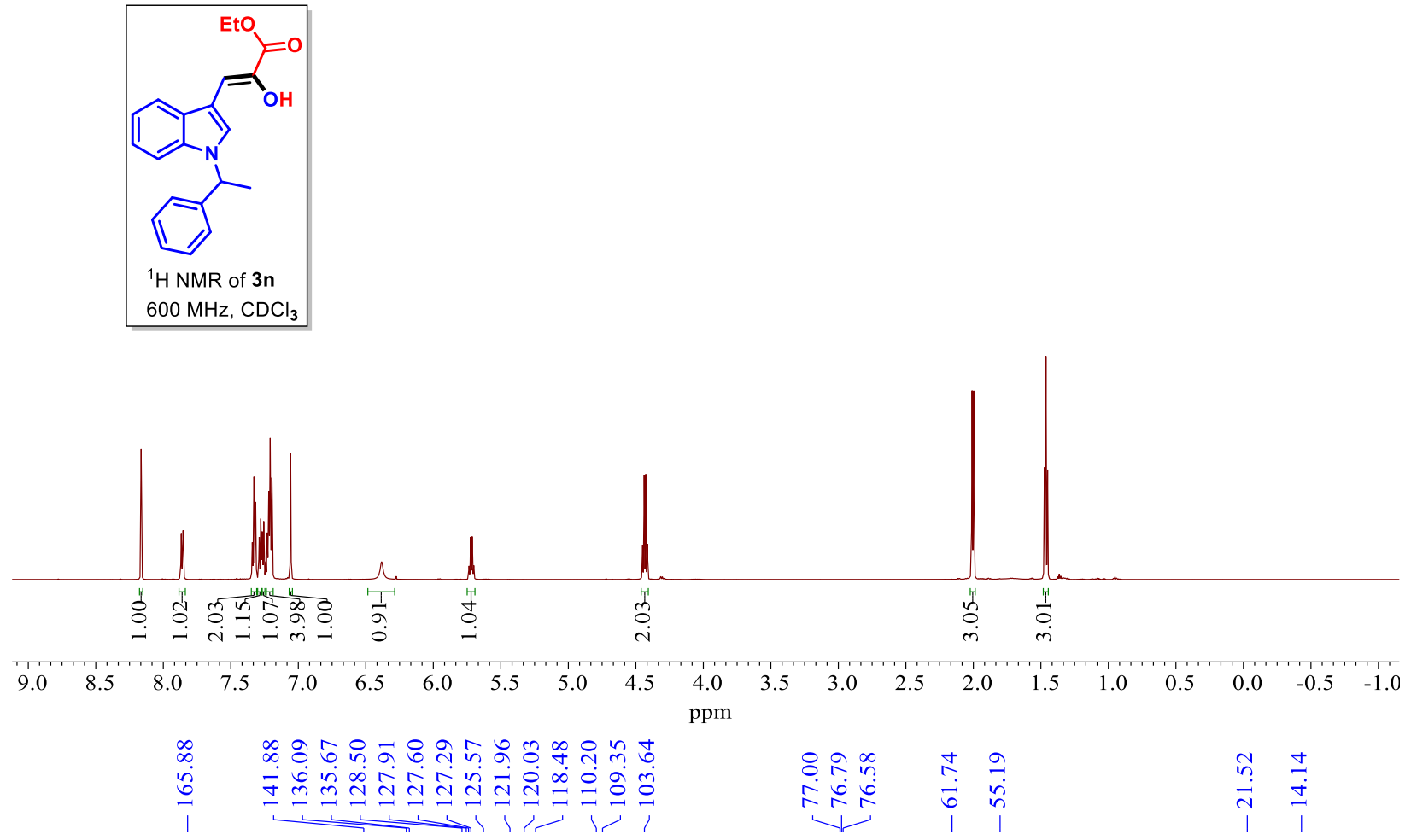

$\underset{i}{\stackrel{\Xi}{n}} \frac{ \pm}{ \pm}$

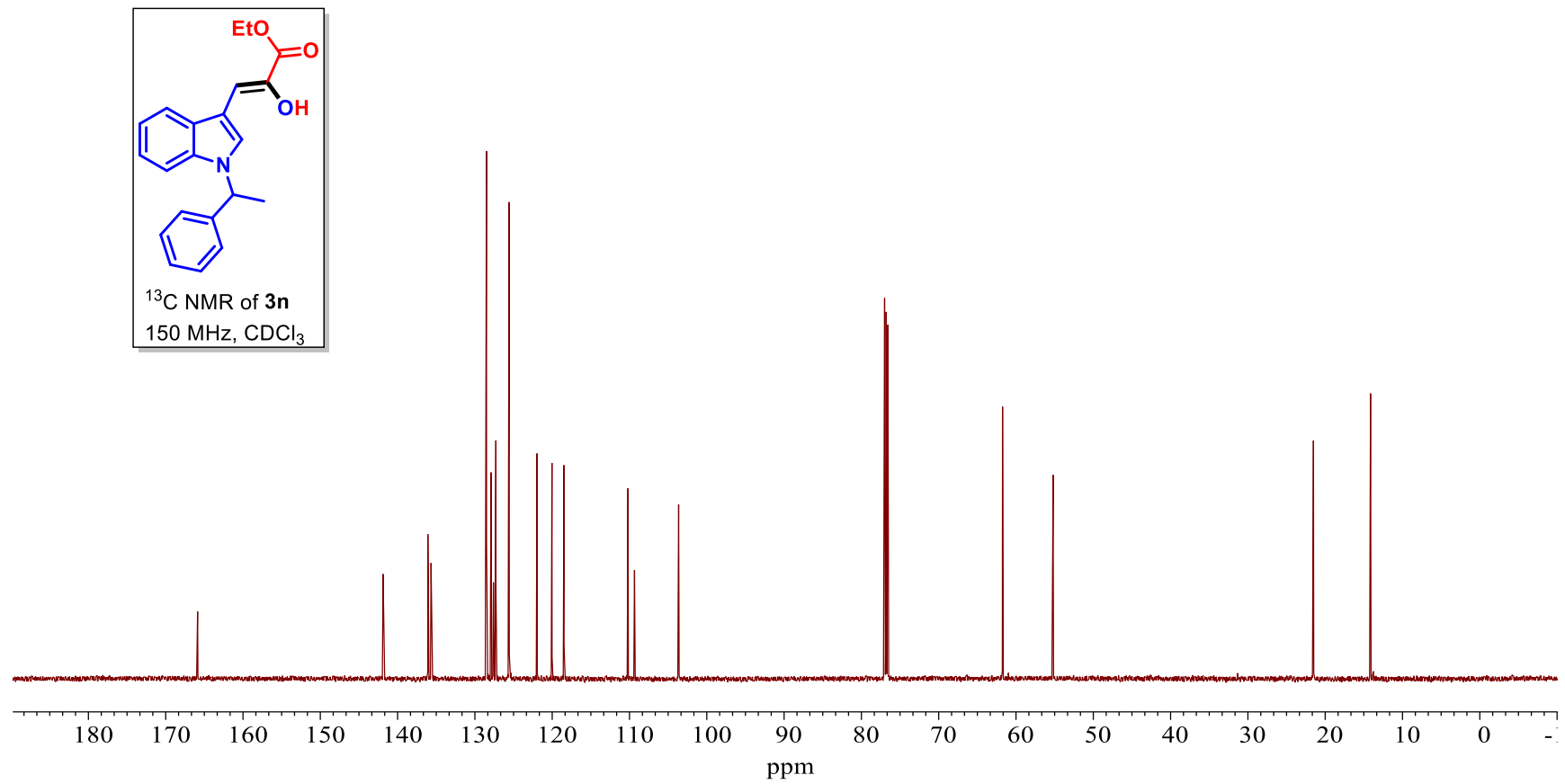




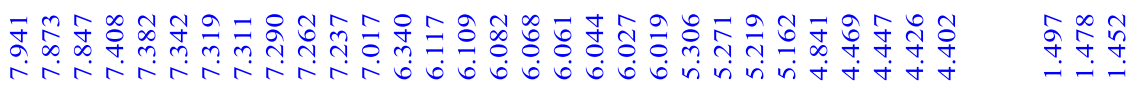

NATHANATH,
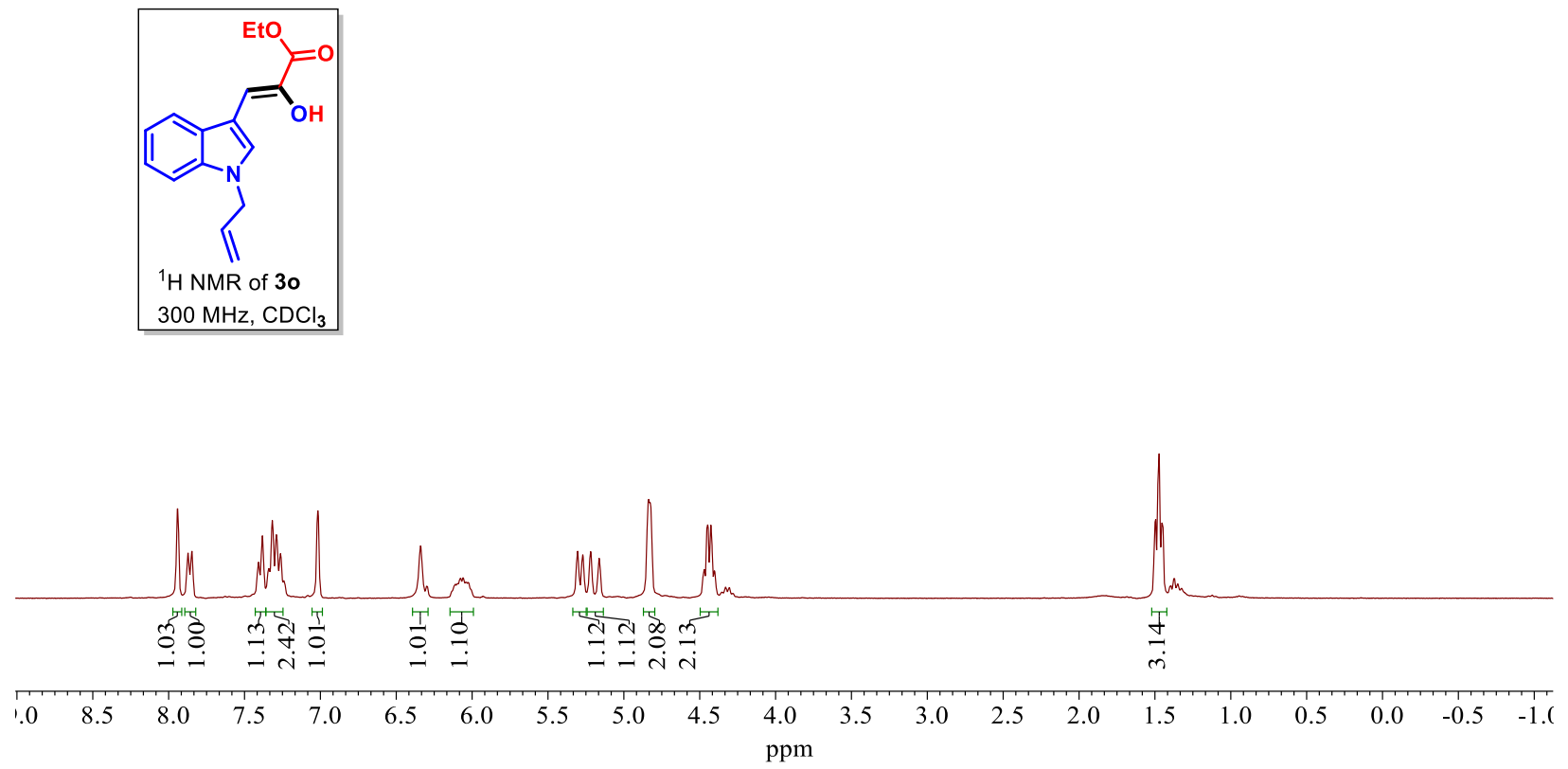

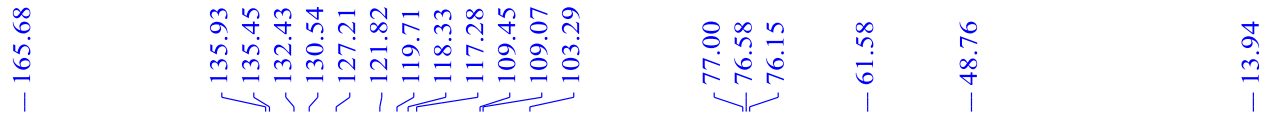

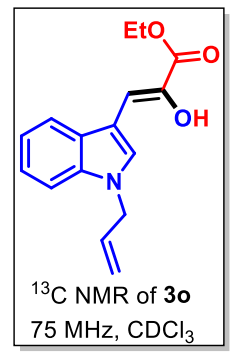

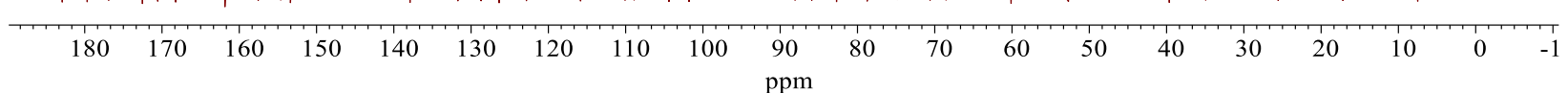



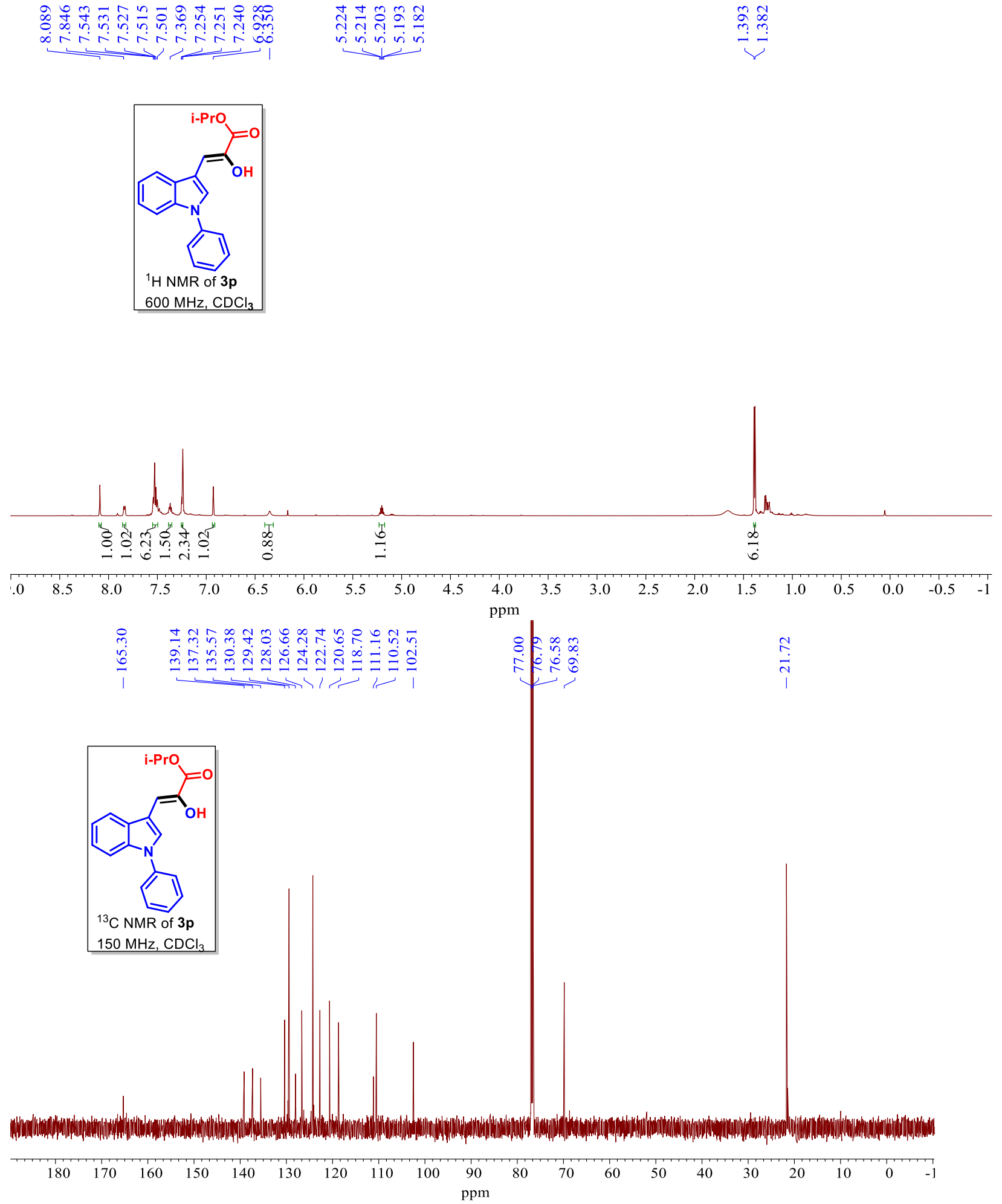

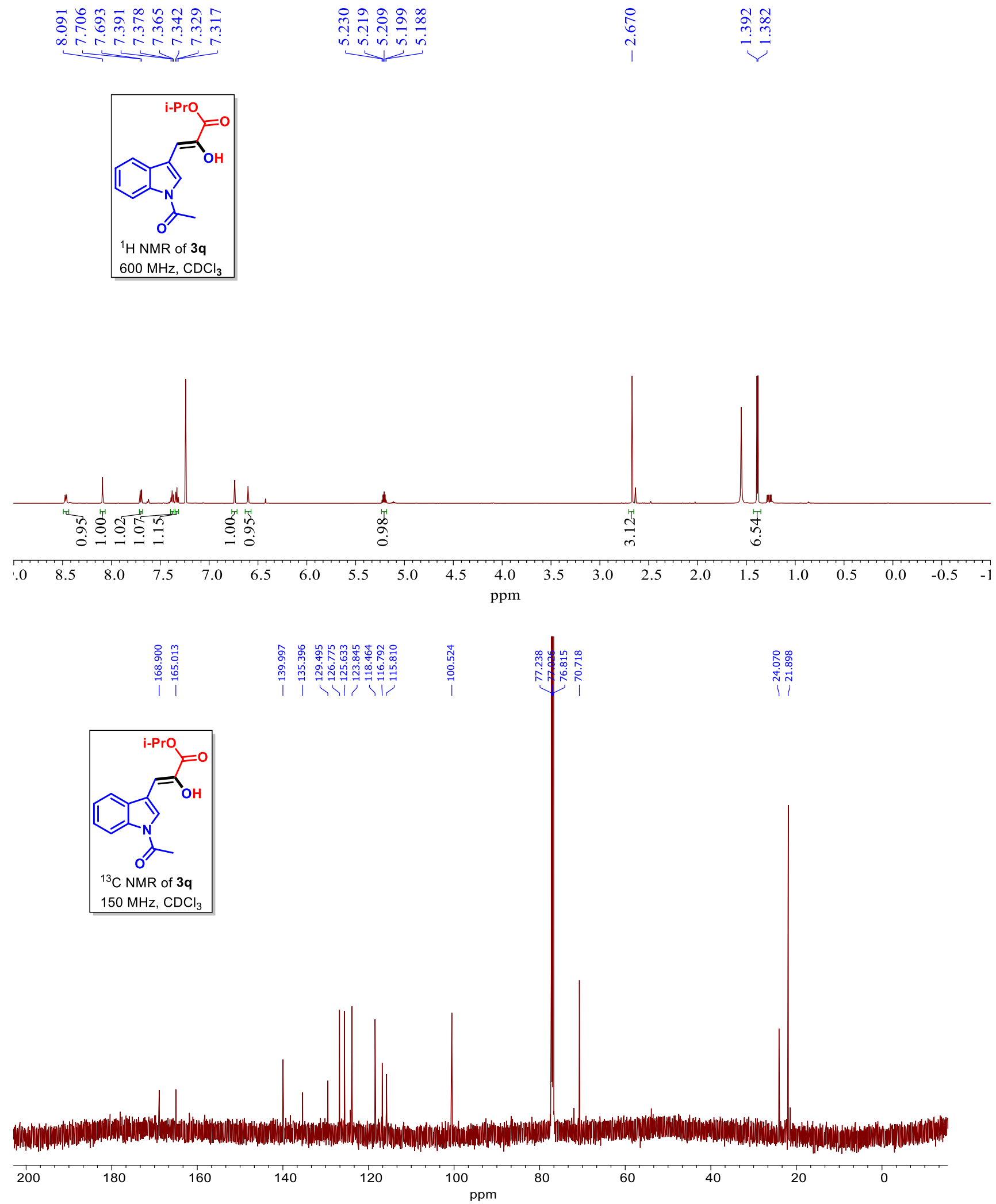


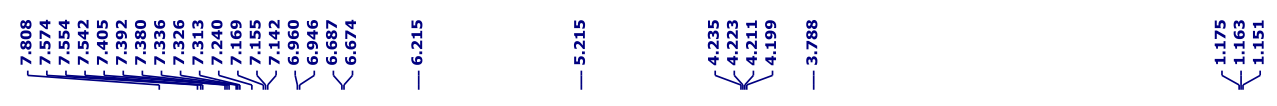
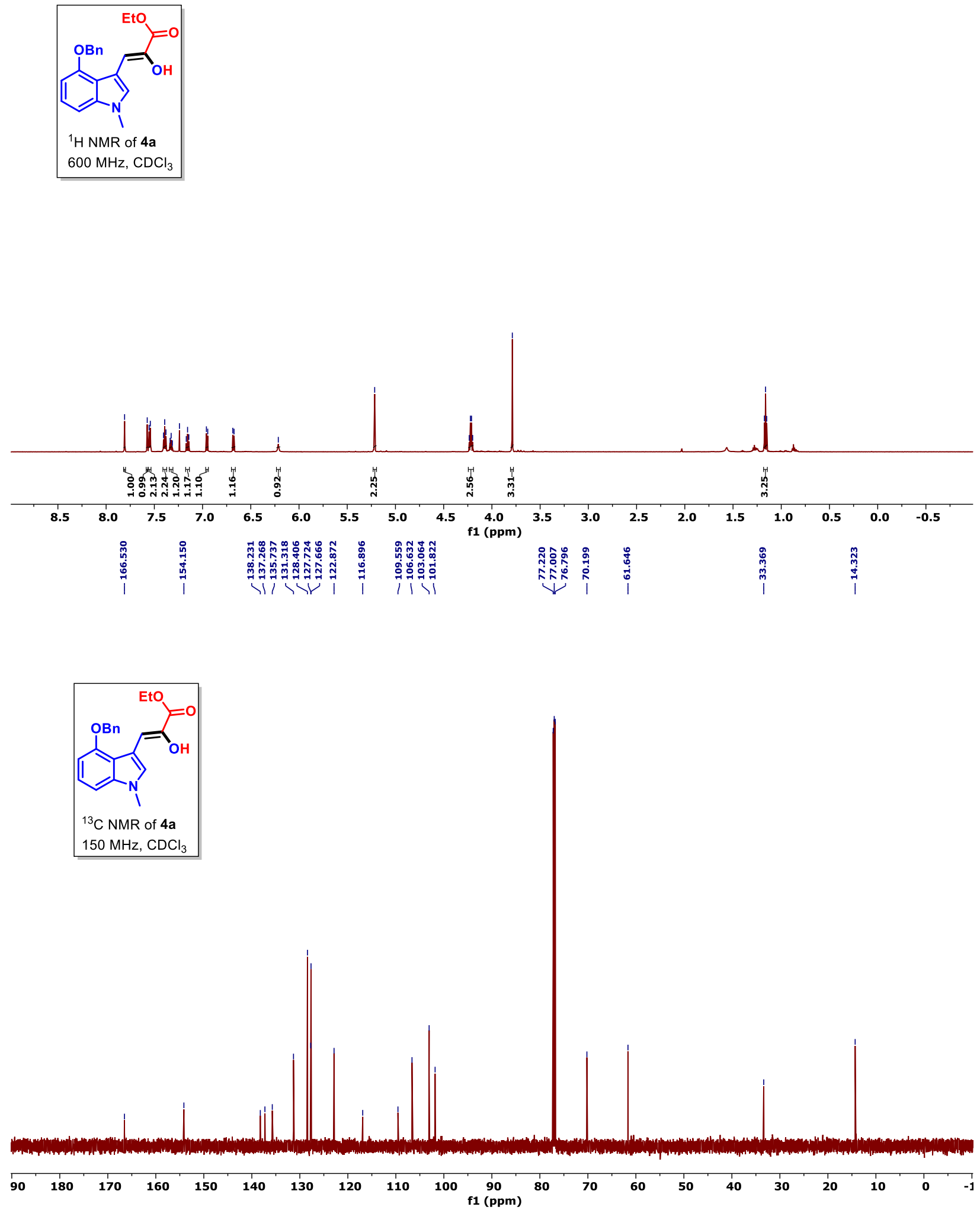


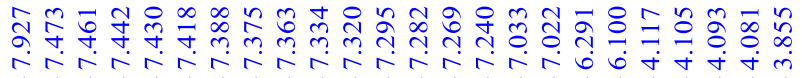
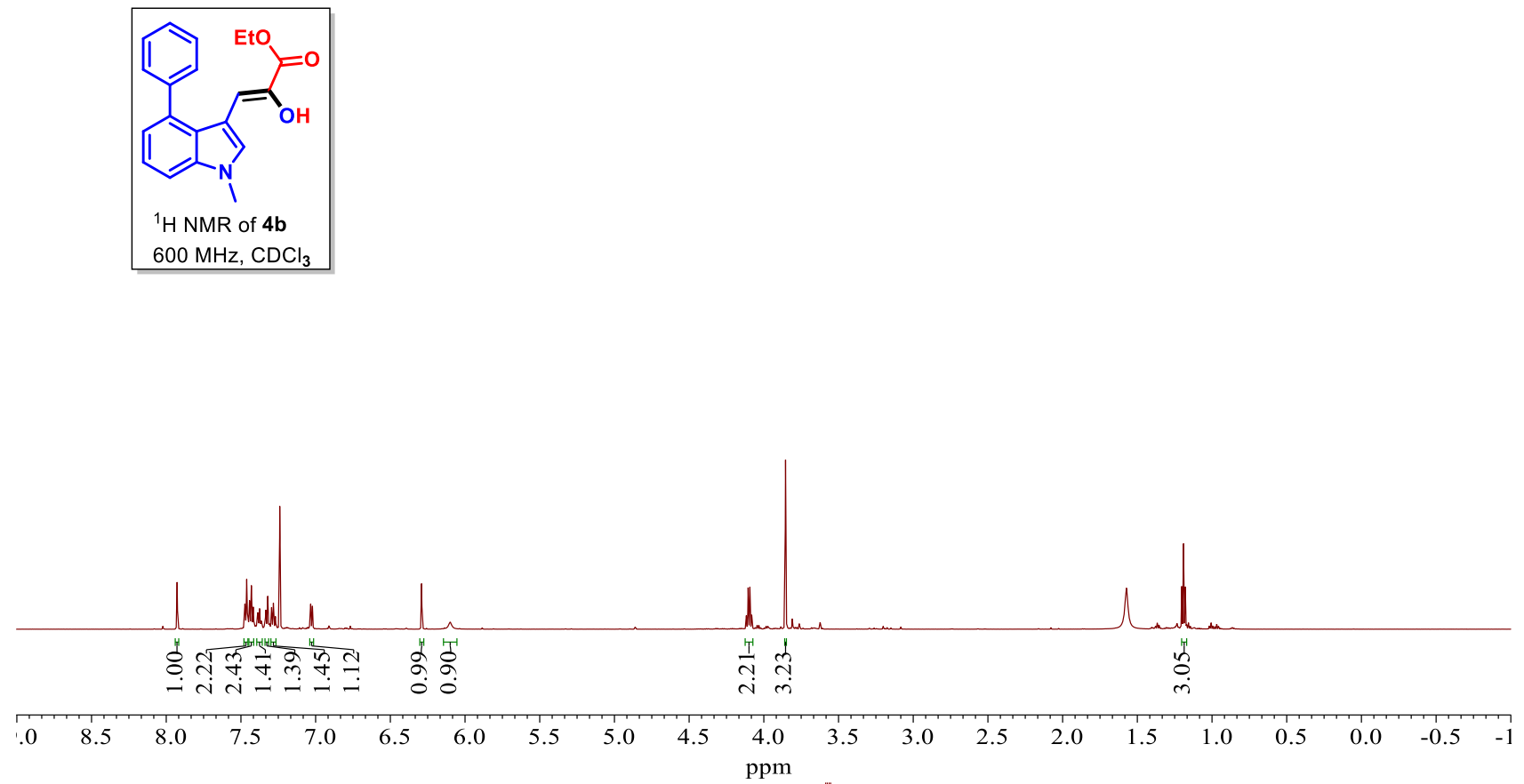

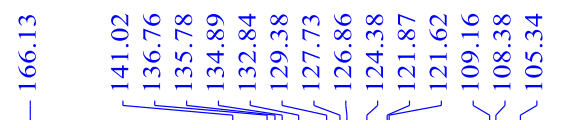

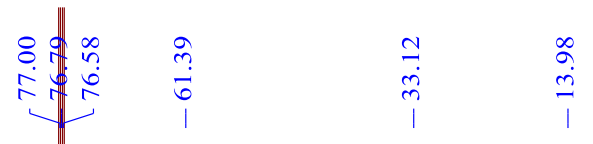

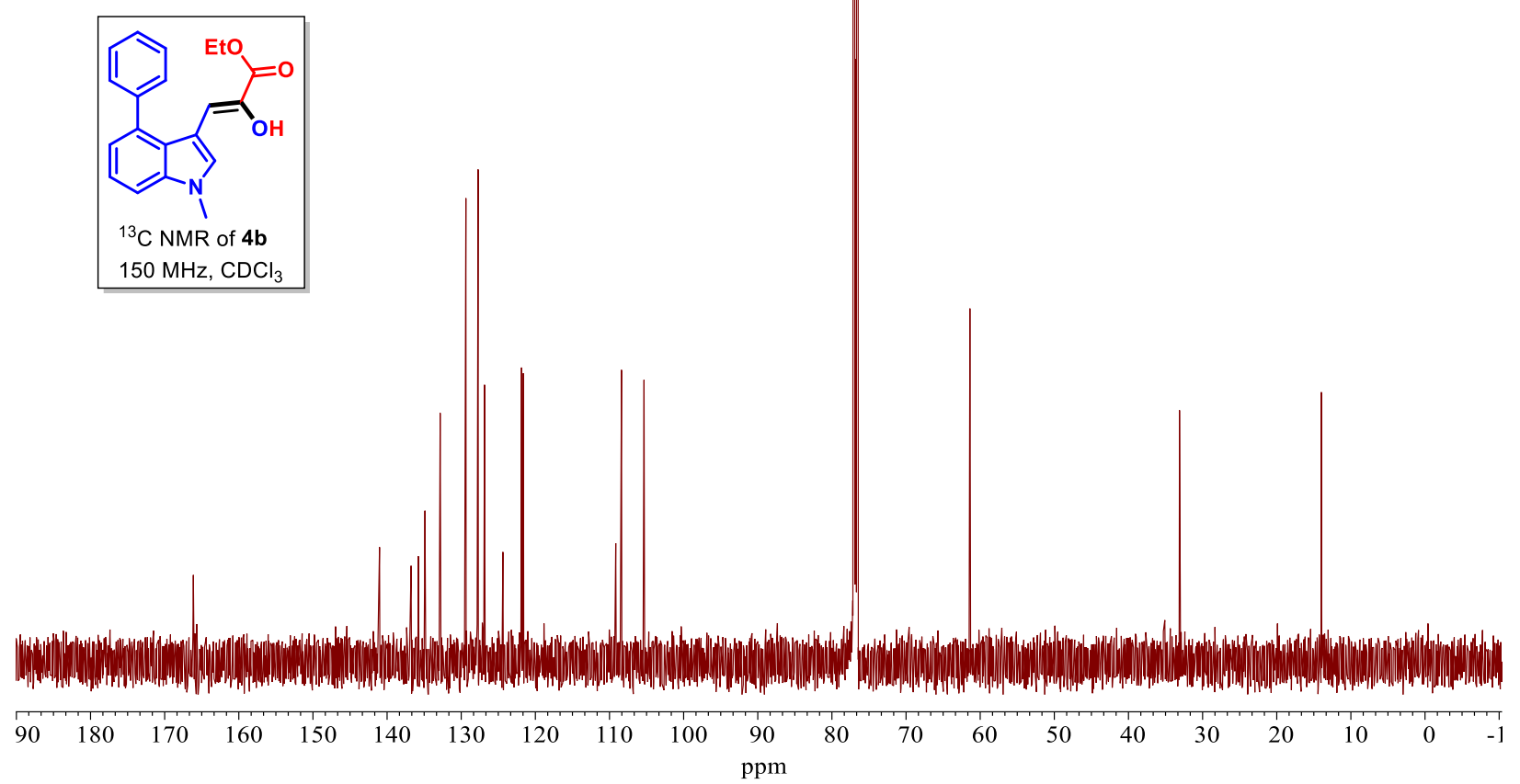




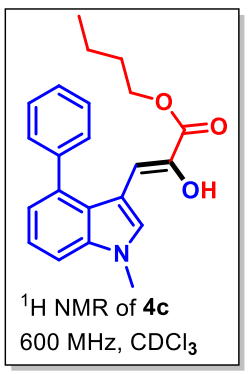

$600 \mathrm{MHz}^{\mathrm{CDCl}} \mathrm{C}_{3}$

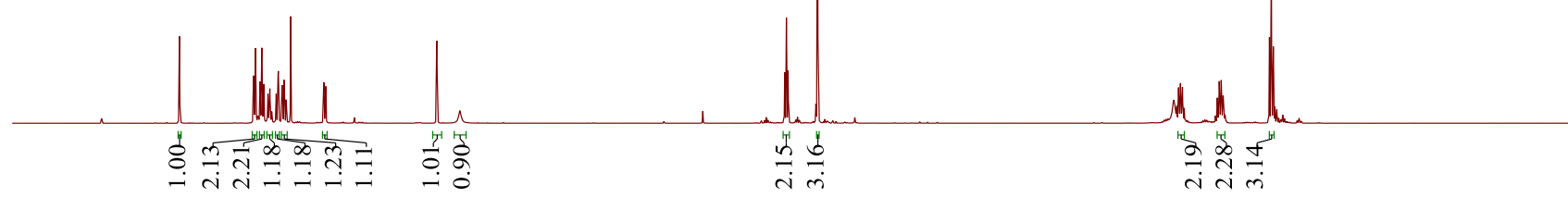

\begin{tabular}{lllllllllllllllllllllll}
\hline .0 & 8.5 & 8.0 & 7.5 & 7.0 & 6.5 & 6.0 & 5.5 & 5.0 & 4.5 & 4.0 & 3.5 & 3.0 & 2.5 & 2.0 & 1.5 & 1.0 & 0.5 & 0.0 & -0.5 &
\end{tabular}

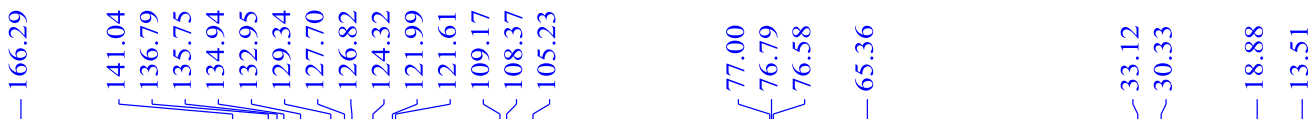
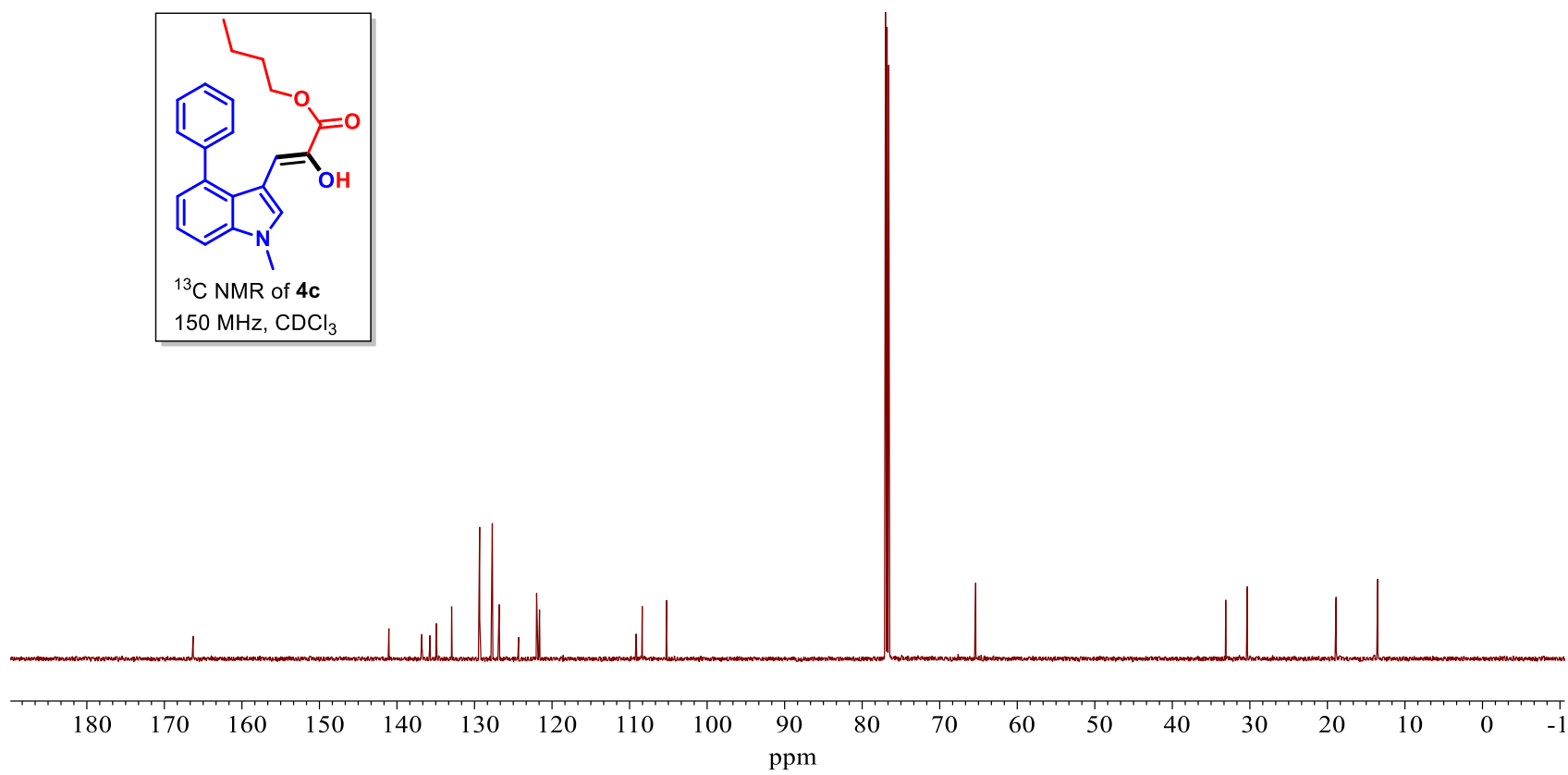


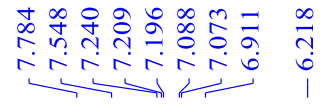

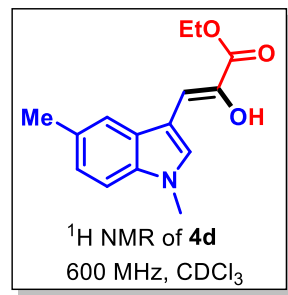

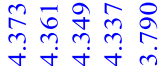

舟

$\underset{\substack{0 \\ i}}{\stackrel{m}{a}}$

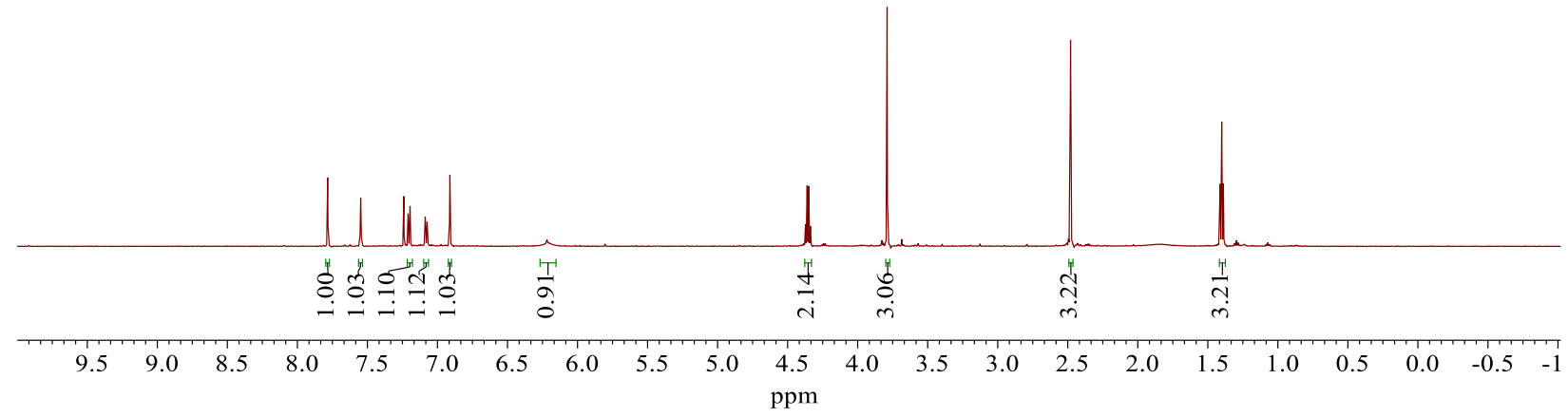

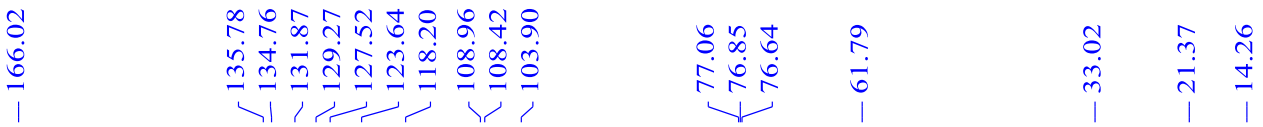
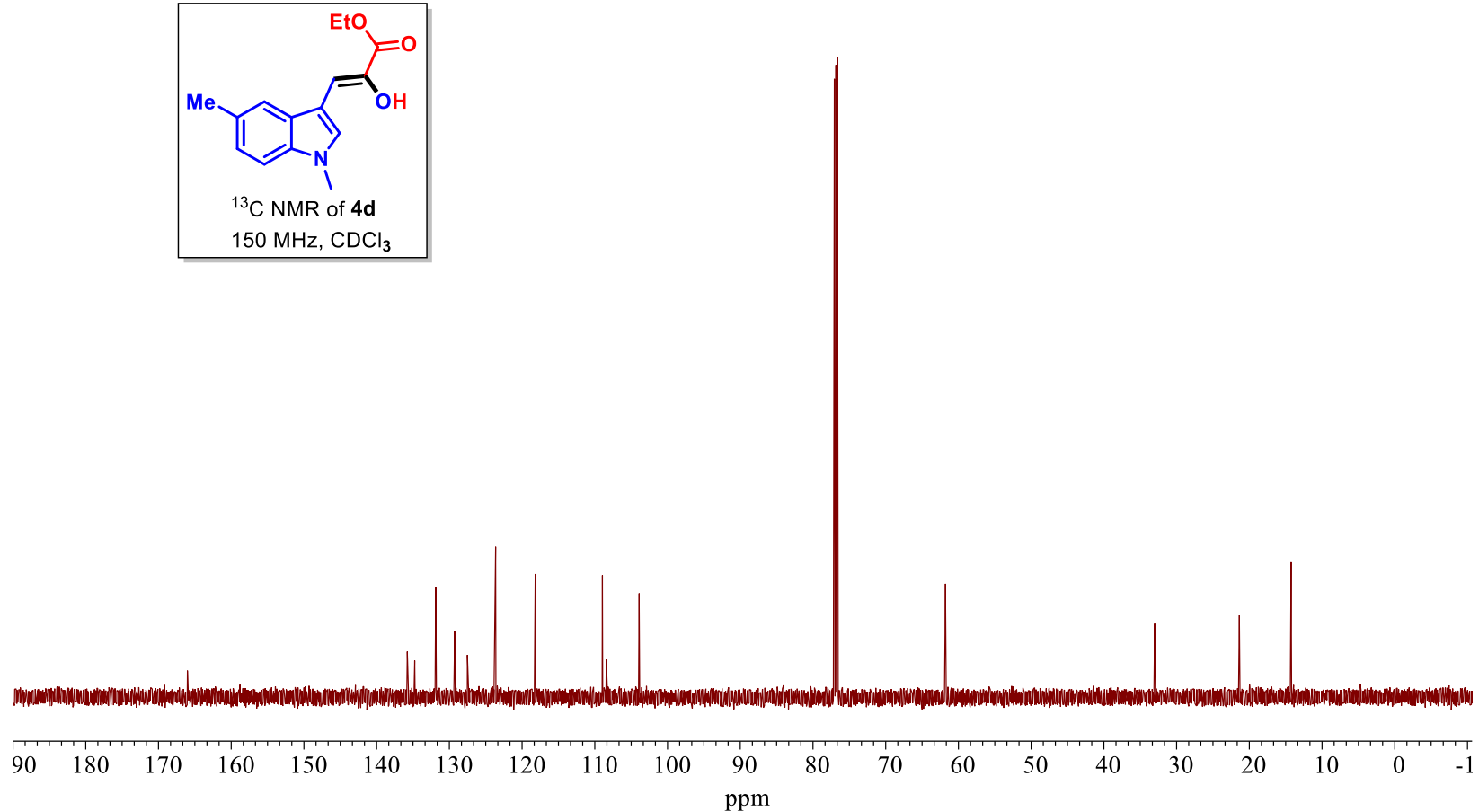

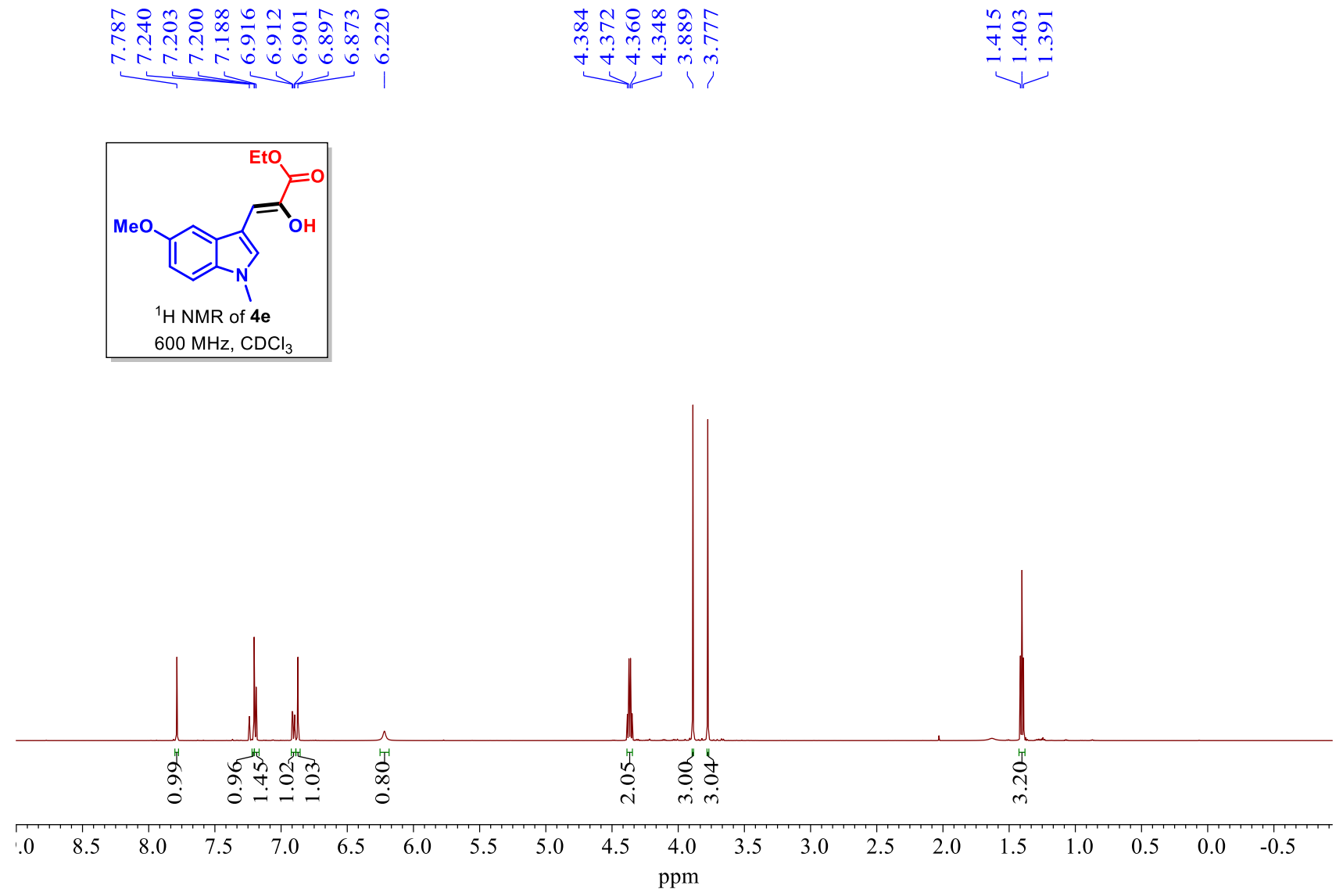

\begin{tabular}{|c|c|c|c|c|c|c|}
\hline 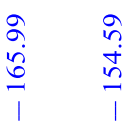 & 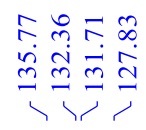 & 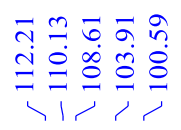 & 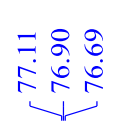 & $\begin{array}{l}m \\
\infty \\
\dot{b} \\
1\end{array}$ & $\begin{array}{l}+ \\
\infty \\
n \\
n \\
1\end{array}$ & $\stackrel{b}{m}$ \\
\hline
\end{tabular}
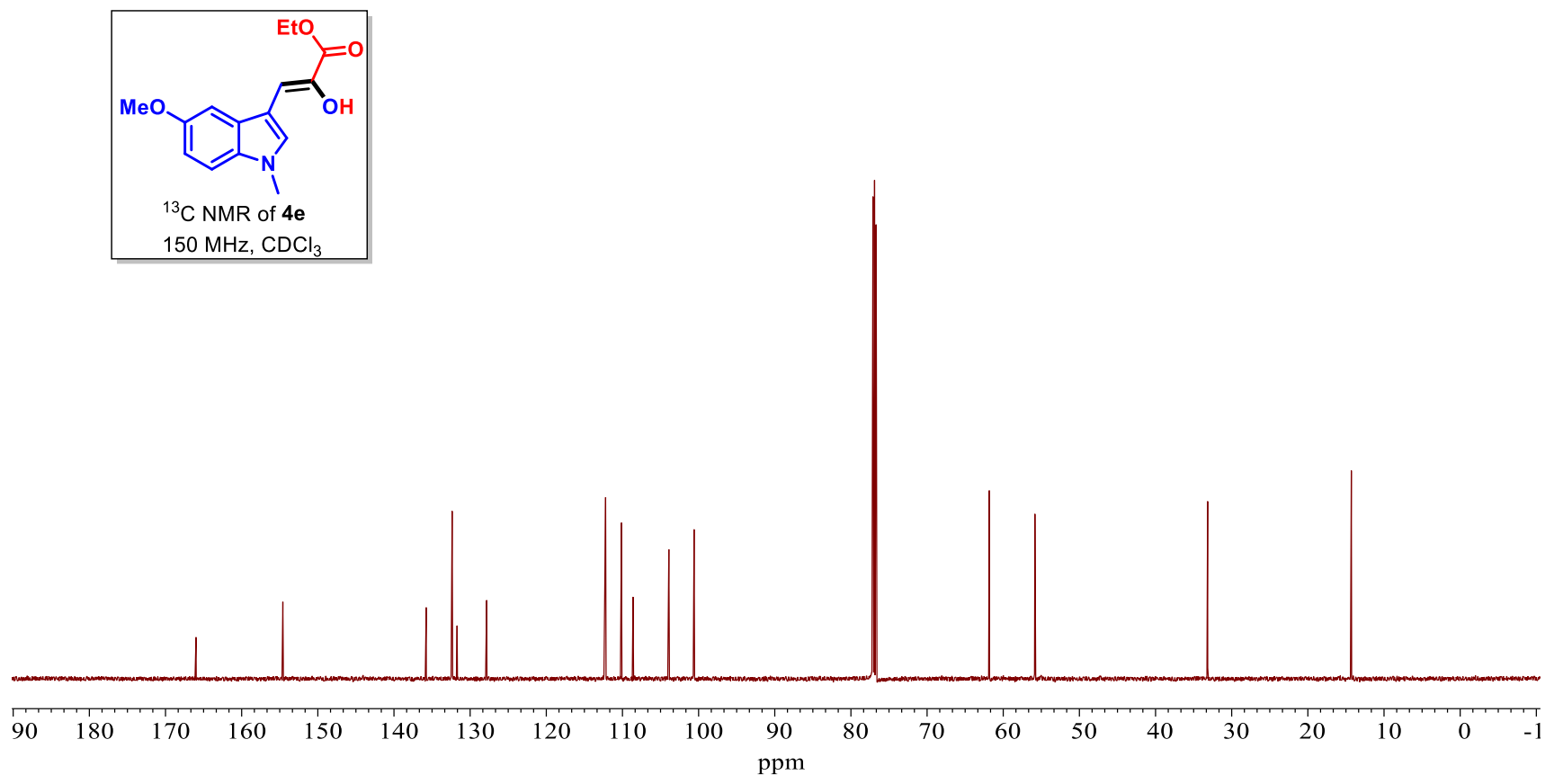


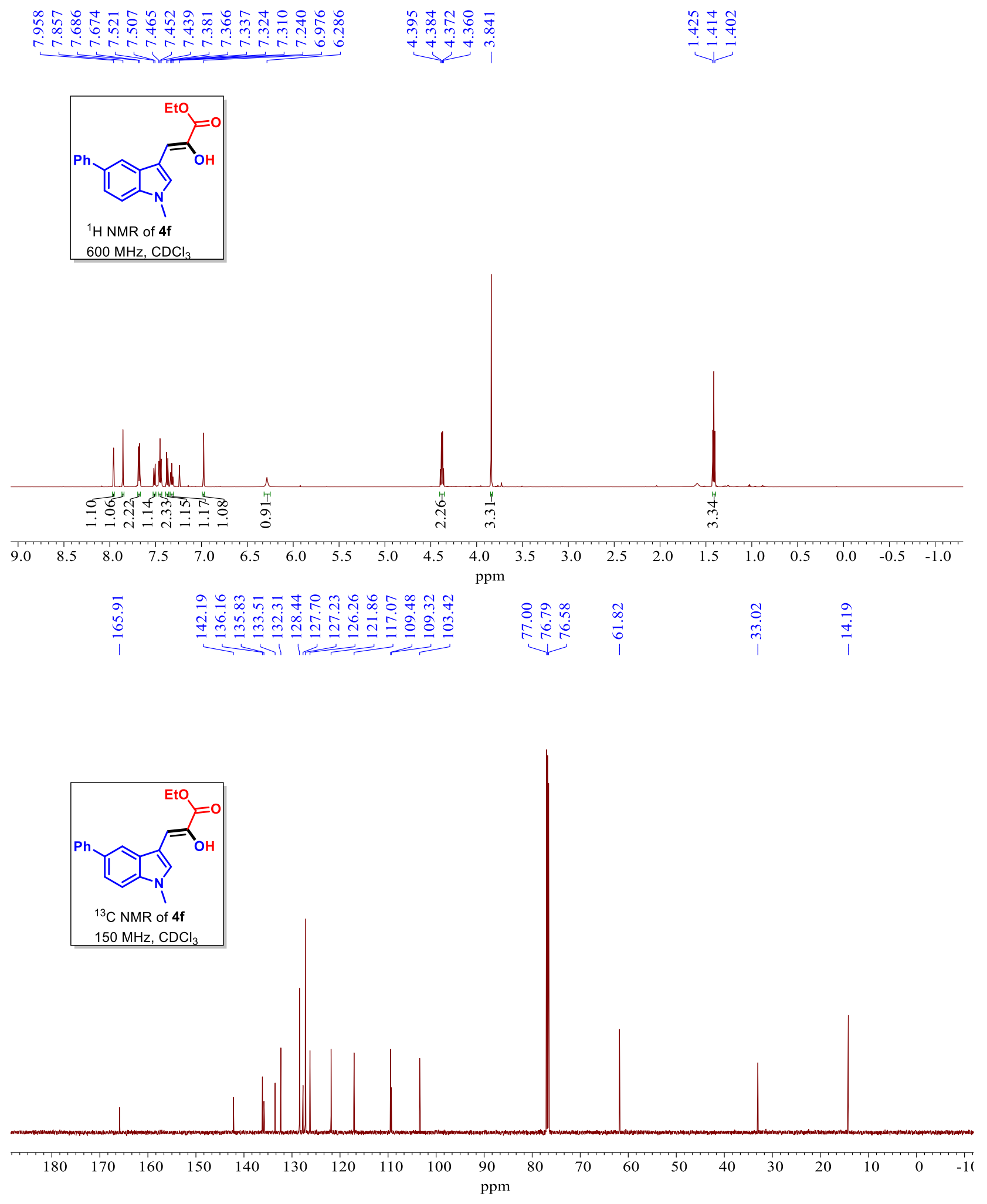



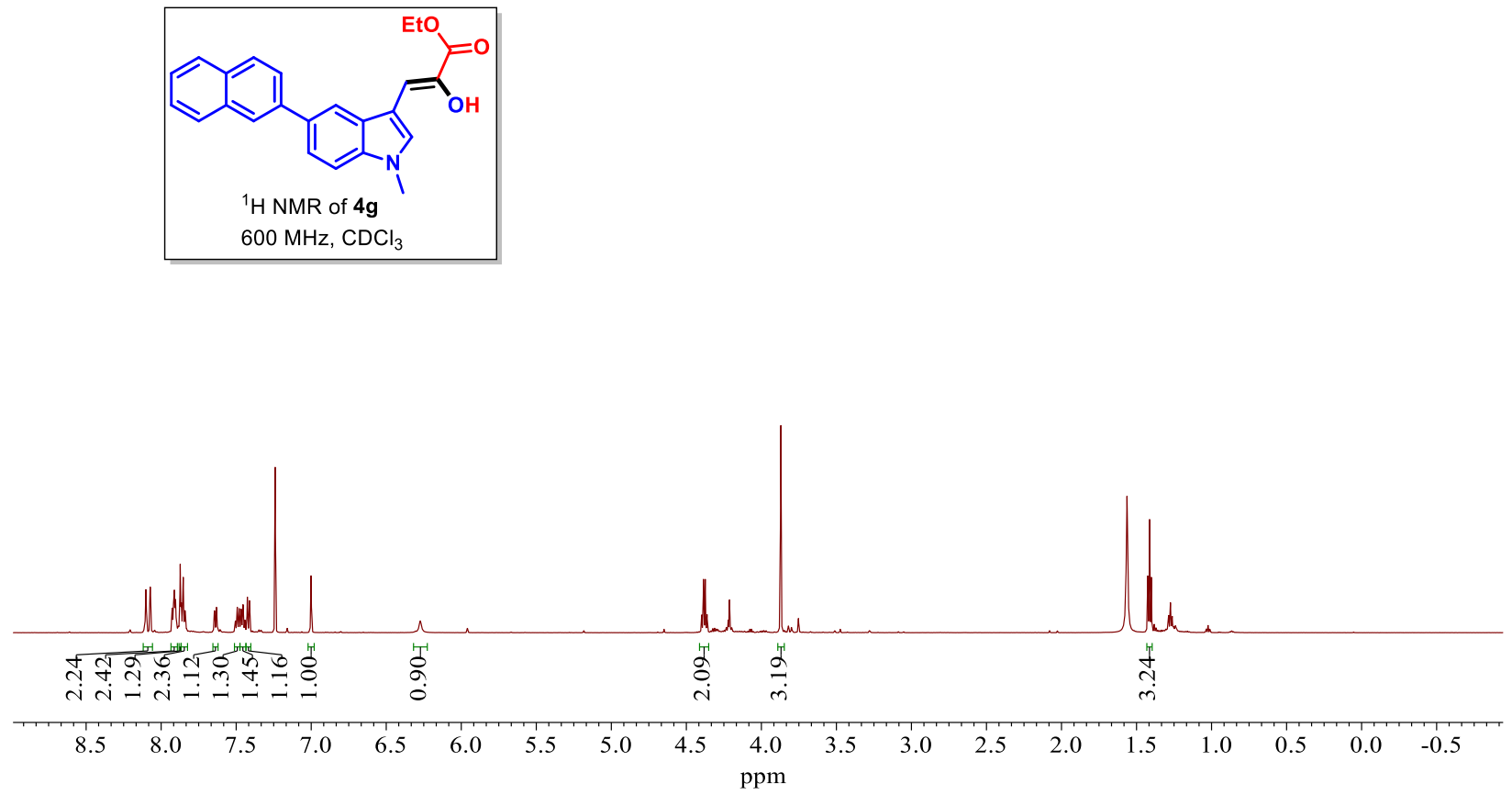

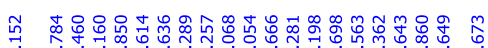

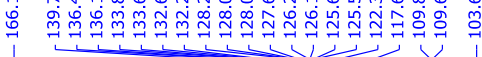
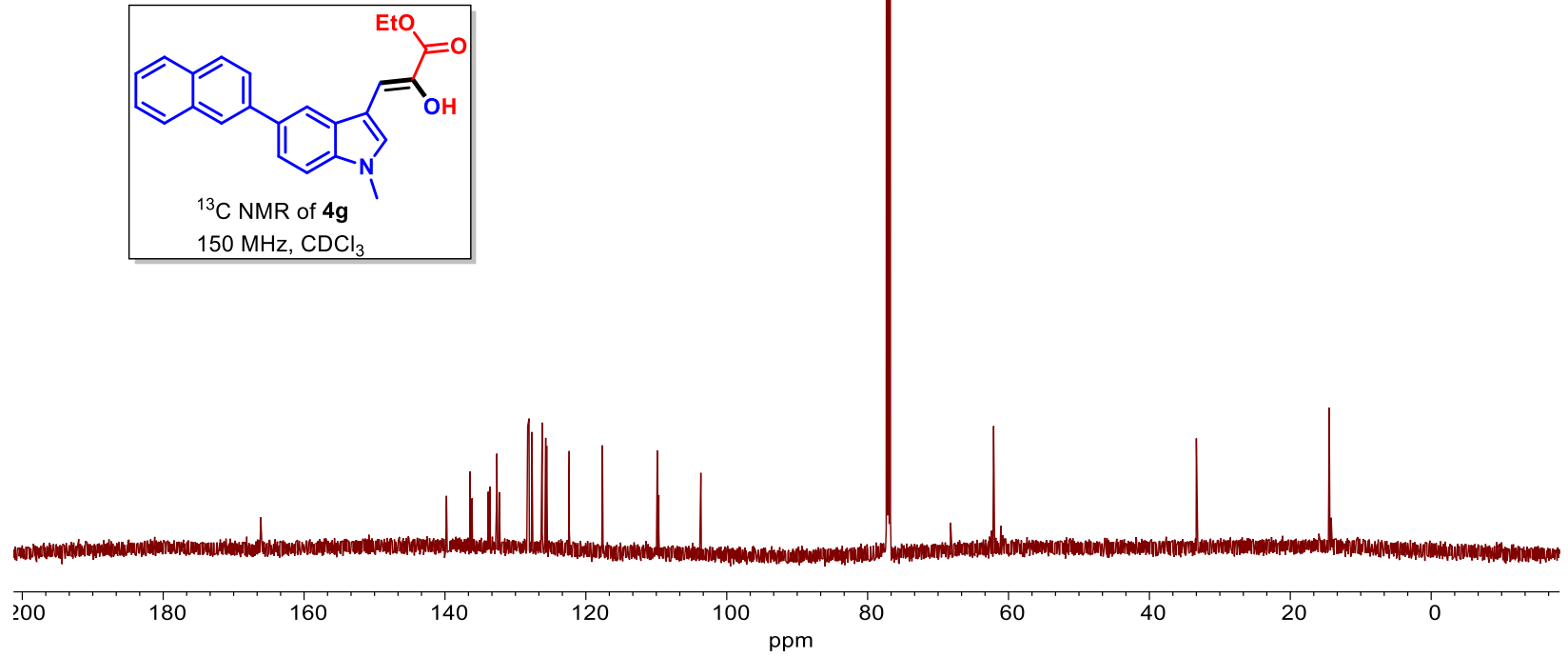


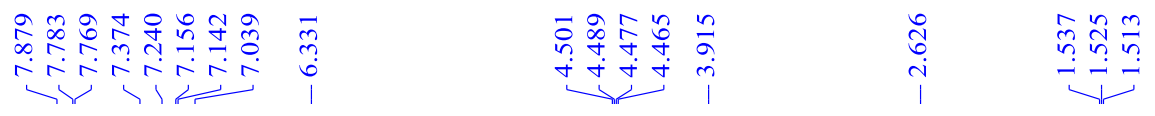
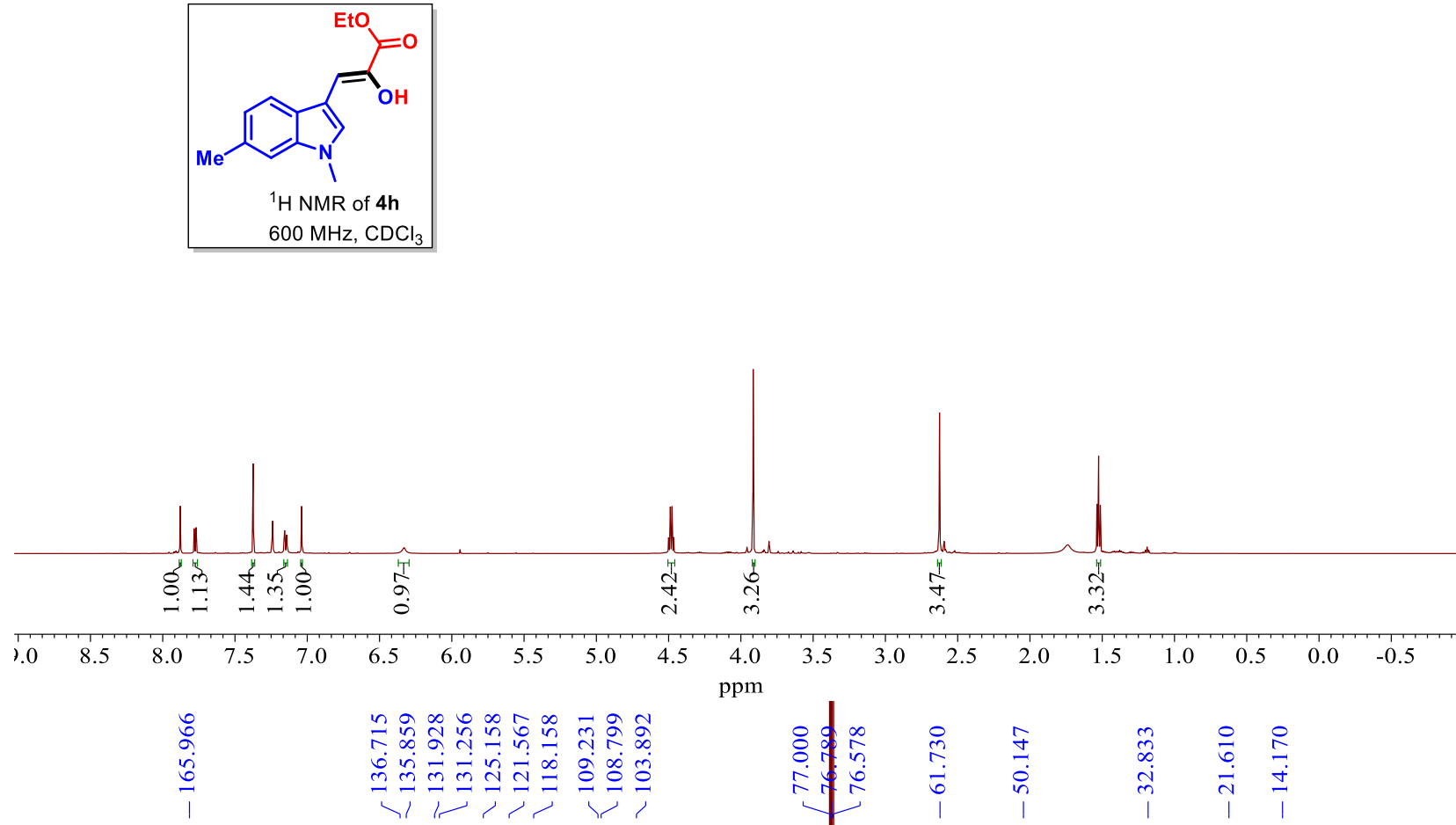

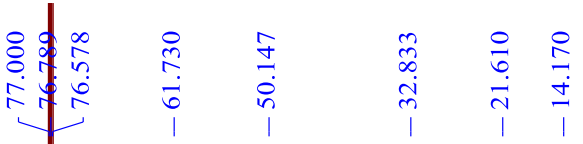

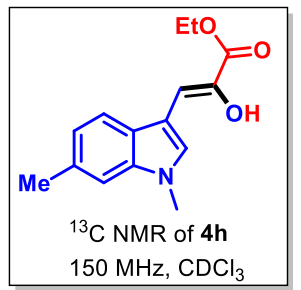

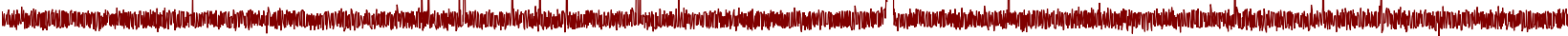

\begin{tabular}{lllllllllllllllllllllllll}
\hline 90 & 180 & 170 & 160 & 150 & 140 & 130 & 120 & 110 & 100 & 90 & 80 & 70 & 60 & 50 & 40 & 30 & 20 & 10 & 0 & -
\end{tabular}



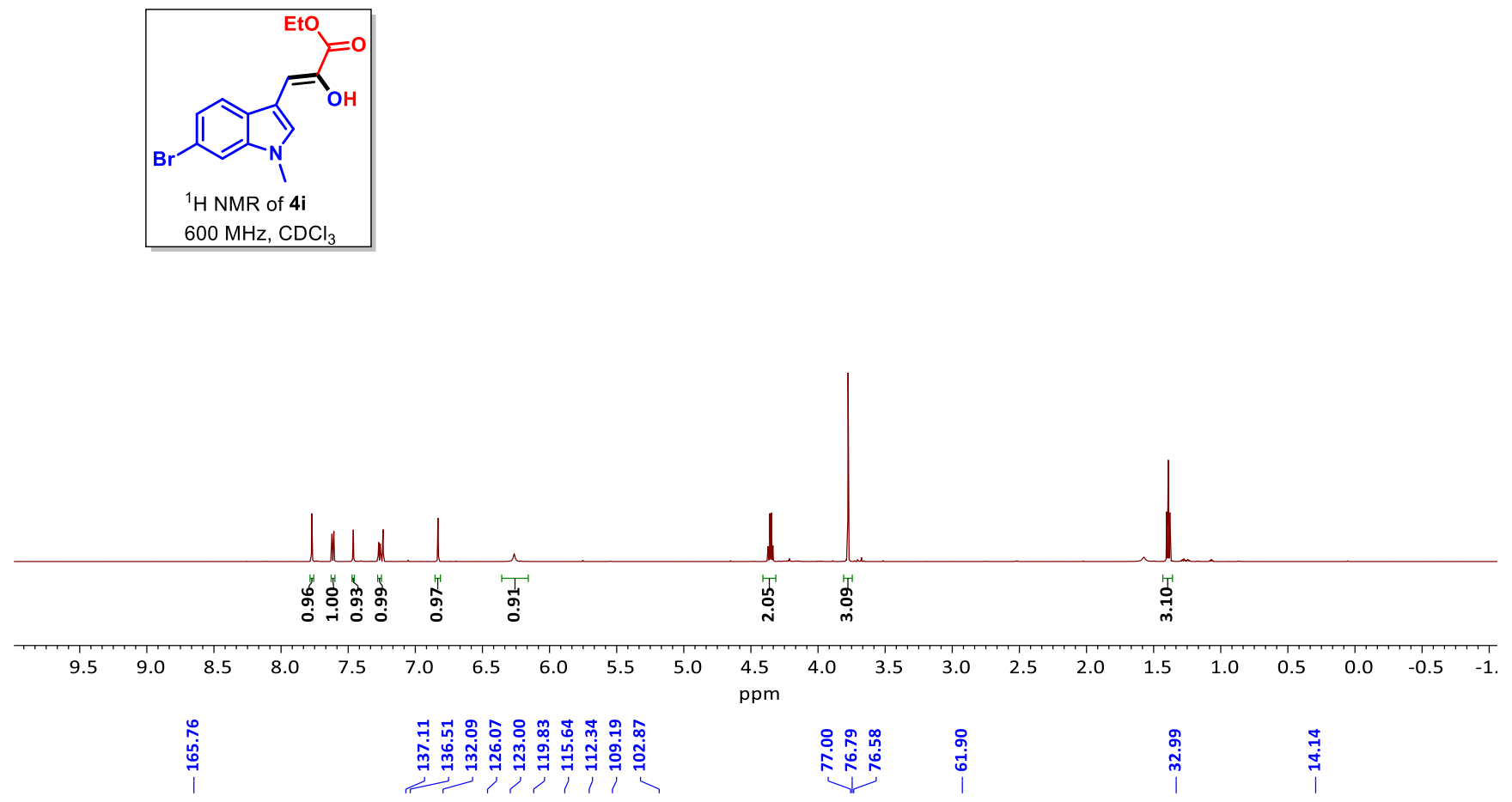

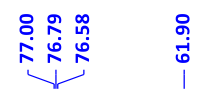

กั


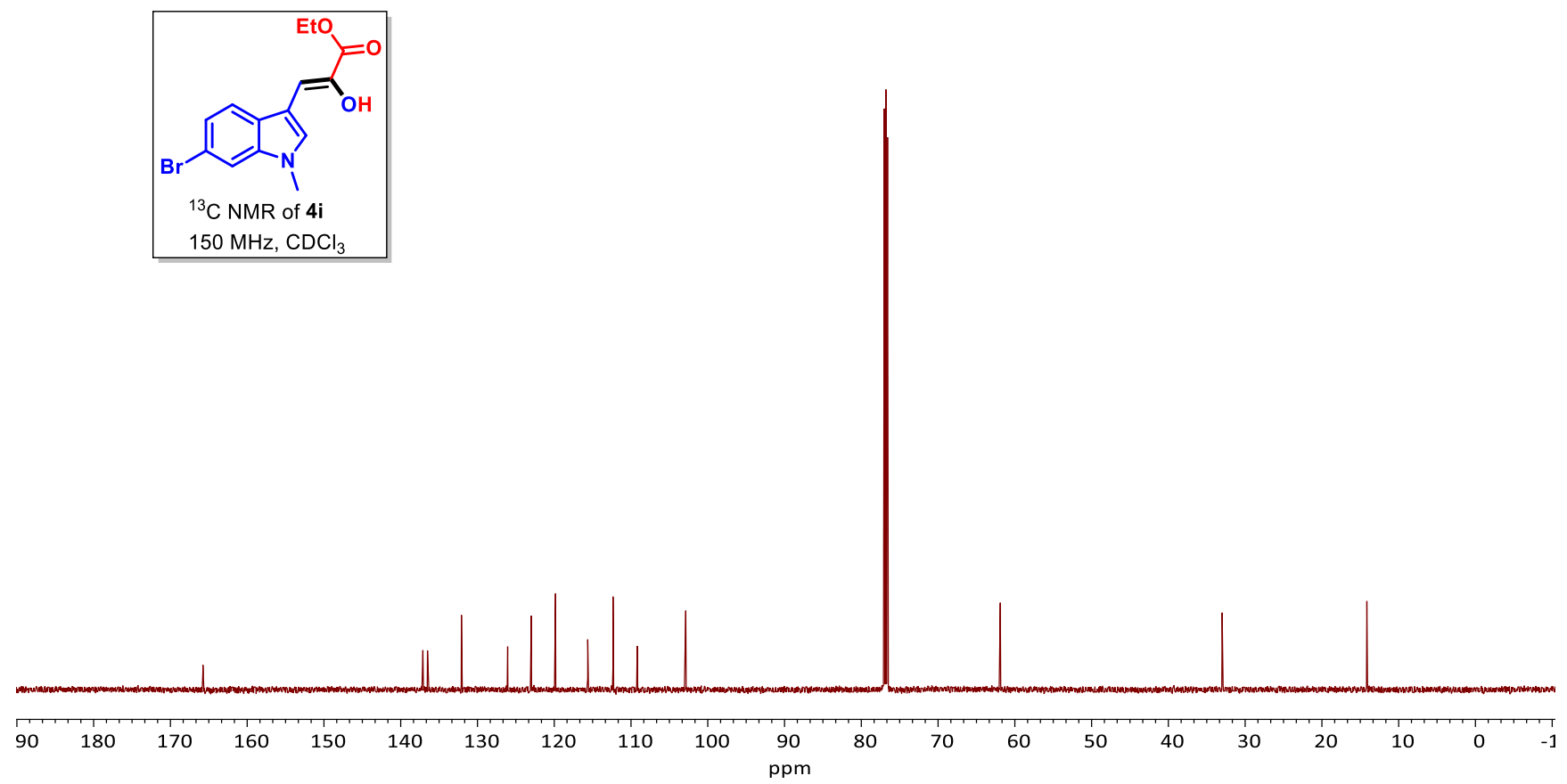

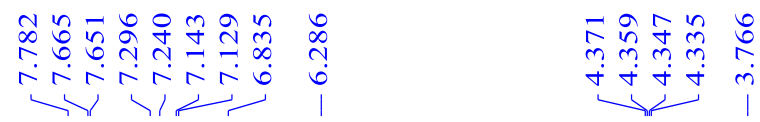

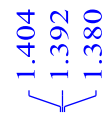
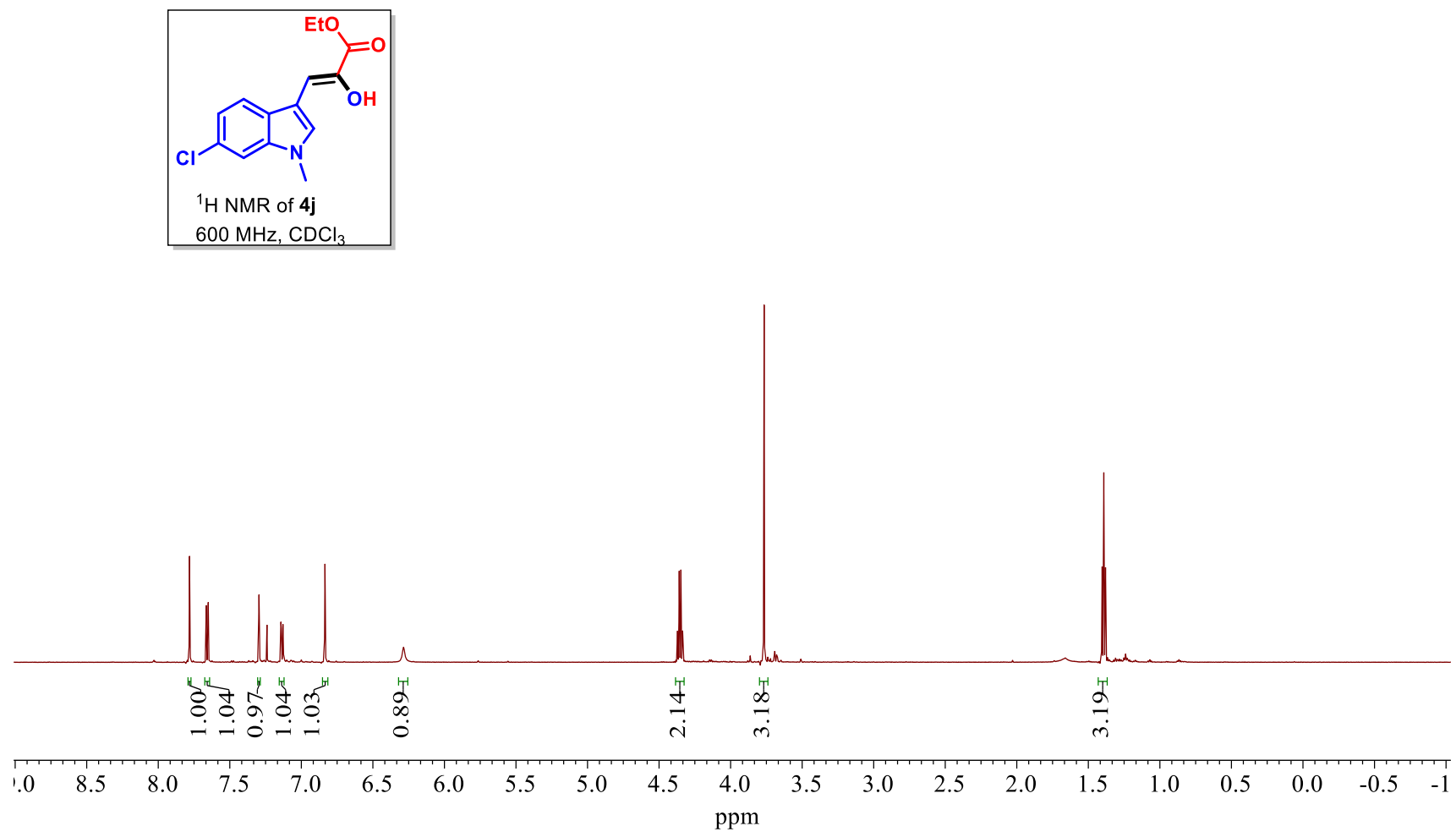

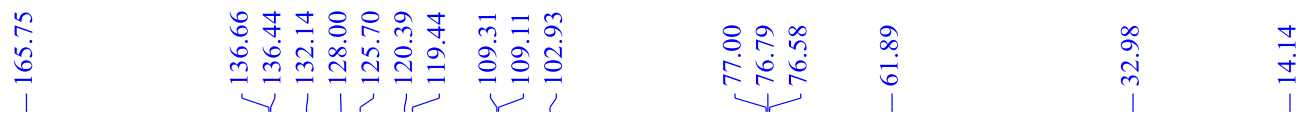
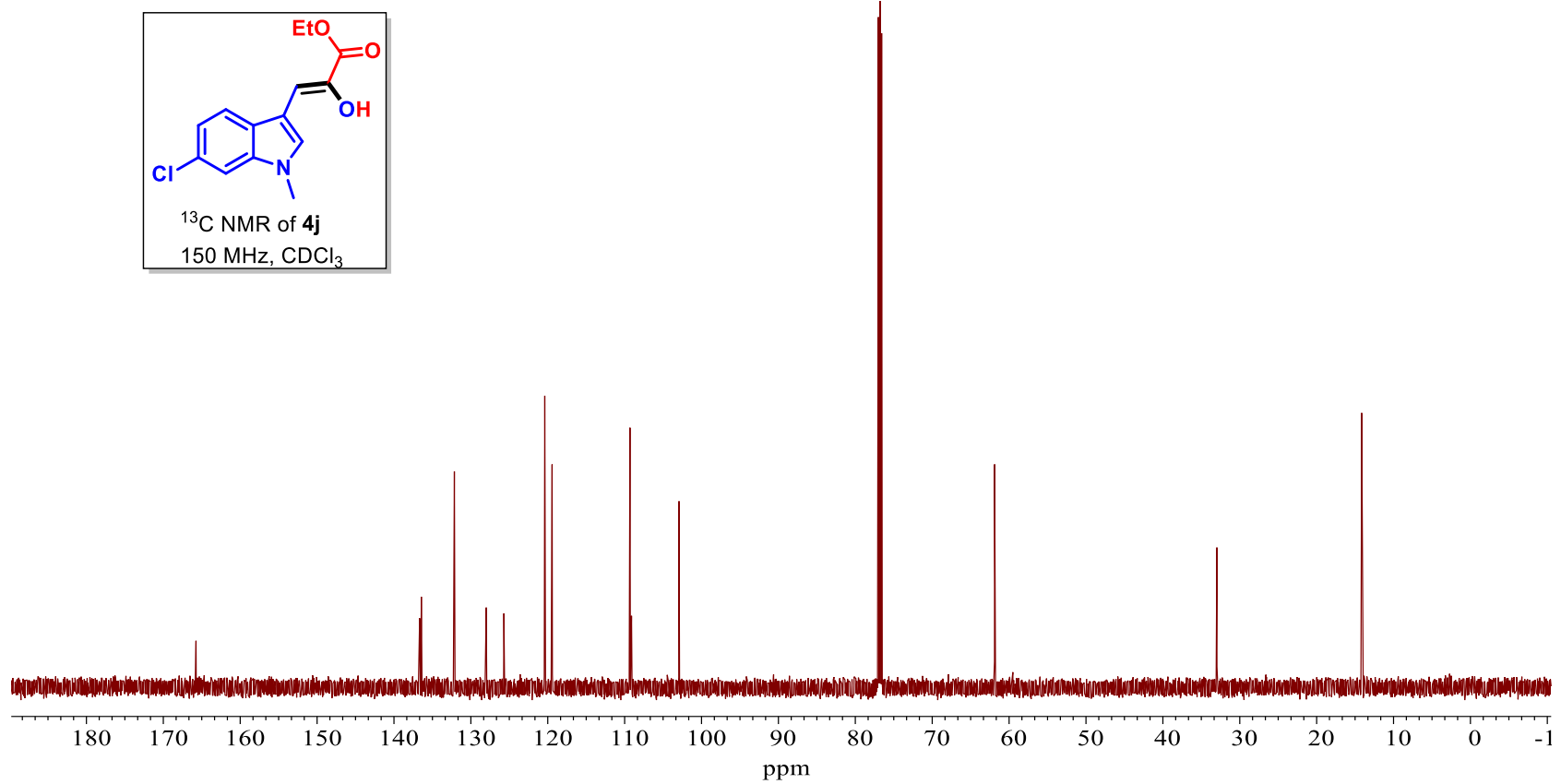

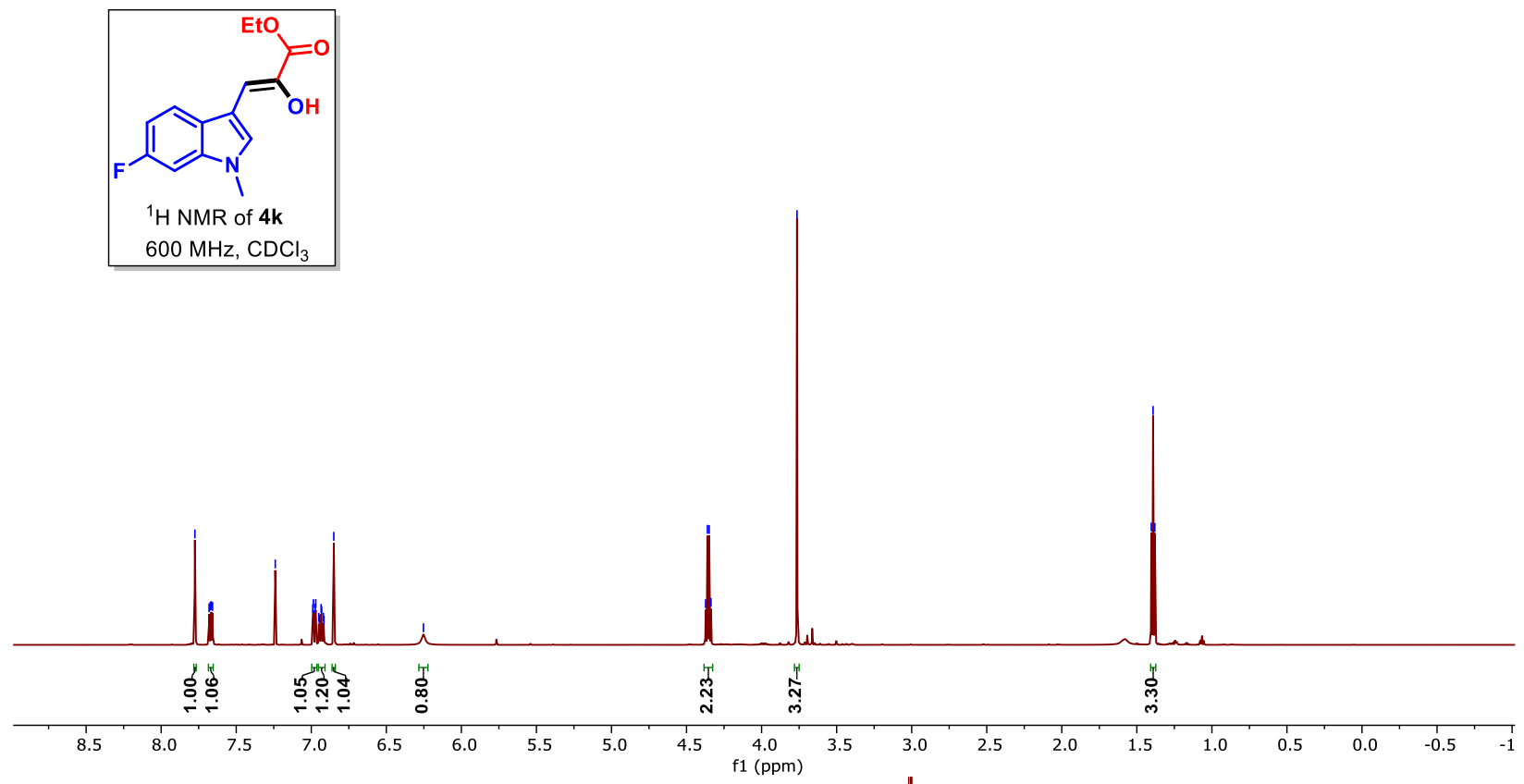

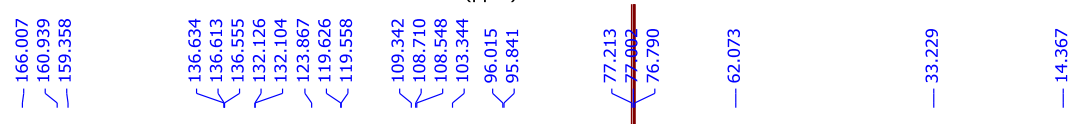

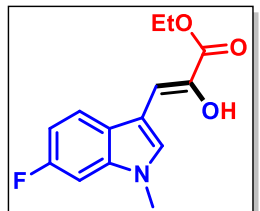

${ }^{13} \mathrm{C}$ NMR of $4 \mathbf{k}$ $150 \mathrm{MHz}, \mathrm{CDCl}_{3}$

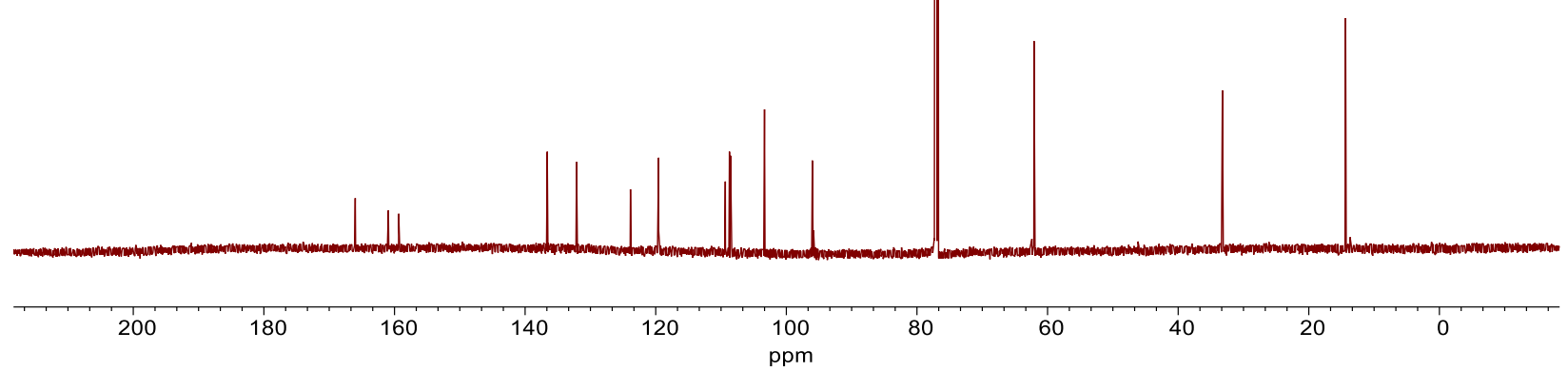



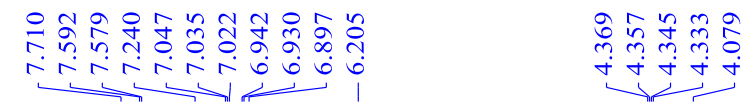

$\stackrel{n}{n}$ in
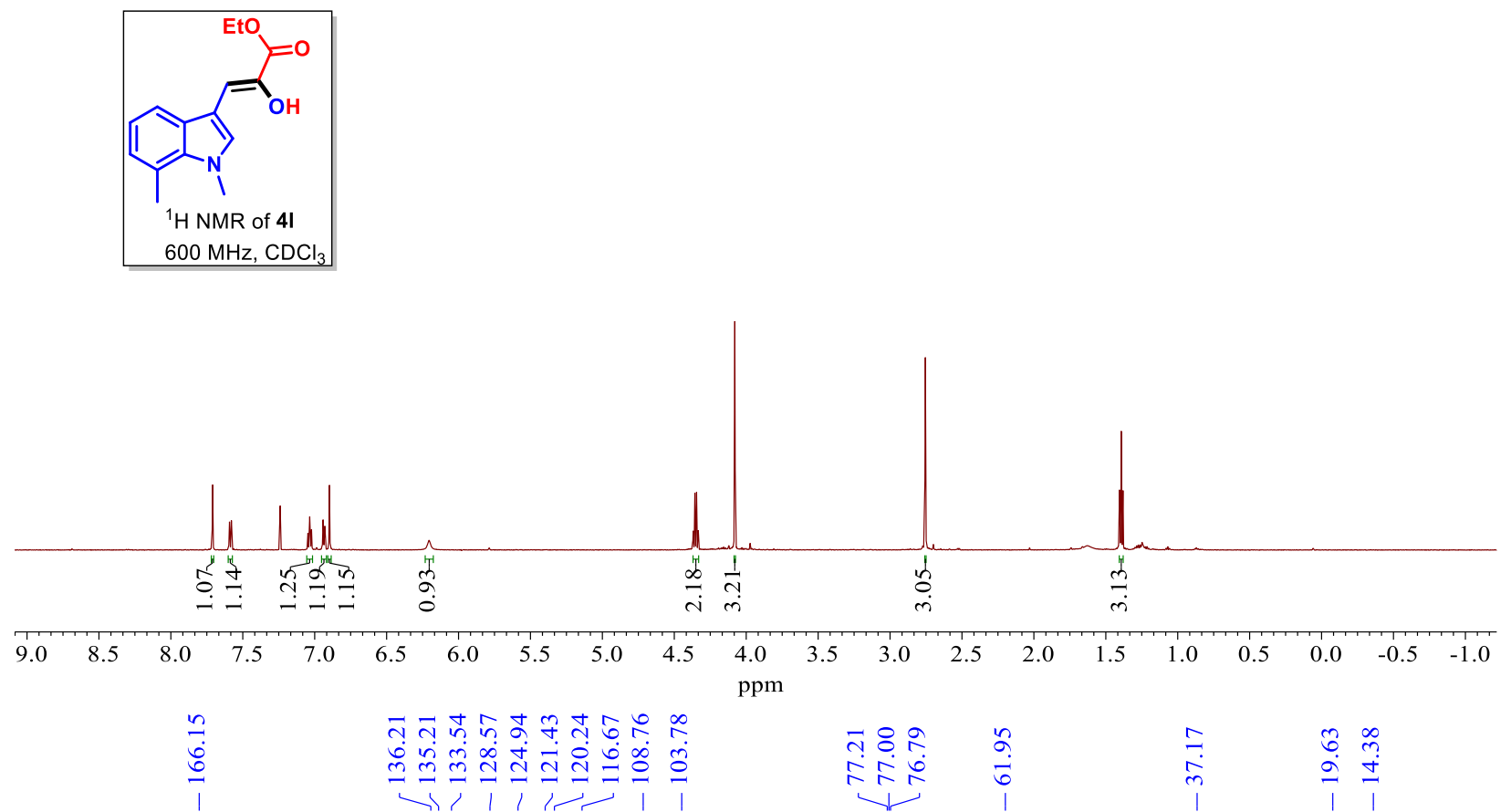

ppm
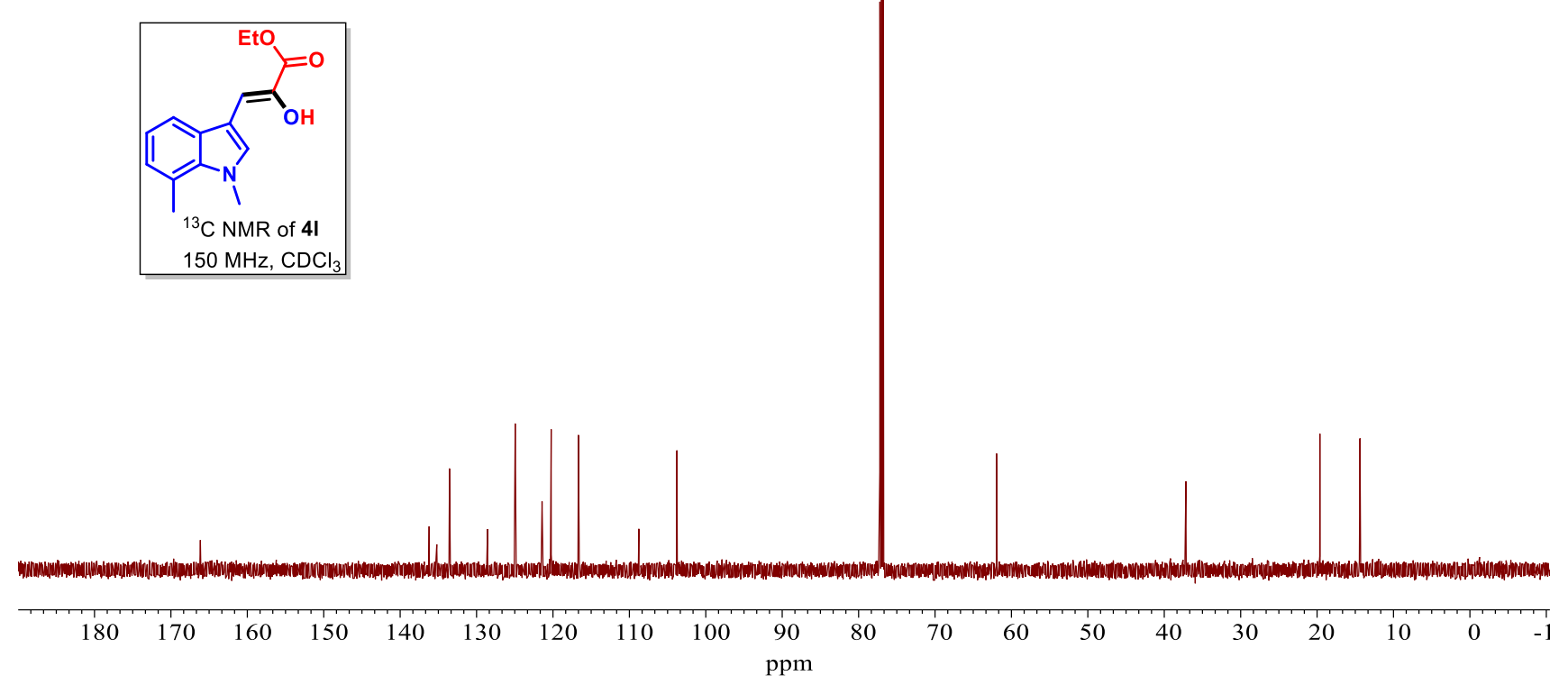

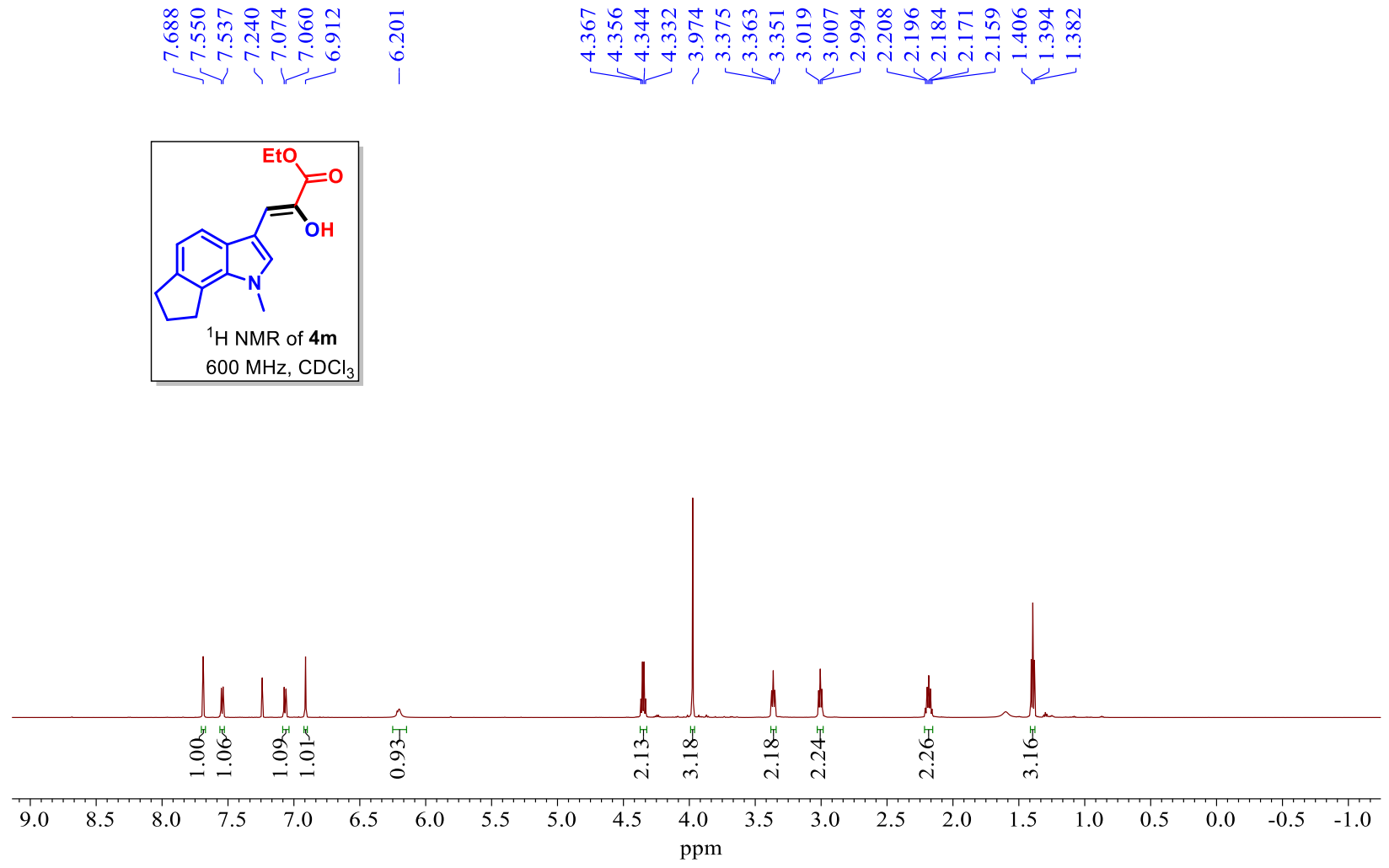

\begin{tabular}{|c|c|c|c|c|c|}
\hline $\begin{array}{l}0 \\
\infty \\
\sigma \\
i \\
0\end{array}$ & 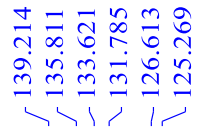 & 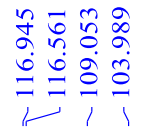 & 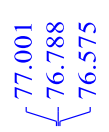 & $\frac{7}{i}$ & 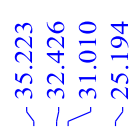 \\
\hline
\end{tabular}
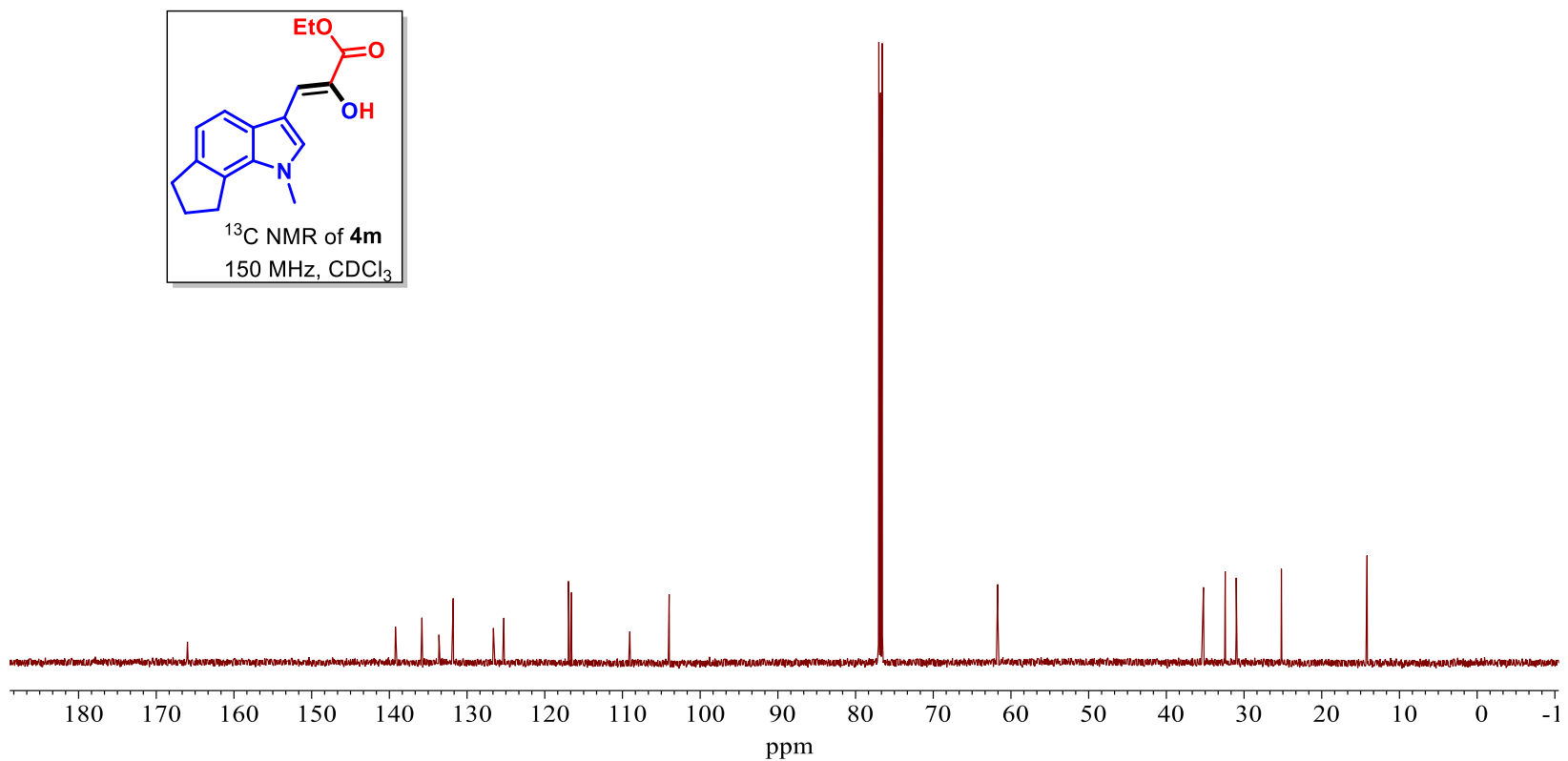

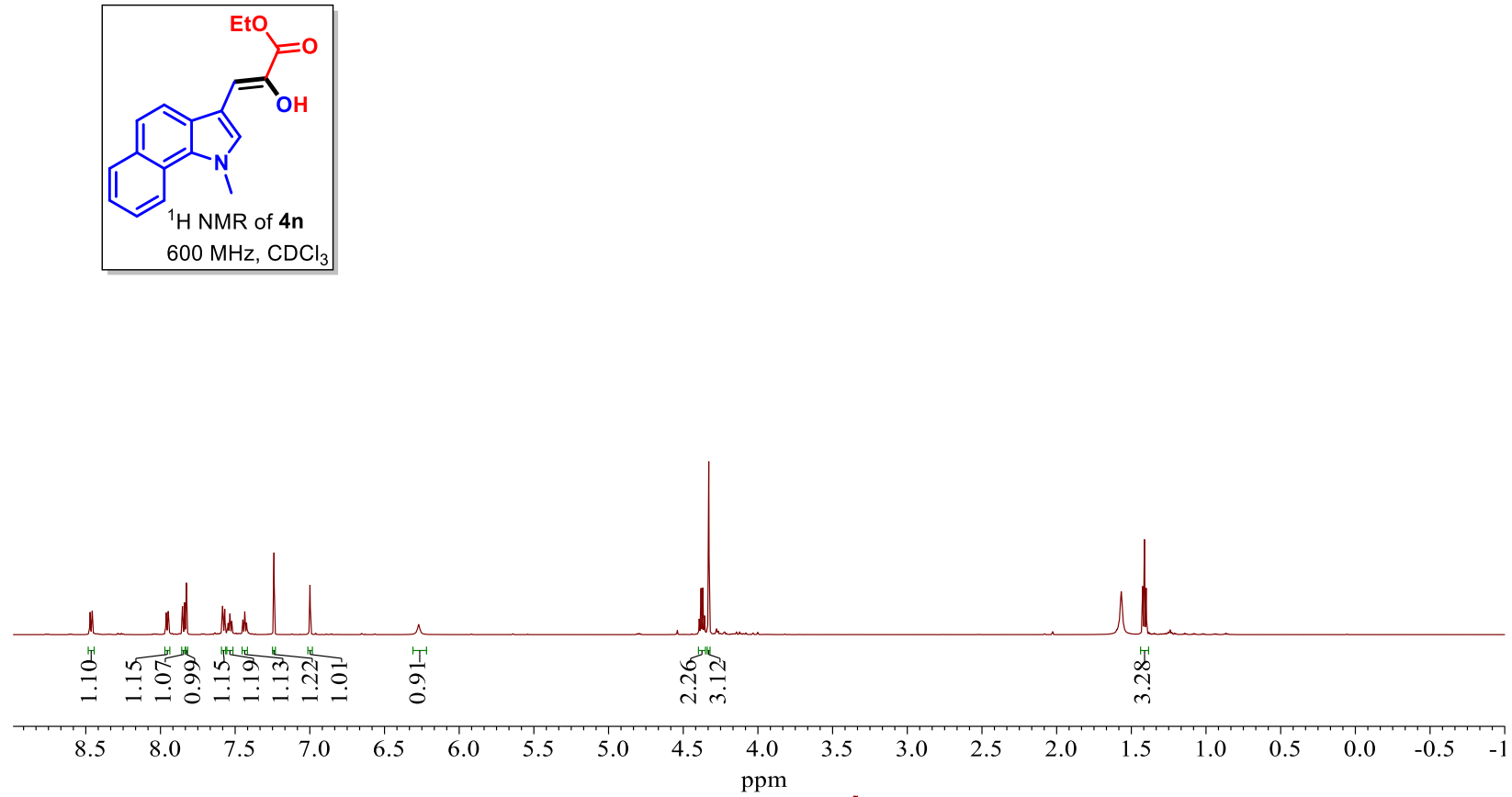

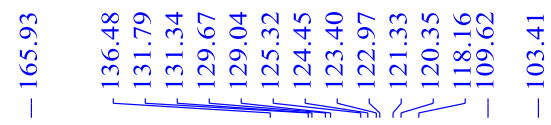

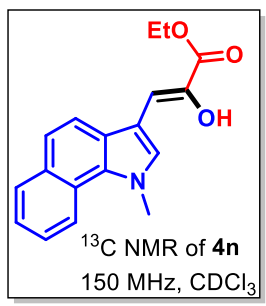

pm

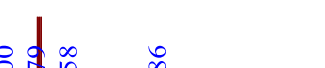

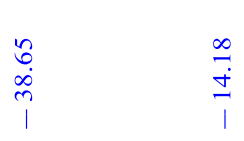

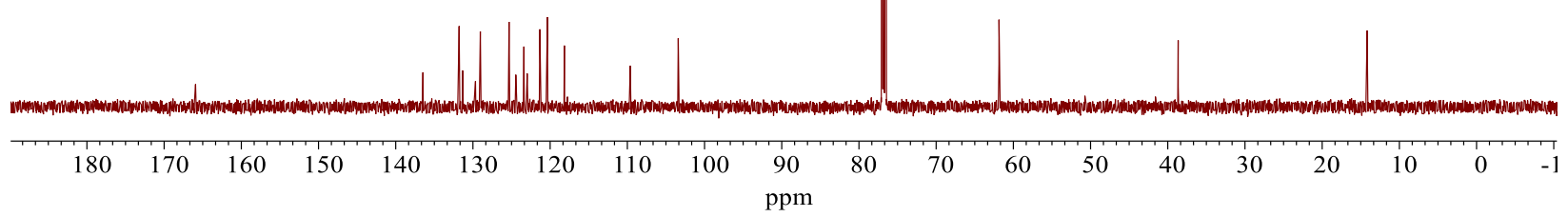




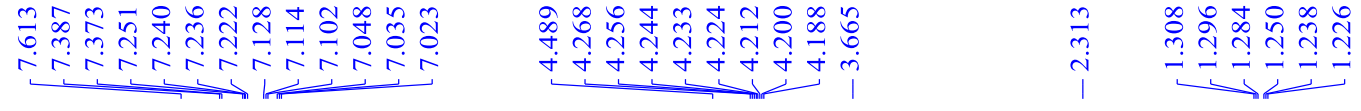

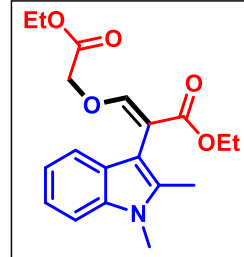

${ }^{1} \mathrm{H}$ NMR of $\mathbf{6 a}$

$600 \mathrm{MHz} \mathrm{CDCl}_{3}$

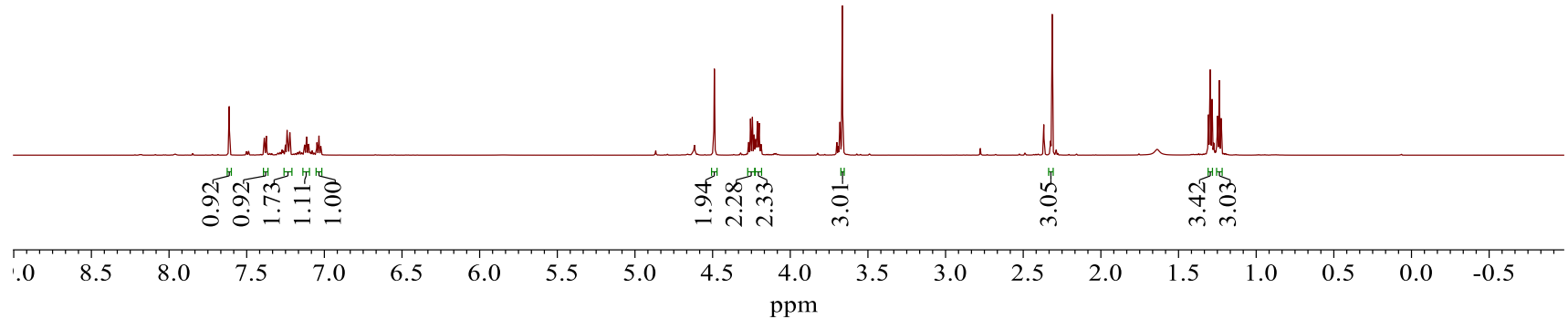

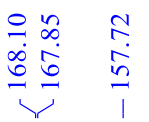

Ұ

ஸ

।

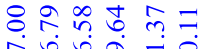

$\therefore \leqslant 06$

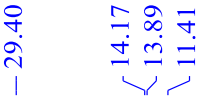
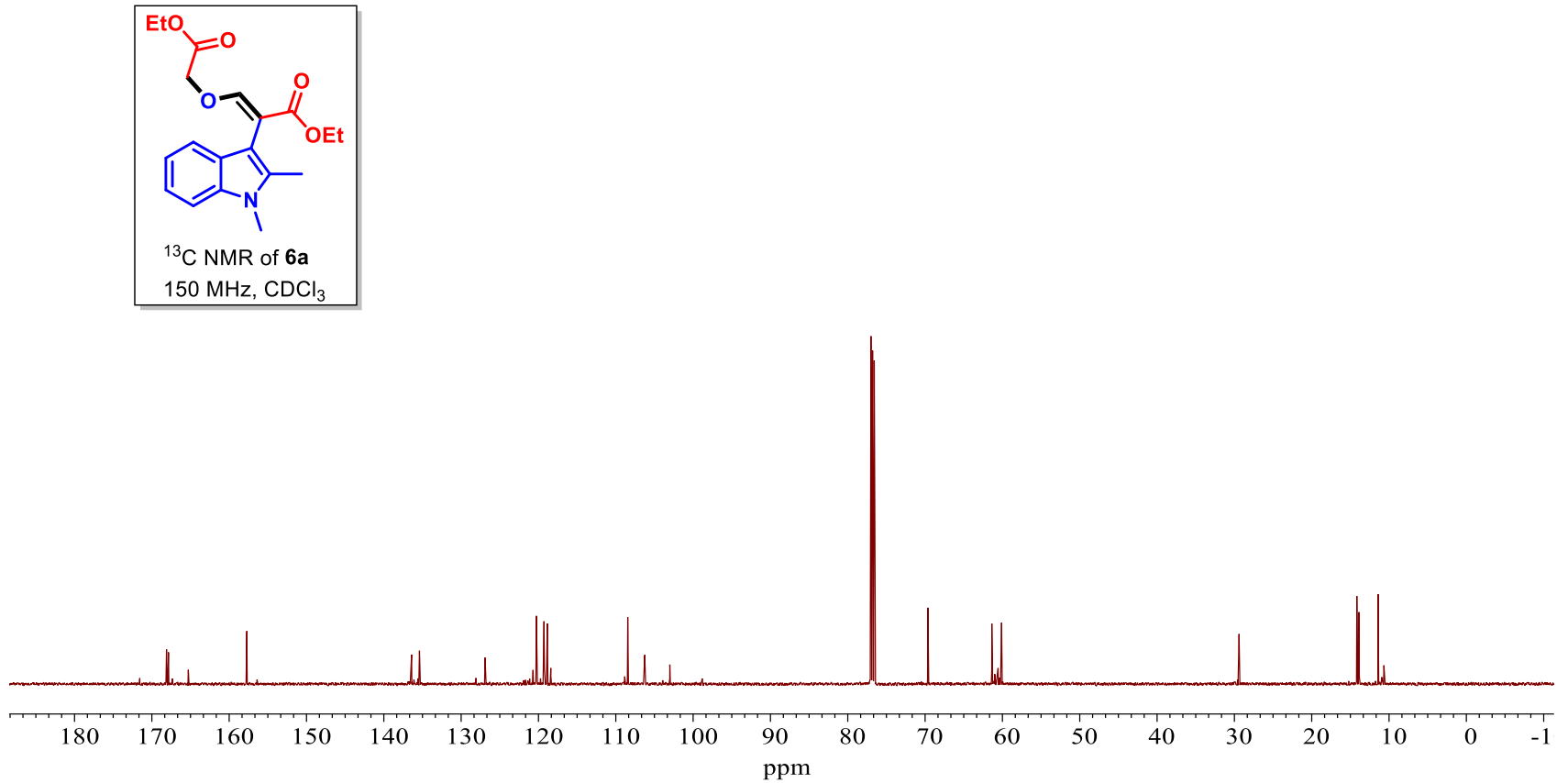


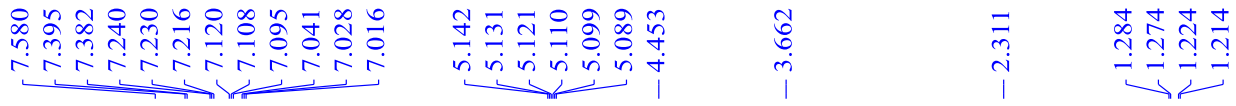
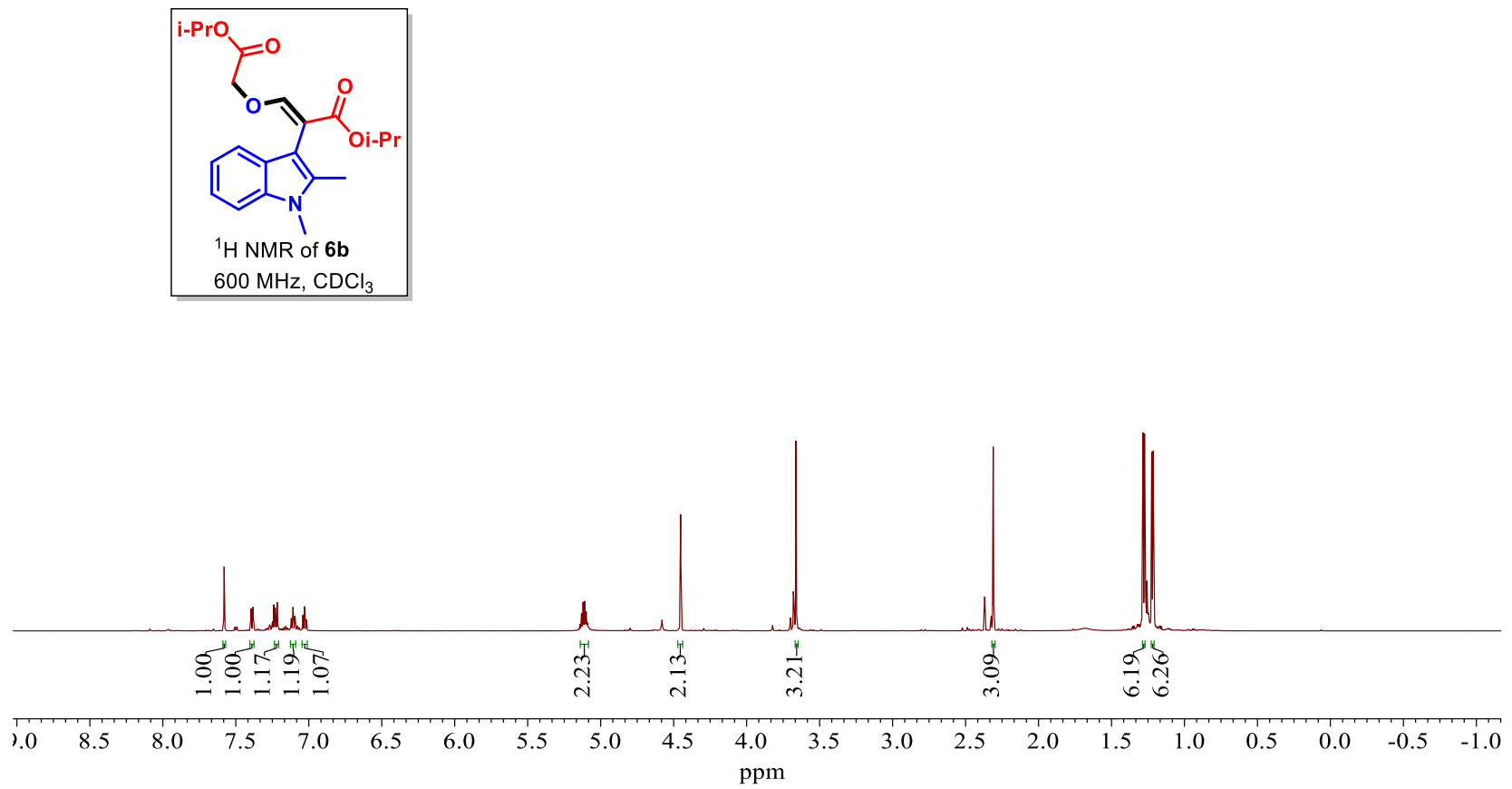

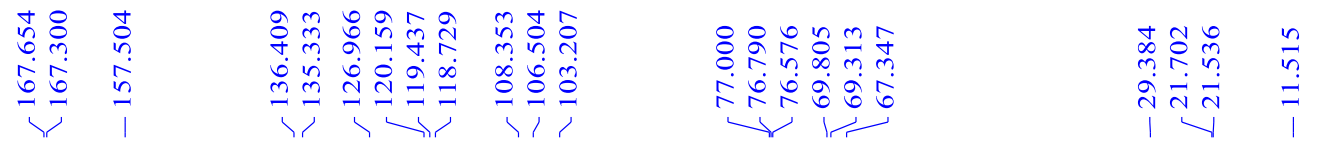

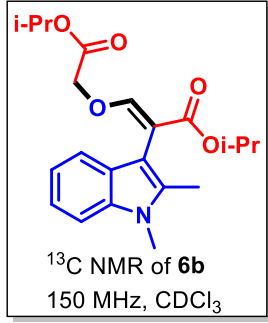



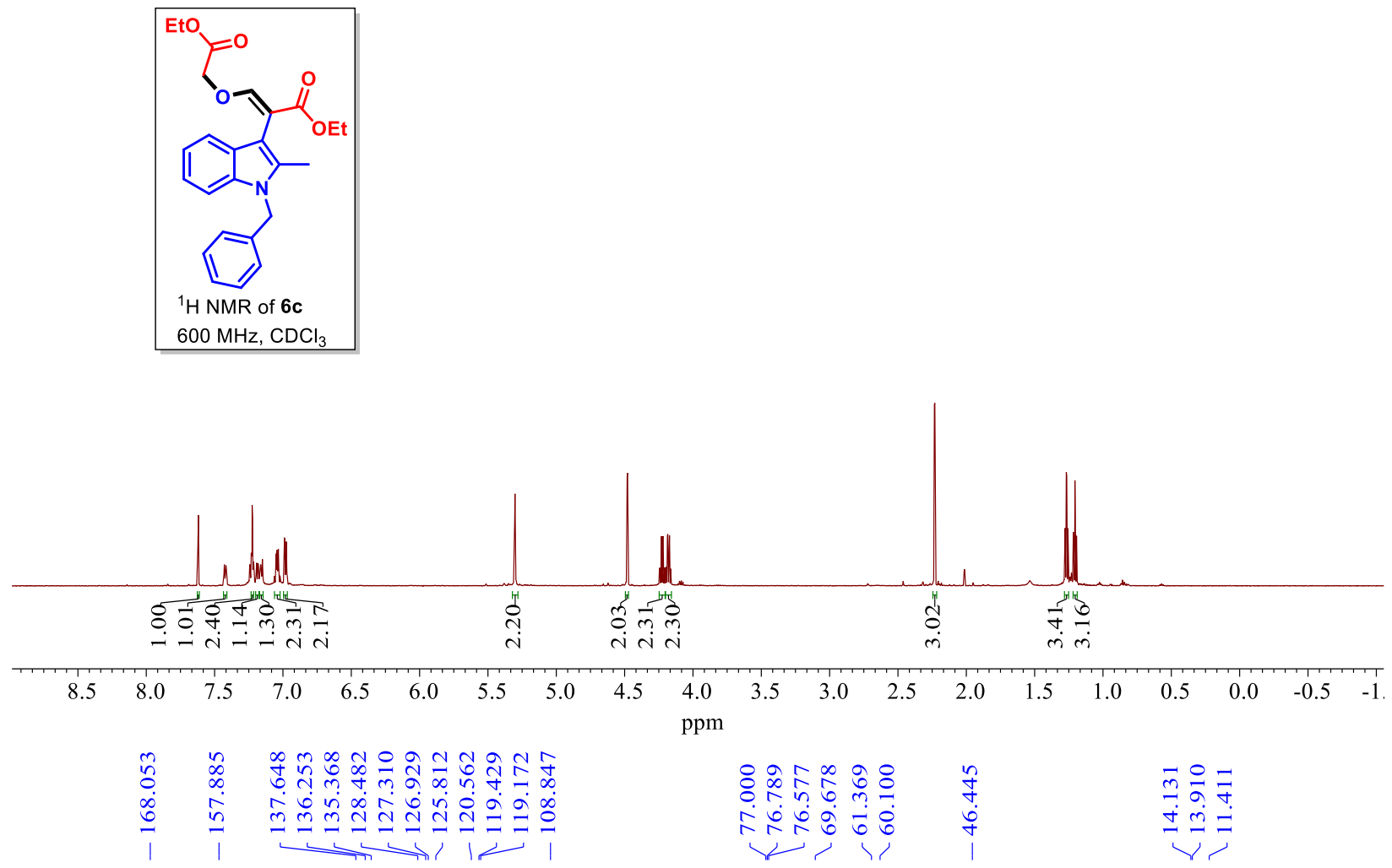

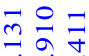

$\stackrel{\sim}{=}$
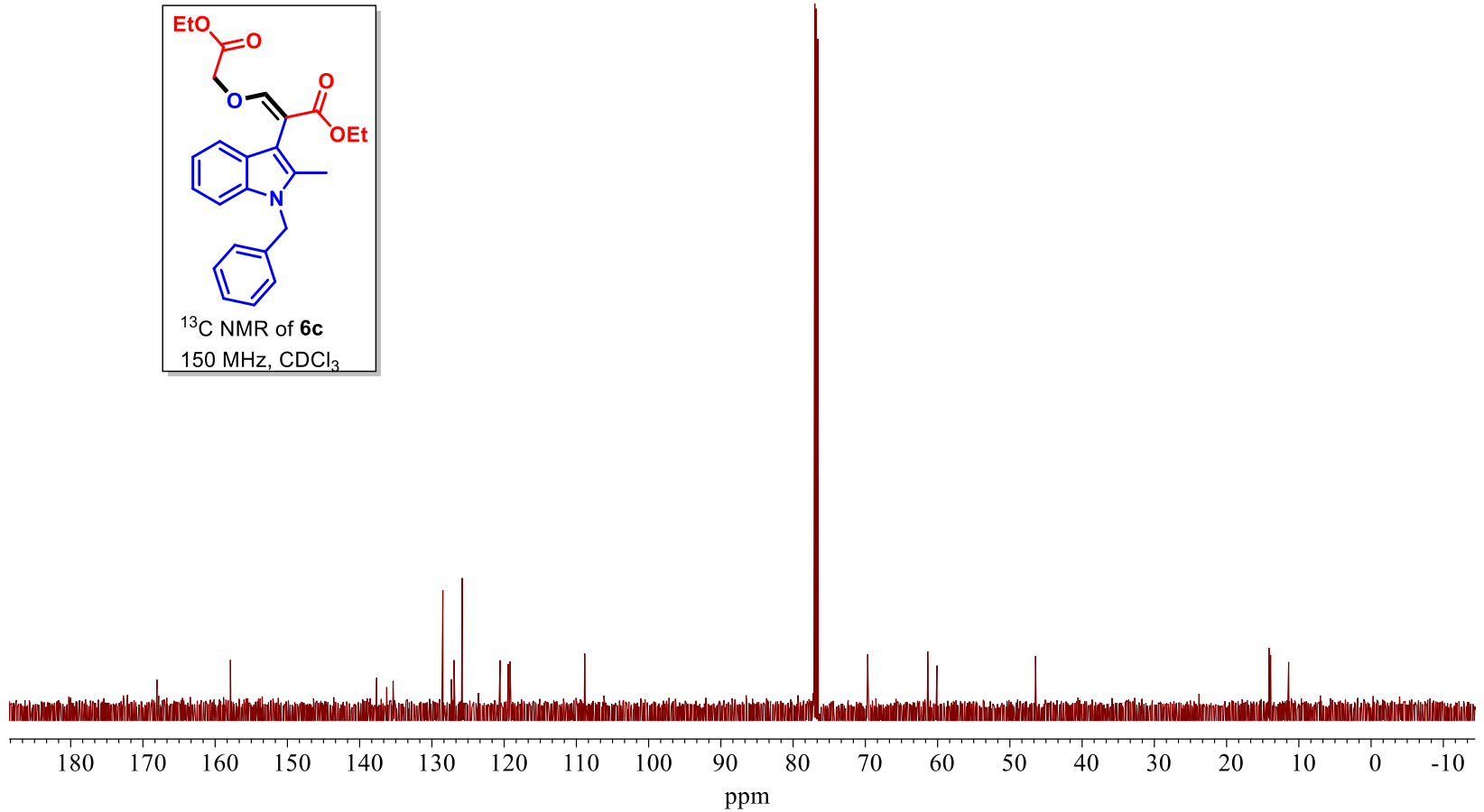


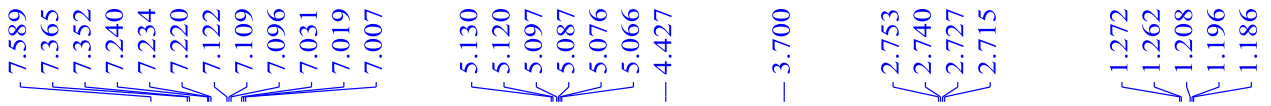
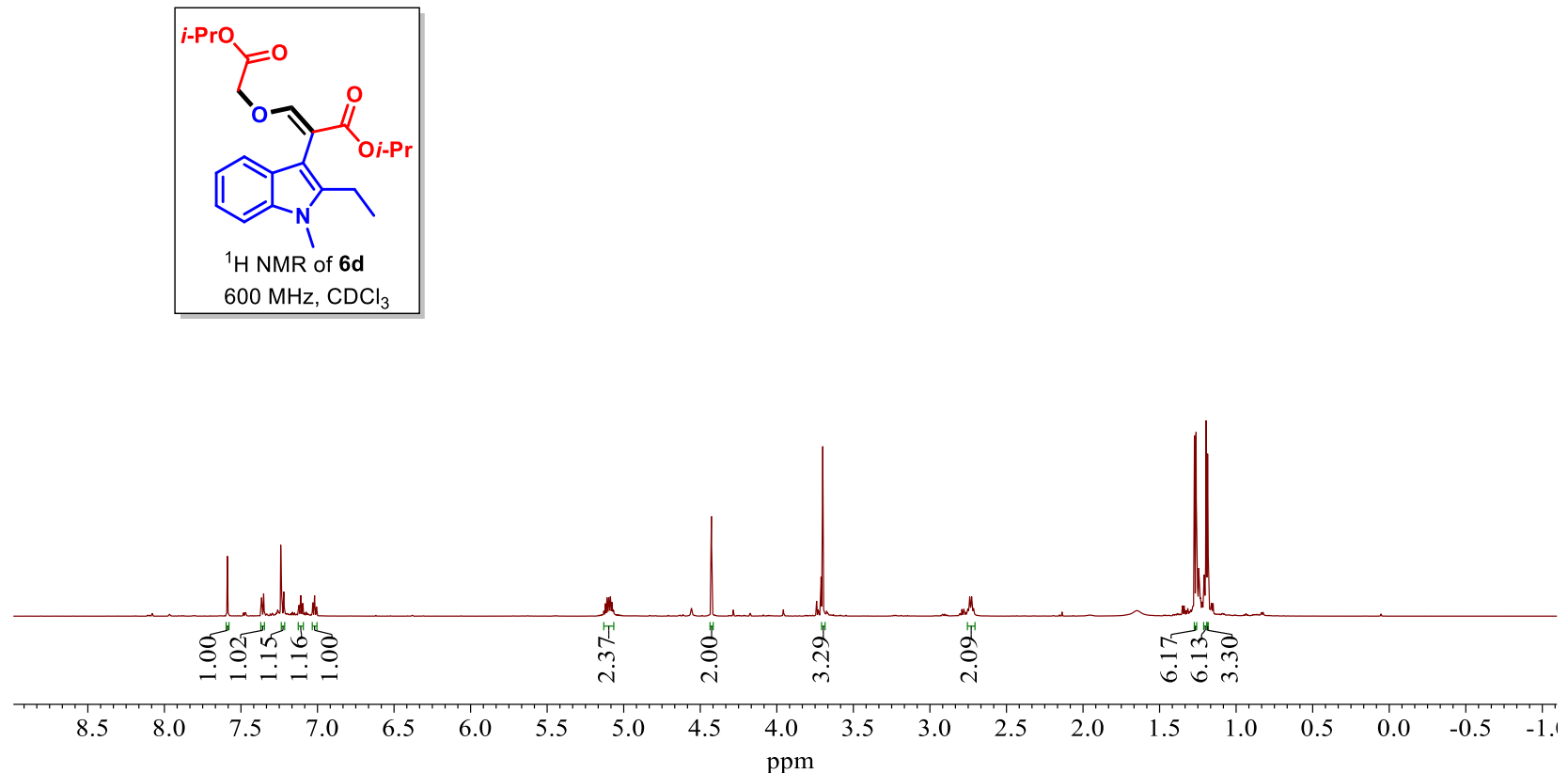

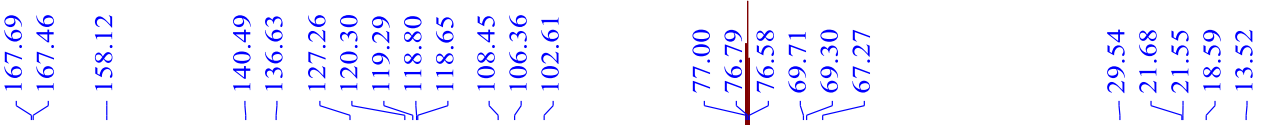
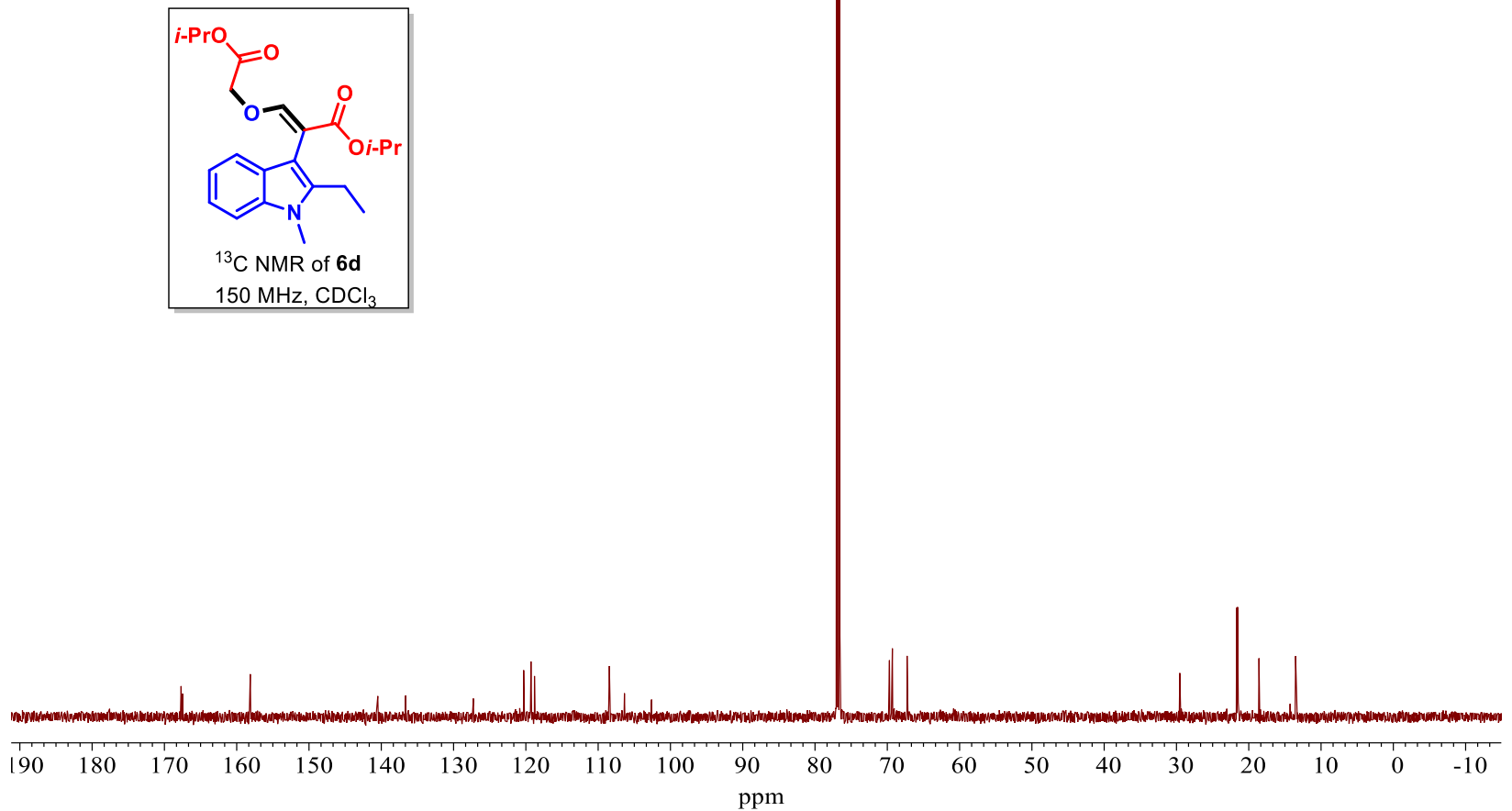


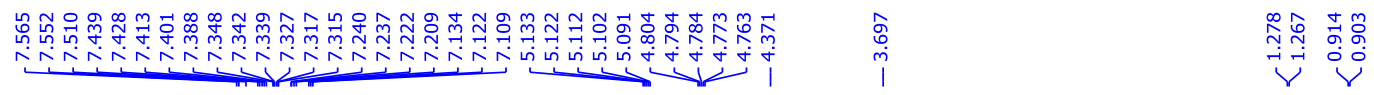
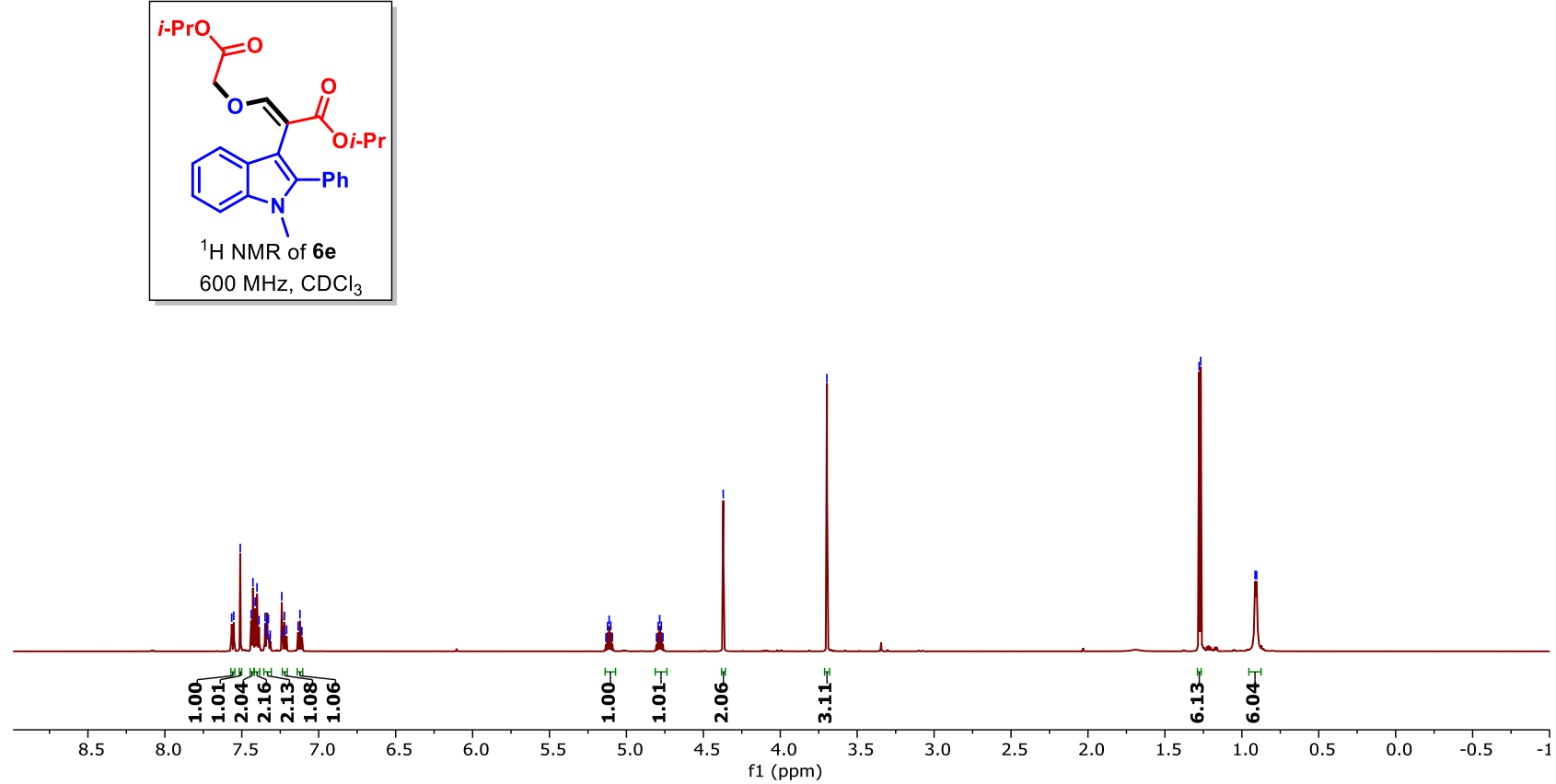

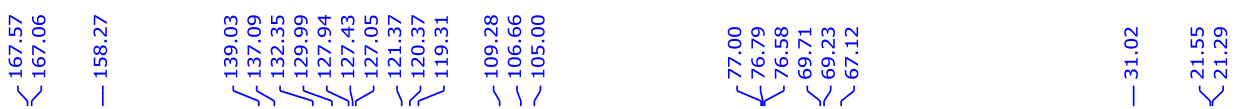
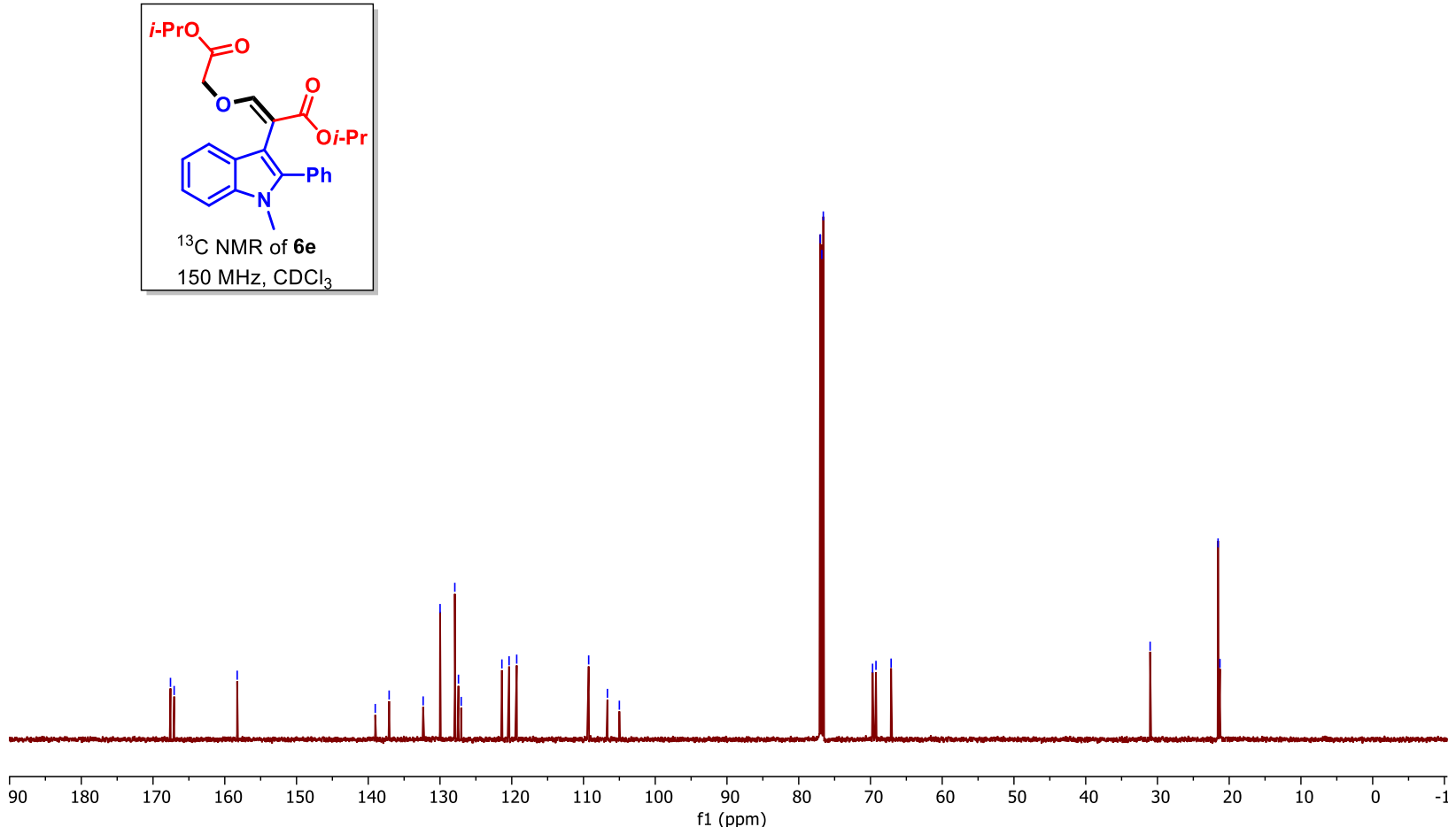

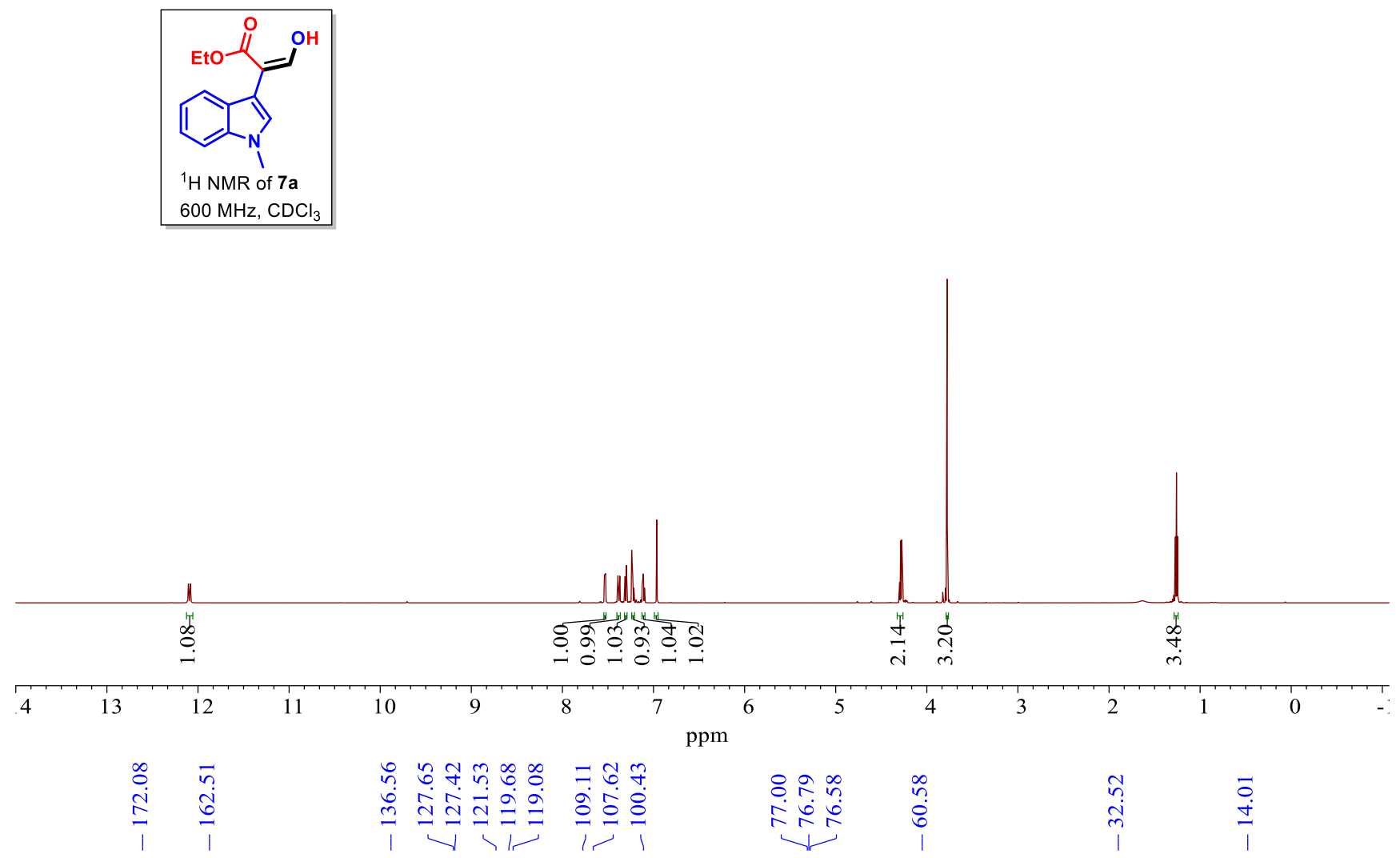

$$
\text { ppm }
$$

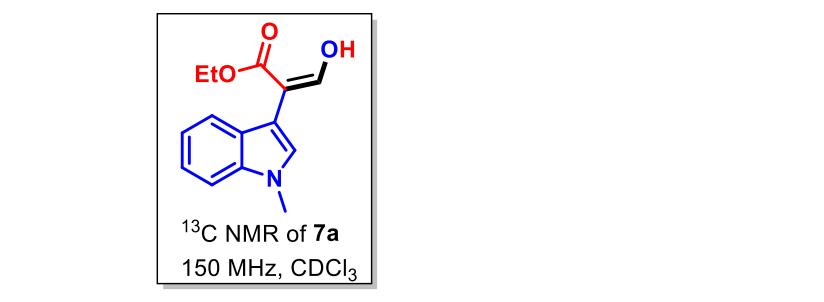

\begin{tabular}{lll}
1 & \multicolumn{1}{l}{1} \\
0 & 1
\end{tabular}

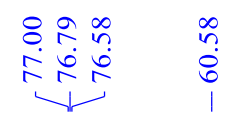

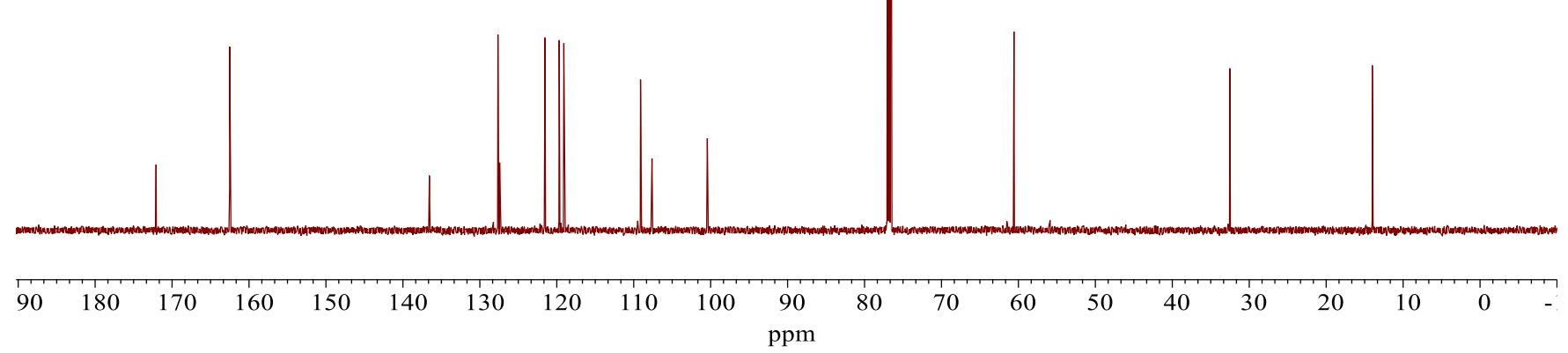




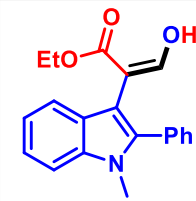

${ }^{1} \mathrm{H}$ NMR of $7 b$ $600 \mathrm{MHz}, \mathrm{CDCl}_{3}$

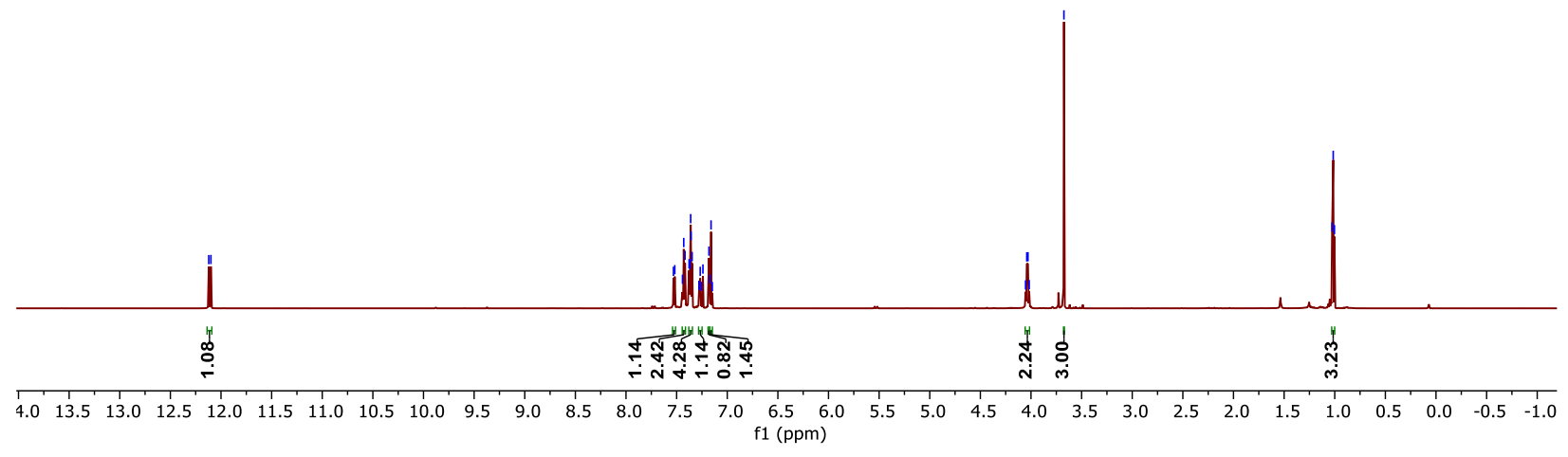

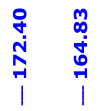

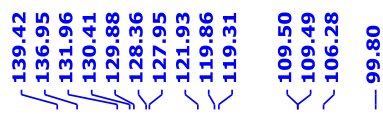

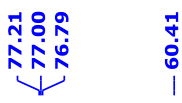

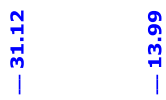

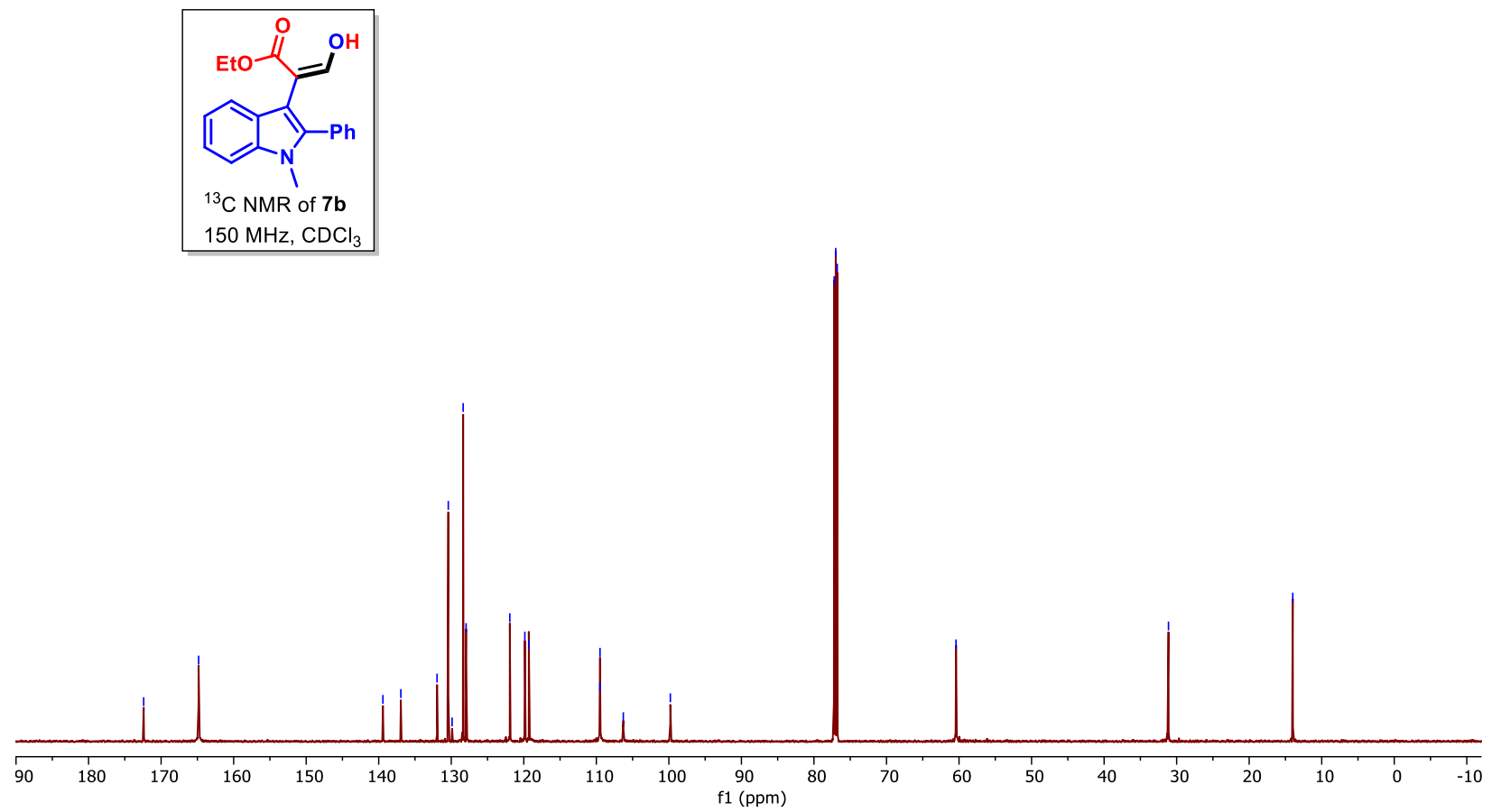



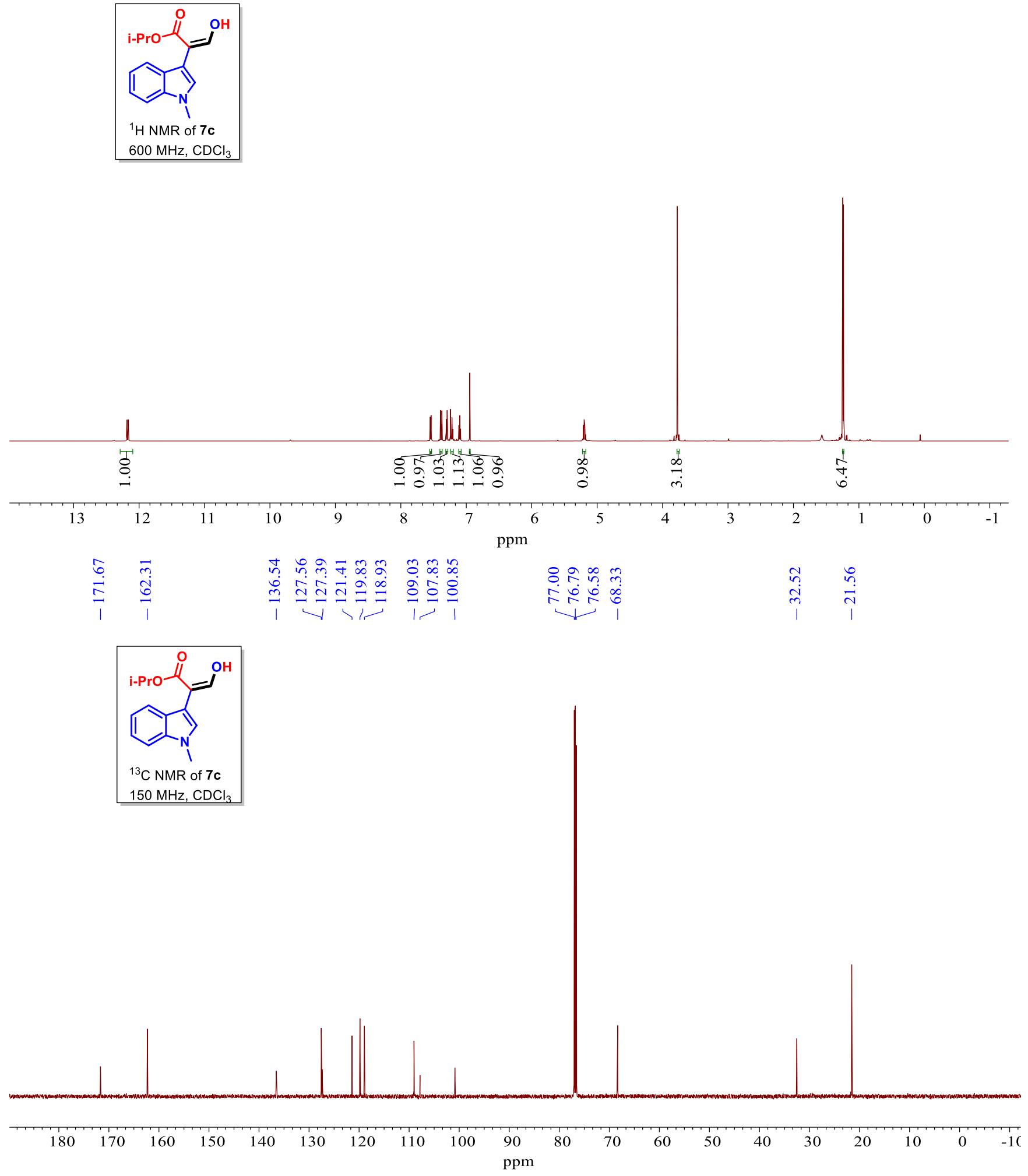


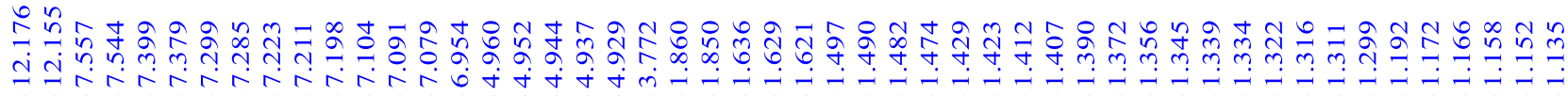
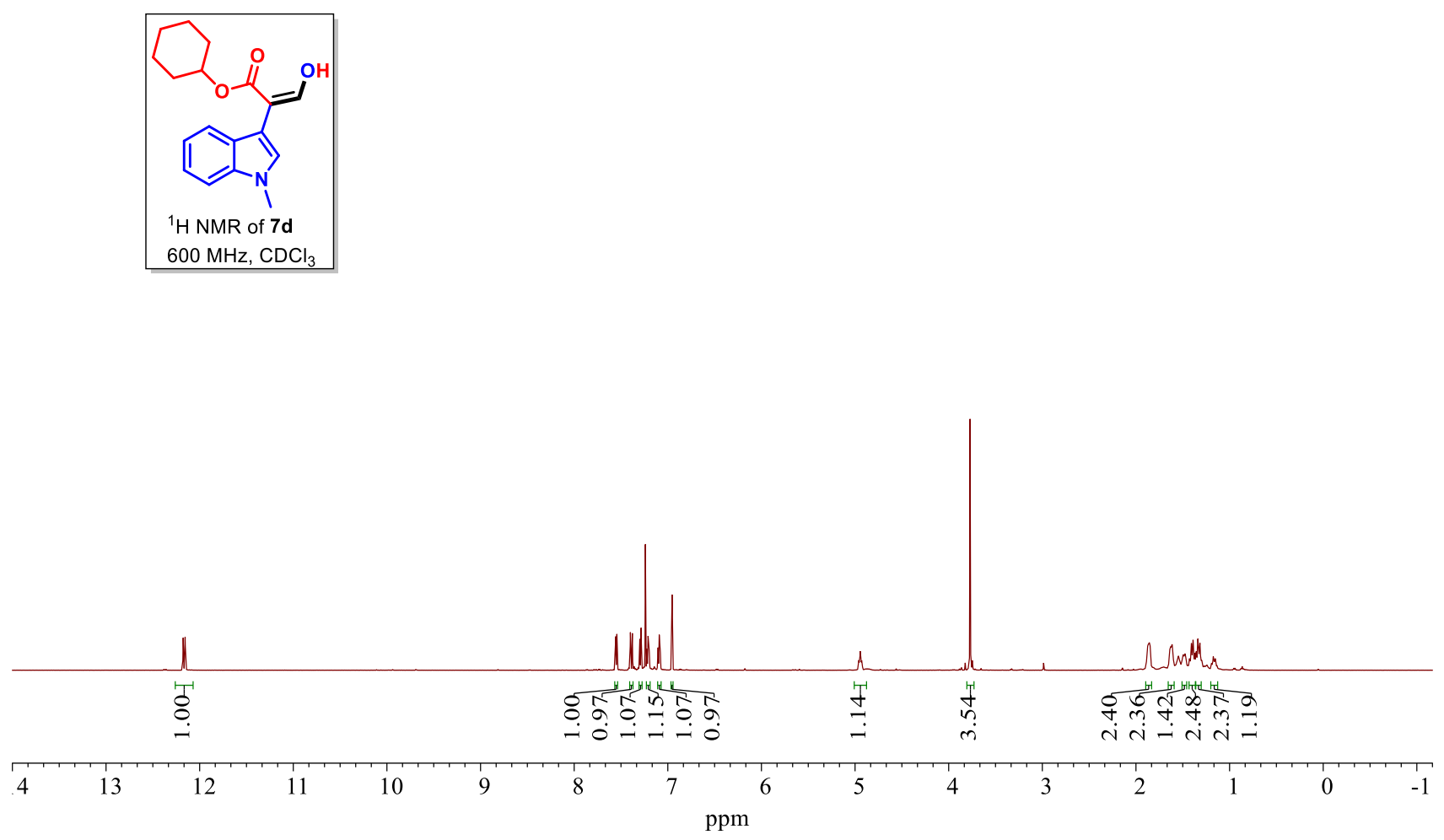

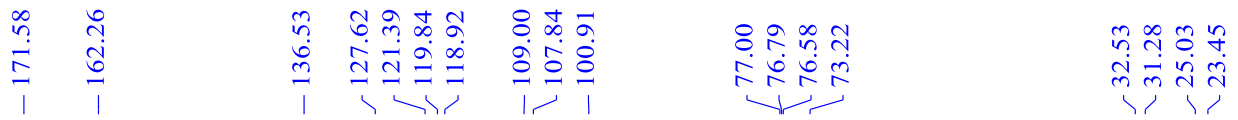
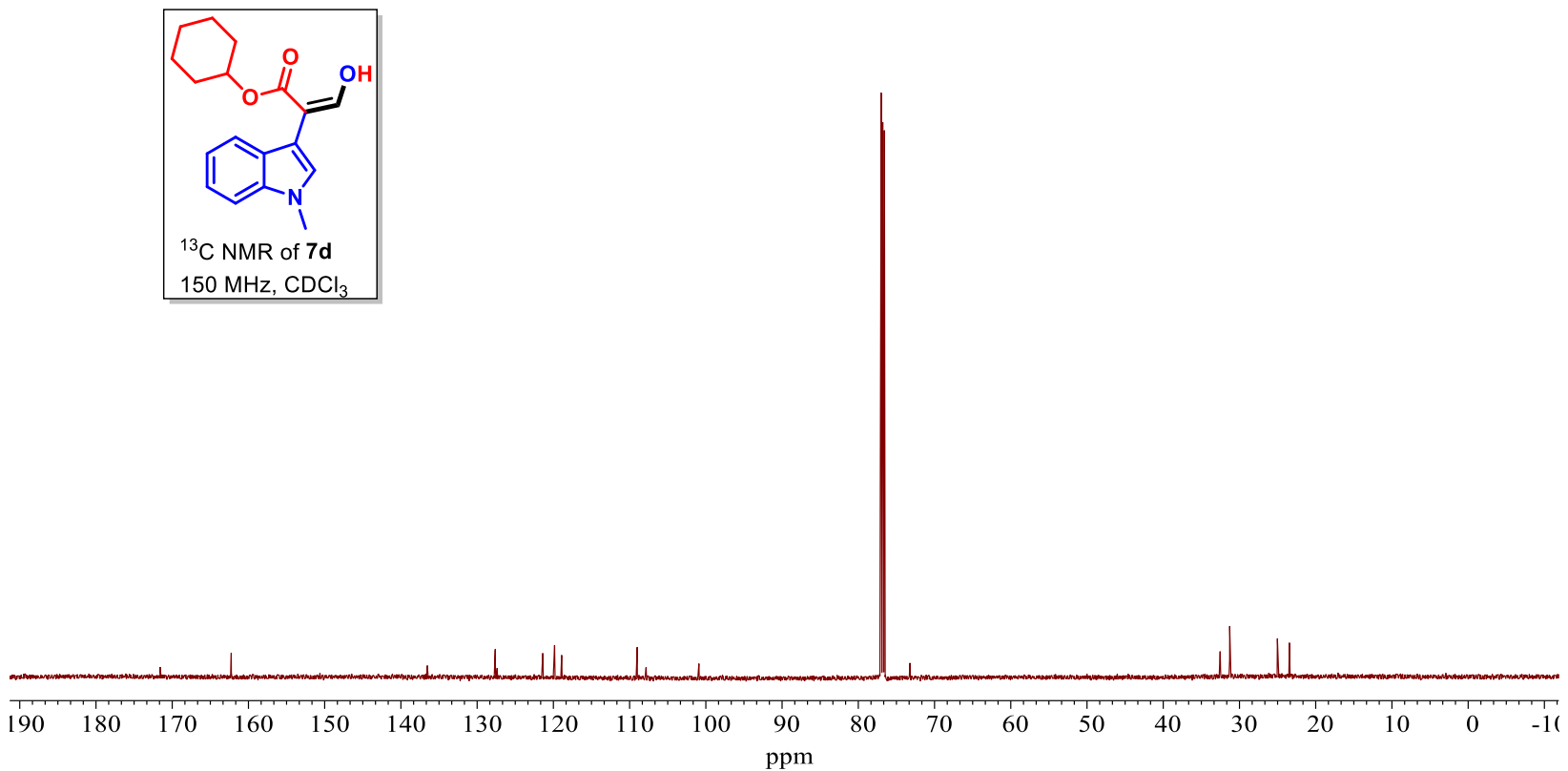

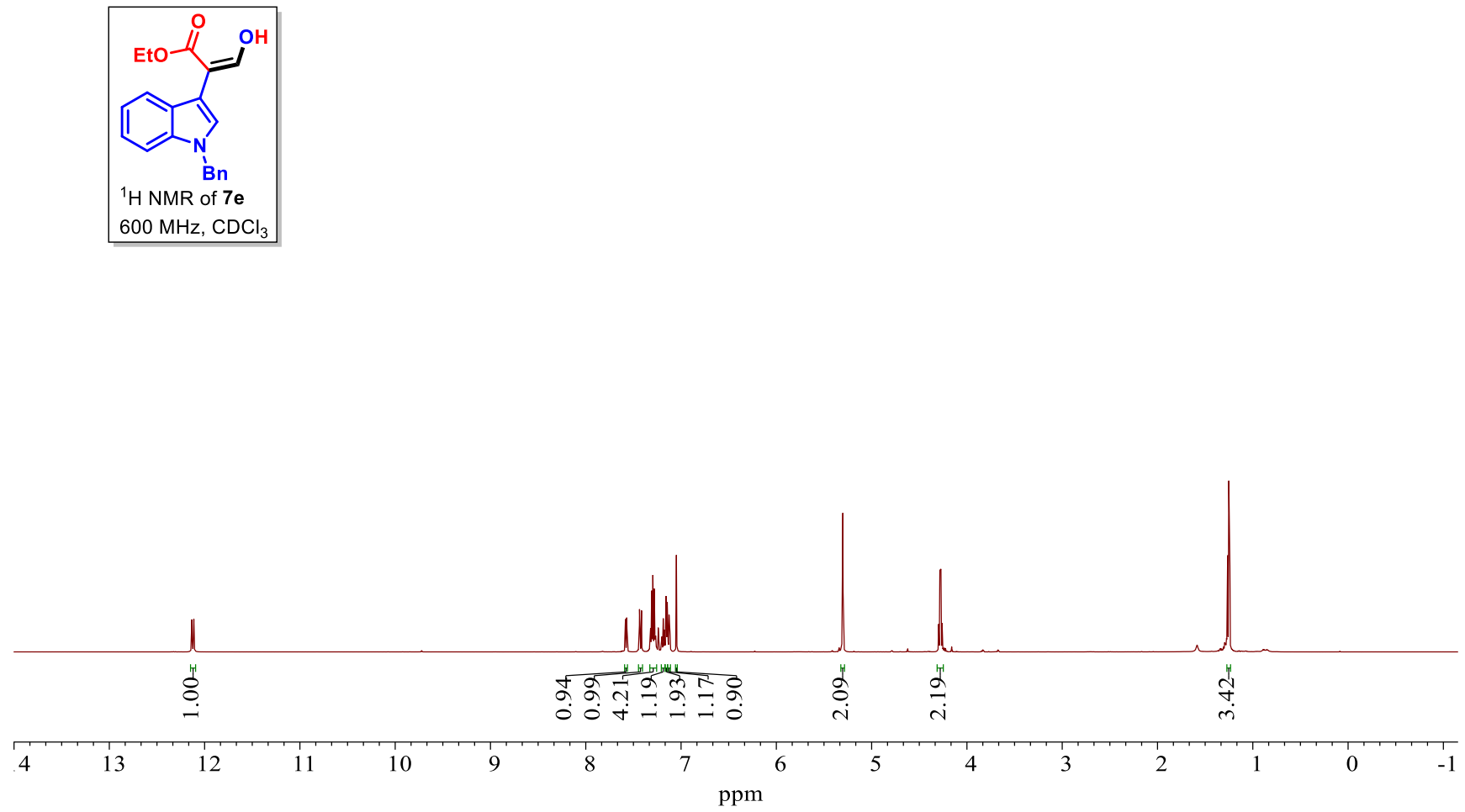

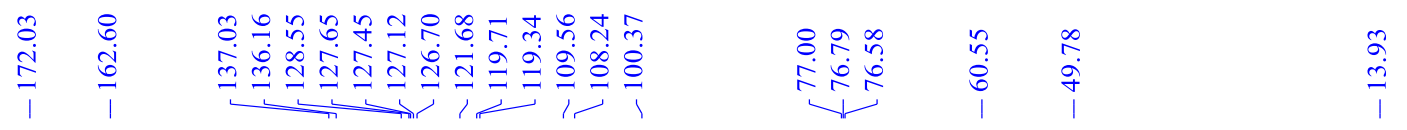

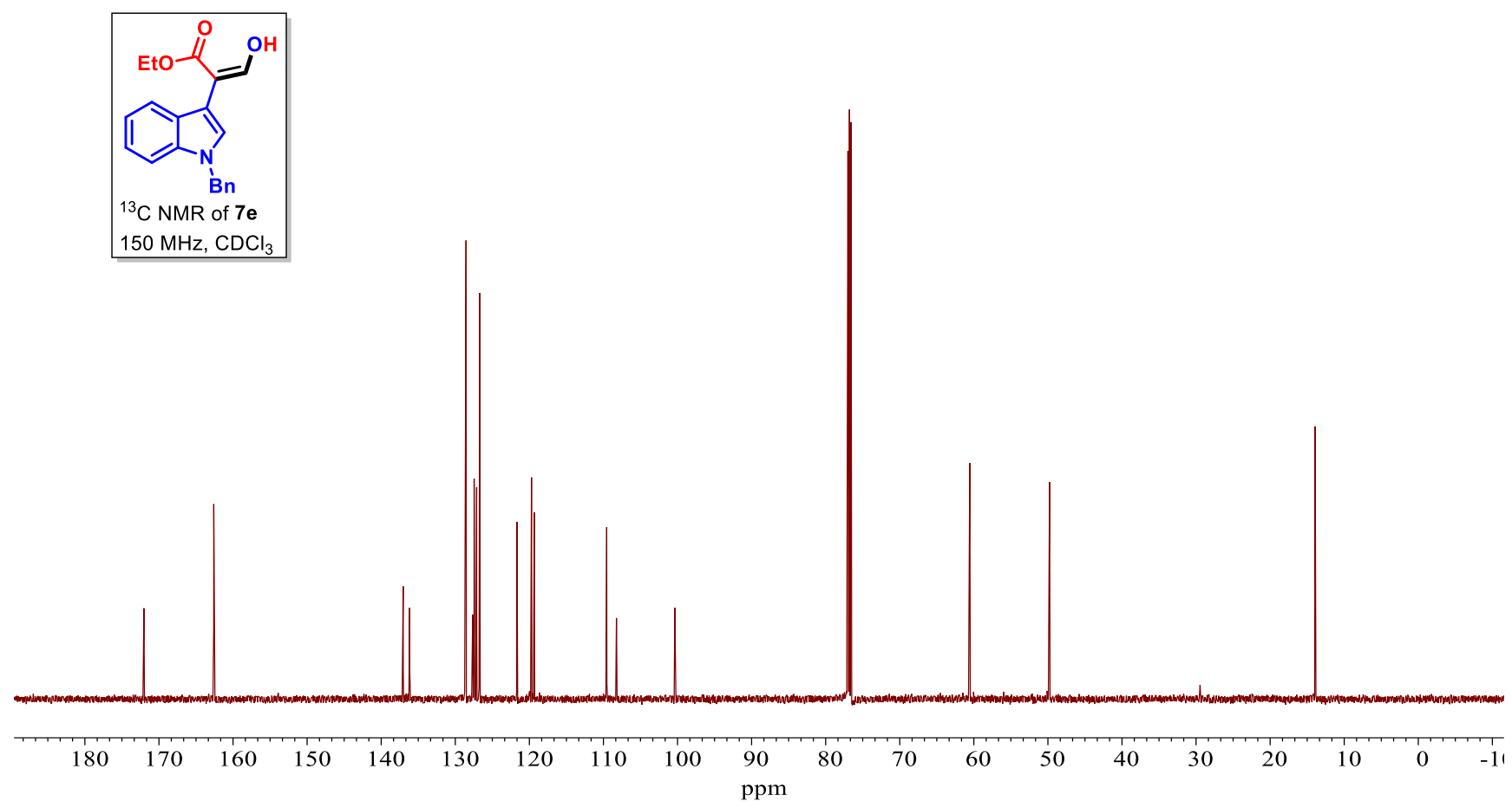




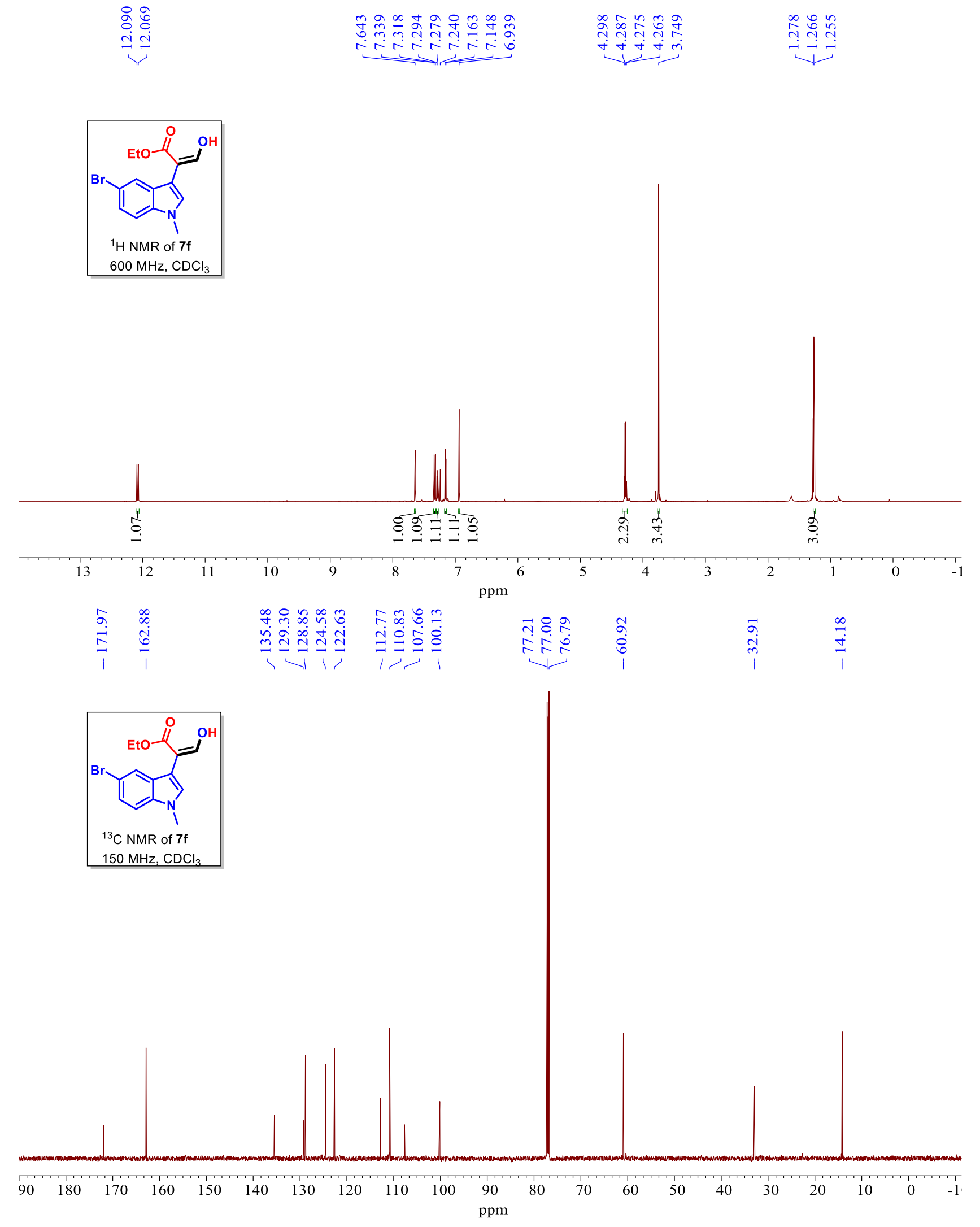




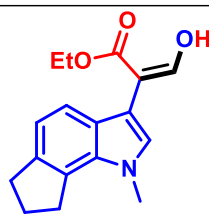

${ }^{1} \mathrm{H}$ NMR of $7 \mathbf{g}$

$600 \mathrm{MHz}, \mathrm{CDCl}_{3}$
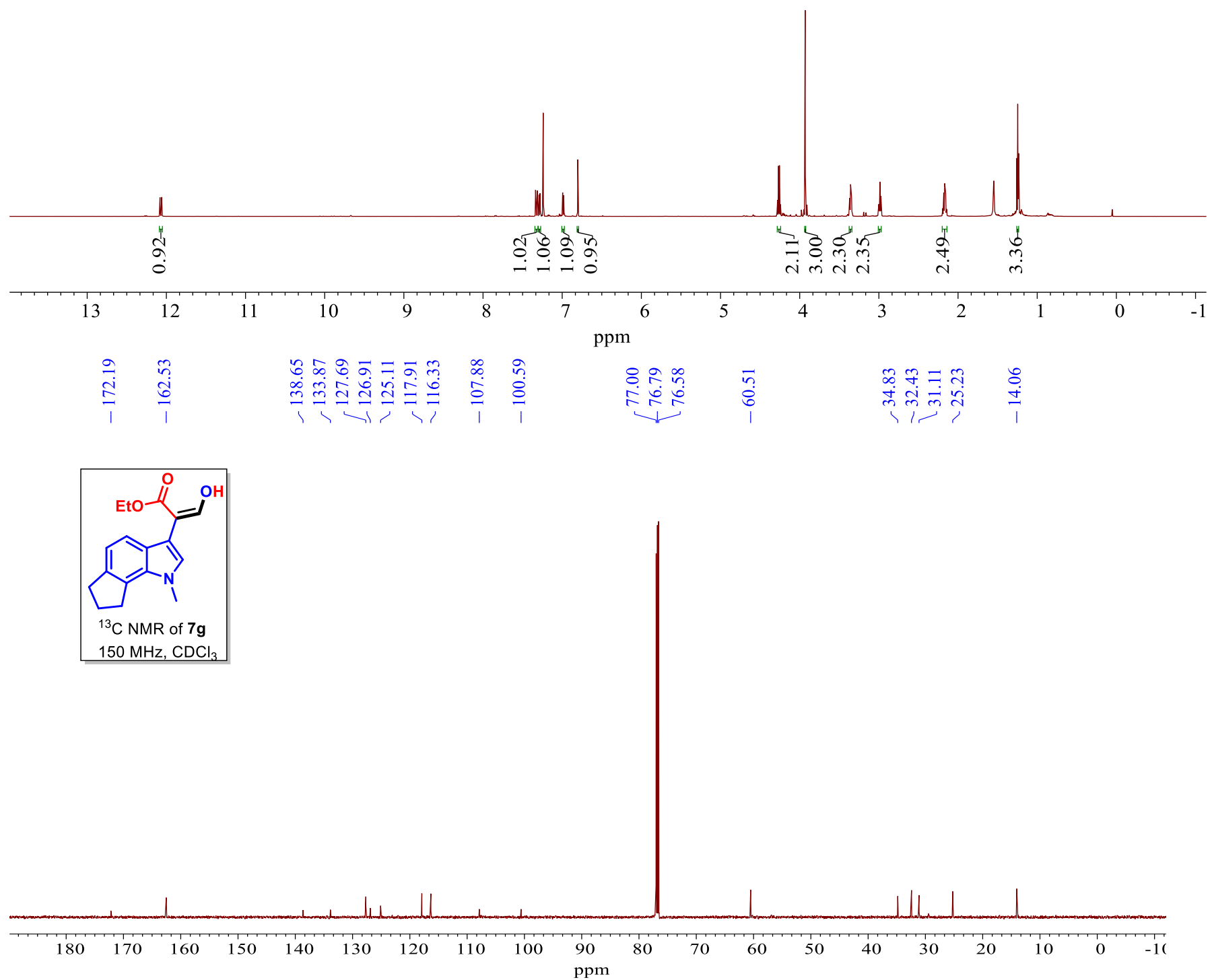

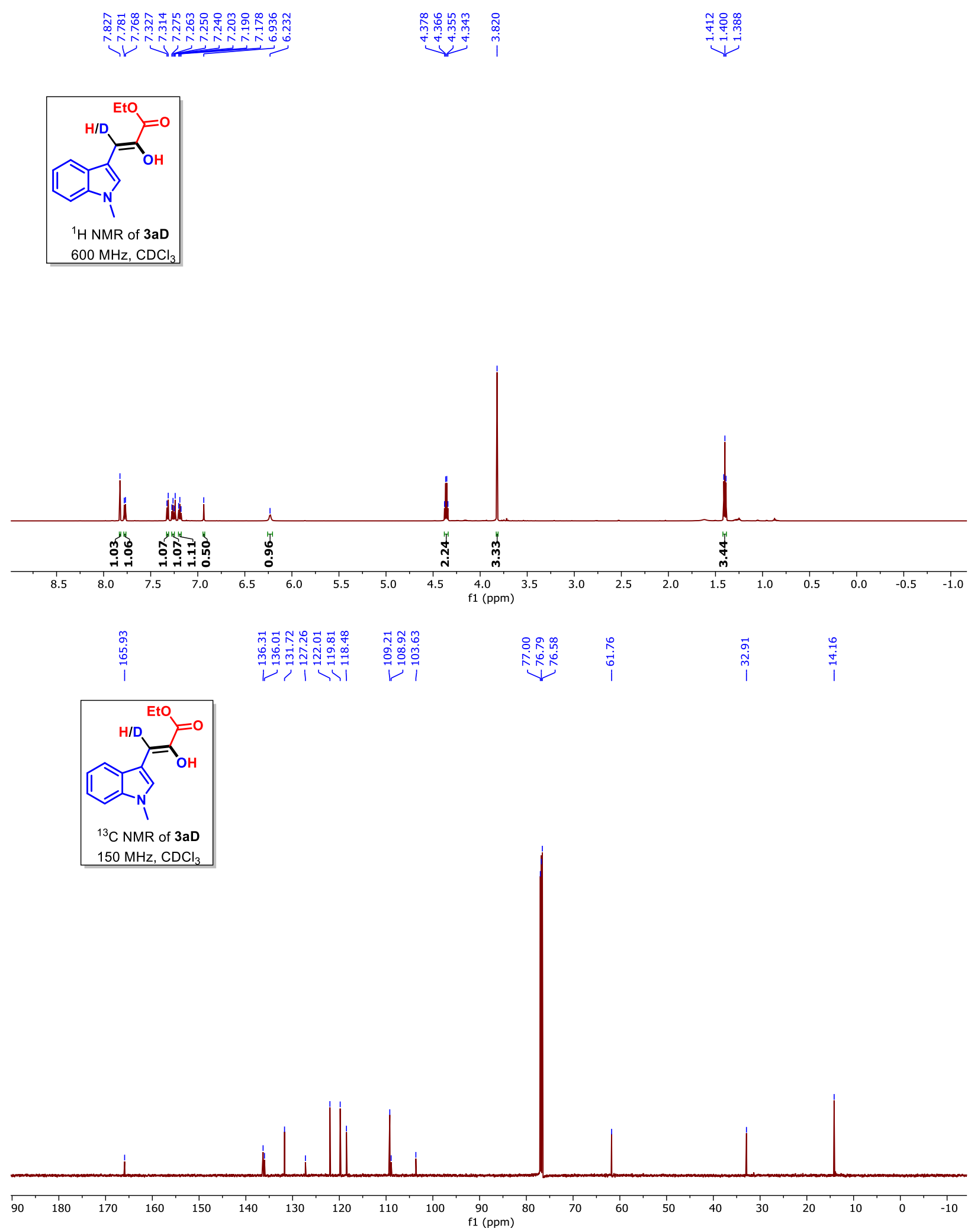


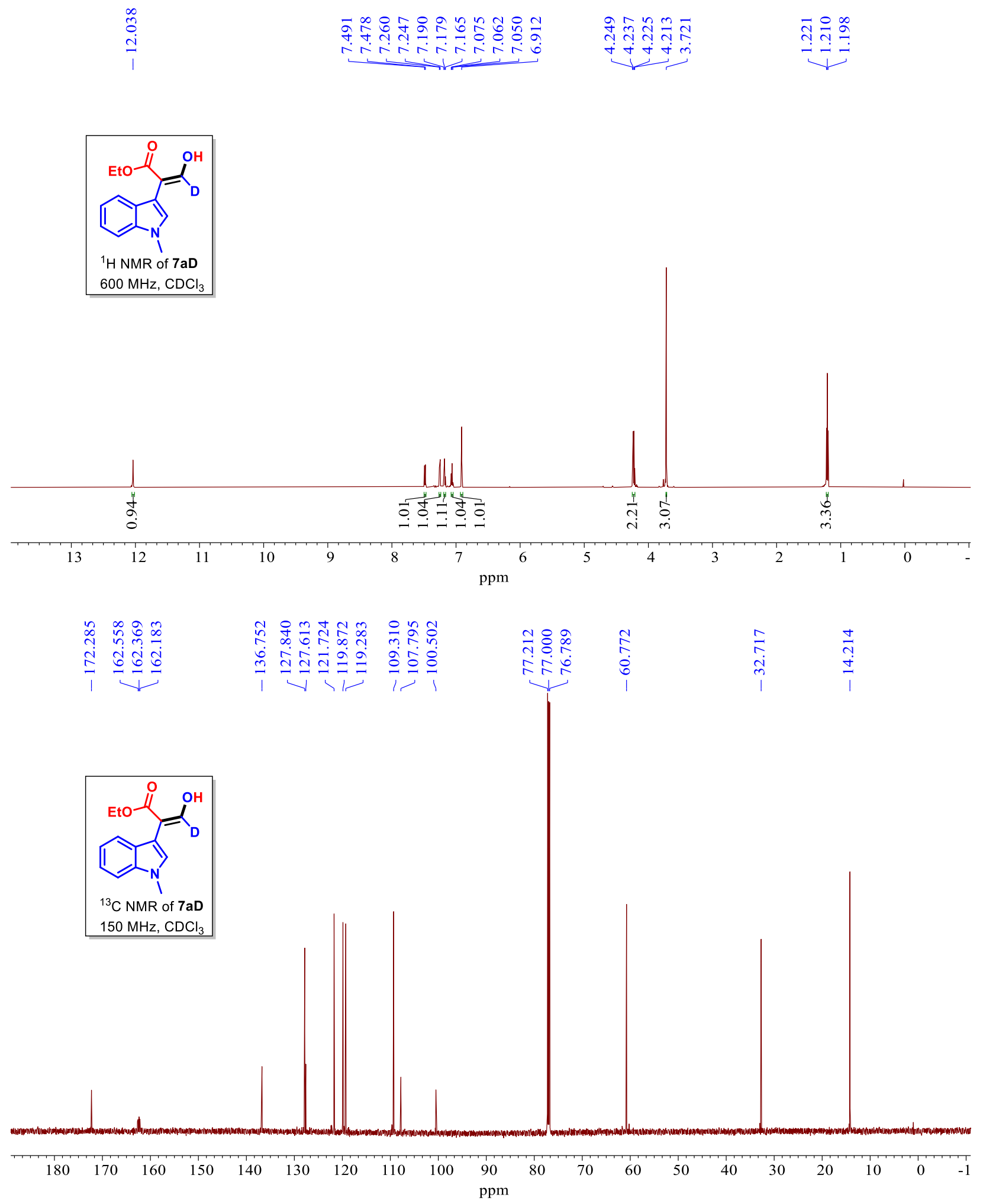



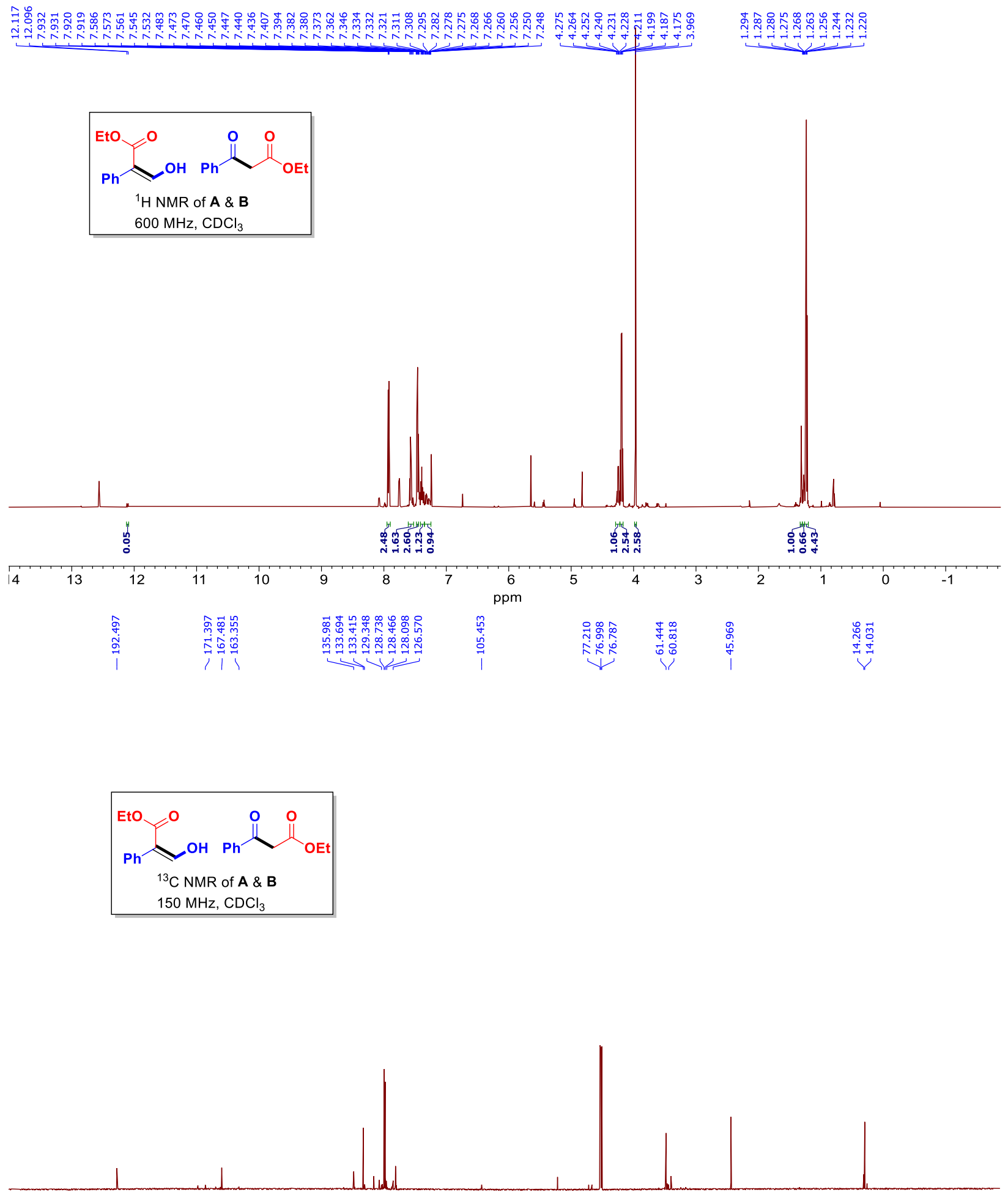

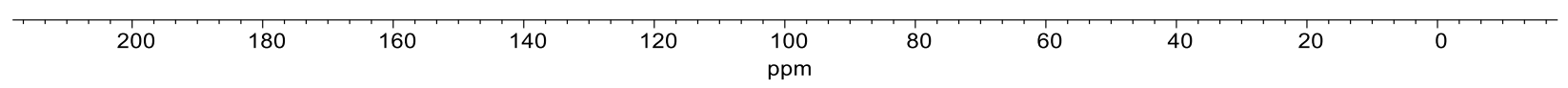




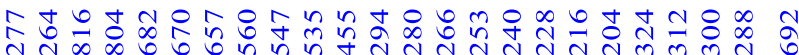

กิ

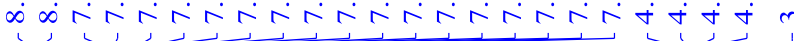

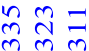

-
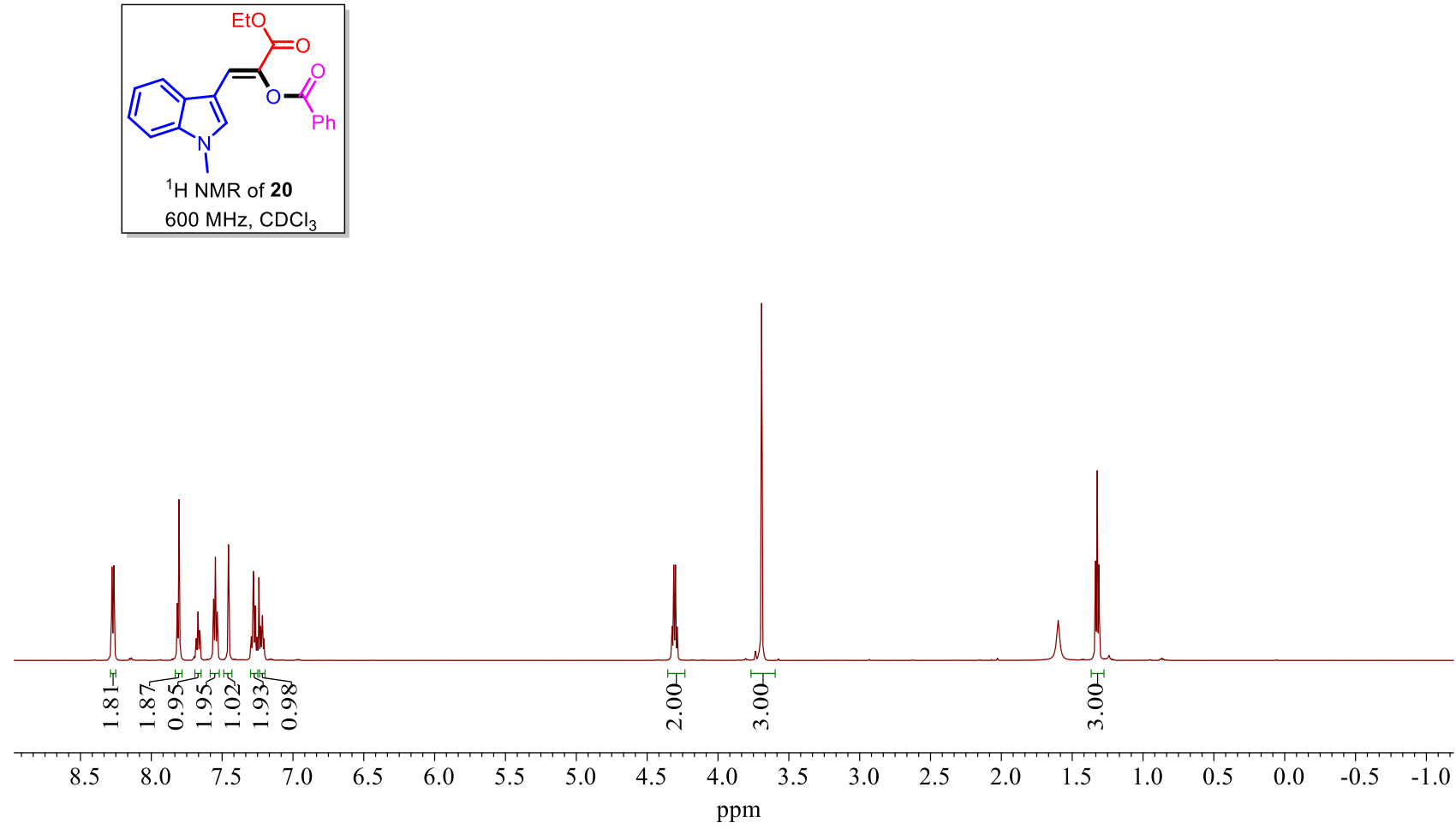

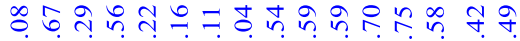

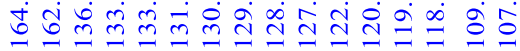

प

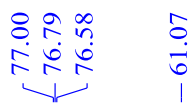

$\stackrel{\substack{\infty \\ m}}{\stackrel{0}{ \pm}}$
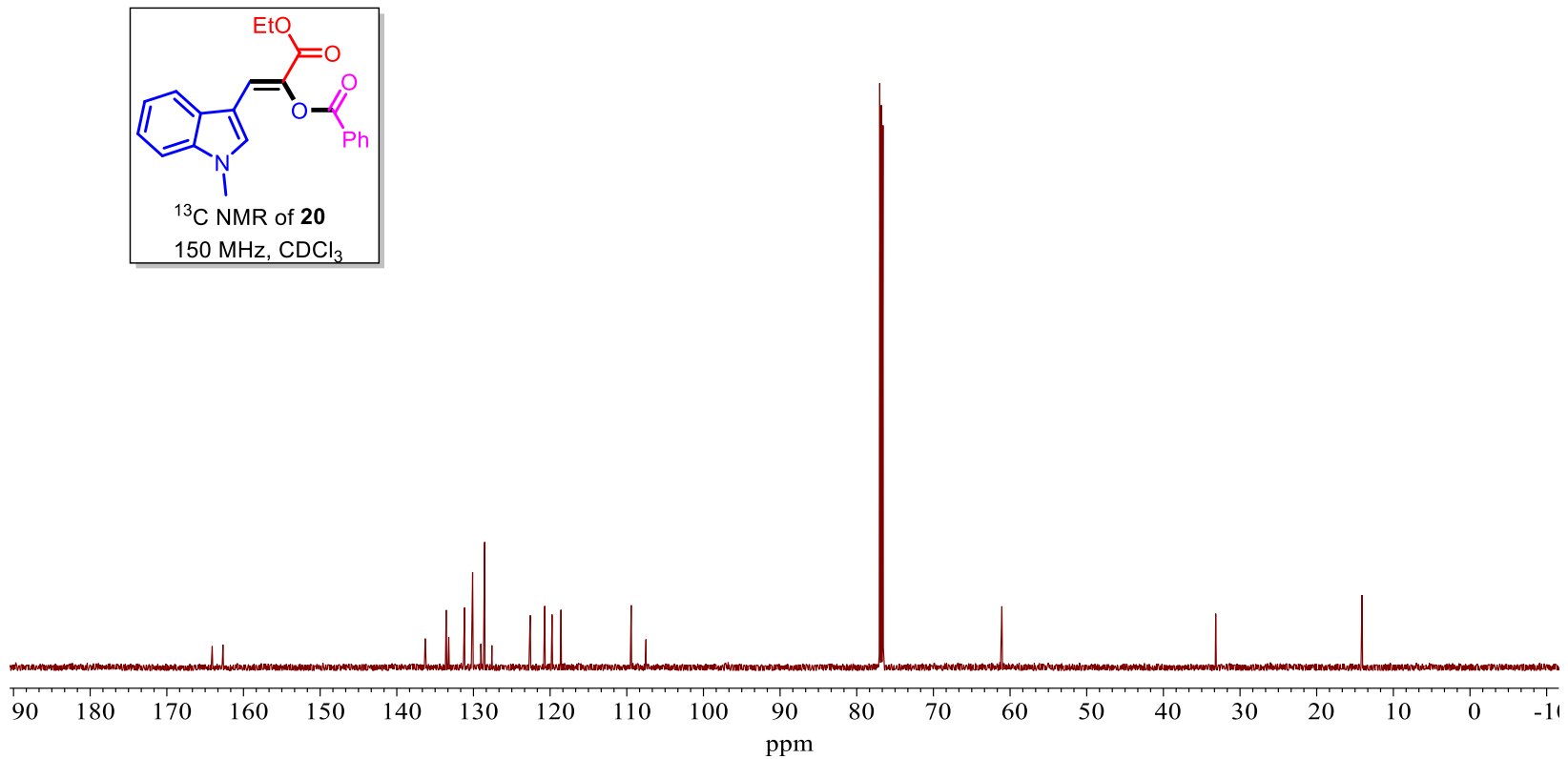

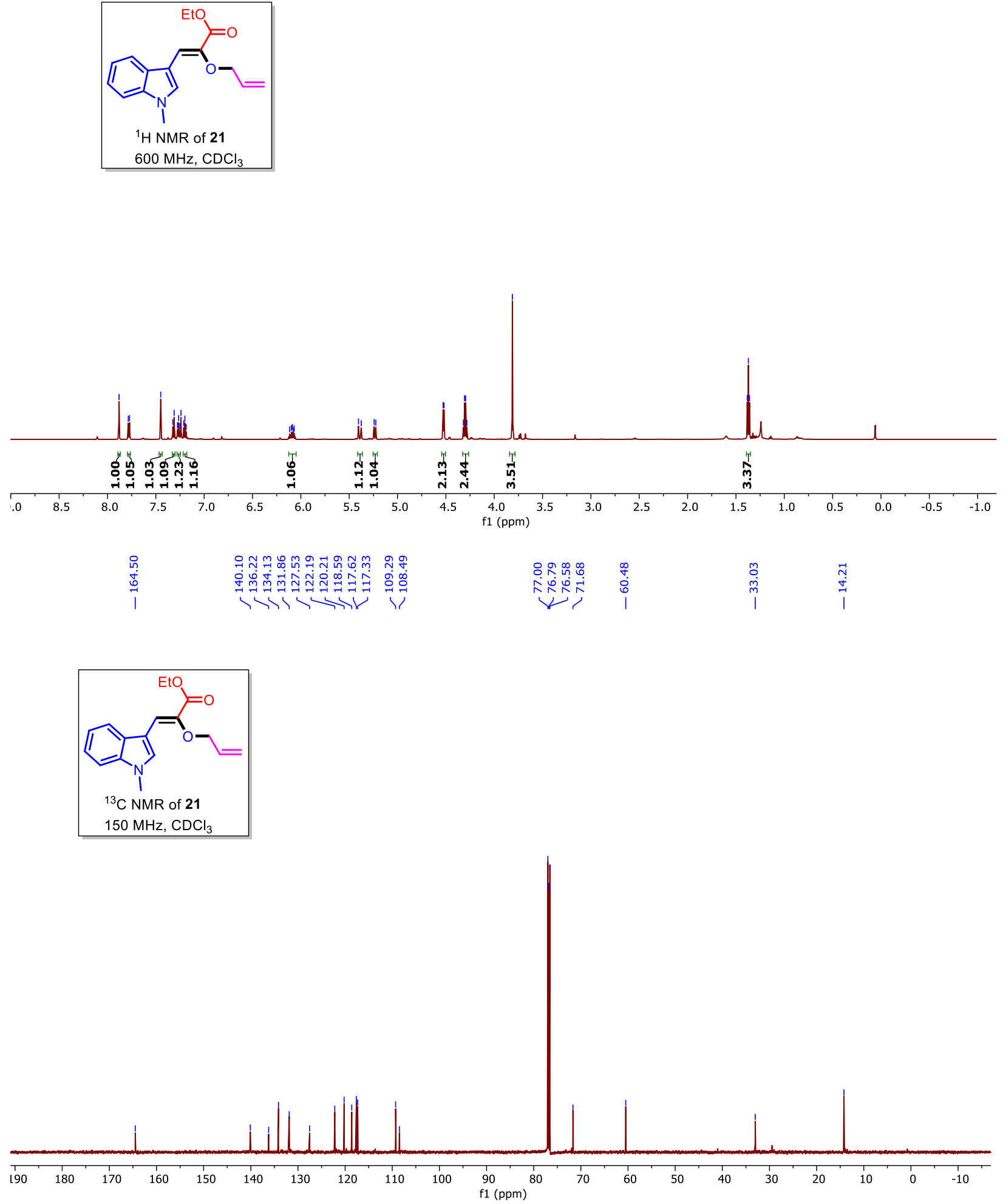


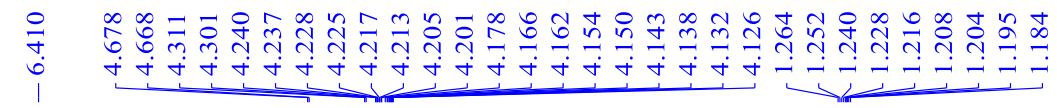

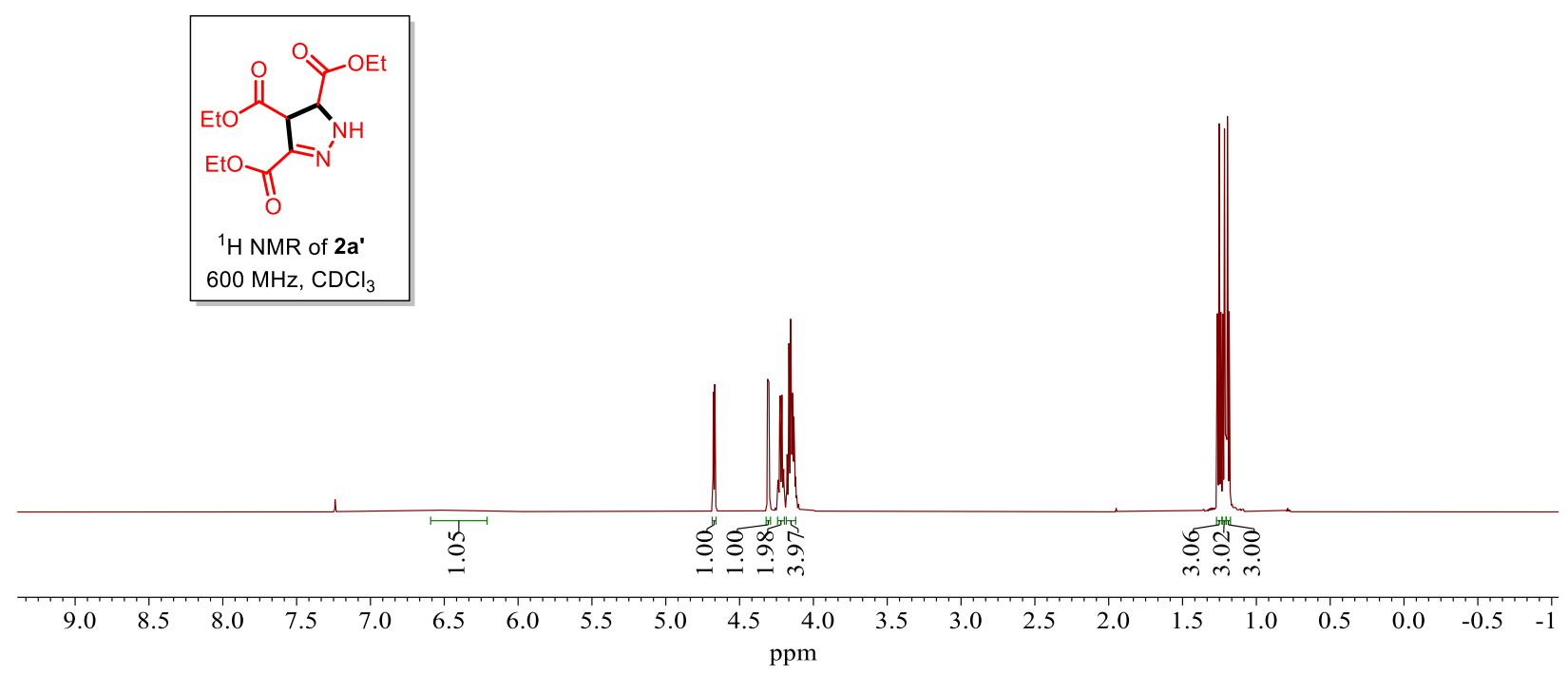
ज़
웅

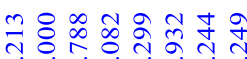
돈요영
$\overline{0} \infty \underset{\infty}{\infty}$
$\dot{m}$

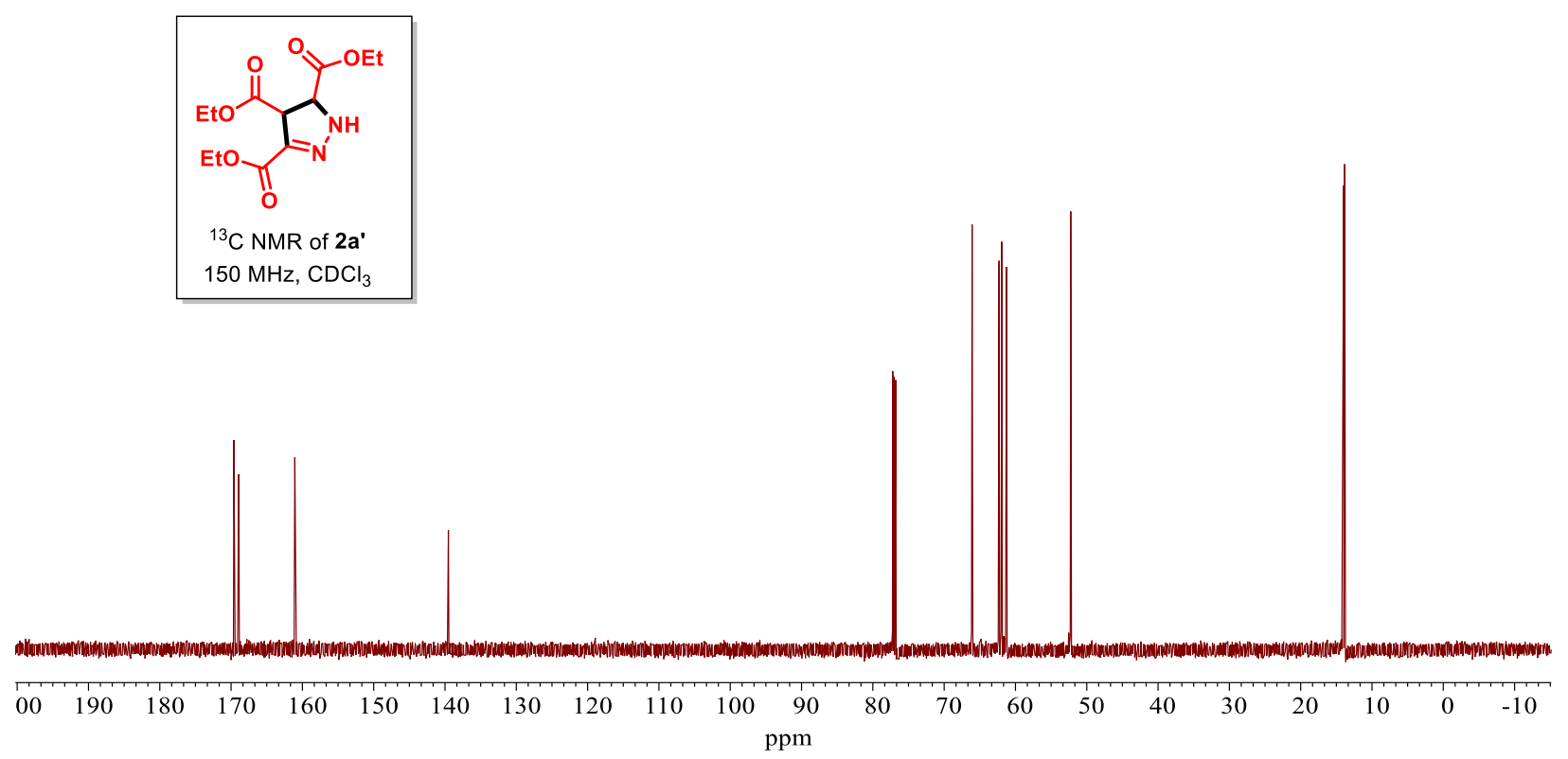




\section{References:}

1. Ann, J.; Czikora, A.; Saini, A. S.; Zhou, X.; Mitchell, G. M.; Lewin, N. E.; Peach, M. L.; Blumberg, P. M.; Lee, J. $\alpha$-Arylidene Diacylglycerol-Lactones (DAG-Lactones) as Selective Ras Guanine-Releasing Protein 3 (RasGRP3) Ligands. J. Med. Chem. 2018, 61, 6261-6276.

2. (a) Sagnes, C.; Fournet, G.; Satala, G.; Bojarski, A. J.; Joseph, B. New 1-Arylindoles Based Serotonin 5-HT7 Antagonists. Synthesis and Binding Evaluation Studies. Eur. J. Med. Chem. 2014, 75, 159-168. (b) Thrimurtulu, N.; Dey, A.; Singh, A.; Pal, K.; Maiti, D.; Volla, C. M. R. Palladium Catalyzed Regioselective C4-Arylation and Olefination of Indoles and Azaindoles. Adv. Synth. Catal. 2019, 361, 1441-1446. (c) Wang, Y.-F.; Lonca, G. H.; Runigo, M. L.; Chiba, S. Synthesis of Polyfluoroalkyl Aza-Polycyclic Aromatic Hydrocarbons Enabled by Addition of Perfluoroalkyl Radicals onto Vinyl Azides. Org. Lett. 2014, 16, 4272-4275.

3. Ideue, E.; Toma, T.; Shimokawa, J.; Fukuyama, T. Convenient Synthesis of $\alpha$-Diazoacetates from $\alpha$-Bromoacetates and $N, N^{\prime}$-Ditosylhydrazine: Preparation of Benzyl Diazoacetate. Org. Synth. 2012, 89, 501-509.

4. Downie, I. M.; Earle, M. J., Heaney, H.; Shuhaibar, K. F. Vilsmeier Formylation and Glyoxylation Reactions of Nucleophilic Aromatic Compounds Using Pyrophosphoryl Chloride. Tetrahedron, 1993, 49, 4015. 


\section{Method of crystal growth for compounds $4 \mathrm{~m}, 6 \mathrm{e}$ and $7 \mathrm{c}$ and instrumentation}

In a $5 \mathrm{~mL}$ vial, $50 \mathrm{mg}$ of the pure isolated compound was dissolved in minimum volume of ethyl acetate at room temperature. Then, $1 \mathrm{~mL}$ of hexane was added to the mixture. The mixture was shaken well to obtain a homogenous and clear solution. Then, the solution was covered with a porous cap and allowed to stand undisturbed at room temperature for 2 days until the crystal was formed via slow evaporation method.

The crystal structures of the compounds $\mathbf{4 m}, \mathbf{6 e}$ and $\mathbf{7 c}$ were determined by single crystal diffraction method at the Korea Basic Science Institute (KBSI, Western Seoul Center, Korea). Colorless block crystal $(0.262 \times 0.187 \times 0.174 \mathrm{~mm} 3)$ was picked up with paraton oil and mounted on a Bruker D8 Venture PHOTON III M14 diffractometer equipped with a graphitemonochromated Mo $\mathrm{K} \alpha(\lambda=0.71073 \AA)$ radiation source and a nitrogen cold stream $\left(-50^{\circ} \mathrm{C}\right)$. Data collection and integration were performed with SMART APEX3 (Bruker, 2016) and SAINT (Bruker, 2016) [1]. Absorption correction was performed by multi-scan method implemented in SADABS [2]. The structure was solved by direct methods and refined by fullmatrix least-squares on F2 using SHELXTL [3]. All the nonhydrogen atoms were refined anisotropically, and hydrogen atoms were added to their geometrically ideal positions.

1. SMART, SAINT and SADABS, Bruker AXS Inc., Madison, Wisconsin, USA, 2016.

2. G. M. Sheldrick, SADABS v 2.03, University of Göttingen, Germany, 2002.

3. SHELXTL v 6.10; Bruker AXS, Inc: Madison, Wisconsin, USA, 2000. 


\section{Crystal refinement data for compound $4 \mathrm{~m}$}

Empirical Formula- $\mathrm{C}_{17} \mathrm{H}_{19} \mathrm{NO}_{3}, \mathrm{M}=285.33$, Monoclinic, Space group $\mathrm{P} 2{ }_{1} / \mathrm{c}, \mathrm{a}=14.5385(13) \AA, \mathrm{b}=$ 8.4924(7) $\AA, c=13.3873(12) \AA, V=1474.0(2) \AA^{3}, Z=4, T=223(2) \mathrm{K}, \rho c a l c d=1.286 \mathrm{Mg} / \mathrm{m}^{3}, 2 \Theta_{\text {max. }}=$ $28.280^{\circ}$, Refinement of 608 parameters on 3656 independent reflections out of 23904 collected reflections $\left(\mathrm{R}_{\text {int }}=0.0401\right)$ led to $\mathrm{R} 1=0.0449[\mathrm{I}>2 \sigma(\mathrm{I})], \mathrm{wR} 2=0.1193$ (all data) and $\mathrm{S}=1.054$ with the largest difference peak and hole of 0.191 and -0.200 e. $\AA-3$ respectively. The crystal structure has been deposited at the Cambridge Crystallographic Data Centre (CCDC 2057963). The data can be obtained free of charge via the Internet at www.ccdc.cam.ac.uk/data_request/cif

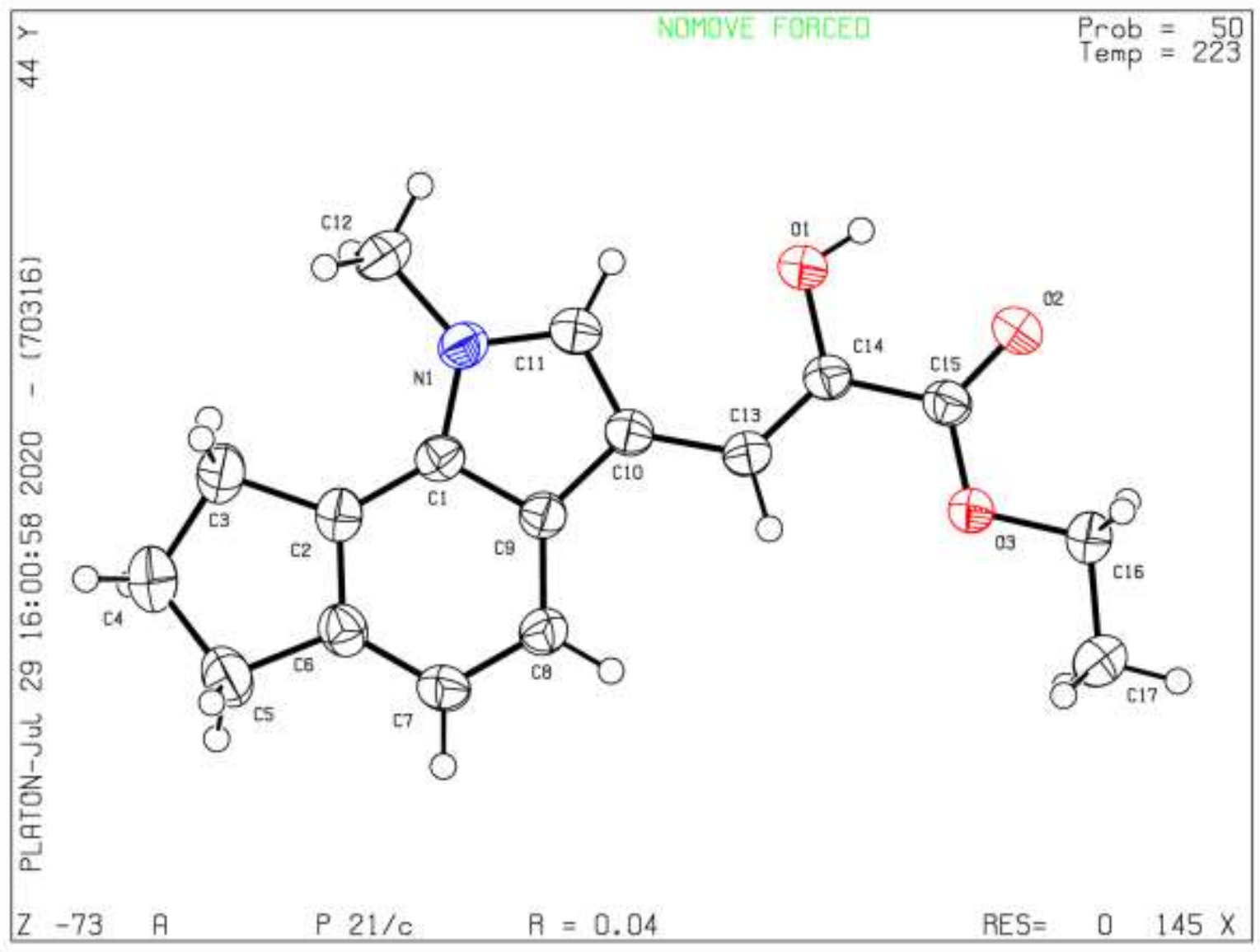


Figure S1. X-ray structure of compound $\mathbf{4 m}$ (ellipsoid contour \% probability-50).

Table 1. Crystal data and structure refinement for $\mathbf{4 m}(\mathrm{A})$.

Identification code

Empirical formula

Formula weight

Temperature

Wavelength

Crystal system

Space group

Unit cell dimensions

Volume

$\mathrm{Z}$

Density (calculated)

Absorption coefficient

$\mathrm{F}(000)$

Crystal size

Theta range for data collection

Index ranges

Reflections collected

Independent reflections

Completeness to theta $=25.242^{\circ}$

Absorption correction

Max. and min. transmission

Refinement method

Data / restraints / parameters

Goodness-of-fit on $\mathrm{F}^{2}$

Final $\mathrm{R}$ indices [I>2sigma(I)]

$\mathrm{R}$ indices (all data)

Extinction coefficient

Largest diff. peak and hole
A

C17 H19 N O3

285.33

223(2) K

$0.71073 \AA$

Monoclinic

$\mathrm{P} 2{ }_{1} / \mathrm{c}$

$\mathrm{a}=14.5385(13) \AA \quad \alpha=90^{\circ}$.

$\mathrm{b}=8.4924(7) \AA$

$\beta=116.902(3)^{\circ}$.

$\mathrm{c}=13.3873(12) \AA$ $\gamma=90^{\circ}$.

$1474.0(2) \AA^{3}$

4

$1.286 \mathrm{Mg} / \mathrm{m}^{3}$

$0.088 \mathrm{~mm}^{-1}$

608

$0.241 \times 0.130 \times 0.107 \mathrm{~mm}^{3}$

2.867 to $28.280^{\circ}$.

$-19<=\mathrm{h}<=18,-11<=\mathrm{k}<=11,-17<=1<=17$

23904

$3656[\mathrm{R}(\mathrm{int})=0.0401]$

$99.8 \%$

Semi-empirical from equivalents

0.7457 and 0.6754

Full-matrix least-squares on $\mathrm{F}^{2}$

3656 / 0 / 193

1.054

$\mathrm{R} 1=0.0449, \mathrm{wR} 2=0.1044$

$\mathrm{R} 1=0.0695, \mathrm{wR} 2=0.1193$

$\mathrm{n} / \mathrm{a}$

0.191 and -0.200 e. $\AA^{-3}$ 
Table 2. Atomic coordinates ( $\left.\mathrm{x} 10^{4}\right)$ and equivalent isotropic displacement parameters $\left(\AA^{2} \times 10^{3}\right)$ for $A$. $U(e q)$ is defined as one third of the trace of the orthogonalized $U^{i j}$ tensor.

\begin{tabular}{|c|c|c|c|c|}
\hline & $\mathrm{x}$ & $\mathrm{y}$ & $\mathrm{z}$ & $\mathrm{U}(\mathrm{eq})$ \\
\hline $\mathrm{N}(1)$ & $3826(1)$ & $3060(1)$ & $5939(1)$ & $37(1)$ \\
\hline $\mathrm{C}(1)$ & $2839(1)$ & $2536(2)$ & $5240(1)$ & $33(1)$ \\
\hline $\mathrm{C}(2)$ & 2331(1) & $1148(2)$ & $5251(1)$ & $36(1)$ \\
\hline $\mathrm{C}(3)$ & $2682(1)$ & $-260(2)$ & $6022(1)$ & $47(1)$ \\
\hline $\mathrm{C}(4)$ & $1695(2)$ & $-1235(2)$ & $5657(2)$ & $69(1)$ \\
\hline$C(5)$ & $950(1)$ & $-679(2)$ & $4485(2)$ & $52(1)$ \\
\hline $\mathrm{C}(6)$ & 1334(1) & $945(2)$ & $4409(1)$ & $39(1)$ \\
\hline$C(7)$ & $830(1)$ & $2108(2)$ & $3606(1)$ & $40(1)$ \\
\hline $\mathrm{C}(8)$ & $1326(1)$ & $3496(2)$ & $3613(1)$ & $37(1)$ \\
\hline $\mathrm{C}(9)$ & $2347(1)$ & $3718(2)$ & $4426(1)$ & $33(1)$ \\
\hline $\mathrm{C}(10)$ & $3076(1)$ & $4986(2)$ & 4664(1) & $35(1)$ \\
\hline $\mathrm{C}(11)$ & $3959(1)$ & $4513(2)$ & $5590(1)$ & $38(1)$ \\
\hline $\mathrm{C}(12)$ & $4583(1)$ & $2276(2)$ & 6937(1) & $49(1)$ \\
\hline$C(13)$ & $2898(1)$ & $6434(2)$ & $4043(1)$ & $36(1)$ \\
\hline $\mathrm{C}(14)$ & $3554(1)$ & $7648(2)$ & $4291(1)$ & $36(1)$ \\
\hline $\mathrm{O}(1)$ & $4494(1)$ & 7593(1) & $5202(1)$ & $46(1)$ \\
\hline $\mathrm{C}(15)$ & $3332(1)$ & $9118(2)$ & $3643(1)$ & $35(1)$ \\
\hline $\mathrm{O}(2)$ & $3964(1)$ & 10171(1) & $3887(1)$ & $48(1)$ \\
\hline $\mathrm{O}(3)$ & 2401(1) & 9188(1) & 2781(1) & $38(1)$ \\
\hline$C(16)$ & 2181(1) & $10612(2)$ & 2104(1) & $42(1)$ \\
\hline$C(17)$ & $1082(1)$ & $10538(2)$ & $1235(1)$ & $47(1)$ \\
\hline
\end{tabular}

Table 3. Bond lengths $[\AA]$ and angles $\left[{ }^{\circ}\right]$ for $\mathrm{A}$.

\begin{tabular}{ll}
\hline $\mathrm{N}(1)-\mathrm{C}(11)$ & $1.3637(19)$ \\
$\mathrm{N}(1)-\mathrm{C}(1)$ & $1.3845(18)$ \\
$\mathrm{N}(1)-\mathrm{C}(12)$ & $1.4532(19)$ \\
$\mathrm{C}(1)-\mathrm{C}(2)$ & $1.395(2)$ \\
$\mathrm{C}(1)-\mathrm{C}(9)$ & $1.4141(19)$
\end{tabular}




\begin{tabular}{|c|c|}
\hline$C(2)-C(6)$ & $1.387(2)$ \\
\hline$C(2)-C(3)$ & $1.509(2)$ \\
\hline$C(3)-C(4)$ & $1.532(3)$ \\
\hline $\mathrm{C}(3)-\mathrm{H}(3 \mathrm{~A})$ & 0.9800 \\
\hline $\mathrm{C}(3)-\mathrm{H}(3 \mathrm{~B})$ & 0.9800 \\
\hline$C(4)-C(5)$ & $1.524(3)$ \\
\hline $\mathrm{C}(4)-\mathrm{H}(4 \mathrm{~A})$ & 0.9800 \\
\hline $\mathrm{C}(4)-\mathrm{H}(4 \mathrm{~B})$ & 0.9800 \\
\hline$C(5)-C(6)$ & $1.508(2)$ \\
\hline $\mathrm{C}(5)-\mathrm{H}(5 \mathrm{~A})$ & 0.9800 \\
\hline $\mathrm{C}(5)-\mathrm{H}(5 \mathrm{~B})$ & 0.9800 \\
\hline$C(6)-C(7)$ & $1.399(2)$ \\
\hline $\mathrm{C}(7)-\mathrm{C}(8)$ & $1.380(2)$ \\
\hline $\mathrm{C}(7)-\mathrm{H}(7)$ & 0.9400 \\
\hline $\mathrm{C}(8)-\mathrm{C}(9)$ & 1.4011(19) \\
\hline $\mathrm{C}(8)-\mathrm{H}(8)$ & 0.9400 \\
\hline $\mathrm{C}(9)-\mathrm{C}(10)$ & $1.442(2)$ \\
\hline $\mathrm{C}(10)-\mathrm{C}(11)$ & $1.381(2)$ \\
\hline $\mathrm{C}(10)-\mathrm{C}(13)$ & $1.441(2)$ \\
\hline $\mathrm{C}(11)-\mathrm{H}(11)$ & 0.9400 \\
\hline $\mathrm{C}(12)-\mathrm{H}(12 \mathrm{~A})$ & 0.9700 \\
\hline $\mathrm{C}(12)-\mathrm{H}(12 \mathrm{~B})$ & 0.9700 \\
\hline $\mathrm{C}(12)-\mathrm{H}(12 \mathrm{C})$ & 0.9700 \\
\hline$C(13)-C(14)$ & $1.341(2)$ \\
\hline $\mathrm{C}(13)-\mathrm{H}(13)$ & 0.9400 \\
\hline $\mathrm{C}(14)-\mathrm{O}(1)$ & $1.3593(16)$ \\
\hline $\mathrm{C}(14)-\mathrm{C}(15)$ & $1.470(2)$ \\
\hline $\mathrm{O}(1)-\mathrm{H}(1)$ & 0.8300 \\
\hline $\mathrm{C}(15)-\mathrm{O}(2)$ & $1.2154(17)$ \\
\hline $\mathrm{C}(15)-\mathrm{O}(3)$ & $1.3253(17)$ \\
\hline $\mathrm{O}(3)-\mathrm{C}(16)$ & $1.4574(17)$ \\
\hline$C(16)-C(17)$ & $1.493(2)$ \\
\hline $\mathrm{C}(16)-\mathrm{H}(16 \mathrm{~A})$ & 0.9800 \\
\hline $\mathrm{C}(16)-\mathrm{H}(16 \mathrm{~B})$ & 0.9800 \\
\hline $\mathrm{C}(17)-\mathrm{H}(17 \mathrm{~A})$ & 0.9700 \\
\hline
\end{tabular}




\begin{tabular}{|c|c|}
\hline $\mathrm{C}(17)-\mathrm{H}(17 \mathrm{~B})$ & 0.9700 \\
\hline $\mathrm{C}(17)-\mathrm{H}(17 \mathrm{C})$ & 0.9700 \\
\hline $\mathrm{C}(11)-\mathrm{N}(1)-\mathrm{C}(1)$ & $108.93(12)$ \\
\hline $\mathrm{C}(11)-\mathrm{N}(1)-\mathrm{C}(12)$ & $124.29(13)$ \\
\hline $\mathrm{C}(1)-\mathrm{N}(1)-\mathrm{C}(12)$ & $126.62(13)$ \\
\hline $\mathrm{N}(1)-\mathrm{C}(1)-\mathrm{C}(2)$ & $131.02(13)$ \\
\hline $\mathrm{N}(1)-\mathrm{C}(1)-\mathrm{C}(9)$ & $107.46(12)$ \\
\hline $\mathrm{C}(2)-\mathrm{C}(1)-\mathrm{C}(9)$ & $121.51(13)$ \\
\hline$C(6)-C(2)-C(1)$ & $117.37(13)$ \\
\hline$C(6)-C(2)-C(3)$ & $111.30(13)$ \\
\hline $\mathrm{C}(1)-\mathrm{C}(2)-\mathrm{C}(3)$ & $131.31(14)$ \\
\hline $\mathrm{C}(2)-\mathrm{C}(3)-\mathrm{C}(4)$ & $103.42(14)$ \\
\hline $\mathrm{C}(2)-\mathrm{C}(3)-\mathrm{H}(3 \mathrm{~A})$ & 111.1 \\
\hline $\mathrm{C}(4)-\mathrm{C}(3)-\mathrm{H}(3 \mathrm{~A})$ & 111.1 \\
\hline $\mathrm{C}(2)-\mathrm{C}(3)-\mathrm{H}(3 \mathrm{~B})$ & 111.1 \\
\hline $\mathrm{C}(4)-\mathrm{C}(3)-\mathrm{H}(3 \mathrm{~B})$ & 111.1 \\
\hline $\mathrm{H}(3 \mathrm{~A})-\mathrm{C}(3)-\mathrm{H}(3 \mathrm{~B})$ & 109.0 \\
\hline$C(5)-C(4)-C(3)$ & $106.69(14)$ \\
\hline $\mathrm{C}(5)-\mathrm{C}(4)-\mathrm{H}(4 \mathrm{~A})$ & 110.4 \\
\hline $\mathrm{C}(3)-\mathrm{C}(4)-\mathrm{H}(4 \mathrm{~A})$ & 110.4 \\
\hline $\mathrm{C}(5)-\mathrm{C}(4)-\mathrm{H}(4 \mathrm{~B})$ & 110.4 \\
\hline $\mathrm{C}(3)-\mathrm{C}(4)-\mathrm{H}(4 \mathrm{~B})$ & 110.4 \\
\hline $\mathrm{H}(4 \mathrm{~A})-\mathrm{C}(4)-\mathrm{H}(4 \mathrm{~B})$ & 108.6 \\
\hline$C(6)-C(5)-C(4)$ & $103.93(14)$ \\
\hline $\mathrm{C}(6)-\mathrm{C}(5)-\mathrm{H}(5 \mathrm{~A})$ & 111.0 \\
\hline $\mathrm{C}(4)-\mathrm{C}(5)-\mathrm{H}(5 \mathrm{~A})$ & 111.0 \\
\hline $\mathrm{C}(6)-\mathrm{C}(5)-\mathrm{H}(5 \mathrm{~B})$ & 111.0 \\
\hline $\mathrm{C}(4)-\mathrm{C}(5)-\mathrm{H}(5 \mathrm{~B})$ & 111.0 \\
\hline $\mathrm{H}(5 \mathrm{~A})-\mathrm{C}(5)-\mathrm{H}(5 \mathrm{~B})$ & 109.0 \\
\hline$C(2)-C(6)-C(7)$ & $121.94(14)$ \\
\hline$C(2)-C(6)-C(5)$ & $109.95(14)$ \\
\hline$C(7)-C(6)-C(5)$ & $128.05(15)$ \\
\hline$C(8)-C(7)-C(6)$ & $120.50(14)$ \\
\hline $\mathrm{C}(8)-\mathrm{C}(7)-\mathrm{H}(7)$ & 119.8 \\
\hline
\end{tabular}




\begin{tabular}{|c|c|}
\hline $\mathrm{C}(6)-\mathrm{C}(7)-\mathrm{H}(7)$ & 119.8 \\
\hline $\mathrm{C}(7)-\mathrm{C}(8)-\mathrm{C}(9)$ & $119.14(13)$ \\
\hline $\mathrm{C}(7)-\mathrm{C}(8)-\mathrm{H}(8)$ & 120.4 \\
\hline $\mathrm{C}(9)-\mathrm{C}(8)-\mathrm{H}(8)$ & 120.4 \\
\hline $\mathrm{C}(8)-\mathrm{C}(9)-\mathrm{C}(1)$ & $119.47(13)$ \\
\hline $\mathrm{C}(8)-\mathrm{C}(9)-\mathrm{C}(10)$ & $133.33(13)$ \\
\hline$C(1)-C(9)-C(10)$ & $107.19(12)$ \\
\hline$C(11)-C(10)-C(13)$ & $128.15(14)$ \\
\hline $\mathrm{C}(11)-\mathrm{C}(10)-\mathrm{C}(9)$ & $105.82(13)$ \\
\hline $\mathrm{C}(13)-\mathrm{C}(10)-\mathrm{C}(9)$ & $126.03(13)$ \\
\hline $\mathrm{N}(1)-\mathrm{C}(11)-\mathrm{C}(10)$ & $110.60(13)$ \\
\hline $\mathrm{N}(1)-\mathrm{C}(11)-\mathrm{H}(11)$ & 124.7 \\
\hline $\mathrm{C}(10)-\mathrm{C}(11)-\mathrm{H}(11)$ & 124.7 \\
\hline $\mathrm{N}(1)-\mathrm{C}(12)-\mathrm{H}(12 \mathrm{~A})$ & 109.5 \\
\hline $\mathrm{N}(1)-\mathrm{C}(12)-\mathrm{H}(12 \mathrm{~B})$ & 109.5 \\
\hline $\mathrm{H}(12 \mathrm{~A})-\mathrm{C}(12)-\mathrm{H}(12 \mathrm{~B})$ & 109.5 \\
\hline $\mathrm{N}(1)-\mathrm{C}(12)-\mathrm{H}(12 \mathrm{C})$ & 109.5 \\
\hline $\mathrm{H}(12 \mathrm{~A})-\mathrm{C}(12)-\mathrm{H}(12 \mathrm{C})$ & 109.5 \\
\hline $\mathrm{H}(12 \mathrm{~B})-\mathrm{C}(12)-\mathrm{H}(12 \mathrm{C})$ & 109.5 \\
\hline$C(14)-C(13)-C(10)$ & $126.27(13)$ \\
\hline $\mathrm{C}(14)-\mathrm{C}(13)-\mathrm{H}(13)$ & 116.9 \\
\hline $\mathrm{C}(10)-\mathrm{C}(13)-\mathrm{H}(13)$ & 116.9 \\
\hline $\mathrm{C}(13)-\mathrm{C}(14)-\mathrm{O}(1)$ & $120.64(13)$ \\
\hline$C(13)-C(14)-C(15)$ & $124.43(13)$ \\
\hline $\mathrm{O}(1)-\mathrm{C}(14)-\mathrm{C}(15)$ & $114.92(12)$ \\
\hline $\mathrm{C}(14)-\mathrm{O}(1)-\mathrm{H}(1)$ & 109.5 \\
\hline $\mathrm{O}(2)-\mathrm{C}(15)-\mathrm{O}(3)$ & $123.47(13)$ \\
\hline $\mathrm{O}(2)-\mathrm{C}(15)-\mathrm{C}(14)$ & 121.93(13) \\
\hline $\mathrm{O}(3)-\mathrm{C}(15)-\mathrm{C}(14)$ & $114.61(12)$ \\
\hline $\mathrm{C}(15)-\mathrm{O}(3)-\mathrm{C}(16)$ & $115.37(11)$ \\
\hline $\mathrm{O}(3)-\mathrm{C}(16)-\mathrm{C}(17)$ & $107.81(12)$ \\
\hline $\mathrm{O}(3)-\mathrm{C}(16)-\mathrm{H}(16 \mathrm{~A})$ & 110.1 \\
\hline $\mathrm{C}(17)-\mathrm{C}(16)-\mathrm{H}(16 \mathrm{~A})$ & 110.1 \\
\hline $\mathrm{O}(3)-\mathrm{C}(16)-\mathrm{H}(16 \mathrm{~B})$ & 110.1 \\
\hline $\mathrm{C}(17)-\mathrm{C}(16)-\mathrm{H}(16 \mathrm{~B})$ & 110.1 \\
\hline
\end{tabular}




$\begin{array}{ll}\text { H(16A)-C(16)-H(16B) } & 108.5 \\ \text { C(16)-C(17)-H(17A) } & 109.5 \\ \text { C(16)-C(17)-H(17B) } & 109.5 \\ \text { H(17A)-C(17)-H(17B) } & 109.5 \\ \text { C(16)-C(17)-H(17C) } & 109.5 \\ \text { H(17A)-C(17)-H(17C) } & 109.5 \\ \text { H(17B)-C(17)-H(17C) } & 109.5\end{array}$

Symmetry transformations used to generate equivalent atoms:

Table 4. Anisotropic displacement parameters $\left(\AA^{2} \mathrm{x} 10^{3}\right)$ for $\mathrm{A}$. The anisotropic displacement factor exponent takes the form: $-2 \pi^{2}\left[h^{2} a^{* 2} U^{11}+\ldots+2 h k a^{*} b^{*} U^{12}\right]$

\begin{tabular}{|c|c|c|c|c|c|c|}
\hline & $\mathrm{U}^{11}$ & $\mathrm{U}^{22}$ & $\mathrm{U}^{33}$ & $\mathrm{U}^{23}$ & $\mathrm{U}^{13}$ & $\mathrm{U}^{12}$ \\
\hline $\mathrm{N}(1)$ & $36(1)$ & $37(1)$ & $32(1)$ & 1(1) & $10(1)$ & $2(1)$ \\
\hline $\mathrm{C}(1)$ & $35(1)$ & $34(1)$ & $29(1)$ & $-4(1)$ & $13(1)$ & $2(1)$ \\
\hline$C(2)$ & $45(1)$ & $32(1)$ & $34(1)$ & $0(1)$ & $21(1)$ & $2(1)$ \\
\hline$C(3)$ & $56(1)$ & $38(1)$ & $46(1)$ & $8(1)$ & $23(1)$ & $3(1)$ \\
\hline$C(4)$ & $68(1)$ & $55(1)$ & $76(1)$ & $23(1)$ & $26(1)$ & $-8(1)$ \\
\hline$C(5)$ & $58(1)$ & $41(1)$ & $60(1)$ & $0(1)$ & $27(1)$ & $-11(1)$ \\
\hline$C(6)$ & $45(1)$ & $35(1)$ & $38(1)$ & $-4(1)$ & $20(1)$ & $-4(1)$ \\
\hline$C(7)$ & $37(1)$ & $42(1)$ & $35(1)$ & $-5(1)$ & $11(1)$ & $-6(1)$ \\
\hline$C(8)$ & $38(1)$ & $36(1)$ & $31(1)$ & $0(1)$ & $11(1)$ & $-1(1)$ \\
\hline $\mathrm{C}(9)$ & $36(1)$ & $32(1)$ & $28(1)$ & $-2(1)$ & $13(1)$ & $-1(1)$ \\
\hline$C(10)$ & $36(1)$ & $34(1)$ & $32(1)$ & $-3(1)$ & $12(1)$ & $-3(1)$ \\
\hline $\mathrm{C}(11)$ & $36(1)$ & $39(1)$ & $35(1)$ & $-3(1)$ & $12(1)$ & $-4(1)$ \\
\hline $\mathrm{C}(12)$ & $42(1)$ & $49(1)$ & $42(1)$ & $8(1)$ & $6(1)$ & $7(1)$ \\
\hline$C(13)$ & $35(1)$ & $37(1)$ & $30(1)$ & $0(1)$ & $10(1)$ & $-2(1)$ \\
\hline$C(14)$ & $35(1)$ & $38(1)$ & $30(1)$ & $0(1)$ & $11(1)$ & $-4(1)$ \\
\hline $\mathrm{O}(1)$ & $37(1)$ & $43(1)$ & $42(1)$ & $6(1)$ & $4(1)$ & $-9(1)$ \\
\hline$C(15)$ & $34(1)$ & $36(1)$ & $33(1)$ & $-2(1)$ & $13(1)$ & $-5(1)$ \\
\hline $\mathrm{O}(2)$ & $39(1)$ & $42(1)$ & $51(1)$ & $4(1)$ & $8(1)$ & $-11(1)$ \\
\hline $\mathrm{O}(3)$ & $36(1)$ & $36(1)$ & $34(1)$ & $4(1)$ & $8(1)$ & $-6(1)$ \\
\hline$C(16)$ & $41(1)$ & $38(1)$ & $44(1)$ & $10(1)$ & $16(1)$ & $-1(1)$ \\
\hline
\end{tabular}


$\begin{array}{lllllll}\mathrm{C}(17) & 44(1) & 46(1) & 42(1) & 5(1) & 12(1) & 3(1)\end{array}$

Table 5. Hydrogen coordinates ( $\left.\mathrm{x} 10^{4}\right)$ and isotropic displacement parameters $\left(\AA^{2} \mathrm{x} 10^{3}\right)$ for A.

\begin{tabular}{|c|c|c|c|c|}
\hline & $\mathrm{x}$ & $\mathrm{y}$ & $\mathrm{z}$ & $\mathrm{U}(\mathrm{eq})$ \\
\hline $\mathrm{H}(3 \mathrm{~A})$ & 2955 & 63 & 6807 & 57 \\
\hline $\mathrm{H}(3 \mathrm{~B})$ & 3213 & -854 & 5924 & 57 \\
\hline $\mathrm{H}(4 \mathrm{~A})$ & 1845 & -2358 & 5649 & 82 \\
\hline $\mathrm{H}(4 \mathrm{~B})$ & 1396 & -1070 & 6175 & 82 \\
\hline $\mathrm{H}(5 \mathrm{~A})$ & 971 & -1378 & 3913 & 63 \\
\hline $\mathrm{H}(5 \mathrm{~B})$ & 242 & -637 & 4392 & 63 \\
\hline $\mathrm{H}(7)$ & 147 & 1944 & 3058 & 48 \\
\hline $\mathrm{H}(8)$ & 983 & 4283 & 3078 & 44 \\
\hline $\mathrm{H}(11)$ & 4568 & 5111 & 5933 & 46 \\
\hline $\mathrm{H}(12 \mathrm{~A})$ & 5244 & 2803 & 7196 & 73 \\
\hline $\mathrm{H}(12 \mathrm{~B})$ & 4651 & 1187 & 6764 & 73 \\
\hline $\mathrm{H}(12 \mathrm{C})$ & 4360 & 2316 & 7519 & 73 \\
\hline $\mathrm{H}(13)$ & 2260 & 6538 & 3402 & 43 \\
\hline $\mathrm{H}(1)$ & 4813 & 8420 & 5241 & 69 \\
\hline $\mathrm{H}(16 \mathrm{~A})$ & 2637 & 10678 & 1746 & 51 \\
\hline $\mathrm{H}(16 \mathrm{~B})$ & 2294 & 11545 & 2577 & 51 \\
\hline $\mathrm{H}(17 \mathrm{~A})$ & 983 & 9629 & 759 & 70 \\
\hline $\mathrm{H}(17 \mathrm{~B})$ & 910 & 11488 & 784 & 70 \\
\hline $\mathrm{H}(17 \mathrm{C})$ & 638 & 10451 & 1598 & 70 \\
\hline
\end{tabular}

Table 6. Torsion angles $\left[{ }^{\circ}\right]$ for A.

\begin{tabular}{lc}
\hline $\mathrm{C}(11)-\mathrm{N}(1)-\mathrm{C}(1)-\mathrm{C}(2)$ & $-179.24(15)$ \\
$\mathrm{C}(12)-\mathrm{N}(1)-\mathrm{C}(1)-\mathrm{C}(2)$ & $-3.8(3)$ \\
$\mathrm{C}(11)-\mathrm{N}(1)-\mathrm{C}(1)-\mathrm{C}(9)$ & $0.18(16)$
\end{tabular}




\begin{tabular}{|c|c|}
\hline $\mathrm{C}(12)-\mathrm{N}(1)-\mathrm{C}(1)-\mathrm{C}(9)$ & $175.59(14)$ \\
\hline $\mathrm{N}(1)-\mathrm{C}(1)-\mathrm{C}(2)-\mathrm{C}(6)$ & $-178.48(14)$ \\
\hline$C(9)-C(1)-C(2)-C(6)$ & $2.2(2)$ \\
\hline $\mathrm{N}(1)-\mathrm{C}(1)-\mathrm{C}(2)-\mathrm{C}(3)$ & $-0.3(3)$ \\
\hline$C(9)-C(1)-C(2)-C(3)$ & $-179.68(15)$ \\
\hline$C(6)-C(2)-C(3)-C(4)$ & $-9.53(19)$ \\
\hline$C(1)-C(2)-C(3)-C(4)$ & $172.23(17)$ \\
\hline$C(2)-C(3)-C(4)-C(5)$ & $19.3(2)$ \\
\hline$C(3)-C(4)-C(5)-C(6)$ & $-21.8(2)$ \\
\hline$C(1)-C(2)-C(6)-C(7)$ & $-3.1(2)$ \\
\hline$C(3)-C(2)-C(6)-C(7)$ & $178.41(14)$ \\
\hline$C(1)-C(2)-C(6)-C(5)$ & $174.24(14)$ \\
\hline$C(3)-C(2)-C(6)-C(5)$ & $-4.27(19)$ \\
\hline$C(4)-C(5)-C(6)-C(2)$ & $16.3(2)$ \\
\hline$C(4)-C(5)-C(6)-C(7)$ & $-166.58(17)$ \\
\hline$C(2)-C(6)-C(7)-C(8)$ & $1.7(2)$ \\
\hline$C(5)-C(6)-C(7)-C(8)$ & $-175.13(16)$ \\
\hline $\mathrm{C}(6)-\mathrm{C}(7)-\mathrm{C}(8)-\mathrm{C}(9)$ & $0.8(2)$ \\
\hline $\mathrm{C}(7)-\mathrm{C}(8)-\mathrm{C}(9)-\mathrm{C}(1)$ & $-1.6(2)$ \\
\hline $\mathrm{C}(7)-\mathrm{C}(8)-\mathrm{C}(9)-\mathrm{C}(10)$ & $179.60(15)$ \\
\hline $\mathrm{N}(1)-\mathrm{C}(1)-\mathrm{C}(9)-\mathrm{C}(8)$ & $-179.35(13)$ \\
\hline $\mathrm{C}(2)-\mathrm{C}(1)-\mathrm{C}(9)-\mathrm{C}(8)$ & $0.1(2)$ \\
\hline $\mathrm{N}(1)-\mathrm{C}(1)-\mathrm{C}(9)-\mathrm{C}(10)$ & $-0.28(16)$ \\
\hline $\mathrm{C}(2)-\mathrm{C}(1)-\mathrm{C}(9)-\mathrm{C}(10)$ & $179.20(13)$ \\
\hline $\mathrm{C}(8)-\mathrm{C}(9)-\mathrm{C}(10)-\mathrm{C}(11)$ & $179.16(16)$ \\
\hline $\mathrm{C}(1)-\mathrm{C}(9)-\mathrm{C}(10)-\mathrm{C}(11)$ & $0.28(16)$ \\
\hline $\mathrm{C}(8)-\mathrm{C}(9)-\mathrm{C}(10)-\mathrm{C}(13)$ & $-1.0(3)$ \\
\hline $\mathrm{C}(1)-\mathrm{C}(9)-\mathrm{C}(10)-\mathrm{C}(13)$ & $-179.93(14)$ \\
\hline $\mathrm{C}(1)-\mathrm{N}(1)-\mathrm{C}(11)-\mathrm{C}(10)$ & $0.00(17)$ \\
\hline$C(12)-N(1)-C(11)-C(10)$ & $-175.54(14)$ \\
\hline $\mathrm{C}(13)-\mathrm{C}(10)-\mathrm{C}(11)-\mathrm{N}(1)$ & $-179.96(14)$ \\
\hline $\mathrm{C}(9)-\mathrm{C}(10)-\mathrm{C}(11)-\mathrm{N}(1)$ & $-0.17(17)$ \\
\hline$C(11)-C(10)-C(13)-C(14)$ & $-3.7(3)$ \\
\hline$C(9)-C(10)-C(13)-C(14)$ & $176.60(15)$ \\
\hline$C(10)-C(13)-C(14)-O(1)$ & $0.1(2)$ \\
\hline
\end{tabular}




$\begin{array}{lc}\mathrm{C}(10)-\mathrm{C}(13)-\mathrm{C}(14)-\mathrm{C}(15) & -178.83(14) \\ \mathrm{C}(13)-\mathrm{C}(14)-\mathrm{C}(15)-\mathrm{O}(2) & -178.18(15) \\ \mathrm{O}(1)-\mathrm{C}(14)-\mathrm{C}(15)-\mathrm{O}(2) & 2.8(2) \\ \mathrm{C}(13)-\mathrm{C}(14)-\mathrm{C}(15)-\mathrm{O}(3) & 1.4(2) \\ \mathrm{O}(1)-\mathrm{C}(14)-\mathrm{C}(15)-\mathrm{O}(3) & -177.63(13) \\ \mathrm{O}(2)-\mathrm{C}(15)-\mathrm{O}(3)-\mathrm{C}(16) & 1.8(2) \\ \mathrm{C}(14)-\mathrm{C}(15)-\mathrm{O}(3)-\mathrm{C}(16) & -177.79(13) \\ \mathrm{C}(15)-\mathrm{O}(3)-\mathrm{C}(16)-\mathrm{C}(17) & -175.67(13)\end{array}$

Symmetry transformations used to generate equivalent atoms:

Table 7. Hydrogen bonds for A [ $\left.\AA^{\text {and }}{ }^{\circ}\right]$.

\begin{tabular}{lcccc}
\hline D-H...A & d(D-H) & d(H...A & $d(D \ldots A)$ & $<($ DHA $)$ \\
\hline $\mathrm{O}(1)-\mathrm{H}(1) \ldots \mathrm{O}(2) \# 1$ & 0.83 & 2.02 & $2.7649(15)$ & 149.1 \\
\hline
\end{tabular}

Symmetry transformations used to generate equivalent atoms:

$\# 1-x+1,-y+2,-z+1$ 


\section{Crystal refinement data for compound $6 e$}

Empirical Formula- $\mathrm{C}_{26} \mathrm{H}_{29} \mathrm{NO}_{5}, \mathrm{M}=435.50$, Monoclinic, Space group $\mathrm{P} 2{ }_{1} / \mathrm{n}, \mathrm{a}=8.9586(8) \AA$, $\mathrm{b}=$ 21.6849(18) $\mathrm{A}, \mathrm{c}=12.6364(10) \AA, \mathrm{V}=2401.1(3) \AA^{3}, \mathrm{Z}=4, \mathrm{~T}=223(2) \mathrm{K}, \rho c a l c d=1.205 \mathrm{Mg} / \mathrm{m}^{3}, 2 \Theta_{\text {max. }}$ $=28.359^{\circ}$, Refinement of 928 parameters on 5992 independent reflections out of 33205 collected reflections $\left(\mathrm{R}_{\text {int }}=0.0379\right)$ led to $\mathrm{R} 1=0.0466[\mathrm{I}>2 \sigma(\mathrm{I})], \mathrm{wR} 2=0.1131$ (all data) and $\mathrm{S}=1.053$ with the largest difference peak and hole of 0.213 and -0.230 e. $\AA^{-3}$ respectively. The crystal structure has been deposited at the Cambridge Crystallographic Data Centre (CCDC 2057964). The data can be obtained free of charge via the Internet at www.ccdc.cam.ac.uk/data_request/cif

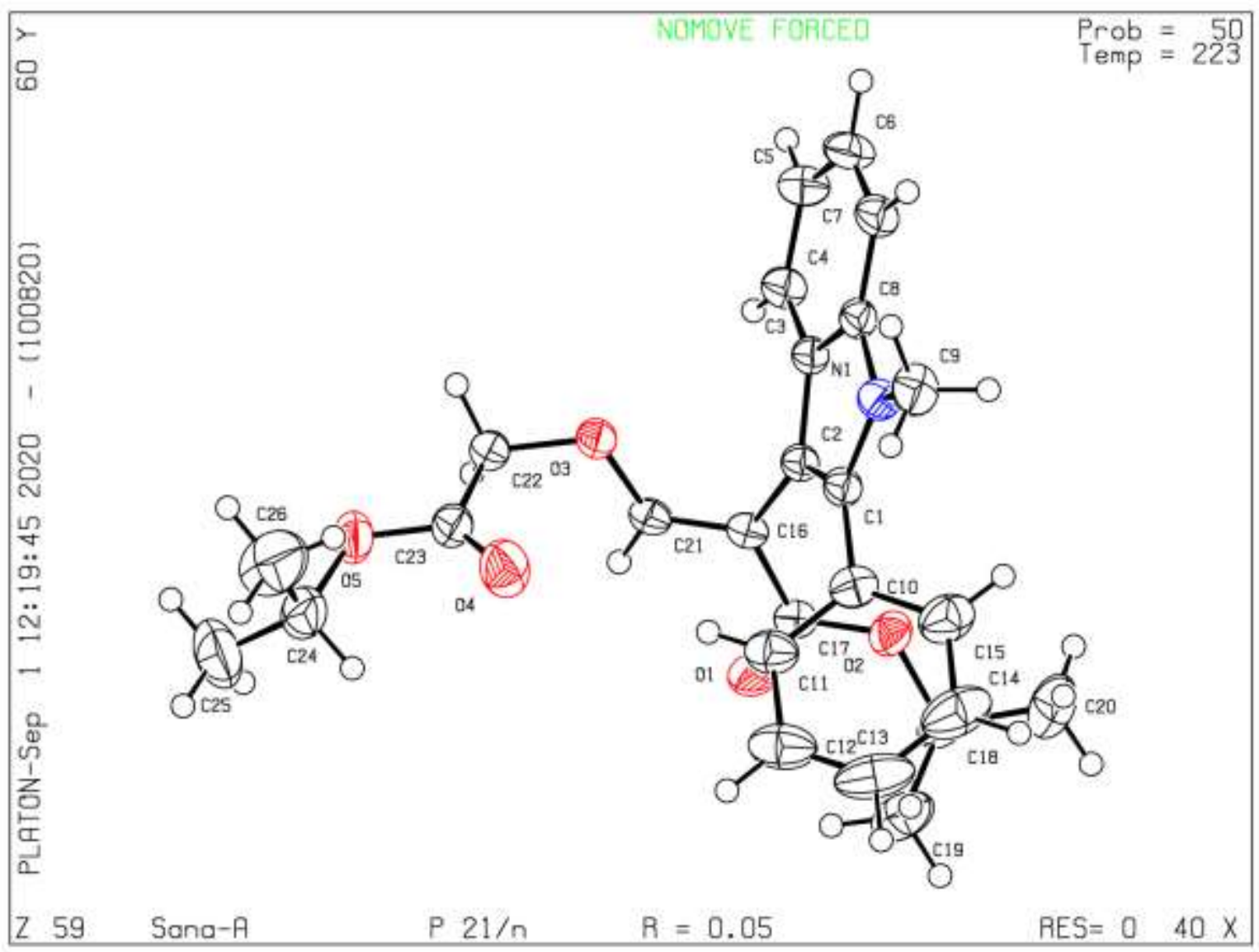

Figure S2. X-ray structure of compound 6e (ellipsoid contour \% probability-50). 
Table 1. Crystal data and structure refinement for $\mathbf{6 e}$ (Sana-A).

Identification code

Empirical formula

Formula weight

Temperature

Wavelength

Crystal system

Space group

Unit cell dimensions

Volume

Z

Density (calculated)

Absorption coefficient

$\mathrm{F}(000)$

Crystal size

Theta range for data collection

Index ranges

Reflections collected

Independent reflections

Completeness to theta $=25.242^{\circ}$

Absorption correction

Max. and min. transmission

Refinement method

Data / restraints / parameters

Goodness-of-fit on $\mathrm{F}^{2}$

Final R indices [I $>2 \operatorname{sigma}(\mathrm{I})]$

$\mathrm{R}$ indices (all data)

Extinction coefficient

Largest diff. peak and hole
Sana-A

C26 H29 N O5

435.50

223(2) K

$0.71073 \AA$

Monoclinic

$\mathrm{P} 21 / \mathrm{n}$

$\mathrm{a}=8.9586(8) \AA$

$\alpha=90^{\circ}$.

$\mathrm{b}=21.6849(18) \AA$

$\beta=102.007(2)^{\circ}$.

$\mathrm{c}=12.6364(10) \AA$

$\gamma=90^{\circ}$.
4

$1.205 \mathrm{Mg} / \mathrm{m}^{3}$

$0.083 \mathrm{~mm}^{-1}$

928

$0.315 \times 0.270 \times 0.132 \mathrm{~mm}^{3}$

1.896 to $28.359^{\circ}$.

$-11<=\mathrm{h}<=11,-28<=\mathrm{k}<=28,-12<=1<=16$

33205

$5992[\mathrm{R}(\mathrm{int})=0.0379]$

$99.9 \%$

Semi-empirical from equivalents

0.7457 and 0.6798

Full-matrix least-squares on $\mathrm{F}^{2}$

5992 / 0 / 294

1.053

$\mathrm{R} 1=0.0466, \mathrm{wR} 2=0.1019$

$\mathrm{R} 1=0.0677, \mathrm{wR} 2=0.1131$

$\mathrm{n} / \mathrm{a}$

0.213 and -0.230 e. $\AA^{-3}$ 
Table 2. Atomic coordinates ( $\left.\mathrm{x} 10^{4}\right)$ and equivalent isotropic displacement parameters $\left(\AA^{2} \times 10^{3}\right)$ for Sana-A. U(eq) is defined as one third of the trace of the orthogonalized $U^{\mathrm{ij}}$ tensor.

\begin{tabular}{|c|c|c|c|c|}
\hline & $\mathrm{x}$ & $\mathrm{y}$ & $\mathrm{z}$ & $\mathrm{U}(\mathrm{eq})$ \\
\hline $\mathrm{N}(1)$ & $5151(1)$ & $5815(1)$ & 4381(1) & $31(1)$ \\
\hline $\mathrm{C}(1)$ & $4650(2)$ & $5735(1)$ & $3274(1)$ & $28(1)$ \\
\hline $\mathrm{C}(2)$ & 3092(2) & $5656(1)$ & $3042(1)$ & $27(1)$ \\
\hline $\mathrm{C}(3)$ & $2594(2)$ & $5691(1)$ & $4049(1)$ & $28(1)$ \\
\hline $\mathrm{C}(4)$ & 1172(2) & $5657(1)$ & $4335(1)$ & $34(1)$ \\
\hline$C(5)$ & $1106(2)$ & $5699(1)$ & $5414(1)$ & $42(1)$ \\
\hline$C(6)$ & 2433(2) & $5772(1)$ & $6214(1)$ & $43(1)$ \\
\hline $\mathrm{C}(7)$ & $3849(2)$ & $5813(1)$ & $5958(1)$ & $37(1)$ \\
\hline $\mathrm{C}(8)$ & $3912(2)$ & $5780(1)$ & $4867(1)$ & $30(1)$ \\
\hline $\mathrm{C}(9)$ & $6720(2)$ & $5822(1)$ & 4976(1) & $41(1)$ \\
\hline$C(10)$ & $5719(2)$ & $5751(1)$ & 2531(1) & $34(1)$ \\
\hline $\mathrm{C}(11)$ & $5673(2)$ & $5293(1)$ & $1755(1)$ & $41(1)$ \\
\hline $\mathrm{C}(12)$ & $6668(2)$ & $5313(1)$ & $1047(1)$ & $56(1)$ \\
\hline $\mathrm{C}(13)$ & $7714(2)$ & $5781(1)$ & $1112(2)$ & $64(1)$ \\
\hline $\mathrm{C}(14)$ & $7768(2)$ & $6236(1)$ & 1873(2) & $65(1)$ \\
\hline $\mathrm{C}(15)$ & $6768(2)$ & $6226(1)$ & $2575(1)$ & $51(1)$ \\
\hline$C(16)$ & 2095(2) & $5521(1)$ & 1983(1) & $27(1)$ \\
\hline $\mathrm{C}(17)$ & 1934(2) & 5931(1) & 1034(1) & $30(1)$ \\
\hline $\mathrm{O}(1)$ & 1088(1) & $5832(1)$ & $167(1)$ & $44(1)$ \\
\hline $\mathrm{O}(2)$ & 2833(1) & $6427(1)$ & $1222(1)$ & $38(1)$ \\
\hline $\mathrm{C}(18)$ & $2867(2)$ & $6834(1)$ & $302(1)$ & $44(1)$ \\
\hline$C(19)$ & $3778(2)$ & $6540(1)$ & $-440(2)$ & $63(1)$ \\
\hline$C(20)$ & $3560(3)$ & $7425(1)$ & 794(2) & $81(1)$ \\
\hline$C(21)$ & $1270(2)$ & 5003(1) & 1812(1) & $29(1)$ \\
\hline $\mathrm{O}(3)$ & $1295(1)$ & $4583(1)$ & $2607(1)$ & $34(1)$ \\
\hline$C(22)$ & $763(2)$ & $3987(1)$ & $2236(1)$ & $33(1)$ \\
\hline$C(23)$ & 1934(2) & $3655(1)$ & $1746(1)$ & $35(1)$ \\
\hline $\mathrm{O}(4)$ & 3153(1) & $3860(1)$ & 1687(1) & $56(1)$ \\
\hline $\mathrm{O}(5)$ & 1391(1) & $3108(1)$ & $1378(1)$ & $47(1)$ \\
\hline$C(24)$ & $2347(2)$ & 2733(1) & $813(2)$ & $56(1)$ \\
\hline
\end{tabular}




$\begin{array}{lllrr}\mathrm{C}(25) & 1247(3) & 2372(1) & -16(2) & 87(1) \\ \mathrm{C}(26) & 3411(3) & 2346(1) & 1627(2) & 86(1)\end{array}$

Table 3. Bond lengths $[\AA ̊]$ and angles $\left[{ }^{\circ}\right]$ for Sana-A.

\begin{tabular}{ll}
\hline $\mathrm{N}(1)-\mathrm{C}(8)$ & $1.3779(18)$ \\
$\mathrm{N}(1)-\mathrm{C}(1)$ & $1.3887(16)$ \\
$\mathrm{N}(1)-\mathrm{C}(9)$ & $1.4496(18)$ \\
$\mathrm{C}(1)-\mathrm{C}(2)$ & $1.3764(18)$ \\
$\mathrm{C}(1)-\mathrm{C}(10)$ & $1.4739(19)$ \\
$\mathrm{C}(2)-\mathrm{C}(3)$ & $1.4358(18)$ \\
$\mathrm{C}(2)-\mathrm{C}(16)$ & $1.4748(17)$ \\
$\mathrm{C}(3)-\mathrm{C}(4)$ & $1.397(2)$ \\
$\mathrm{C}(3)-\mathrm{C}(8)$ & $1.4106(19)$ \\
$\mathrm{C}(4)-\mathrm{C}(5)$ & $1.381(2)$ \\
$\mathrm{C}(4)-\mathrm{H}(4)$ & 0.9400 \\
$\mathrm{C}(5)-\mathrm{C}(6)$ & $1.400(2)$ \\
$\mathrm{C}(5)-\mathrm{H}(5)$ & 0.9400 \\
$\mathrm{C}(6)-\mathrm{C}(7)$ & $1.375(2)$ \\
$\mathrm{C}(6)-\mathrm{H}(6)$ & 0.9400 \\
$\mathrm{C}(7)-\mathrm{C}(8)$ & $1.3937(19)$ \\
$\mathrm{C}(7)-\mathrm{H}(7)$ & 0.9400 \\
$\mathrm{C}(9)-\mathrm{H}(9 \mathrm{~A})$ & 0.9700 \\
$\mathrm{C}(9)-\mathrm{H}(9 \mathrm{~B})$ & 0.9700 \\
$\mathrm{C}(9)-\mathrm{H}(9 \mathrm{C})$ & 0.9700 \\
$\mathrm{C}(10)-\mathrm{C}(15)$ & $1.388(2)$ \\
$\mathrm{C}(10)-\mathrm{C}(11)$ & $1.391(2)$ \\
$\mathrm{C}(11)-\mathrm{C}(12)$ & $1.389(2)$ \\
$\mathrm{C}(11)-\mathrm{H}(11)$ & 0.9400 \\
$\mathrm{C}(12)-\mathrm{C}(13)$ & $1.372(3)$ \\
$\mathrm{C}(12)-\mathrm{H}(12)$ & 0.9400 \\
$\mathrm{C}(13)-\mathrm{C}(14)$ & $1.373(3)$ \\
$\mathrm{C}(13)-\mathrm{H}(13)$ & 0.9400 \\
$\mathrm{C}(14)-\mathrm{C}(15)$ & $1.385(2)$ \\
& \\
& \\
&
\end{tabular}




\begin{tabular}{|c|c|}
\hline $\mathrm{C}(14)-\mathrm{H}(14)$ & 0.9400 \\
\hline $\mathrm{C}(15)-\mathrm{H}(15)$ & 0.9400 \\
\hline$C(16)-C(21)$ & $1.3370(19)$ \\
\hline$C(16)-C(17)$ & $1.4771(18)$ \\
\hline $\mathrm{C}(17)-\mathrm{O}(1)$ & $1.2139(16)$ \\
\hline $\mathrm{C}(17)-\mathrm{O}(2)$ & $1.3343(17)$ \\
\hline $\mathrm{O}(2)-\mathrm{C}(18)$ & $1.4648(17)$ \\
\hline$C(18)-C(20)$ & $1.500(3)$ \\
\hline$C(18)-C(19)$ & $1.507(2)$ \\
\hline $\mathrm{C}(18)-\mathrm{H}(18)$ & 0.9900 \\
\hline $\mathrm{C}(19)-\mathrm{H}(19 \mathrm{~A})$ & 0.9700 \\
\hline $\mathrm{C}(19)-\mathrm{H}(19 \mathrm{~B})$ & 0.9700 \\
\hline $\mathrm{C}(19)-\mathrm{H}(19 \mathrm{C})$ & 0.9700 \\
\hline $\mathrm{C}(20)-\mathrm{H}(20 \mathrm{~A})$ & 0.9700 \\
\hline $\mathrm{C}(20)-\mathrm{H}(20 \mathrm{~B})$ & 0.9700 \\
\hline $\mathrm{C}(20)-\mathrm{H}(20 \mathrm{C})$ & 0.9700 \\
\hline $\mathrm{C}(21)-\mathrm{O}(3)$ & $1.3530(16)$ \\
\hline $\mathrm{C}(21)-\mathrm{H}(21)$ & 0.9400 \\
\hline $\mathrm{O}(3)-\mathrm{C}(22)$ & $1.4227(16)$ \\
\hline$C(22)-C(23)$ & $1.507(2)$ \\
\hline $\mathrm{C}(22)-\mathrm{H}(22 \mathrm{~A})$ & 0.9800 \\
\hline $\mathrm{C}(22)-\mathrm{H}(22 \mathrm{~B})$ & 0.9800 \\
\hline $\mathrm{C}(23)-\mathrm{O}(4)$ & $1.1961(18)$ \\
\hline $\mathrm{C}(23)-\mathrm{O}(5)$ & $1.3277(17)$ \\
\hline $\mathrm{O}(5)-\mathrm{C}(24)$ & $1.470(2)$ \\
\hline $\mathrm{C}(24)-\mathrm{C}(25)$ & $1.500(3)$ \\
\hline$C(24)-C(26)$ & $1.504(3)$ \\
\hline $\mathrm{C}(24)-\mathrm{H}(24)$ & 0.9900 \\
\hline $\mathrm{C}(25)-\mathrm{H}(25 \mathrm{~A})$ & 0.9700 \\
\hline $\mathrm{C}(25)-\mathrm{H}(25 \mathrm{~B})$ & 0.9700 \\
\hline $\mathrm{C}(25)-\mathrm{H}(25 \mathrm{C})$ & 0.9700 \\
\hline $\mathrm{C}(26)-\mathrm{H}(26 \mathrm{~A})$ & 0.9700 \\
\hline $\mathrm{C}(26)-\mathrm{H}(26 \mathrm{~B})$ & 0.9700 \\
\hline $\mathrm{C}(26)-\mathrm{H}(26 \mathrm{C})$ & 0.9700 \\
\hline
\end{tabular}




\begin{tabular}{|c|c|}
\hline $\mathrm{C}(8)-\mathrm{N}(1)-\mathrm{C}(1)$ & $108.73(11)$ \\
\hline $\mathrm{C}(8)-\mathrm{N}(1)-\mathrm{C}(9)$ & $123.62(12)$ \\
\hline $\mathrm{C}(1)-\mathrm{N}(1)-\mathrm{C}(9)$ & $126.75(12)$ \\
\hline $\mathrm{C}(2)-\mathrm{C}(1)-\mathrm{N}(1)$ & $109.30(12)$ \\
\hline $\mathrm{C}(2)-\mathrm{C}(1)-\mathrm{C}(10)$ & $129.20(12)$ \\
\hline $\mathrm{N}(1)-\mathrm{C}(1)-\mathrm{C}(10)$ & $121.49(12)$ \\
\hline$C(1)-C(2)-C(3)$ & $107.03(11)$ \\
\hline $\mathrm{C}(1)-\mathrm{C}(2)-\mathrm{C}(16)$ & $127.84(12)$ \\
\hline$C(3)-C(2)-C(16)$ & $125.02(12)$ \\
\hline $\mathrm{C}(4)-\mathrm{C}(3)-\mathrm{C}(8)$ & $119.18(12)$ \\
\hline$C(4)-C(3)-C(2)$ & $133.96(12)$ \\
\hline $\mathrm{C}(8)-\mathrm{C}(3)-\mathrm{C}(2)$ & $106.86(12)$ \\
\hline $\mathrm{C}(5)-\mathrm{C}(4)-\mathrm{C}(3)$ & $118.73(14)$ \\
\hline $\mathrm{C}(5)-\mathrm{C}(4)-\mathrm{H}(4)$ & 120.6 \\
\hline $\mathrm{C}(3)-\mathrm{C}(4)-\mathrm{H}(4)$ & 120.6 \\
\hline$C(4)-C(5)-C(6)$ & $121.05(14)$ \\
\hline $\mathrm{C}(4)-\mathrm{C}(5)-\mathrm{H}(5)$ & 119.5 \\
\hline $\mathrm{C}(6)-\mathrm{C}(5)-\mathrm{H}(5)$ & 119.5 \\
\hline$C(7)-C(6)-C(5)$ & 121.61(13) \\
\hline $\mathrm{C}(7)-\mathrm{C}(6)-\mathrm{H}(6)$ & 119.2 \\
\hline $\mathrm{C}(5)-\mathrm{C}(6)-\mathrm{H}(6)$ & 119.2 \\
\hline $\mathrm{C}(6)-\mathrm{C}(7)-\mathrm{C}(8)$ & $117.27(14)$ \\
\hline $\mathrm{C}(6)-\mathrm{C}(7)-\mathrm{H}(7)$ & 121.4 \\
\hline $\mathrm{C}(8)-\mathrm{C}(7)-\mathrm{H}(7)$ & 121.4 \\
\hline $\mathrm{N}(1)-\mathrm{C}(8)-\mathrm{C}(7)$ & $129.81(13)$ \\
\hline $\mathrm{N}(1)-\mathrm{C}(8)-\mathrm{C}(3)$ & $108.06(11)$ \\
\hline $\mathrm{C}(7)-\mathrm{C}(8)-\mathrm{C}(3)$ & $122.13(13)$ \\
\hline $\mathrm{N}(1)-\mathrm{C}(9)-\mathrm{H}(9 \mathrm{~A})$ & 109.5 \\
\hline $\mathrm{N}(1)-\mathrm{C}(9)-\mathrm{H}(9 \mathrm{~B})$ & 109.5 \\
\hline $\mathrm{H}(9 \mathrm{~A})-\mathrm{C}(9)-\mathrm{H}(9 \mathrm{~B})$ & 109.5 \\
\hline $\mathrm{N}(1)-\mathrm{C}(9)-\mathrm{H}(9 \mathrm{C})$ & 109.5 \\
\hline $\mathrm{H}(9 \mathrm{~A})-\mathrm{C}(9)-\mathrm{H}(9 \mathrm{C})$ & 109.5 \\
\hline $\mathrm{H}(9 \mathrm{~B})-\mathrm{C}(9)-\mathrm{H}(9 \mathrm{C})$ & 109.5 \\
\hline $\mathrm{C}(15)-\mathrm{C}(10)-\mathrm{C}(11)$ & $118.64(15)$ \\
\hline$C(15)-C(10)-C(1)$ & $121.12(14)$ \\
\hline
\end{tabular}




\begin{tabular}{|c|c|}
\hline$C(11)-C(10)-C(1)$ & $120.23(13)$ \\
\hline$C(12)-C(11)-C(10)$ & $120.14(17)$ \\
\hline $\mathrm{C}(12)-\mathrm{C}(11)-\mathrm{H}(11)$ & 119.9 \\
\hline $\mathrm{C}(10)-\mathrm{C}(11)-\mathrm{H}(11)$ & 119.9 \\
\hline$C(13)-C(12)-C(11)$ & $120.49(18)$ \\
\hline $\mathrm{C}(13)-\mathrm{C}(12)-\mathrm{H}(12)$ & 119.8 \\
\hline $\mathrm{C}(11)-\mathrm{C}(12)-\mathrm{H}(12)$ & 119.8 \\
\hline$C(12)-C(13)-C(14)$ & $119.85(17)$ \\
\hline $\mathrm{C}(12)-\mathrm{C}(13)-\mathrm{H}(13)$ & 120.1 \\
\hline $\mathrm{C}(14)-\mathrm{C}(13)-\mathrm{H}(13)$ & 120.1 \\
\hline$C(13)-C(14)-C(15)$ & $120.27(19)$ \\
\hline $\mathrm{C}(13)-\mathrm{C}(14)-\mathrm{H}(14)$ & 119.9 \\
\hline $\mathrm{C}(15)-\mathrm{C}(14)-\mathrm{H}(14)$ & 119.9 \\
\hline$C(14)-C(15)-C(10)$ & $120.60(18)$ \\
\hline $\mathrm{C}(14)-\mathrm{C}(15)-\mathrm{H}(15)$ & 119.7 \\
\hline $\mathrm{C}(10)-\mathrm{C}(15)-\mathrm{H}(15)$ & 119.7 \\
\hline$C(21)-C(16)-C(2)$ & $121.59(12)$ \\
\hline $\mathrm{C}(21)-\mathrm{C}(16)-\mathrm{C}(17)$ & $114.67(11)$ \\
\hline$C(2)-C(16)-C(17)$ & $123.74(12)$ \\
\hline $\mathrm{O}(1)-\mathrm{C}(17)-\mathrm{O}(2)$ & $122.94(12)$ \\
\hline $\mathrm{O}(1)-\mathrm{C}(17)-\mathrm{C}(16)$ & $124.30(13)$ \\
\hline $\mathrm{O}(2)-\mathrm{C}(17)-\mathrm{C}(16)$ & $112.76(11)$ \\
\hline $\mathrm{C}(17)-\mathrm{O}(2)-\mathrm{C}(18)$ & $117.07(11)$ \\
\hline $\mathrm{O}(2)-\mathrm{C}(18)-\mathrm{C}(20)$ & $105.15(14)$ \\
\hline $\mathrm{O}(2)-\mathrm{C}(18)-\mathrm{C}(19)$ & $109.97(13)$ \\
\hline $\mathrm{C}(20)-\mathrm{C}(18)-\mathrm{C}(19)$ & $112.82(17)$ \\
\hline $\mathrm{O}(2)-\mathrm{C}(18)-\mathrm{H}(18)$ & 109.6 \\
\hline $\mathrm{C}(20)-\mathrm{C}(18)-\mathrm{H}(18)$ & 109.6 \\
\hline $\mathrm{C}(19)-\mathrm{C}(18)-\mathrm{H}(18)$ & 109.6 \\
\hline $\mathrm{C}(18)-\mathrm{C}(19)-\mathrm{H}(19 \mathrm{~A})$ & 109.5 \\
\hline $\mathrm{C}(18)-\mathrm{C}(19)-\mathrm{H}(19 \mathrm{~B})$ & 109.5 \\
\hline H(19A)-C(19)-H(19B) & 109.5 \\
\hline $\mathrm{C}(18)-\mathrm{C}(19)-\mathrm{H}(19 \mathrm{C})$ & 109.5 \\
\hline H(19A)-C(19)-H(19C) & 109.5 \\
\hline $\mathrm{H}(19 \mathrm{~B})-\mathrm{C}(19)-\mathrm{H}(19 \mathrm{C})$ & 109.5 \\
\hline
\end{tabular}




\begin{tabular}{|c|c|}
\hline $\mathrm{C}(18)-\mathrm{C}(20)-\mathrm{H}(20 \mathrm{~A})$ & 109.5 \\
\hline $\mathrm{C}(18)-\mathrm{C}(20)-\mathrm{H}(20 \mathrm{~B})$ & 109.5 \\
\hline $\mathrm{H}(20 \mathrm{~A})-\mathrm{C}(20)-\mathrm{H}(20 \mathrm{~B})$ & 109.5 \\
\hline $\mathrm{C}(18)-\mathrm{C}(20)-\mathrm{H}(20 \mathrm{C})$ & 109.5 \\
\hline $\mathrm{H}(20 \mathrm{~A})-\mathrm{C}(20)-\mathrm{H}(20 \mathrm{C})$ & 109.5 \\
\hline $\mathrm{H}(20 \mathrm{~B})-\mathrm{C}(20)-\mathrm{H}(20 \mathrm{C})$ & 109.5 \\
\hline $\mathrm{C}(16)-\mathrm{C}(21)-\mathrm{O}(3)$ & $121.49(12)$ \\
\hline $\mathrm{C}(16)-\mathrm{C}(21)-\mathrm{H}(21)$ & 119.3 \\
\hline $\mathrm{O}(3)-\mathrm{C}(21)-\mathrm{H}(21)$ & 119.3 \\
\hline $\mathrm{C}(21)-\mathrm{O}(3)-\mathrm{C}(22)$ & $114.49(10)$ \\
\hline $\mathrm{O}(3)-\mathrm{C}(22)-\mathrm{C}(23)$ & $110.79(12)$ \\
\hline $\mathrm{O}(3)-\mathrm{C}(22)-\mathrm{H}(22 \mathrm{~A})$ & 109.5 \\
\hline $\mathrm{C}(23)-\mathrm{C}(22)-\mathrm{H}(22 \mathrm{~A})$ & 109.5 \\
\hline $\mathrm{O}(3)-\mathrm{C}(22)-\mathrm{H}(22 \mathrm{~B})$ & 109.5 \\
\hline $\mathrm{C}(23)-\mathrm{C}(22)-\mathrm{H}(22 \mathrm{~B})$ & 109.5 \\
\hline $\mathrm{H}(22 \mathrm{~A})-\mathrm{C}(22)-\mathrm{H}(22 \mathrm{~B})$ & 108.1 \\
\hline $\mathrm{O}(4)-\mathrm{C}(23)-\mathrm{O}(5)$ & $125.65(15)$ \\
\hline $\mathrm{O}(4)-\mathrm{C}(23)-\mathrm{C}(22)$ & $124.81(14)$ \\
\hline $\mathrm{O}(5)-\mathrm{C}(23)-\mathrm{C}(22)$ & $109.53(12)$ \\
\hline $\mathrm{C}(23)-\mathrm{O}(5)-\mathrm{C}(24)$ & $117.19(13)$ \\
\hline $\mathrm{O}(5)-\mathrm{C}(24)-\mathrm{C}(25)$ & $105.31(16)$ \\
\hline $\mathrm{O}(5)-\mathrm{C}(24)-\mathrm{C}(26)$ & $109.06(16)$ \\
\hline $\mathrm{C}(25)-\mathrm{C}(24)-\mathrm{C}(26)$ & $114.71(19)$ \\
\hline $\mathrm{O}(5)-\mathrm{C}(24)-\mathrm{H}(24)$ & 109.2 \\
\hline $\mathrm{C}(25)-\mathrm{C}(24)-\mathrm{H}(24)$ & 109.2 \\
\hline $\mathrm{C}(26)-\mathrm{C}(24)-\mathrm{H}(24)$ & 109.2 \\
\hline $\mathrm{C}(24)-\mathrm{C}(25)-\mathrm{H}(25 \mathrm{~A})$ & 109.5 \\
\hline $\mathrm{C}(24)-\mathrm{C}(25)-\mathrm{H}(25 \mathrm{~B})$ & 109.5 \\
\hline $\mathrm{H}(25 \mathrm{~A})-\mathrm{C}(25)-\mathrm{H}(25 \mathrm{~B})$ & 109.5 \\
\hline $\mathrm{C}(24)-\mathrm{C}(25)-\mathrm{H}(25 \mathrm{C})$ & 109.5 \\
\hline $\mathrm{H}(25 \mathrm{~A})-\mathrm{C}(25)-\mathrm{H}(25 \mathrm{C})$ & 109.5 \\
\hline $\mathrm{H}(25 \mathrm{~B})-\mathrm{C}(25)-\mathrm{H}(25 \mathrm{C})$ & 109.5 \\
\hline $\mathrm{C}(24)-\mathrm{C}(26)-\mathrm{H}(26 \mathrm{~A})$ & 109.5 \\
\hline $\mathrm{C}(24)-\mathrm{C}(26)-\mathrm{H}(26 \mathrm{~B})$ & 109.5 \\
\hline $\mathrm{H}(26 \mathrm{~A})-\mathrm{C}(26)-\mathrm{H}(26 \mathrm{~B})$ & 109.5 \\
\hline
\end{tabular}


$\mathrm{C}(24)-\mathrm{C}(26)-\mathrm{H}(26 \mathrm{C}) \quad 109.5$

$\mathrm{H}(26 \mathrm{~A})-\mathrm{C}(26)-\mathrm{H}(26 \mathrm{C}) \quad 109.5$

$\mathrm{H}(26 \mathrm{~B})-\mathrm{C}(26)-\mathrm{H}(26 \mathrm{C}) \quad 109.5$

Symmetry transformations used to generate equivalent atoms:

Table 4. Anisotropic displacement parameters $\left(\AA^{2} \mathrm{x} 10^{3}\right)$ for Sana-A. The anisotropic displacement factor exponent takes the form: $-2 \pi^{2}\left[h^{2} a^{* 2} U^{11}+\ldots+2 h k a^{*} b^{*} U^{12}\right]$

\begin{tabular}{|c|c|c|c|c|c|c|}
\hline & $\mathrm{U}^{11}$ & $\mathrm{U}^{22}$ & $\mathrm{U}^{33}$ & $\mathrm{U}^{23}$ & $\mathrm{U}^{13}$ & $\mathrm{U}^{12}$ \\
\hline $\mathrm{N}(1)$ & $30(1)$ & $35(1)$ & $25(1)$ & $0(1)$ & $-1(1)$ & $-2(1)$ \\
\hline $\mathrm{C}(1)$ & $31(1)$ & $28(1)$ & $25(1)$ & $0(1)$ & $2(1)$ & $-1(1)$ \\
\hline$C(2)$ & $29(1)$ & $26(1)$ & $23(1)$ & $0(1)$ & $4(1)$ & $0(1)$ \\
\hline$C(3)$ & $33(1)$ & $25(1)$ & $24(1)$ & $0(1)$ & $4(1)$ & $-1(1)$ \\
\hline$C(4)$ & $35(1)$ & $38(1)$ & $30(1)$ & $-1(1)$ & $6(1)$ & $0(1)$ \\
\hline$C(5)$ & $45(1)$ & $49(1)$ & $35(1)$ & $-1(1)$ & $16(1)$ & $2(1)$ \\
\hline$C(6)$ & $58(1)$ & $49(1)$ & $25(1)$ & $-2(1)$ & $13(1)$ & $1(1)$ \\
\hline$C(7)$ & $47(1)$ & $37(1)$ & $25(1)$ & $-2(1)$ & $1(1)$ & $-3(1)$ \\
\hline$C(8)$ & $37(1)$ & $25(1)$ & $26(1)$ & $1(1)$ & $4(1)$ & $-1(1)$ \\
\hline$C(9)$ & $35(1)$ & $43(1)$ & $37(1)$ & $2(1)$ & $-7(1)$ & $-4(1)$ \\
\hline$C(10)$ & $28(1)$ & $42(1)$ & $30(1)$ & $6(1)$ & $3(1)$ & $3(1)$ \\
\hline$C(11)$ & $42(1)$ & $47(1)$ & $36(1)$ & $4(1)$ & 11(1) & $10(1)$ \\
\hline$C(12)$ & $55(1)$ & $74(1)$ & $43(1)$ & $9(1)$ & 19(1) & $24(1)$ \\
\hline$C(13)$ & $37(1)$ & $110(2)$ & $50(1)$ & $27(1)$ & 19(1) & $17(1)$ \\
\hline$C(14)$ & $38(1)$ & $98(2)$ & $58(1)$ & $21(1)$ & $10(1)$ & $-16(1)$ \\
\hline$C(15)$ & $42(1)$ & $65(1)$ & $45(1)$ & $5(1)$ & $7(1)$ & $-13(1)$ \\
\hline$C(16)$ & $27(1)$ & $32(1)$ & $22(1)$ & $-1(1)$ & $5(1)$ & $2(1)$ \\
\hline$C(17)$ & $28(1)$ & $34(1)$ & $26(1)$ & $0(1)$ & $7(1)$ & $1(1)$ \\
\hline $\mathrm{O}(1)$ & $46(1)$ & $56(1)$ & $26(1)$ & $7(1)$ & $-2(1)$ & $-11(1)$ \\
\hline $\mathrm{O}(2)$ & $45(1)$ & $34(1)$ & $32(1)$ & $6(1)$ & $2(1)$ & $-6(1)$ \\
\hline$C(18)$ & $50(1)$ & $40(1)$ & $41(1)$ & $14(1)$ & $9(1)$ & $1(1)$ \\
\hline $\mathrm{C}(19)$ & $64(1)$ & $67(1)$ & $65(1)$ & $18(1)$ & $33(1)$ & $-1(1)$ \\
\hline$C(20)$ & 124(2) & $44(1)$ & $74(1)$ & 11(1) & $21(1)$ & $-22(1)$ \\
\hline $\mathrm{C}(21)$ & $31(1)$ & $34(1)$ & $21(1)$ & $-1(1)$ & $3(1)$ & $2(1)$ \\
\hline
\end{tabular}




$\begin{array}{lcccccc}\mathrm{O}(3) & 44(1) & 32(1) & 24(1) & 0(1) & 2(1) & -5(1) \\ \mathrm{C}(22) & 41(1) & 32(1) & 27(1) & -1(1) & 6(1) & -6(1) \\ \mathrm{C}(23) & 41(1) & 32(1) & 32(1) & 1(1) & 5(1) & -4(1) \\ \mathrm{O}(4) & 47(1) & 54(1) & 70(1) & -15(1) & 22(1) & -12(1) \\ \mathrm{O}(5) & 56(1) & 30(1) & 62(1) & -8(1) & 26(1) & -6(1) \\ \mathrm{C}(24) & 73(1) & 34(1) & 72(1) & -5(1) & 41(1) & -1(1) \\ \mathrm{C}(25) & 108(2) & 58(1) & 109(2) & -40(1) & 52(2) & -22(1) \\ \mathrm{C}(26) & 106(2) & 58(1) & 108(2) & 20(1) & 55(2) & 31(1)\end{array}$

Table 5. Hydrogen coordinates ( $\left.x 10^{4}\right)$ and isotropic displacement parameters $\left(\AA^{2} \mathrm{x} 10^{3}\right)$ for Sana-A.

\begin{tabular}{|c|c|c|c|c|}
\hline & $\mathrm{x}$ & $\mathrm{y}$ & $\mathrm{z}$ & $\mathrm{U}(\mathrm{eq})$ \\
\hline $\mathrm{H}(4)$ & 277 & 5607 & 3802 & 41 \\
\hline $\mathrm{H}(5)$ & 154 & 5678 & 5616 & 50 \\
\hline $\mathrm{H}(6)$ & 2355 & 5793 & 6943 & 52 \\
\hline $\mathrm{H}(7)$ & 4737 & 5862 & 6497 & 45 \\
\hline $\mathrm{H}(9 \mathrm{~A})$ & 7047 & 6244 & 5130 & 61 \\
\hline $\mathrm{H}(9 \mathrm{~B})$ & 7367 & 5627 & 4546 & 61 \\
\hline $\mathrm{H}(9 \mathrm{C})$ & 6795 & 5598 & 5650 & 61 \\
\hline $\mathrm{H}(11)$ & 4967 & 4968 & 1710 & 50 \\
\hline $\mathrm{H}(12)$ & 6624 & 5004 & 519 & 67 \\
\hline $\mathrm{H}(13)$ & 8392 & 5790 & 635 & 77 \\
\hline $\mathrm{H}(14)$ & 8486 & 6557 & 1919 & 78 \\
\hline $\mathrm{H}(15)$ & 6801 & 6544 & 3085 & 61 \\
\hline $\mathrm{H}(18)$ & 1812 & 6912 & -100 & 52 \\
\hline $\mathrm{H}(19 \mathrm{~A})$ & 4792 & 6441 & -33 & 94 \\
\hline $\mathrm{H}(19 \mathrm{~B})$ & 3856 & 6824 & -1018 & 94 \\
\hline $\mathrm{H}(19 \mathrm{C})$ & 3272 & 6165 & -745 & 94 \\
\hline $\mathrm{H}(20 \mathrm{~A})$ & 2936 & 7592 & 1266 & 121 \\
\hline $\mathrm{H}(20 \mathrm{~B})$ & 3611 & 7719 & 224 & 121 \\
\hline $\mathrm{H}(20 \mathrm{C})$ & 4580 & 7345 & 1208 & 121 \\
\hline
\end{tabular}




\begin{tabular}{lrrrr}
$\mathrm{H}(21)$ & 660 & 4929 & 1124 & 35 \\
$\mathrm{H}(22 \mathrm{~A})$ & -188 & 4026 & 1694 & 40 \\
$\mathrm{H}(22 \mathrm{~B})$ & 549 & 3745 & 2843 & 40 \\
$\mathrm{H}(24)$ & 2953 & 3008 & 440 & 67 \\
$\mathrm{H}(25 \mathrm{~A})$ & 636 & 2107 & 345 & 131 \\
$\mathrm{H}(25 \mathrm{~B})$ & 1810 & 2121 & -435 & 131 \\
$\mathrm{H}(25 \mathrm{C})$ & 585 & 2654 & -493 & 131 \\
$\mathrm{H}(26 \mathrm{~A})$ & 4063 & 2614 & 2142 & 129 \\
$\mathrm{H}(26 \mathrm{~B})$ & 4036 & 2093 & 1258 & 129 \\
$\mathrm{H}(26 \mathrm{C})$ & 2822 & 2083 & 2008 & 129 \\
& & & & \\
\hline
\end{tabular}

Table 6. Torsion angles $\left[{ }^{\circ}\right]$ for Sana-A.

\begin{tabular}{lc}
\hline $\mathrm{C}(8)-\mathrm{N}(1)-\mathrm{C}(1)-\mathrm{C}(2)$ & $0.85(15)$ \\
$\mathrm{C}(9)-\mathrm{N}(1)-\mathrm{C}(1)-\mathrm{C}(2)$ & $170.21(13)$ \\
$\mathrm{C}(8)-\mathrm{N}(1)-\mathrm{C}(1)-\mathrm{C}(10)$ & $179.79(12)$ \\
$\mathrm{C}(9)-\mathrm{N}(1)-\mathrm{C}(1)-\mathrm{C}(10)$ & $-10.9(2)$ \\
$\mathrm{N}(1)-\mathrm{C}(1)-\mathrm{C}(2)-\mathrm{C}(3)$ & $0.18(15)$ \\
$\mathrm{C}(10)-\mathrm{C}(1)-\mathrm{C}(2)-\mathrm{C}(3)$ & $-178.65(13)$ \\
$\mathrm{N}(1)-\mathrm{C}(1)-\mathrm{C}(2)-\mathrm{C}(16)$ & $-176.16(12)$ \\
$\mathrm{C}(10)-\mathrm{C}(1)-\mathrm{C}(2)-\mathrm{C}(16)$ & $5.0(2)$ \\
$\mathrm{C}(1)-\mathrm{C}(2)-\mathrm{C}(3)-\mathrm{C}(4)$ & $178.74(15)$ \\
$\mathrm{C}(16)-\mathrm{C}(2)-\mathrm{C}(3)-\mathrm{C}(4)$ & $-4.8(2)$ \\
$\mathrm{C}(1)-\mathrm{C}(2)-\mathrm{C}(3)-\mathrm{C}(8)$ & $-1.11(14)$ \\
$\mathrm{C}(16)-\mathrm{C}(2)-\mathrm{C}(3)-\mathrm{C}(8)$ & $175.36(12)$ \\
$\mathrm{C}(8)-\mathrm{C}(3)-\mathrm{C}(4)-\mathrm{C}(5)$ & $-1.4(2)$ \\
$\mathrm{C}(2)-\mathrm{C}(3)-\mathrm{C}(4)-\mathrm{C}(5)$ & $178.82(14)$ \\
$\mathrm{C}(3)-\mathrm{C}(4)-\mathrm{C}(5)-\mathrm{C}(6)$ & $-0.2(2)$ \\
$\mathrm{C}(4)-\mathrm{C}(5)-\mathrm{C}(6)-\mathrm{C}(7)$ & $0.9(3)$ \\
$\mathrm{C}(5)-\mathrm{C}(6)-\mathrm{C}(7)-\mathrm{C}(8)$ & $0.0(2)$ \\
$\mathrm{C}(1)-\mathrm{N}(1)-\mathrm{C}(8)-\mathrm{C}(7)$ & $177.84(14)$ \\
$\mathrm{C}(9)-\mathrm{N}(1)-\mathrm{C}(8)-\mathrm{C}(7)$ & $8.1(2)$ \\
$\mathrm{C}(1)-\mathrm{N}(1)-\mathrm{C}(8)-\mathrm{C}(3)$ & $-1.55(15)$ \\
$\mathrm{C}(9)-\mathrm{N}(1)-\mathrm{C}(8)-\mathrm{C}(3)$ & $-171.32(12)$ \\
& \\
& \\
&
\end{tabular}




\begin{tabular}{|c|c|}
\hline $\mathrm{C}(6)-\mathrm{C}(7)-\mathrm{C}(8)-\mathrm{N}(1)$ & $179.09(14)$ \\
\hline $\mathrm{C}(6)-\mathrm{C}(7)-\mathrm{C}(8)-\mathrm{C}(3)$ & $-1.6(2)$ \\
\hline $\mathrm{C}(4)-\mathrm{C}(3)-\mathrm{C}(8)-\mathrm{N}(1)$ & $-178.24(12)$ \\
\hline $\mathrm{C}(2)-\mathrm{C}(3)-\mathrm{C}(8)-\mathrm{N}(1)$ & $1.64(14)$ \\
\hline$C(4)-C(3)-C(8)-C(7)$ & $2.3(2)$ \\
\hline $\mathrm{C}(2)-\mathrm{C}(3)-\mathrm{C}(8)-\mathrm{C}(7)$ & $-177.82(12)$ \\
\hline$C(2)-C(1)-C(10)-C(15)$ & $129.55(16)$ \\
\hline $\mathrm{N}(1)-\mathrm{C}(1)-\mathrm{C}(10)-\mathrm{C}(15)$ & $-49.2(2)$ \\
\hline $\mathrm{C}(2)-\mathrm{C}(1)-\mathrm{C}(10)-\mathrm{C}(11)$ & $-49.3(2)$ \\
\hline $\mathrm{N}(1)-\mathrm{C}(1)-\mathrm{C}(10)-\mathrm{C}(11)$ & 132.01(14) \\
\hline $\mathrm{C}(15)-\mathrm{C}(10)-\mathrm{C}(11)-\mathrm{C}(12)$ & $0.3(2)$ \\
\hline $\mathrm{C}(1)-\mathrm{C}(10)-\mathrm{C}(11)-\mathrm{C}(12)$ & $179.18(14)$ \\
\hline$C(10)-C(11)-C(12)-C(13)$ & $0.7(2)$ \\
\hline $\mathrm{C}(11)-\mathrm{C}(12)-\mathrm{C}(13)-\mathrm{C}(14)$ & $-0.8(3)$ \\
\hline $\mathrm{C}(12)-\mathrm{C}(13)-\mathrm{C}(14)-\mathrm{C}(15)$ & $-0.1(3)$ \\
\hline $\mathrm{C}(13)-\mathrm{C}(14)-\mathrm{C}(15)-\mathrm{C}(10)$ & $1.1(3)$ \\
\hline$C(11)-C(10)-C(15)-C(14)$ & $-1.2(2)$ \\
\hline $\mathrm{C}(1)-\mathrm{C}(10)-\mathrm{C}(15)-\mathrm{C}(14)$ & $179.94(15)$ \\
\hline$C(1)-C(2)-C(16)-C(21)$ & $118.31(16)$ \\
\hline $\mathrm{C}(3)-\mathrm{C}(2)-\mathrm{C}(16)-\mathrm{C}(21)$ & $-57.42(19)$ \\
\hline $\mathrm{C}(1)-\mathrm{C}(2)-\mathrm{C}(16)-\mathrm{C}(17)$ & $-60.9(2)$ \\
\hline $\mathrm{C}(3)-\mathrm{C}(2)-\mathrm{C}(16)-\mathrm{C}(17)$ & $123.32(15)$ \\
\hline $\mathrm{C}(21)-\mathrm{C}(16)-\mathrm{C}(17)-\mathrm{O}(1)$ & $2.7(2)$ \\
\hline $\mathrm{C}(2)-\mathrm{C}(16)-\mathrm{C}(17)-\mathrm{O}(1)$ & $-178.02(13)$ \\
\hline $\mathrm{C}(21)-\mathrm{C}(16)-\mathrm{C}(17)-\mathrm{O}(2)$ & $-176.76(12)$ \\
\hline $\mathrm{C}(2)-\mathrm{C}(16)-\mathrm{C}(17)-\mathrm{O}(2)$ & $2.54(18)$ \\
\hline $\mathrm{O}(1)-\mathrm{C}(17)-\mathrm{O}(2)-\mathrm{C}(18)$ & $-5.0(2)$ \\
\hline $\mathrm{C}(16)-\mathrm{C}(17)-\mathrm{O}(2)-\mathrm{C}(18)$ & $174.43(12)$ \\
\hline $\mathrm{C}(17)-\mathrm{O}(2)-\mathrm{C}(18)-\mathrm{C}(20)$ & $163.65(15)$ \\
\hline $\mathrm{C}(17)-\mathrm{O}(2)-\mathrm{C}(18)-\mathrm{C}(19)$ & $-74.62(18)$ \\
\hline $\mathrm{C}(2)-\mathrm{C}(16)-\mathrm{C}(21)-\mathrm{O}(3)$ & $0.9(2)$ \\
\hline $\mathrm{C}(17)-\mathrm{C}(16)-\mathrm{C}(21)-\mathrm{O}(3)$ & $-179.74(12)$ \\
\hline $\mathrm{C}(16)-\mathrm{C}(21)-\mathrm{O}(3)-\mathrm{C}(22)$ & $-161.48(13)$ \\
\hline $\mathrm{C}(21)-\mathrm{O}(3)-\mathrm{C}(22)-\mathrm{C}(23)$ & $74.65(14)$ \\
\hline $\mathrm{O}(3)-\mathrm{C}(22)-\mathrm{C}(23)-\mathrm{O}(4)$ & $0.8(2)$ \\
\hline
\end{tabular}


$\mathrm{O}(3)-\mathrm{C}(22)-\mathrm{C}(23)-\mathrm{O}(5)$

$\mathrm{O}(4)-\mathrm{C}(23)-\mathrm{O}(5)-\mathrm{C}(24)$

$\mathrm{C}(22)-\mathrm{C}(23)-\mathrm{O}(5)-\mathrm{C}(24)$

$\mathrm{C}(23)-\mathrm{O}(5)-\mathrm{C}(24)-\mathrm{C}(25)$

$\mathrm{C}(23)-\mathrm{O}(5)-\mathrm{C}(24)-\mathrm{C}(26)$
$-178.20(11)$

$-2.5(2)$

$176.53(13)$

$-148.15(16)$

$88.27(19)$

Symmetry transformations used to generate equivalent atoms: 


\section{Crystal refinement data for compound 7c}

Empirical Formula- $\mathrm{C}_{15} \mathrm{H}_{17} \mathrm{NO}_{3}, \mathrm{M}=259.29$, Monoclinic, Space group $\mathrm{P} 2{ }_{1} / \mathrm{c}, \mathrm{a}=7.175(2) \AA, \mathrm{b}=$ 18.551(4) $\AA, c=10.399(2) \AA, V=1350.9(5) \AA^{3}, Z=4, T=223(2) \mathrm{K}, \rho c a l c d=1.275 \mathrm{Mg} / \mathrm{m}^{3}, 2 \Theta_{\text {max. }}=$ $28.260^{\circ}$, Refinement of 552 parameters on 3341 independent reflections out of 22289 collected reflections $\left(\mathrm{R}_{\text {int }}=0.0321\right)$ led to $\mathrm{R} 1=0.0458[\mathrm{I}>2 \sigma(\mathrm{I})]$, wR $2=0.1252$ (all data) and $\mathrm{S}=1.041$ with the largest difference peak and hole of 0.260 and -0.211 e. $\AA^{-3}$ respectively. The crystal structure has been deposited at the Cambridge Crystallographic Data Centre (CCDC 2057965). The data can be obtained free of charge via the Internet at www.ccdc.cam.ac.uk/data_request/cif

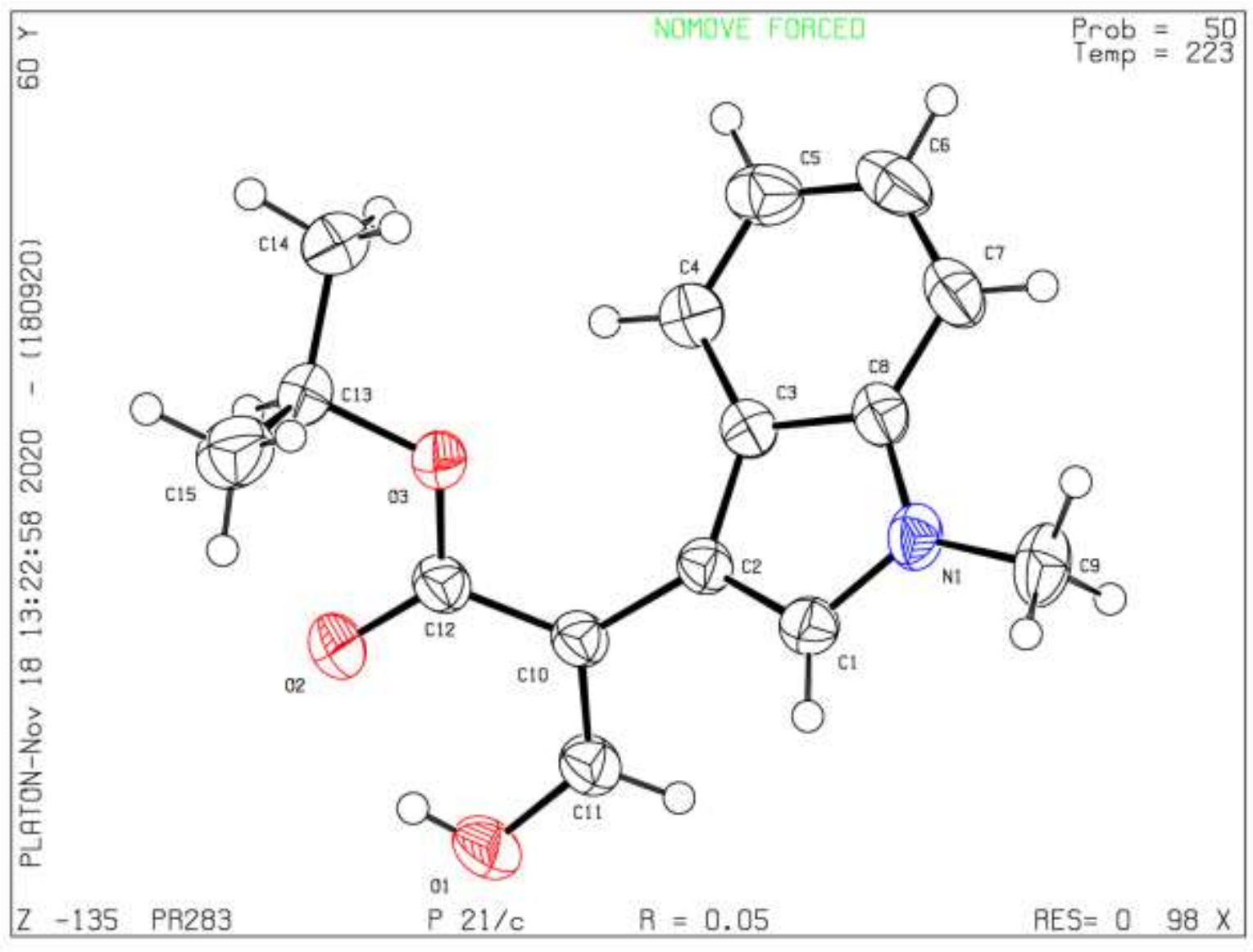

Figure S3. X-ray structure of compound 7c (ellipsoid contour \% probability-50). 
Table 1. Crystal data and structure refinement for 7c (PR283).

Identification code

Empirical formula

Formula weight

Temperature

Wavelength

Crystal system

Space group

Unit cell dimensions

Volume

Z

Density (calculated)

Absorption coefficient

$\mathrm{F}(000)$

Crystal size

Theta range for data collection

Index ranges

Reflections collected

Independent reflections

Completeness to theta $=25.242^{\circ}$

Absorption correction

Max. and min. transmission

Refinement method

Data / restraints / parameters

Goodness-of-fit on $\mathrm{F}^{2}$

Final R indices [I $>2 \operatorname{sigma}(\mathrm{I})]$

$\mathrm{R}$ indices (all data)

Extinction coefficient

Largest diff. peak and hole
PR283

C15 H17 N O3

259.29

223(2) K

$0.71073 \AA$

Monoclinic

$\mathrm{P} 2{ }_{1} / \mathrm{c}$

$\mathrm{a}=7.175(2) \AA$

$\alpha=90^{\circ}$.

$\mathrm{b}=18.551(4) \AA$

$\beta=102.592(8)^{\circ}$.

$\mathrm{c}=10.399(2) \AA$

$\gamma=90^{\circ}$.
4

$1.275 \mathrm{Mg} / \mathrm{m}^{3}$

$0.089 \mathrm{~mm}^{-1}$

552

$0.422 \times 0.272 \times 0.215 \mathrm{~mm}^{3}$

2.287 to $28.260^{\circ}$.

$-9<=\mathrm{h}<=9,-24<=\mathrm{k}<=24,-13<=1<=13$

22289

$3341[\mathrm{R}(\mathrm{int})=0.0321]$

$99.8 \%$

Semi-empirical from equivalents

0.7457 and 0.6800

Full-matrix least-squares on $\mathrm{F}^{2}$

3341 / 0 / 176

1.041

$\mathrm{R} 1=0.0458, w \mathrm{R} 2=0.1159$

$\mathrm{R} 1=0.0608, \mathrm{wR} 2=0.1252$

$\mathrm{n} / \mathrm{a}$

0.260 and $-0.211 \mathrm{e} . \AA^{-3}$ 
Table 2. Atomic coordinates ( $\mathrm{x} 10^{4}$ ) and equivalent isotropic displacement parameters $\left(\AA^{2} \times 10^{3}\right)$ for PR283. U(eq) is defined as one third of the trace of the orthogonalized $U^{\mathrm{ij}}$ tensor.

\begin{tabular}{|c|c|c|c|c|}
\hline & $\mathrm{x}$ & $\mathrm{y}$ & $\mathrm{z}$ & $\mathrm{U}(\mathrm{eq})$ \\
\hline $\mathrm{N}(1)$ & $2639(2)$ & $4750(1)$ & $5322(1)$ & $38(1)$ \\
\hline $\mathrm{C}(1)$ & $2946(2)$ & $4752(1)$ & $6665(1)$ & $36(1)$ \\
\hline $\mathrm{C}(2)$ & $3020(2)$ & $5442(1)$ & $7142(1)$ & $31(1)$ \\
\hline $\mathrm{C}(3)$ & $2763(2)$ & $5907(1)$ & $6012(1)$ & $31(1)$ \\
\hline $\mathrm{C}(4)$ & $2708(2)$ & $6658(1)$ & $5812(2)$ & $39(1)$ \\
\hline $\mathrm{C}(5)$ & $2469(2)$ & $6919(1)$ & $4546(2)$ & $48(1)$ \\
\hline$C(6)$ & $2259(2)$ & $6457(1)$ & $3463(2)$ & $52(1)$ \\
\hline$C(7)$ & $2271(2)$ & $5722(1)$ & $3618(2)$ & $45(1)$ \\
\hline $\mathrm{C}(8)$ & $2530(2)$ & $5454(1)$ & $4896(1)$ & $35(1)$ \\
\hline$C(9)$ & $2487(2)$ & $4115(1)$ & $4487(2)$ & $50(1)$ \\
\hline$C(10)$ & $3219(2)$ & $5620(1)$ & $8548(1)$ & $33(1)$ \\
\hline$C(11)$ & $2145(2)$ & $5265(1)$ & $9258(1)$ & $39(1)$ \\
\hline $\mathrm{O}(1)$ & $2266(2)$ & $5324(1)$ & 10551(1) & $52(1)$ \\
\hline$C(12)$ & $4605(2)$ & $6144(1)$ & $9244(1)$ & $35(1)$ \\
\hline $\mathrm{O}(2)$ & $4795(2)$ & $6281(1)$ & $10424(1)$ & $48(1)$ \\
\hline $\mathrm{O}(3)$ & $5622(2)$ & $6469(1)$ & $8487(1)$ & $38(1)$ \\
\hline$C(13)$ & $6987(2)$ & $7027(1)$ & $9093(2)$ & $40(1)$ \\
\hline$C(14)$ & $7333(3)$ & $7476(1)$ & $7961(2)$ & $54(1)$ \\
\hline$C(15)$ & $8749(3)$ & $6686(1)$ & $9885(2)$ & $62(1)$ \\
\hline
\end{tabular}

Table 3. Bond lengths $[\AA]$ and angles $\left[{ }^{\circ}\right]$ for PR283.

\begin{tabular}{ll}
\hline $\mathrm{N}(1)-\mathrm{C}(1)$ & $1.3658(19)$ \\
$\mathrm{N}(1)-\mathrm{C}(8)$ & $1.374(2)$ \\
$\mathrm{N}(1)-\mathrm{C}(9)$ & $1.4532(19)$ \\
$\mathrm{C}(1)-\mathrm{C}(2)$ & $1.370(2)$ \\
$\mathrm{C}(1)-\mathrm{H}(1)$ & 0.9400 \\
$\mathrm{C}(2)-\mathrm{C}(3)$ & $1.4364(19)$ \\
$\mathrm{C}(2)-\mathrm{C}(10)$ & $1.4746(18)$
\end{tabular}




\begin{tabular}{|c|c|}
\hline $\mathrm{C}(3)-\mathrm{C}(4)$ & $1.407(2)$ \\
\hline $\mathrm{C}(3)-\mathrm{C}(8)$ & $1.4144(19)$ \\
\hline$C(4)-C(5)$ & $1.379(2)$ \\
\hline $\mathrm{C}(4)-\mathrm{H}(4)$ & 0.9400 \\
\hline$C(5)-C(6)$ & $1.398(3)$ \\
\hline $\mathrm{C}(5)-\mathrm{H}(5)$ & 0.9400 \\
\hline$C(6)-C(7)$ & $1.373(3)$ \\
\hline $\mathrm{C}(6)-\mathrm{H}(6)$ & 0.9400 \\
\hline$C(7)-C(8)$ & $1.392(2)$ \\
\hline $\mathrm{C}(7)-\mathrm{H}(7)$ & 0.9400 \\
\hline $\mathrm{C}(9)-\mathrm{H}(9 \mathrm{~A})$ & 0.9700 \\
\hline C(9)-H(9B) & 0.9700 \\
\hline C(9)-H(9C) & 0.9700 \\
\hline $\mathrm{C}(10)-\mathrm{C}(11)$ & $1.350(2)$ \\
\hline $\mathrm{C}(10)-\mathrm{C}(12)$ & $1.464(2)$ \\
\hline $\mathrm{C}(11)-\mathrm{O}(1)$ & $1.3335(18)$ \\
\hline $\mathrm{C}(11)-\mathrm{H}(11)$ & 0.9400 \\
\hline $\mathrm{O}(1)-\mathrm{H}(1 \mathrm{~A})$ & 0.8300 \\
\hline $\mathrm{C}(12)-\mathrm{O}(2)$ & $1.2320(17)$ \\
\hline $\mathrm{C}(12)-\mathrm{O}(3)$ & $1.3292(17)$ \\
\hline $\mathrm{O}(3)-\mathrm{C}(13)$ & $1.4689(17)$ \\
\hline $\mathrm{C}(13)-\mathrm{C}(15)$ & $1.490(2)$ \\
\hline $\mathrm{C}(13)-\mathrm{C}(14)$ & $1.507(2)$ \\
\hline C(13)-H(13) & 0.9900 \\
\hline $\mathrm{C}(14)-\mathrm{H}(14 \mathrm{~A})$ & 0.9700 \\
\hline $\mathrm{C}(14)-\mathrm{H}(14 \mathrm{~B})$ & 0.9700 \\
\hline $\mathrm{C}(14)-\mathrm{H}(14 \mathrm{C})$ & 0.9700 \\
\hline $\mathrm{C}(15)-\mathrm{H}(15 \mathrm{~A})$ & 0.9700 \\
\hline $\mathrm{C}(15)-\mathrm{H}(15 \mathrm{~B})$ & 0.9700 \\
\hline $\mathrm{C}(15)-\mathrm{H}(15 \mathrm{C})$ & 0.9700 \\
\hline $\mathrm{C}(1)-\mathrm{N}(1)-\mathrm{C}(8)$ & $108.25(11)$ \\
\hline $\mathrm{C}(1)-\mathrm{N}(1)-\mathrm{C}(9)$ & $125.92(14)$ \\
\hline $\mathrm{C}(8)-\mathrm{N}(1)-\mathrm{C}(9)$ & $125.82(13)$ \\
\hline $\mathrm{N}(1)-\mathrm{C}(1)-\mathrm{C}(2)$ & $110.90(13)$ \\
\hline
\end{tabular}




\begin{tabular}{|c|c|}
\hline $\mathrm{N}(1)-\mathrm{C}(1)-\mathrm{H}(1)$ & 124.5 \\
\hline $\mathrm{C}(2)-\mathrm{C}(1)-\mathrm{H}(1)$ & 124.5 \\
\hline$C(1)-C(2)-C(3)$ & $106.10(12)$ \\
\hline$C(1)-C(2)-C(10)$ & $123.73(13)$ \\
\hline$C(3)-C(2)-C(10)$ & $130.08(13)$ \\
\hline$C(4)-C(3)-C(8)$ & $118.23(13)$ \\
\hline$C(4)-C(3)-C(2)$ & $135.21(13)$ \\
\hline $\mathrm{C}(8)-\mathrm{C}(3)-\mathrm{C}(2)$ & $106.57(12)$ \\
\hline$C(5)-C(4)-C(3)$ & $118.90(15)$ \\
\hline $\mathrm{C}(5)-\mathrm{C}(4)-\mathrm{H}(4)$ & 120.5 \\
\hline $\mathrm{C}(3)-\mathrm{C}(4)-\mathrm{H}(4)$ & 120.5 \\
\hline$C(4)-C(5)-C(6)$ & $121.52(16)$ \\
\hline $\mathrm{C}(4)-\mathrm{C}(5)-\mathrm{H}(5)$ & 119.2 \\
\hline $\mathrm{C}(6)-\mathrm{C}(5)-\mathrm{H}(5)$ & 119.2 \\
\hline$C(7)-C(6)-C(5)$ & $121.18(15)$ \\
\hline $\mathrm{C}(7)-\mathrm{C}(6)-\mathrm{H}(6)$ & 119.4 \\
\hline $\mathrm{C}(5)-\mathrm{C}(6)-\mathrm{H}(6)$ & 119.4 \\
\hline$C(6)-C(7)-C(8)$ & $117.62(15)$ \\
\hline $\mathrm{C}(6)-\mathrm{C}(7)-\mathrm{H}(7)$ & 121.2 \\
\hline $\mathrm{C}(8)-\mathrm{C}(7)-\mathrm{H}(7)$ & 121.2 \\
\hline $\mathrm{N}(1)-\mathrm{C}(8)-\mathrm{C}(7)$ & $129.28(14)$ \\
\hline $\mathrm{N}(1)-\mathrm{C}(8)-\mathrm{C}(3)$ & $108.18(12)$ \\
\hline $\mathrm{C}(7)-\mathrm{C}(8)-\mathrm{C}(3)$ & $122.53(15)$ \\
\hline $\mathrm{N}(1)-\mathrm{C}(9)-\mathrm{H}(9 \mathrm{~A})$ & 109.5 \\
\hline $\mathrm{N}(1)-\mathrm{C}(9)-\mathrm{H}(9 \mathrm{~B})$ & 109.5 \\
\hline $\mathrm{H}(9 \mathrm{~A})-\mathrm{C}(9)-\mathrm{H}(9 \mathrm{~B})$ & 109.5 \\
\hline $\mathrm{N}(1)-\mathrm{C}(9)-\mathrm{H}(9 \mathrm{C})$ & 109.5 \\
\hline $\mathrm{H}(9 \mathrm{~A})-\mathrm{C}(9)-\mathrm{H}(9 \mathrm{C})$ & 109.5 \\
\hline $\mathrm{H}(9 \mathrm{~B})-\mathrm{C}(9)-\mathrm{H}(9 \mathrm{C})$ & 109.5 \\
\hline $\mathrm{C}(11)-\mathrm{C}(10)-\mathrm{C}(12)$ & $117.43(13)$ \\
\hline $\mathrm{C}(11)-\mathrm{C}(10)-\mathrm{C}(2)$ & $119.31(13)$ \\
\hline $\mathrm{C}(12)-\mathrm{C}(10)-\mathrm{C}(2)$ & $123.18(12)$ \\
\hline $\mathrm{O}(1)-\mathrm{C}(11)-\mathrm{C}(10)$ & $126.31(15)$ \\
\hline $\mathrm{O}(1)-\mathrm{C}(11)-\mathrm{H}(11)$ & 116.8 \\
\hline $\mathrm{C}(10)-\mathrm{C}(11)-\mathrm{H}(11)$ & 116.8 \\
\hline
\end{tabular}




$\begin{array}{ll}\mathrm{C}(11)-\mathrm{O}(1)-\mathrm{H}(1 \mathrm{~A}) & 109.5 \\ \mathrm{O}(2)-\mathrm{C}(12)-\mathrm{O}(3) & 122.71(13) \\ \mathrm{O}(2)-\mathrm{C}(12)-\mathrm{C}(10) & 123.10(13) \\ \mathrm{O}(3)-\mathrm{C}(12)-\mathrm{C}(10) & 114.18(12) \\ \mathrm{C}(12)-\mathrm{O}(3)-\mathrm{C}(13) & 117.79(11) \\ \mathrm{O}(3)-\mathrm{C}(13)-\mathrm{C}(15) & 110.17(13) \\ \mathrm{O}(3)-\mathrm{C}(13)-\mathrm{C}(14) & 105.20(12) \\ \mathrm{C}(15)-\mathrm{C}(13)-\mathrm{C}(14) & 113.71(16) \\ \mathrm{O}(3)-\mathrm{C}(13)-\mathrm{H}(13) & 109.2 \\ \mathrm{C}(15)-\mathrm{C}(13)-\mathrm{H}(13) & 109.2 \\ \mathrm{C}(14)-\mathrm{C}(13)-\mathrm{H}(13) & 109.2 \\ \mathrm{C}(13)-\mathrm{C}(14)-\mathrm{H}(14 \mathrm{~A}) & 109.5 \\ \mathrm{C}(13)-\mathrm{C}(14)-\mathrm{H}(14 \mathrm{~B}) & 109.5 \\ \mathrm{H}(14 \mathrm{~A})-\mathrm{C}(14)-\mathrm{H}(14 \mathrm{~B}) & 109.5 \\ \mathrm{C}(13)-\mathrm{C}(14)-\mathrm{H}(14 \mathrm{C}) & 109.5 \\ \mathrm{H}(14 \mathrm{~A})-\mathrm{C}(14)-\mathrm{H}(14 \mathrm{C}) & 109.5 \\ \mathrm{H}(14 \mathrm{~B})-\mathrm{C}(14)-\mathrm{H}(14 \mathrm{C}) & 109.5 \\ \mathrm{C}(13)-\mathrm{C}(15)-\mathrm{H}(15 \mathrm{~A}) & 109.5 \\ \mathrm{C}(13)-\mathrm{C}(15)-\mathrm{H}(15 \mathrm{~B}) & 109.5 \\ \mathrm{H}(15 \mathrm{~A})-\mathrm{C}(15)-\mathrm{H}(15 \mathrm{~B}) & 109.5 \\ \mathrm{C}(13)-\mathrm{C}(15)-\mathrm{H}(15 \mathrm{C}) & 109.5 \\ \mathrm{H}(15 \mathrm{~A})-\mathrm{C}(15)-\mathrm{H}(15 \mathrm{C}) & 109.5 \\ \mathrm{H}(15 \mathrm{~B})-\mathrm{C}(15)-\mathrm{H}(15 \mathrm{C}) & 109.5 \\ & \end{array}$

Symmetry transformations used to generate equivalent atoms:

Table 4. Anisotropic displacement parameters $\left(\AA^{2} \times 10^{3}\right)$ for PR283. The anisotropic displacement factor exponent takes the form: $-2 \pi^{2}\left[h^{2} a^{* 2} U^{11}+\ldots+2 h k a^{*} b^{*} U^{12}\right]$

\begin{tabular}{lllllll}
\hline & $\mathrm{U}^{11}$ & $\mathrm{U}^{22}$ & $\mathrm{U}^{33}$ & $\mathrm{U}^{23}$ & $\mathrm{U}^{13}$ & $\mathrm{U}^{12}$ \\
\hline $\mathrm{N}(1)$ & $40(1)$ & $40(1)$ & $36(1)$ & $-10(1)$ & $11(1)$ & $-4(1)$ \\
$\mathrm{C}(1)$ & $38(1)$ & $36(1)$ & $35(1)$ & $-1(1)$ & $11(1)$ & $-3(1)$ \\
$\mathrm{C}(2)$ & $32(1)$ & $34(1)$ & $29(1)$ & $-2(1)$ & $9(1)$ & $-1(1)$ \\
$\mathrm{C}(3)$ & $26(1)$ & $38(1)$ & $30(1)$ & $-1(1)$ & $7(1)$ & $1(1)$ \\
& & & & & &
\end{tabular}




\begin{tabular}{lllllll}
$\mathrm{C}(4)$ & $34(1)$ & $40(1)$ & $42(1)$ & $2(1)$ & $7(1)$ & $3(1)$ \\
$\mathrm{C}(5)$ & $42(1)$ & $49(1)$ & $52(1)$ & $16(1)$ & $7(1)$ & $4(1)$ \\
$\mathrm{C}(6)$ & $47(1)$ & $73(1)$ & $35(1)$ & $15(1)$ & $6(1)$ & $3(1)$ \\
$\mathrm{C}(7)$ & $40(1)$ & $66(1)$ & $29(1)$ & $-2(1)$ & $7(1)$ & $-3(1)$ \\
$\mathrm{C}(8)$ & $27(1)$ & $46(1)$ & $31(1)$ & $-4(1)$ & $7(1)$ & $-2(1)$ \\
$\mathrm{C}(9)$ & $47(1)$ & $52(1)$ & $52(1)$ & $-24(1)$ & $14(1)$ & $-7(1)$ \\
$\mathrm{C}(10)$ & $37(1)$ & $34(1)$ & $29(1)$ & $0(1)$ & $9(1)$ & $1(1)$ \\
$\mathrm{C}(11)$ & $41(1)$ & $45(1)$ & $33(1)$ & $-1(1)$ & $12(1)$ & $-3(1)$ \\
$\mathrm{O}(1)$ & $60(1)$ & $67(1)$ & $32(1)$ & $1(1)$ & $19(1)$ & $-12(1)$ \\
$\mathrm{C}(12)$ & $42(1)$ & $35(1)$ & $29(1)$ & $-1(1)$ & $10(1)$ & $1(1)$ \\
$\mathrm{O}(2)$ & $65(1)$ & $52(1)$ & $29(1)$ & $-8(1)$ & $13(1)$ & $-13(1)$ \\
$\mathrm{O}(3)$ & $45(1)$ & $39(1)$ & $31(1)$ & $-4(1)$ & $10(1)$ & $-11(1)$ \\
$\mathrm{C}(13)$ & $46(1)$ & $34(1)$ & $38(1)$ & $-4(1)$ & $5(1)$ & $-7(1)$ \\
$\mathrm{C}(14)$ & $62(1)$ & $50(1)$ & $48(1)$ & $5(1)$ & $6(1)$ & $-17(1)$ \\
$\mathrm{C}(15)$ & $51(1)$ & $56(1)$ & $70(1)$ & $13(1)$ & $-3(1)$ & $-8(1)$ \\
& & & & & & \\
\hline
\end{tabular}

Table 5. Hydrogen coordinates ( $\left.\mathrm{x} 10^{4}\right)$ and isotropic displacement parameters $\left(\AA^{2} \mathrm{x} 10^{3}\right)$ for PR283.

\begin{tabular}{lrrrl}
\hline & $\mathrm{x}$ & $\mathrm{y}$ & $\mathrm{z}$ & $\mathrm{U}(\mathrm{eq})$ \\
& & & & \\
$\mathrm{H}(1)$ & 3087 & 4335 & 7192 & 43 \\
$\mathrm{H}(4)$ & 2832 & 6975 & 6530 & 47 \\
$\mathrm{H}(5)$ & 2446 & 7420 & 4408 & 58 \\
$\mathrm{H}(6)$ & 2108 & 6652 & 2612 & 63 \\
$\mathrm{H}(7)$ & 2109 & 5411 & 2889 & 54 \\
$\mathrm{H}(9 \mathrm{~A})$ & 3223 & 3725 & 4972 & 75 \\
$\mathrm{H}(9 \mathrm{~B})$ & 2979 & 4225 & 3711 & 75 \\
$\mathrm{H}(9 \mathrm{C})$ & 1158 & 3972 & 4222 & 75 \\
$\mathrm{H}(11)$ & 1222 & 4945 & 8795 & 47 \\
$\mathrm{H}(1 \mathrm{~A})$ & 3089 & 5629 & 10862 & 77 \\
$\mathrm{H}(13)$ & 6395 & 7331 & 9676 & 48 \\
$\mathrm{H}(14 \mathrm{~A})$ & 7855 & 7174 & 7363 & 81 \\
& & & &
\end{tabular}




\begin{tabular}{lrrrr}
$\mathrm{H}(14 \mathrm{~B})$ & 8231 & 7858 & 8296 & 81 \\
$\mathrm{H}(14 \mathrm{C})$ & 6137 & 7685 & 7494 & 81 \\
$\mathrm{H}(15 \mathrm{~A})$ & 8418 & 6389 & 10570 & 92 \\
$\mathrm{H}(15 \mathrm{~B})$ & 9636 & 7059 & 10285 & 92 \\
$\mathrm{H}(15 \mathrm{C})$ & 9338 & 6389 & 9316 & 92 \\
\hline
\end{tabular}

Table 6. Torsion angles $\left[{ }^{\circ}\right]$ for PR283.

\begin{tabular}{lc}
\hline $\mathrm{C}(8)-\mathrm{N}(1)-\mathrm{C}(1)-\mathrm{C}(2)$ & $0.76(17)$ \\
$\mathrm{C}(9)-\mathrm{N}(1)-\mathrm{C}(1)-\mathrm{C}(2)$ & $179.78(14)$ \\
$\mathrm{N}(1)-\mathrm{C}(1)-\mathrm{C}(2)-\mathrm{C}(3)$ & $-0.74(16)$ \\
$\mathrm{N}(1)-\mathrm{C}(1)-\mathrm{C}(2)-\mathrm{C}(10)$ & $176.23(12)$ \\
$\mathrm{C}(1)-\mathrm{C}(2)-\mathrm{C}(3)-\mathrm{C}(4)$ & $-179.34(16)$ \\
$\mathrm{C}(10)-\mathrm{C}(2)-\mathrm{C}(3)-\mathrm{C}(4)$ & $4.0(3)$ \\
$\mathrm{C}(1)-\mathrm{C}(2)-\mathrm{C}(3)-\mathrm{C}(8)$ & $0.44(15)$ \\
$\mathrm{C}(10)-\mathrm{C}(2)-\mathrm{C}(3)-\mathrm{C}(8)$ & $-176.27(14)$ \\
$\mathrm{C}(8)-\mathrm{C}(3)-\mathrm{C}(4)-\mathrm{C}(5)$ & $-1.3(2)$ \\
$\mathrm{C}(2)-\mathrm{C}(3)-\mathrm{C}(4)-\mathrm{C}(5)$ & $178.48(15)$ \\
$\mathrm{C}(3)-\mathrm{C}(4)-\mathrm{C}(5)-\mathrm{C}(6)$ & $0.8(2)$ \\
$\mathrm{C}(4)-\mathrm{C}(5)-\mathrm{C}(6)-\mathrm{C}(7)$ & $0.5(3)$ \\
$\mathrm{C}(5)-\mathrm{C}(6)-\mathrm{C}(7)-\mathrm{C}(8)$ & $-1.1(2)$ \\
$\mathrm{C}(1)-\mathrm{N}(1)-\mathrm{C}(8)-\mathrm{C}(7)$ & $178.62(15)$ \\
$\mathrm{C}(9)-\mathrm{N}(1)-\mathrm{C}(8)-\mathrm{C}(7)$ & $-0.4(2)$ \\
$\mathrm{C}(1)-\mathrm{N}(1)-\mathrm{C}(8)-\mathrm{C}(3)$ & $-0.45(16)$ \\
$\mathrm{C}(9)-\mathrm{N}(1)-\mathrm{C}(8)-\mathrm{C}(3)$ & $-179.48(14)$ \\
$\mathrm{C}(6)-\mathrm{C}(7)-\mathrm{C}(8)-\mathrm{N}(1)$ & $-178.46(15)$ \\
$\mathrm{C}(6)-\mathrm{C}(7)-\mathrm{C}(8)-\mathrm{C}(3)$ & $0.5(2)$ \\
$\mathrm{C}(4)-\mathrm{C}(3)-\mathrm{C}(8)-\mathrm{N}(1)$ & $179.83(12)$ \\
$\mathrm{C}(2)-\mathrm{C}(3)-\mathrm{C}(8)-\mathrm{N}(1)$ & $0.00(15)$ \\
$\mathrm{C}(4)-\mathrm{C}(3)-\mathrm{C}(8)-\mathrm{C}(7)$ & $0.7(2)$ \\
$\mathrm{C}(2)-\mathrm{C}(3)-\mathrm{C}(8)-\mathrm{C}(7)$ & $-179.15(13)$ \\
$\mathrm{C}(1)-\mathrm{C}(2)-\mathrm{C}(10)-\mathrm{C}(11)$ & $-45.1(2)$ \\
$\mathrm{C}(3)-\mathrm{C}(2)-\mathrm{C}(10)-\mathrm{C}(11)$ & $131.10(16)$ \\
$\mathrm{C}(1)-\mathrm{C}(2)-\mathrm{C}(10)-\mathrm{C}(12)$ & $131.83(16)$ \\
& \\
& \\
& \\
& \\
& \\
& \\
&
\end{tabular}




$\begin{array}{lc}\mathrm{C}(3)-\mathrm{C}(2)-\mathrm{C}(10)-\mathrm{C}(12) & -52.0(2) \\ \mathrm{C}(12)-\mathrm{C}(10)-\mathrm{C}(11)-\mathrm{O}(1) & -2.6(2) \\ \mathrm{C}(2)-\mathrm{C}(10)-\mathrm{C}(11)-\mathrm{O}(1) & 174.49(14) \\ \mathrm{C}(11)-\mathrm{C}(10)-\mathrm{C}(12)-\mathrm{O}(2) & -1.6(2) \\ \mathrm{C}(2)-\mathrm{C}(10)-\mathrm{C}(12)-\mathrm{O}(2) & -178.54(14) \\ \mathrm{C}(11)-\mathrm{C}(10)-\mathrm{C}(12)-\mathrm{O}(3) & 179.51(13) \\ \mathrm{C}(2)-\mathrm{C}(10)-\mathrm{C}(12)-\mathrm{O}(3) & 2.5(2) \\ \mathrm{O}(2)-\mathrm{C}(12)-\mathrm{O}(3)-\mathrm{C}(13) & -1.6(2) \\ \mathrm{C}(10)-\mathrm{C}(12)-\mathrm{O}(3)-\mathrm{C}(13) & 177.37(12) \\ \mathrm{C}(12)-\mathrm{O}(3)-\mathrm{C}(13)-\mathrm{C}(15) & 78.08(17) \\ \mathrm{C}(12)-\mathrm{O}(3)-\mathrm{C}(13)-\mathrm{C}(14) & -158.98(14)\end{array}$

Symmetry transformations used to generate equivalent atoms:

Table 7. Hydrogen bonds for PR283 [ $\AA$ and ${ }^{\circ}$ ].

\begin{tabular}{lcccc}
\hline $\mathrm{D}-\mathrm{H} \ldots \mathrm{A}$ & $\mathrm{d}(\mathrm{D}-\mathrm{H})$ & $\mathrm{d}(\mathrm{H} \ldots \mathrm{A})$ & $\mathrm{d}(\mathrm{D} \ldots \mathrm{A})$ & $<(\mathrm{DHA})$ \\
\hline $\mathrm{O}(1)-\mathrm{H}(1 \mathrm{~A}) \ldots \mathrm{O}(2)$ & 0.83 & 1.85 & $2.5628(17)$ & 143.4 \\
\hline
\end{tabular}

Symmetry transformations used to generate equivalent atoms: 